James Madison University JMU Scholarly Commons

Masters Theses

The Graduate School

Summer 2012

\title{
Floristic survey of the vascular plants of Shenandoah County, Virginia
}

Brinton Evan Domangue

James Madison University

Follow this and additional works at: https://commons.lib.jmu.edu/master201019

Part of the Biology Commons

\section{Recommended Citation}

Domangue, Brinton Evan, "Floristic survey of the vascular plants of Shenandoah County, Virginia" (2012). Masters Theses. 189. https://commons.lib.jmu.edu/master201019/189

This Thesis is brought to you for free and open access by the The Graduate School at JMU Scholarly Commons. It has been accepted for inclusion in Masters Theses by an authorized administrator of JMU Scholarly Commons. For more information, please contact dc_admin@jmu.edu. 
Floristic survey of the vascular plants of Shenandoah County, Virginia

\section{Brinton Evan Domangue}

A thesis submitted to the Graduate Faculty of

JAMES MADISON UNIVERSITY

In

Partial Fulfillment of the Requirements

for the degree of

Master of Science

Department of Biology

August 2012 


\section{Acknowledgements}

I would like to thank my advisor Dr. Conley K. McMullen for his mentorship and support throughout the study. He has always been a constant source of encouragement, helping me grow as a person and a scholar. I would also like to thank my committee members, Dr. Heather Griscom and Dr. Michael Renfroe, who have provided me with questions and insight into ways I could improve this project.

I'm thankful to several individuals who have donated their time and resources to help me explore Shenandoah County in ways I could not have done alone. Joseph Lehnen, area forester for the Virginia Department of Forestry, gave me permission and a walking tour of Devil's Backbone State Forest, a biologically diverse area that is otherwise without public access. A few landowners have also provided access to private property and in some cases their own private residence. Thanks to Charles Comer, Dave Evans, Otis Frye, Justin Pence, and Lois Sihler.

I would like to thank Benjamin Burkholder, Jennifer Courtwright and Michelle Lantz for assistance in the field, and to the undergraduate researchers John Bickel, Kathryn Holmes, and Hunter Kiser for help in the James Madison University Herbarium. These individuals devoted many hours to help me through the process. Thanks also to the people who helped coordinate visits to local herbaria: Doug Graber-Neufeld of Eastern Mennonite University, Edgar Lickey of Bridgewater College, and Kelly Ewing of Lord Fairfax Community College.

I'm grateful to Dr. Bruce Wiggins who provided technical support for the use of Geographic Information Systems. I could not have generated the maps in this document without his guidance and expertise. 
For financial support, I greatly appreciate the help of my parents, Rickie and Reinhild Domangue, the Peter T. Nielsen Annual Award for Botanical Studies, and the Cedar Run Church of the Brethren Roy F. Blaine Memorial Scholarship.

Finally, I want to thank everyone who has been by my side along the way. Friends, family, and especially my parents have always motivated and encouraged me, and I would not be where I am today without their love and support. 


\section{Table of Contents}

Acknowledgements ..................................................................................................... ii

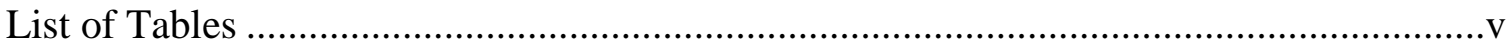

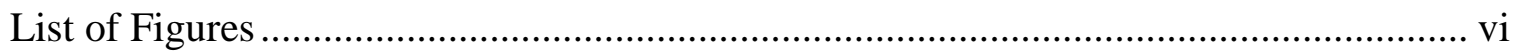

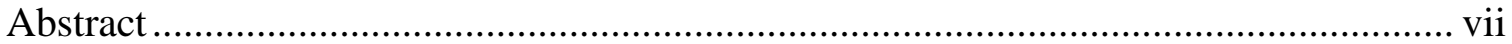

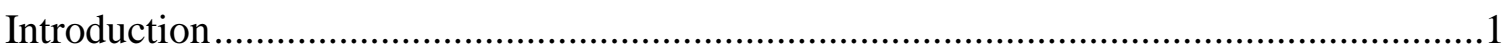

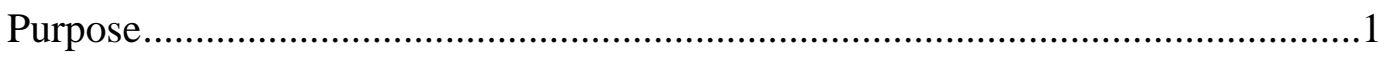

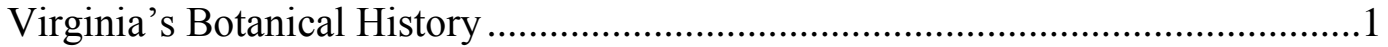

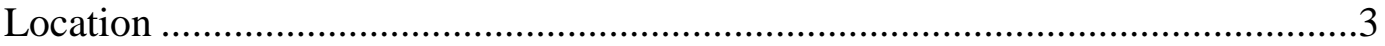

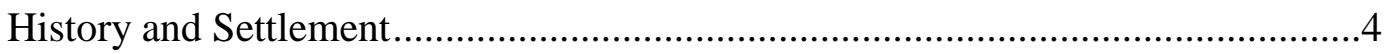

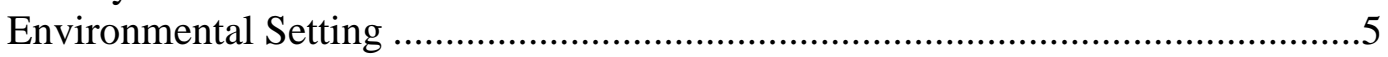

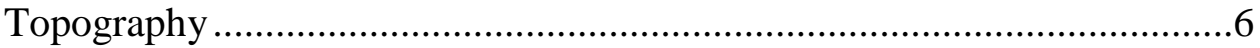

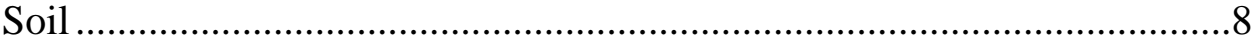

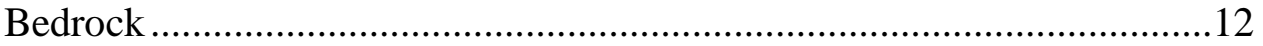

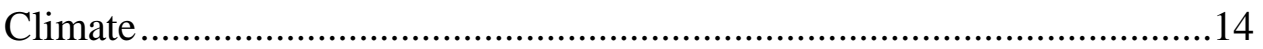

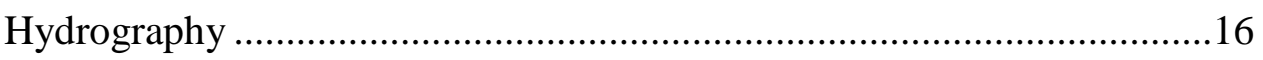

Land Use .....................................................................................18

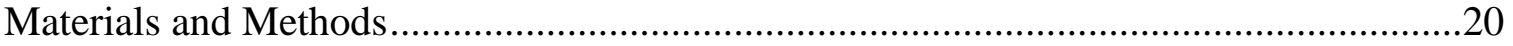

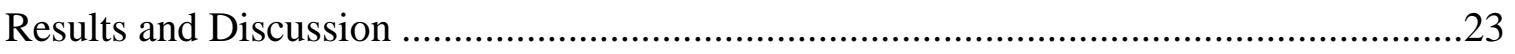

Vegetation Patterns ………………........................................................23

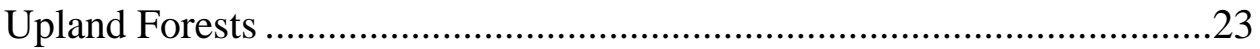

Valley Woodlands..............................................................................24

Open Fields and Fencerows ................................................................25

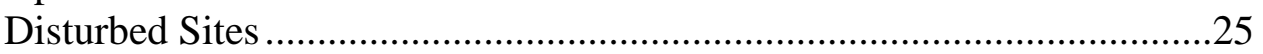

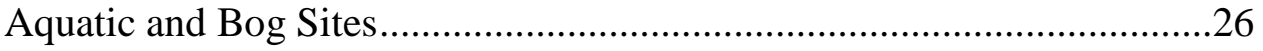

Floodplain and Riparian Zones ..........................................................27

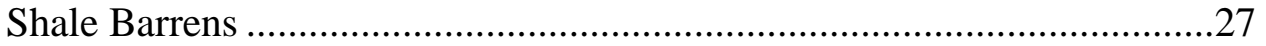

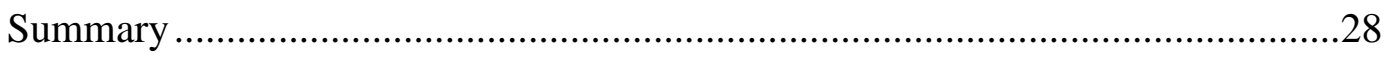

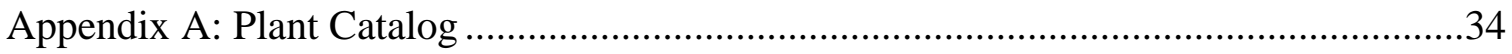

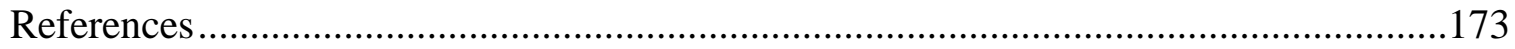




\section{List of Tables}

Table 1. Taxonomic summary of plant families, including resources from new field collections, pre-existing herbarium specimens, and Digital Atlas of the Virginia Flora records. Families are grouped by (a) ferns and fern allies, gymnosperms and monocots,

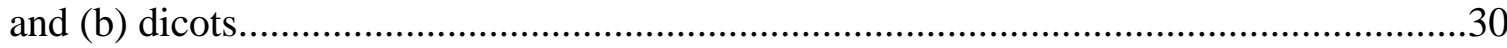




\section{List of Figures}

Figure 1. Location of Shenandoah County, Virginia.....................................................4

Figure 2. Elevation of Shenandoah County, Virginia, with natural ranges from approximately $150 \mathrm{~m}$ to $1,000 \mathrm{~m}$ above sea level.

Figure 3. Soils of Shenandoah County, Virginia, generalized by four major soil orders: Alfisols, Inceptisols, Mollisols and Ultisols. Miscellaneous soils are largely barren land areas that do not support plant growth.

Figure 4. Bedrock underlying Shenandoah County, Virginia, is composed of 15 major formations and groups. These formations are primarily limestone and dolomite in the central part of the county, shale in the eastern central part, and sandstone and shale in the mountains on the eastern and western edges.

Figure 5. Summary climatic data for Shenanandoah County, Virginia for the period of 1981 - 2010, showing average temperatures (a) and average precipitation (b) per month.

Figure 6. Rivers and streams of Shenandoah County, Virginia. The major drainage basin

is the North Fork of the Shenandoah River.

Figure 7. Land use in Shenandoah County, Virginia is comprised of forests (61.6\%), agricultural fields and crops (30.4\%), developed areas (7.3\%) and miscellaneous/open water $(<1.0 \%)$.

Figure 8. Collection sites and topography of Shenandoah County, Virginia. A total of 650 collections were made during the study period. 


\begin{abstract}
The objective of this study was to create a comprehensive record of the vascular plants of Shenandoah County, Virginia. Specimens were collected from the field during the 2011 growing season (March 21 - October 27), and for part of the 2012 growing season (March 22 - May 30). Local herbaria of James Madison University (JMUH), Bridgewater College (BDWR), Eastern Mennonite University (HAVI) and Lord Fairfax Community College (LFCC) were also searched for pre-existing specimens.

The results of the study were compared to records of the Digital Atlas of the Virginia Flora (2012), which currently maps the distribution of vascular plants in Virginia. The survey resulted in 53 new additions to the county record, including 2 new state records for Virginia. The combined results of the field collections, herbarium specimens, and Digital Atlas comprise a total of 1,299 species in 563 genera and 143 families. Of these, 57 species are ferns and fern allies, 8 are gymnosperms, 322 are monocots and 912 are dicots. The records represent 1,014 native species to Virginia, 268 introduced, and 14 unspecified by the Digital Atlas. Voucher specimens of the new field collections are housed in the James Madison University Herbarium.
\end{abstract}




\section{Introduction}

\section{Purpose}

The objective of this study was to compile a comprehensive record of the vascular plants of Shenandoah County, Virginia. Similar projects have been completed in counties throughout the state including adjacent Rockingham County, most recently updated by Peters, McMullen and Hill (2005) in Noteworthy Additions to the Vascular Flora of Rockingham County, Virginia. Shenandoah County was selected in an effort to contribute to the knowledge of the Virginia flora.

According to records from the Virginia Botanical Associates' Digital Atlas of the Virginia Flora (2012), there were already between 1,240 and 1,250 vascular plants known to occur in Shenandoah County. The purpose of the current survey was to supply new data from field collections and from existing specimens found in local herbaria. Voucher specimens from new field collections are housed in the James Madison University Herbarium (JMUH).

Aside from its primary purpose, the results of this project may benefit other fields of study. Each collection includes a general description of the specimen and the collection site, providing information useful to conservationists, ecologists, and anyone interested in the local environment. Additionally, specimens in the herbarium provide access to a large repository of plant material, making pollen, seeds, and even DNA available for research.

\section{Virginia's Botanical History}

Beginning in the mid- $16^{\text {th }}$ century, Virginia was at the heart of botanical discovery and exploration. Floristic endeavors progressed slowly at first with some early 
collections made by Thomas Harriot. He took approximately 30 species back to England after an expedition to the Americas, though not all of these collections were from Virginia. Between 1632 and 1654, a collector by the name of John Tradescant made trips specifically to Virginia, collecting mostly along the York River. Tradescant took back approximately 100 species to England. In 1678, even greater contributions were made by John Banister who collected in Virginia for 14 years and sent plant material, specimens and drawings to Oxford (Harvill et al. 1992).

Interest in the New World flora gained momentum in the $18^{\text {th }}$ century. A wellknown botanist of Virginia, John Clayton, began sending specimens to English naturalists in the 1720s and 1730s. These records were received by botanists John Gronovius and Carl Linnaeus. Gronovius used Clayton's records to publish Flora Virginica in 1739, the first published Flora of the new world (Purvis 1999). One of Clayton's more notable contemporaries was John Bartram who collected throughout the eastern United States, including Virginia. Bartram made connections with the scientific community of the Old World, sending plant material and live specimens overseas for nearly 40 years (Hoffmann and Horne 2004).

Botanical work slowed during the $19^{\text {th }}$ century, and proposed Virginia flora projects fell through on a few occasions. One of these projects was an effort by Dr. Benjamin Smith Barton to update Clayton's Flora Virginica, but the work was never fully completed. Other notable botanists of this period were Nathaniel Britton, John Small, John Lyon, Frederick Pursh, and Asa Gray. Most of the collections made at this time were few and far between, but Pursh collected some Virginia plants and included them in Flora Americae Septentrionalis, published in 1814 (Harvill et al. 1992). Asa 
Gray's noteworthy publication of 1848 was Manual of the Botany of the Northern United States, which in 1856 was revised in a second edition to include all of Virginia and Kentucky (McMullen 1980).

In the $20^{\text {th }}$ century, botanical research picked up again and floristic studies became more thorough, often focusing on localized areas. For instance, Earl J. Grimes along with his wife studied the entire Virginia Peninsula, which culminated in 1924 with the publication of Flora of the Peninsula of Virginia. A number of other botanists also specialized in areas of certain habitats or regions. Contributions came from H. A. Allard who focused on Bull Run Mountain and areas of northern Virginia. Lena Artz studied flora of the Massanutten Mountains. Lloyd Carr made collections around the ponds and wetlands of Augusta County. Then in 1961, A. B. Massey published Virginia Flora. This was the first catalog since Clayton's Flora Virginica that accounted for Virginia's flora as a whole (Harvill et al. 1992).

The most recent and complete reference to Virginia flora, however, is the Atlas of Virginia Flora, by Alton Harvill, et al. There are three printed editions of this manual. The first edition was published in two parts in 1977 and 1981, the second edition was published in 1986, and the third in 1992. A fourth edition is available online as the Digital Atlas of the Virginia Flora (Virginia Botanical Associates 2012). This resource is frequently updated and provides distribution records for known Virginia ferns and fern allies, gymnosperms, monocots, and dicots.

\section{Location}

Shenandoah County is part of the Shenandoah Valley on the northwestern edge of Virginia, where it borders Hardy County of West Virginia (Figure 1). It is bordered by 
Frederick County to the north, Warren and Page Counties to the east, and Rockingham County to the south. Generally elongated in a northeast to southwest direction, the county is approximately $58 \mathrm{~km}$ long and averages between 24 and $27 \mathrm{~km}$ wide. The total land area is roughly 1,320 square kilometers (Crater PDC 2010). Major geographical features are the Massanutten Mountain range, which lies on the eastern edge of the county and the Great North Mountain range on the western border. Through the valley runs the North Fork of the Shenandoah River.

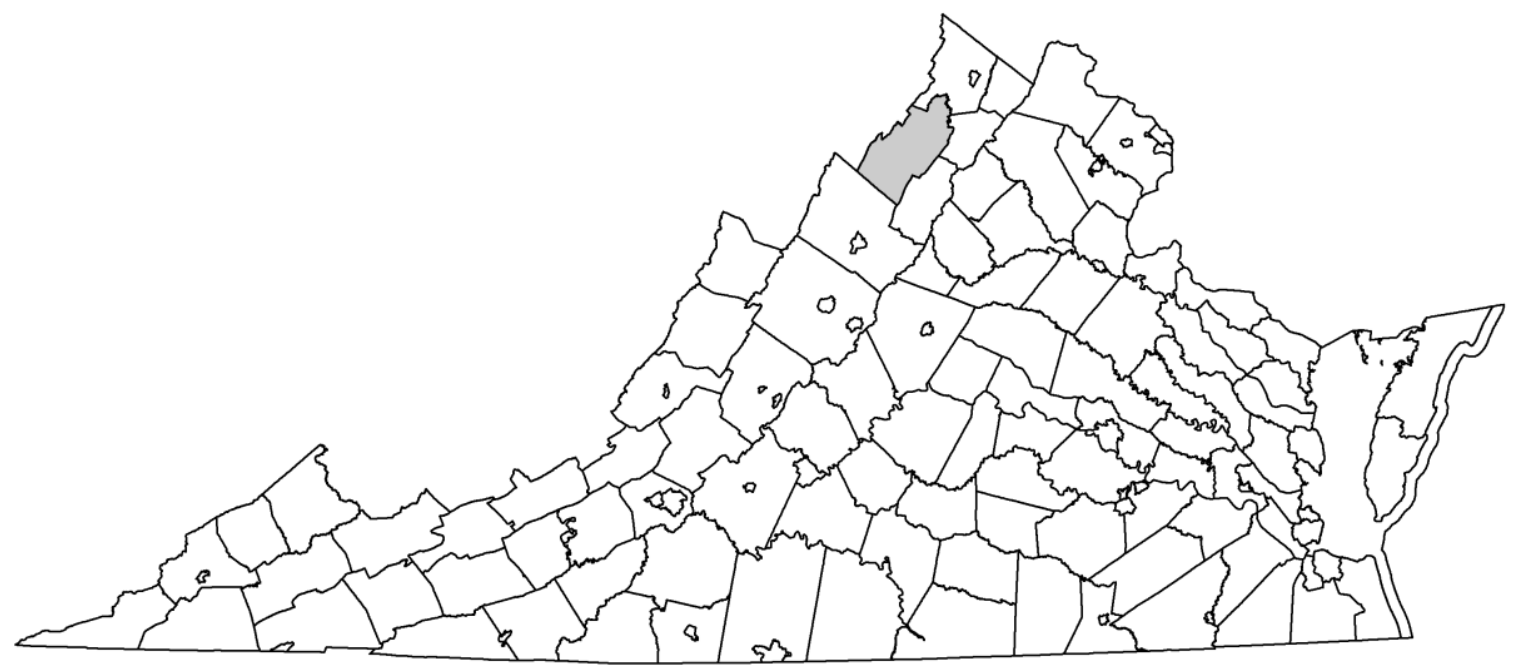

Figure 1. Location of Shenandoah County, Virginia.

\section{History and Settlement}

In the late $17^{\text {th }}$ and early $18^{\text {th }}$ centuries, explorers were making their way into the area that would become Shenandoah County. The English government at the time encouraged expansion in an effort to colonize new land and to investigate Native American establishments (Wayland 1980). Native populations are believed to have had a significant impact on the ecosystem. There is evidence of frequent burning throughout the valley and reports of widespread grasslands. The function of this practice may have 
been for agricultural or hunting purposes (Brown 2000). When the native populations were pushed westward in the mid-1700s, much of the land returned to forest (Wayland 1980).

White settlements appeared as early as 1727 in the Massanutten area, and by 1731 - 1734, were also known from the Shenandoah side of Cedar Creek and the land near Mount Jackson (Wayland 1980). Settlers were mostly of Scottish, Irish, and German decent and travelled into the county from Pennsylvania (Shenandoah County Citizens' Advisory Committee 2005). Towns soon began forming along the North Fork of the Shenandoah River and along what is now Route 11. Many of these communities have become the major incorporated towns of today: Strasburg, Toms Brook, Woodstock, Edinburg, Mount Jackson and New Market.

The area that would become Shenandoah County was originally part of Frederick County until 1772 when Shenandoah was officially established. The original name was Dunmore County but was renamed to Shenandoah in 1778. Until 1831, Shenandoah included parts of Warren County to the east and parts of Page County to the southeast. It was at this time that Page County was formed from Shenandoah and Rockingham County. Then, in 1836, Warren County was formed from Shenandoah and Frederick County, leaving Shenandoah to its present-day shape (Wayland 1980).

\section{Environmental Setting}

The diverse landscape and climate of Shenandoah County has a substantial impact on the local flora. Researchers attempting to analyze the influence of environmental factors on species diversity commonly focus on one or two features at a time; however, species richness is often better understood from a multi-factor approach (Pausas and 
Austin 2001). Hence, it is relevant to report and understand the physical attributes of the county. Reviewers on the topic have found that environmental factors such as topography, climate, soil and geology can influence species diversity (Pausus and Austin 2001; Lundholm 2009).

\section{Topography}

Shenandoah County is part of the Ridge and Valley province of Virginia. This physiographic province includes the rolling hills of the valley and the mountains of the surrounding area. The county is bordered on the east by the Massanutten Mountain Range and on the West by the Appalachians (USDA, Soil Conservation Service 1991).

The three main components of topography are elevation, slope and aspect. Of the three, elevation is considered the most influential on vegetation patterns (Young et al. 2009; Miller 1986). The lowest elevations are around $150 \mathrm{~m}$ above sea level and occur in the north-western part of the county along the North Fork of the Shenandoah River. However, pits of the nearby Strasburg Quarry may reach several meters below this level. The highest elevation is 1,003 $\mathrm{m}$ at Great North Mountain on the western edge of the county (Fig. 2; USGS 2009). 


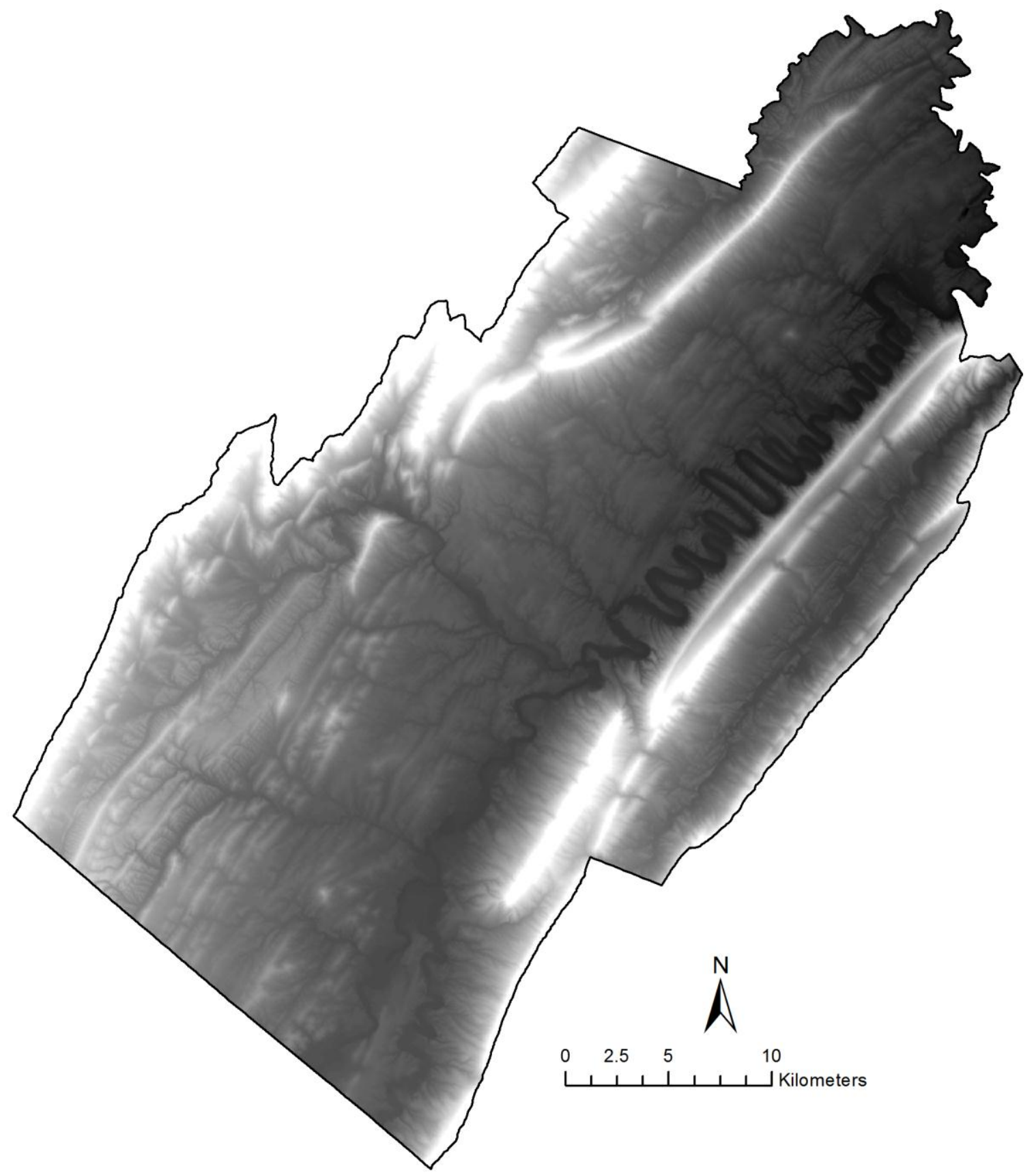

Figure 2. Elevation of Shenandoah County, Virginia, with natural ranges from approximately $150 \mathrm{~m}$ (dark) to $1,000 \mathrm{~m}$ (light) above sea level. 


\section{Soil}

Soils in Shenandoah County are classified into series within four main soil orders. These groups make up the Alfisols, Inceptisols, Mollisols, and Ultisols (Fig. 3; USDA, Natural Resources Conservation Service 2008).

Most Alfisols have an ochric epipedon and an argillic horizon containing clay leached from the upper surface. These soils are typically formed in forest or savanna, and have a medium to high saturation of bases. Water is generally available in the soil for vegetative growth. These combined properties make them suitable for cultivation (Soil Survey Staff 1999). Alfisols in Shenandoah County are mainly located in the central valley area and make up nearly $13 \%$ of the soils. Some major series that make up this group are the Endcav, Chilhowie and Carbo-Endcav complex, all formed from calcareous shale and limestone. These three groups are generally silt loam to silty clay loam on slopes from $2-55 \%$ and can be rocky to very rocky. Other series belonging to the Alfisols in Shenandoah County include Carbo, Caverns, Edom, Maurertown, Opequon and Toms (USDA, Natural Resources Conservation Service 2008).

Inceptisols encompass a wide range of characteristics and environments, but the most common types have an ochric or umbric epipedon and a cambric horizon. These soils are considered less developed than other soil orders from a lack of weathering; they occur on mountain slopes around newly exposed surfaces and in river valleys from recently deposited sediments (Soil Survey Staff 1999). In the county, Inceptisols make up approximately $28 \%$ of the soils. Major series are the Wallen, Weikert-Berks complex and Berks-Weikert complex. The Wallen series is a channery sandy loam weathered from sandstone. The series is present on slopes from $2-70 \%$. The Weikert-Berks and Berks- 
Weikert complexes are channery silt loam soils formed from sandstone and shale on slopes from $7-70 \%$. These three groups can be very to extremely stony. Minor series include Berks, Derroc, Weikert, and the Wallen-Lehew complex (USDA, Natural Resources Conservation Service 2008).

Mollisols have a mollic epipedon, characterized by a thick, dark upper layer, and typically an argillic, natric or calcic horizon. These soils are rich in bases. Most were formed under grasses but are known from forest areas, as well (Soil Survey Staff 1999). Mollisols collectively only make up about $2 \%$ of the soils in Shenandoah County. Series include Broadway, Gladehill, Massanetta, Newmarc, Nomberville, and Wolfgap. The majority of these are loam or silty loam, on slopes of $0-5 \%$. Located on floodplains in the valley, these soils are composed of alluvium from limestone, sandstone and shale materials (USDA, Natural Resources Conservation Service 2008).

Ultisols are similar to Alfisols with an accumulation of clay in the lower horizons and an adequate supply of moisture. Ultisols, though, leach a large amount of other materials and are relatively low in bases. They usually have an ochric epipedon and an argillic or kandic horizon. These soils were formed in forested areas (Soil Survey Staff 1999). Ultisols are the most common group of soils in the county at around $29 \%$. Representatives of this group include the Laidig, Gilpin and Unison series. Located on hills and uplands, Laidig is a channery loam formed from sandstone and shale. Gilpin may be a silt loam or a channery silt loam formed from shale, sandstone and siltstone. Both Laidig and Gilpin can be very stony on slopes from $2-55 \%$. Unison is a loam that may be gravelly or cobbly on slopes of $2-15 \%$. These soils are found on stream terraces within the valley and are composed of alluvium from acid crystalline rocks. Additional 
series are found to a lesser extent throughout the county: Alonzville, Blairton, Braddock, Coursey, Frederick, Guyan, Jefferson, Massanutten, Moomaw, Purdy, Sequoia, Timberville, Trappist, Zepp (USDA, Natural Resources Conservation Service 2008). Other series may form complexes that cross taxonomic orders (Fig. 3). Lehew and Gainesboro soils together are about $2 \%$ of the county and make up a group of InceptisolsUltisols. The soils are derived from sandstone and shale and are located mostly in the uplands of the southwestern part of the county. The Frederick and Poplimento silt loams are found on hills and uplands in the central part of the valley and make up a group of Ultisols-Alfisols. The Frederick component of this group is derived from mostly dolomitic limestone, while the Poplimento component is derived from limestone, shale and siltstone. These soils together are around $19 \%$ of the soils in the county. The Gainesboro-Berks complex makes up a group of Ultisols-Inceptisols and is less than $1 \%$ of the total soils. Small plots located on uplands near the western edge of the county are derived from weathered shale, sandstone and siltstone (USDA, Natural Resources Conservation Service 2008).

Areas that are generally unsuitable for vegetative growth are designated as "Miscellaneous" (Fig. 3). These include mostly rock outcrops on mountain ridges, but can also be pits, dumps, and urban development (USDA, Natural Resources Conservation Service 2008). 


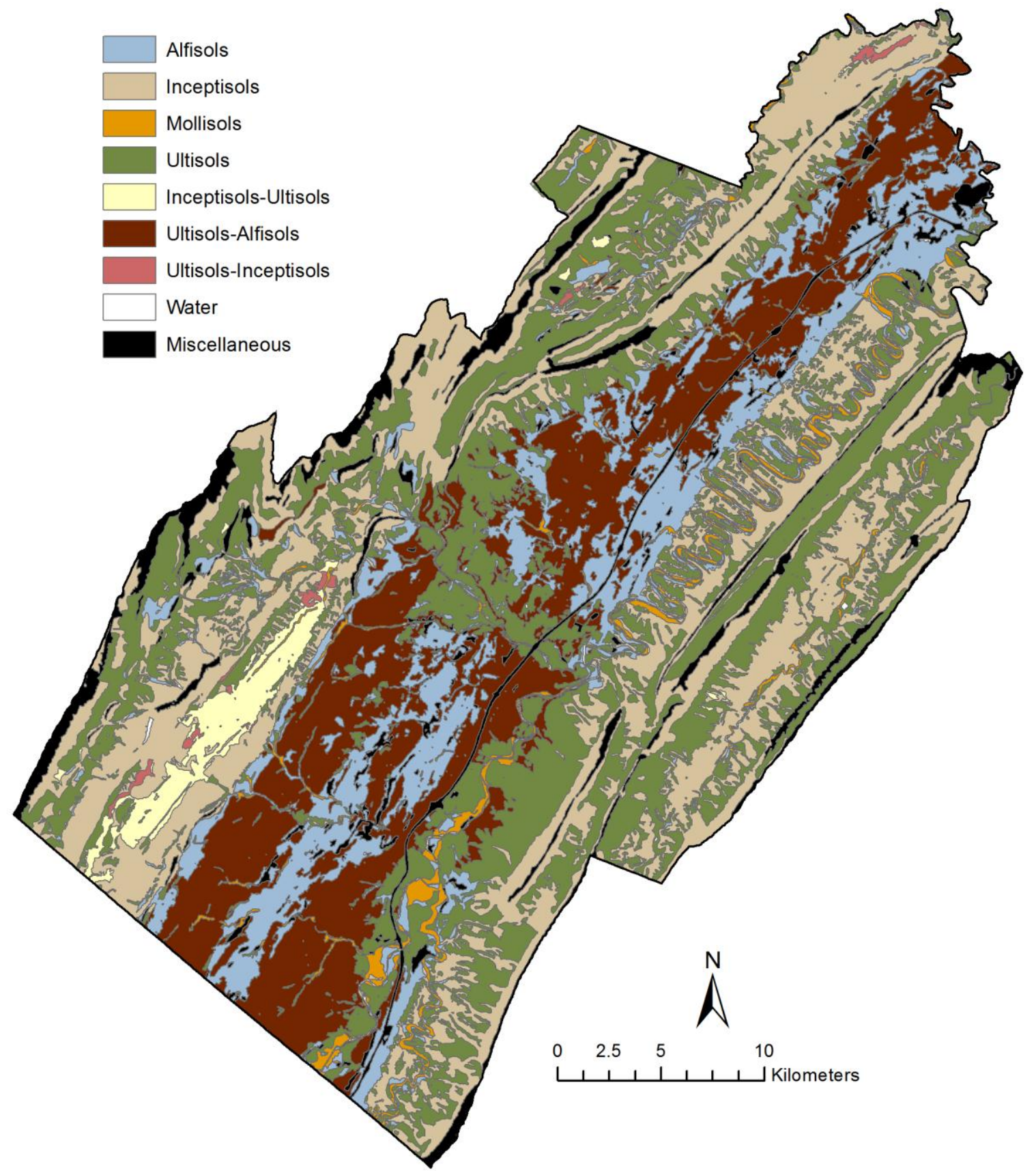

Figure 3. Soils of Shenandoah County, Virginia, generalized by four major soil orders: Alfisols, Inceptisols, Mollisols and Ultisols. Miscellaneous soils are largely barren land areas that do not support plant growth. 


\section{Bedrock}

The underlying bedrock in Shenandoah County is of the Paleozoic era and covers periods from Cambrian, Ordovician, Silurian, and Devonian age. Along the center of the valley between the bordering mountain ranges are units of Cambrian and Ordovician age. Of this period, the bedrock is primarily limestone and dolomite west of U.S. Highway 11. The formations here include the Beekmantown Group; Conococheague Formation; Edinburg Formation, Lincolnshire and New Market Limestones; and Elbrook Formation. To the east of this marker, the bedrock is primarily shale. The Martinsburg and Oranda formations belong to this area. The Juniata, Oswego, Martinsburg (Reedsville and Dolly Ridge), and Eggleston Formations which are also mostly shale are found to the northwest of the county (Fig. 4; Dicken et al. 2005).

The mountain ranges on either side of the county are made up of Silurian and Devonian units. This bedrock is composed primarily of sandstone and shale. The formations in this section include the Brallier Formation; Chemung Formation; Hampshire Formation; Keefer, Rose Hill, and Tuscarora Formations; Mahantango Formation; Marcellus Shale and Needmore Formation; Massanutten Sandstone; Millboro Shale and Needmore Formation; and the Ridgeley Sandstone, Helderberg and Cayugan Groups (Fig. 4; Dicken et al. 2005). 
Beekmantown

Brallier

Chemung

Conococheague

Edinburg, Lincolnshire \& New Market Limestones

Elbrook

Hampshire

Juniata, Oswego, Martinsburg, \& Eggleston

Keefer, Rose Hill, \& Tuscarora

Mahantango

Marcellus Shale \& Needmore

Martinsburg \& Oranda

Massanutten Sandstone

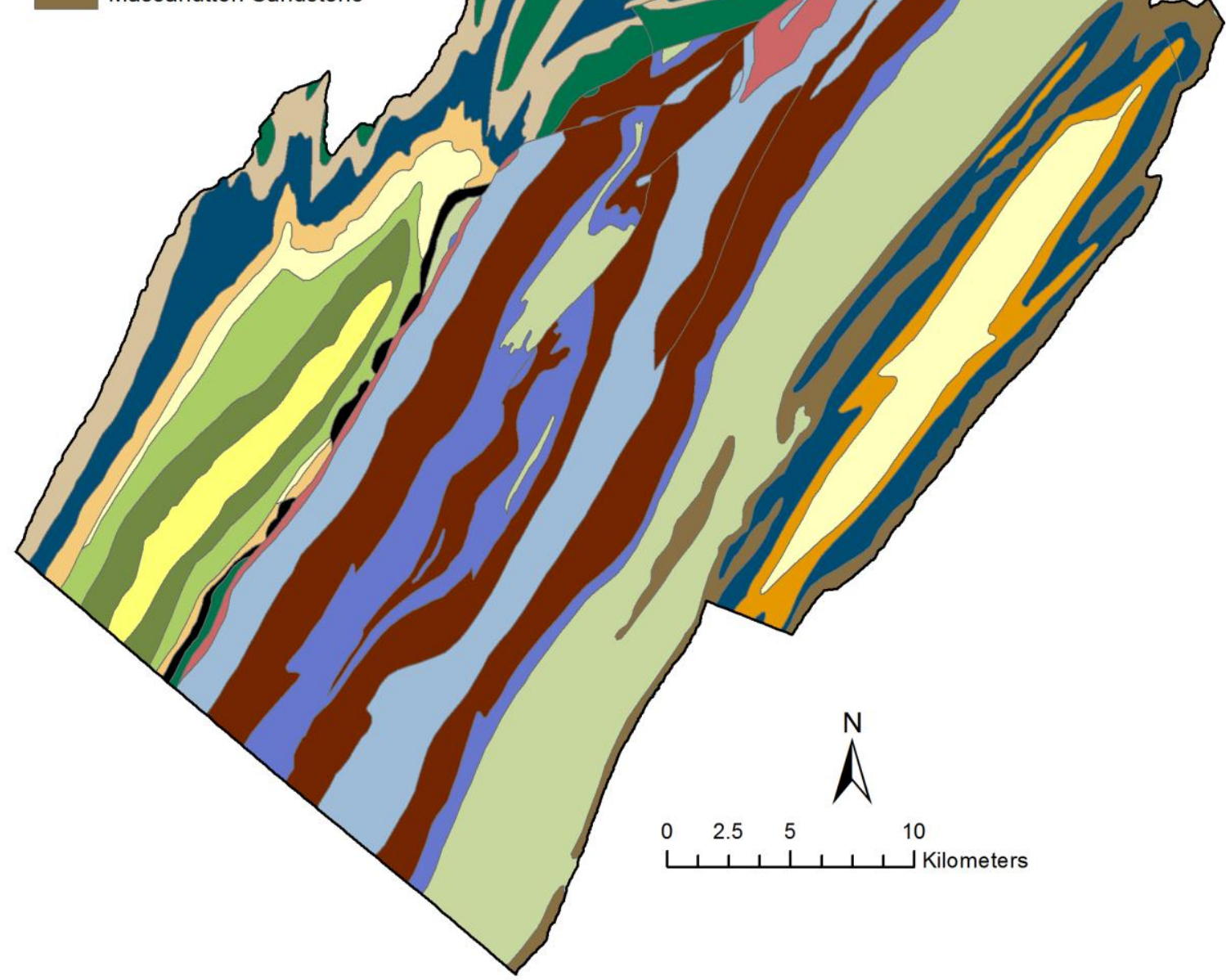

Figure 4. Bedrock underlying Shenandoah County, Virginia, is composed of 15 major formations and groups. These formations are primarily limestone and dolomite in the central part of the county, shale in the eastern central part, and sandstone and shale in the mountains on the eastern and western edges. 


\section{Climate}

The climate in Shenandoah County is classified as warm temperate and fully humid with hot summers (Rubel and Kottek 2010). From data collected at the Woodstock station (Fig. 5), the mean annual temperature is $11.1^{\circ} \mathrm{C}$. The lowest temperatures occur in January with an average of $-0.8{ }^{\circ} \mathrm{C}$, and reach a peak in July with an average of 23.1 ${ }^{\circ} \mathrm{C}$. Precipitation for the area averages $97.2 \mathrm{~cm}$ per year with snow accumulation averaging $66.3 \mathrm{~cm}$ per year. A summary of monthly climate data for the period of 1981 to 2010 is shown in Table 1 (USDC, National Climatic Data Center 2010).

On a smaller scale, elevation may have an impact on climate within the county. In the valley, average annual temperatures can be $1-2{ }^{\circ} \mathrm{C}$ warmer than higher elevations of the surrounding mountains, with a total range of approximately 8 to $12{ }^{\circ} \mathrm{C}$ (The PRISM Climate Group 2006a,b). 
a) _ Daily Max. Daily Min. _ Average

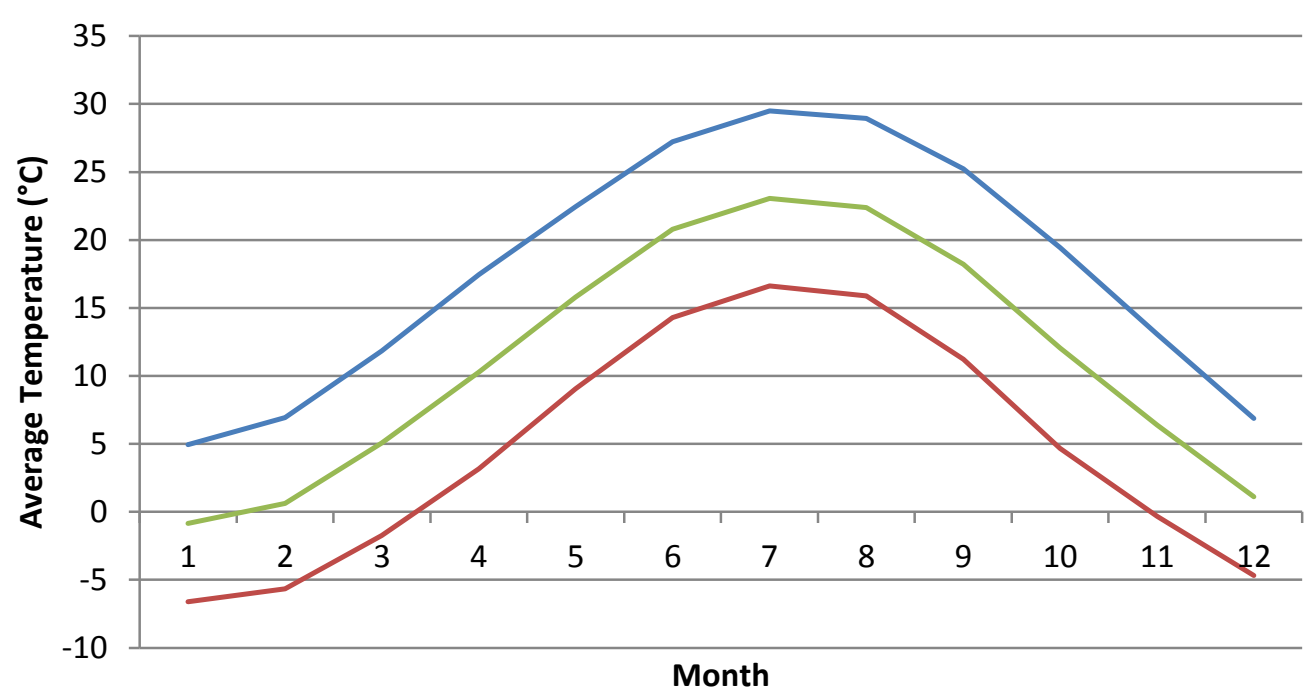

b) $\quad$ Avg. Precipitation - Avg. Snow

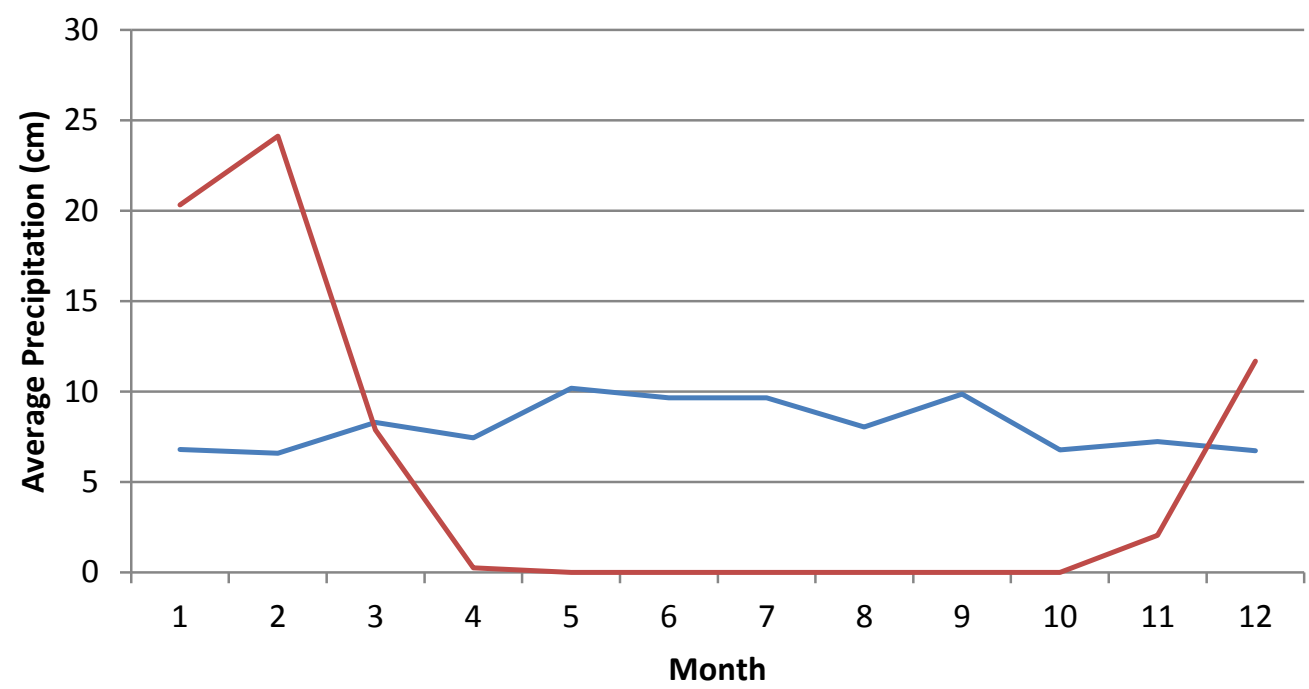

Figure 5. Summary climatic data for Shenanandoah County, Virginia for the period of $1981-2010$, showing average temperatures (a) and average precipitation (b) per month. 


\section{Hydrography}

The major drainage basin in Shenandoah County is the North Fork of the Shenandoah River. Eventually all water runoff that originates in the county flows into the North Fork (Shenandoah County Citizens' Advisory Committee 2005), which then meets the South Fork at Front Royal in Warren County. Further downstream, the Shenandoah enters the Potomac and then the Chesapeake Bay. Holmans Creek, Mill Creek and Smith Creek drain the southern part of the county while Pughs Run, Stony Creek and Narrow Passage Creek drain the central part. To the north, the area is drained by Toms Brook, Tumbling Run and Cedar Creek, the latter of which also forms the northern boundary of the county. Fort Valley, which lies between the twin ridges of the Massanutten Mountains, is drained by Passage Creek. This waterway merges into the North Fork a few kilometers outside of the county to the northeast (Fig. 6).

Approximately $72 \%$ of the annual precipitation returns to the atmosphere by evapotranspiration while 17\% remains in the rivers and streams as surface water. Another $11 \%$ becomes groundwater. Groundwater readily penetrates much of the central part of the county, which is made up of karst landforms. Karst is formed by the dissolution of carbonate bedrock resulting in pores, cracks, sinkholes and caverns (Shenandoah County Citizens' Advisory Committee 2005).

Groundwater is an important resource in the region. It often returns to the surface naturally by springs and interconnected channels, contributing on average $30 \%$ to the flow of waterways aboveground. Groundwater also returns to the surface by wells. Approximately $80 \%$ of the county's population relies on groundwater for daily use, and around areas of karst topography, aquifers supply enough for agricultural, domestic and 
public uses. In the uplands where bedrock is mostly sandstone and shale, groundwater is less abundant and generally provides only enough for rural and domestic use (WRAC n.d.).

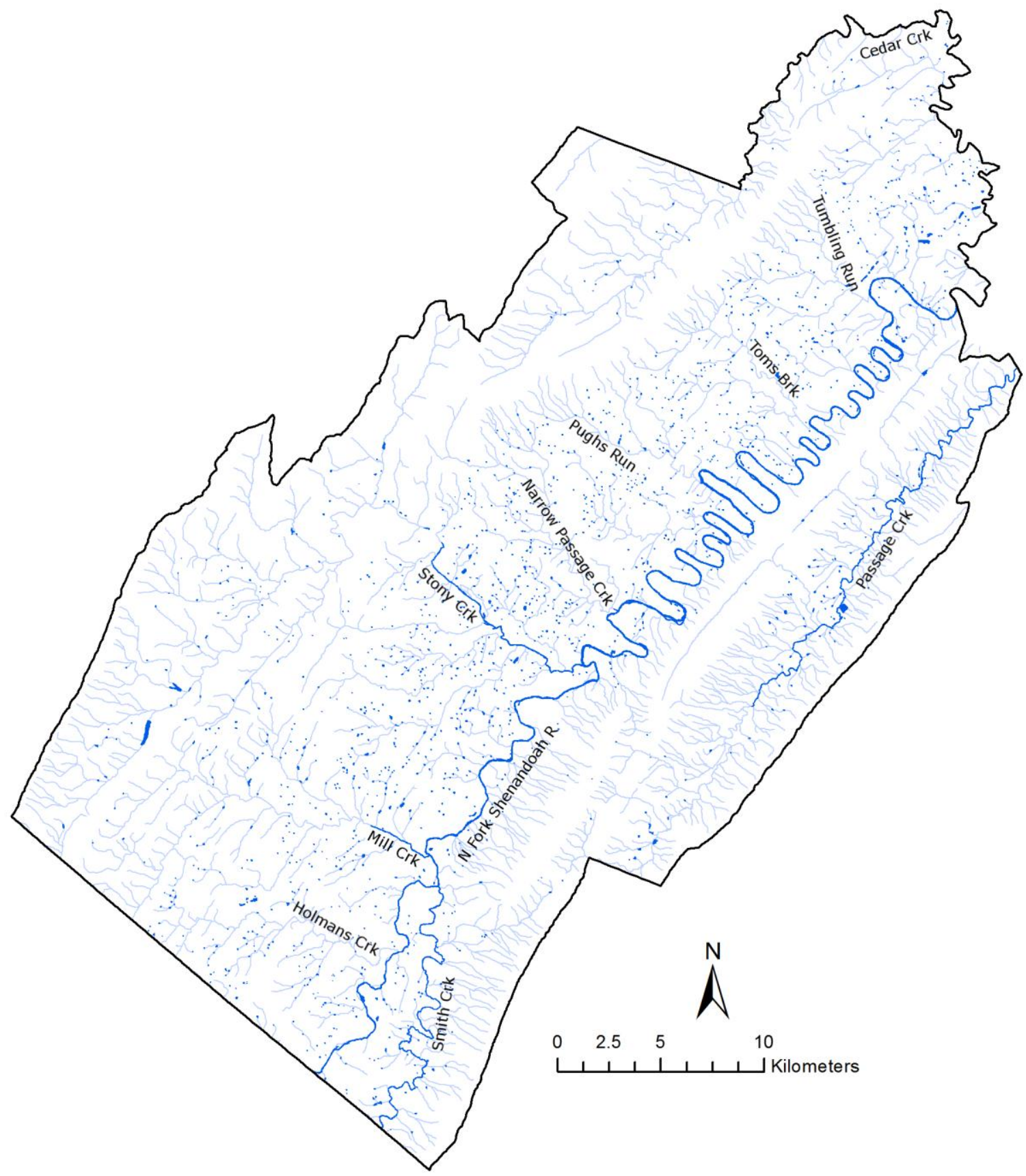

Figure 6. Rivers and streams of Shenandoah County, Virginia (USDC, U.S. Census Bureau, Geography Division 2010a,b). The major drainage basin is the North Fork of the Shenandoah River. 


\section{Land Use}

Shenandoah County is primarily rural. National land cover data from the U.S. Geological Survey (Fig. 7; 2006) indicates that about $30.4 \%$ of the total land area is used for agriculture. However, according to the 2007 Census of Agriculture, $572 \mathrm{~km}^{2}$ are "land in farms" which is close to $43 \%$ of the total land area, but this statistic also includes 124 $\mathrm{km}^{2}$ of woodland. Of the total farmland, $244 \mathrm{~km}^{2}$ are invested in crops. Forage crops are the most common, followed by corn and soybeans. A large part of the remaining farmland, $182 \mathrm{~km}^{2}$, is permanent pasture and rangeland with $21 \mathrm{~km}^{2}$ described as farmsteads, buildings, facilities, etc. (USDA, National Agricultural Statistics Service 2009).

Forested land comprises about $61.6 \%$ of the total area. These areas are broken down into deciduous forest (52.1\%), evergreen forest (6.0\%), and mixed forest $(3.5 \%)$. Of the total forested land area, $36.6 \%$ is federally owned as part of the George Washington and Jefferson National Forest. At the state level, $0.2 \%$ belongs to Devil's Backbone State Forest in the north-western part of the county. The rest of the wooded land area is privately owned (USGS 2011; VA-DCR 2012a,b).

Developed areas represent $7.3 \%$ of the county. The amount of vegetation in these areas depends on the level of development. Residential areas can be mostly vegetation in the form of lawns and parks, whereas highly developed areas in populated towns will have mostly impervious surfaces with little vegetation. This land use category includes much of the transportation network as well (USGS 2011).

The remaining land area makes up less than $1 \%$ of the county. Most are areas unsuitable for vegetative growth such as barren land and open water (USGS 2011). 


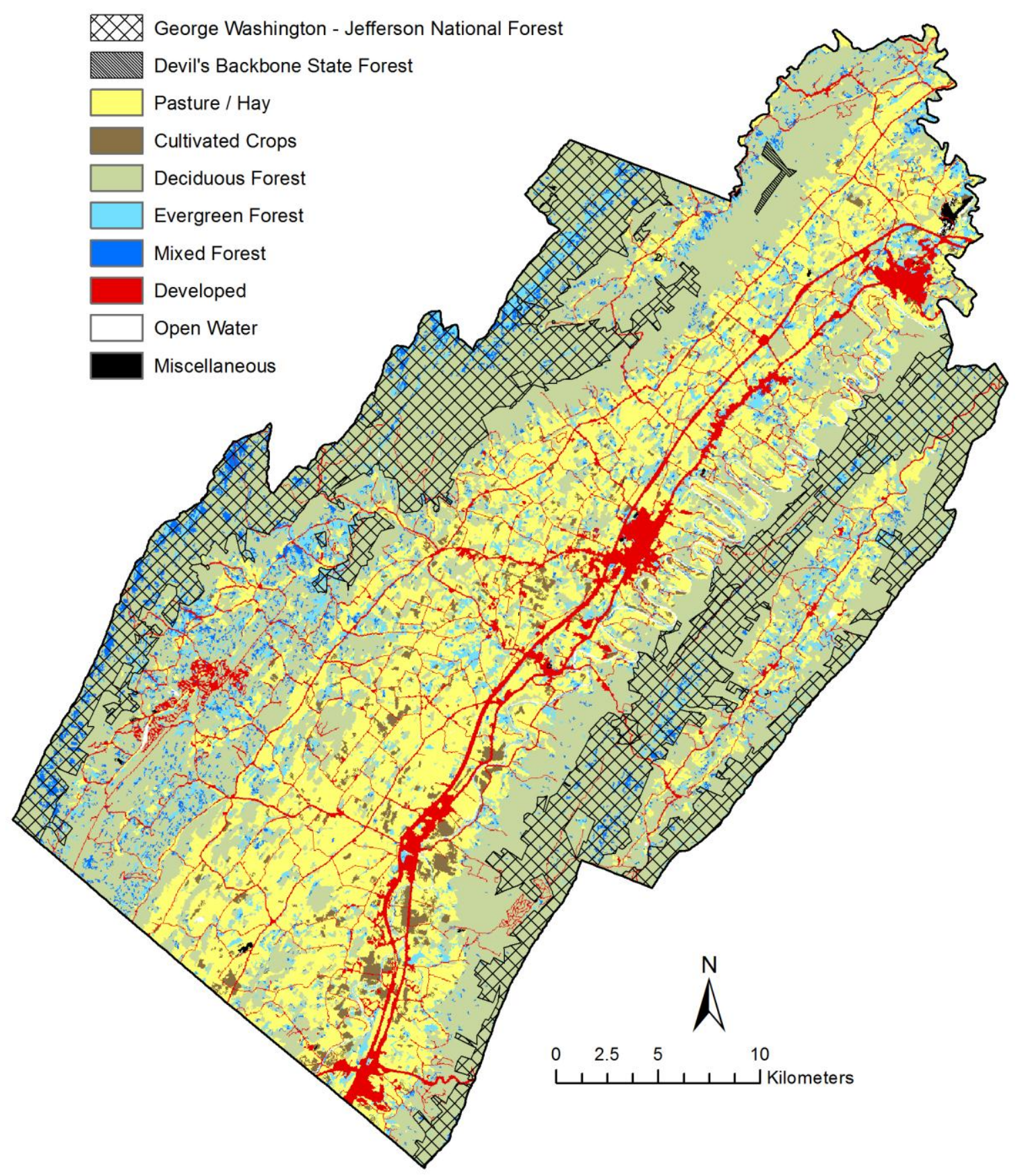

Figure 7. Land use in Shenandoah County, Virginia is comprised of forests $(61.6 \%)$, agricultural fields and crops $(30.4 \%)$, developed areas $(7.3 \%)$ and miscellaneous/open water $(<1.0 \%)$. 


\section{Materials and Methods}

Vascular plant specimens were collected within Shenandoah County during the 2011 growing season (March 21 - October 27) and part of the 2012 growing season (March 22 - May 30). During these periods, field trips were taken, on average, once a week. Sites were revisited as needed to account for phenological diversity.

Collection sites were based largely on accessibility. The least restricted areas are national forest, public lands, and transportation/utilities. Combined, these areas are only a quarter of the total unincorporated land area (Shenandoah County Citizens' Advisory Committee 2005), so permissions were granted to survey Devil's Backbone State Forest and other private properties throughout the county. Transportation was also an important factor determining accessibility. The road network is extensive along the center of the valley but there is considerably less access to slopes of the mountain ridges. Most collection sites were chosen due to their proximity to roads.

Basic shearing and digging tools were used to collect plant material. Specimens from the field were collected only when reproductive structures such as spores, flowers, fruit, seeds and cones were present. These features were necessary for accurate identification and the creation of useful herbarium specimens. Information about each collection was recorded in a field notebook and included the collection date, location,

number, and species name. Precise locations were recorded by GPS coordinates using a Garmin GPSmap 76CSx. Additional information that was sometimes included were physical plant features that would not be obvious from a dried specimen, ecological setting, and associated species if applicable. Once collected, plants were pressed, dried 
and mounted using standard herbarium techniques. The resulting herbarium specimens are stored in the James Madison University Herbarium (JMUH).

Plants were identified in the field or from material brought back to the herbarium. Flora of West Virginia (Strausbaugh and Core 1978), The Plants of Pennsylvania: An Illustrated Manual (Rhoads and Block 2000), Manual of Vascular Plants of Northeastern United States and Adjacent Canada (Gleason and Cronquist 1991) and Newcomb's Wildflower Guide (Newcomb 1977) were used as references for identification. Scientific nomenclature was kept consistent with the Virginia Botanical Associates' Digital Atlas of the Virginia Flora (2012) and was often compared to the U.S. Department of Agriculture (USDA) PLANTS Database (2012) to resolve differences in taxonomy. The Digital Atlas was also used to determine native status, while common names followed that of the PLANTS Database. Species authorship was in some cases further abbreviated for consistency.

In addition to the field study, local herbaria were searched for pre-existing county specimens. The four herbaria nearest to Shenandoah County are James Madison University (JMUH), Bridgewater College (BDWR), Eastern Mennonite University (HAVI) and Lord Fairfax Community College (LFCC).

BDWR and HAVI both contained less than 5,000 specimens each. Records from BDWR were received in electronic format so this herbarium was not visited. JMUH and LFCC contained collections of nearly 20,000 specimens each. Although none of these herbaria are located within the county, LFCC is the closest, lying only a few kilometers northeast of the border. As a result, a considerably larger number of Shenandoah specimens were supplied from this herbarium. To simplify these findings, the LFCC 
records were abridged for this survey; only the earliest and most recent specimens of a single species were reported. The identities of specimens representing new county records from all herbaria were verified before inclusion in the survey 


\section{Results and Discussion}

\section{Vegetation Patterns}

Shenandoah County's environmental heterogeneity generates a diverse set of flora, and plant communities take form within several habitat types. The generalized habitats are upland forests, valley woodlands, open fields and fencerows, aquatic and bog sites, floodplain and riparian zones, disturbed sites, and shale barrens.

\section{Upland Forests}

Upland forests are dominated by oak-hickory associations. Common oak species include Quercus alba, Q. marilandica, Q. montana, Q. rubra, and Q. stellata, and common hickory species are Carya glabra and $C$. ovata. In mixed forests, other canopy trees include Pinus pungens, $P$. rigida, and $P$. virginiana. Among smaller trees and woody shrubs of the understory are Acer pensylvanicum, Amelanchier arborea, Betula lenta, Cornus florida, Gaylussacia baccata, Kalmia latifolia, Menziesia pilosa, Rhododendron maximum, $R$. periclymenoides, Vaccinium angustifolium, V. pallidum, $V$. stamineum, and Viburnum acerifolium.

Herbaceous plants include several species of ferns and fern allies, including Dennstaedtia punctilobula, Dryopteris marginalis, Lycopodium clavatum, Polypodium virginianum, Polystichum acrostichoides, and Thelypteris noveboracensis. Additional herbaceous plants are Epigaea repens, Eurybia divaricata, Hieracium venosum, Iris verna, Micranthes virginiensis, Monotropa uniflora, Packera obovata, Parthenium integrifolium, Polygonatum biflorum, and Pycnanthemum tenuifolium. 


\section{Valley Woodlands}

Vegetative composition of valley woodlands usually overlaps with upland forests, and contains many of the same oak-hickory associations. Other tree species such as Acer rubrum, A. saccharum, Castanea pumila, Fraxinus americana, Liriodendron tulipifera, Prunus serotina, and Robinia pseudoacacia all commonly occur in valley woodlands. Smaller woody plants include Cercis canadensis, Hamamelis virginiana, Lindera benzoin, Ostrya virginiana, Physocarpus opulifolius, Ptelea trifoliata, Sassafras albidum, Viburnum dentatum, and V. prunifolium.

Pteridophytes of upland forests also occur at lower elevations. Additional ferns and fern allies not mentioned previously include Asplenium platyneuron, Botrychium virginianum and Diphasiastrum digitatum. Many other herbaceous plants frequent these woods: Anemone virginiana, Antennaria plantaginifolia, Aquilegia canadensis, Asclepias quadrifolia, Cardamine concatenata, Ceanothus americanus, Chimaphila maculata, Chrysogonum virginianum, Cimicifuga racemosa, Claytonia virginica, Corydalis flavula, Dichanthelium commutatum, Dioscorea villosa, Fragaria virginiana, Galium aparine, Geranium maculatum, Houstonia caerulea, Hypoxis hirsuta, Lobelia inflata, L. spicata, Lysimachia ciliata, Maianthemum racemosum, Osmorhiza longistylis, Parthenocissus quinquefolia, Podophyllum peltatum, Rubus phoenicolasius, Sanguinaria canadensis, Smilax rotundifolia, Solidago caesia, S. ulmifolia, Symphyotrichum patens, Taenidia integerrima, and Thalictrum thalictroides. 


\section{Open Fields and Fencerows}

Open fields and fencerows make up a large part of the valley lowlands. Some of this land was originally used in agriculture, but is no longer active except for infrequent haying. Other fields left completely fallow soon reach later stages of succession.

Woody species that usually become well established in these areas include Elaeagnus umbellata, Juniperus virginiana, Morus alba, M. rubra, and Rhamnus lanceolata. Grass species regularly dominate the herbaceous vegetation in fields and commonly include Andropogon virginicus, Dactylis glomerata, Poa species, Setaria pumila, and Tridens flavus. Frequent occurrences of Asteraceae species are also typical. Common plants are Achillea millefolium, Conyza canadensis, Hieracium caespitosum, Rudbeckia hirta, Solidago juncea, Symphyotrichum pilosum, and Vernonia noveboracensis. Other standard species include Apocynum cannabinum, Asparagus officinalis, Buglossoides arvensis, Coronilla varia, Daucus carota, Echium vulgare, Galium mollugo, Papaver dubium, Ranunculus bulbosus, Rubus pensilvanicus, Trifolium pratense, and T. repens.

\section{Disturbed Sites}

Many of the collections made in disturbed areas were collected along roadsides, but other sites in this category are cultivated lawns, recently disturbed lots, and land with frequent agricultural use. These areas are characterized by human-related activities or development, and share many species common to fields and fencerows.

Familiar woody plants of disturbed sites are Ailanthus altissima, Ligustrum sinense, and Rhus glabra, but most of the vegetation is comprised of herbaceous plants. Early in the season, several species of Brassicaceae dominate roadsides, waste places and 
agricultural fields. Some of these plants are Barbarea vulgaris, Capsella bursa-pastoris, Lepidium campestre, and Microthlaspi perfoliatum. The families of Asteraceae, Fabaceae and Lamiaceae contribute the most to disturbed areas. Common species in Fabaceae are Lathyrus latifolius, Lespedeza cuneata, Melilotus officinalis, Trifolium arvense, and T. campestre. In Lamiaceae are Glechoma hederacea, Lamium amplexicaule, L. purpureum, Nepeta cataria, and Prunella vulgaris. Prevalent species of Asteraceae are Ambrosia artemisiifolia, Carduus acanthoides, Centaurea biebersteinii, Chondrilla juncea, Cichorium intybus, Cirsium vulgare, Erigeron philadelphicus, Eupatorium altissimum, Lactuca serriola, Leucanthemum vulgare, Taraxacum officinale, Tragopogon dubius, Tussilago farfara, and Verbesina alternifolia.

Other herbaceous species of disturbed sites include Allium vineale, Anagallis arvensis, Asclepias syriaca, Chamaesyce nutans, Conium maculatum, Digitaria ischaemum, Duchesnea indica, Euphorbia corollata, Geranium molle, Hypericum perforatum, Lonicera japonica, Malva neglecta, Oenothera parviflora, Oxalis stricta, Penstemon canescens, Persicaria longiseta, Physalis longifolia, Phytolacca americana, Plantago lanceolata, P. rugelii, Rosa carolina, R. multiflora, Rumex obtusifolius, Saponaria officinalis, Silene latifolia, Solanum carolinense, Sorghum halepense, Stellaria media, Toxicodendron radicans, Verbascum thapsus, and Veronica persica.

\section{Aquatic and Bog Sites}

Aquatic species occur in ponds and rivers throughout the county. A few sites are described as bogs, including Mud Hole Bog in the northern Massanutten Mountains of the Fort Valley area. Plants of wet or boggy sites include many species of sedges and rushes such as Carex intumescens, Eleocharis obtusa, Juncus effusus, and Scirpus 
pendulus. Other plants of wet or aquatic areas include Alisma subcordatum, Caltha palustris, Equisetum arvense, Glyceria striata, Justicia americana, Lemna minor, Nasturtium officinale, Typha latifolia, and Veratrum virginicum.

\section{Floodplain and Riparian Zones}

The primary floodplain of Shenandoah County lies along the North Fork of the Shenandoah River. These low, flat areas are subject to occasional flooding and are typically composed of alluvial deposits. Floodplains may be open or wooded, but often contain a riparian zone if open. Woody vegetation commonly includes Acer negundo, Chionanthus virginicus, Platanus occidentalis, Salix nigra, Staphylea trifolia, Tilia americana, and Ulmus rubra. Typical herbaceous species are Chelone gabra, Cicuta maculata, Commelina communis, Conoclinium coelestinum, Cryptotaenia canadensis, Impatiens capensis, Mertensia virginica, Viola cucullata, V. sororia, and V. striata.

\section{Shale Barrens}

Shale barrens form a unique habitat in the region and occur largely in the Ridge and Valley Province. Most barrens are derived from the Devonian age Brallier formation. However, in Shenandoah County which includes parts of the Massanutten Mountains, some shale barrens are derived from the Martinsburg formation of the Upper Ordovician age. The barrens are characterized by outcrops of shale fragments on southward facing slopes (Platt 1951).

Vegetation is usually sparse, but this habitat supports several species. Common plants identified in this survey are Antennaria virginica, Arabis laevigata, Calystegia spithamaea, Cheilanthes lanosa, Cunila origanoides, Draba ramosissima, Eriogonum 
allenii, Opuntia humifusa, Packera antennariifolia, Paronychia montana, Phlox

subulata, Selaginella rupestris, and Viola pedata.

\section{Summary}

In 2011, 600 collections were made from March 21 - October 27, and in 2012, 50 collections were made from March 22 - May 30. A total of 650 new field collections were made during the study period (Fig. 8). Six specimens inadvertently collected outside the county boundary were removed from the survey. Overall, the 644 collections of Shenandoah County represent 518 species in 340 genera and 105 families. The herbaria search resulted in the inclusion of 1,492 specimens. These specimens represent 727 species in 394 genera and 120 families. An additional 404 species were not substantiated by the present survey but are recognized by the Digital Atlas of the Virginia Flora (2012) as present in the county. The combined results of the field collections, herbarium specimens, and the Digital Atlas make up a total of 1,299 species in 563 genera and 143 families. 


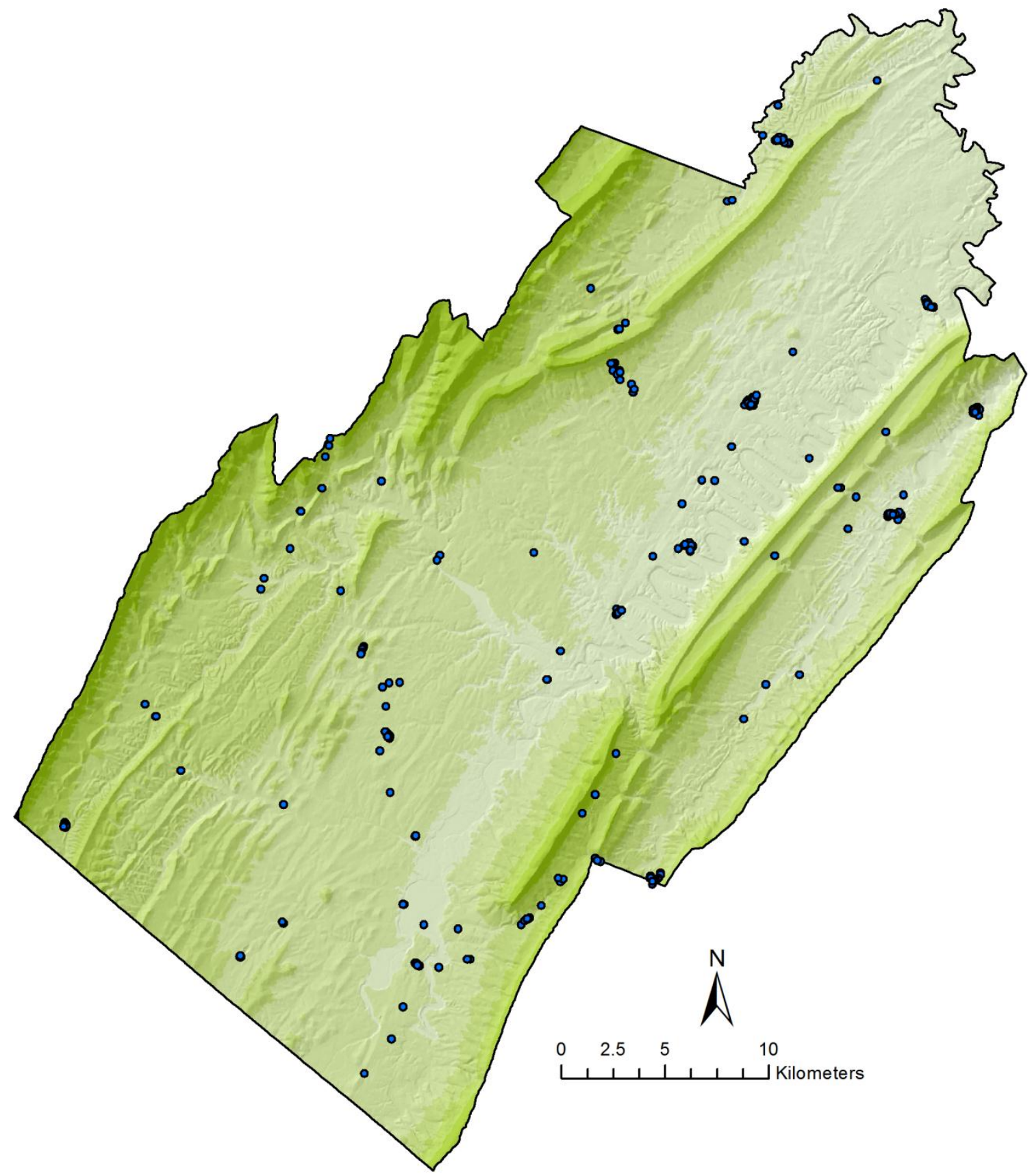

Figure 8. Collection sites and topography (USGS 2009) of Shenandoah County, Virginia. A total of 650 collections were made during the study period. 
A summary of Shenandoah County families is reported in Table 2. Of 1,299

species, 57 are ferns and fern allies, 8 are gymnosperms, 322 are monocots and 912 are

dicots. The most common family in the county is Asteraceae with 164 associated species.

Other large families, including number of species, are Poaceae (121), Cyperaceae (92),

Fabaceae (61), Rosaceae (56), Lamiaceae (42), Brassicaceae (37), Liliaceae (33),

Ranunculaceae (33), Scrophulariaceae (33), and Apiaceae (26). Between new collections

and herbarium specimens, 53 species are new records for the county, including 2 new

records for the state. Of all 1,299 species, 1,014 are native to Virginia, 268 are

introduced, and 14 are unspecified by the Digital Atlas.

Table 1. Taxonomic summary of plant families, including resources from new field collections, preexisting herbarium specimens, and Digital Atlas of the Virginia Flora records. Families are grouped by (a) ferns and fern allies, gymnosperms and monocots, and (b) dicots.

\begin{tabular}{|c|c|c|c|c|c|c|c|c|c|}
\hline a) & Family & & \multirow[t]{2}{*}{ Family } & \multicolumn{2}{|c|}{$G^{e^{r^{e^{2}}}}$ Se } & \multirow[t]{2}{*}{ Family } & \multicolumn{2}{|c|}{$G^{e^{n}}$ se } \\
\hline \multicolumn{8}{|c|}{ Ferns andFern Allies } & & \\
\hline & Aspleniaceae & 1 & 6 & Isoetaceae & 1 & 3 & Pteridaceae & 3 & 5 \\
\hline & Azollaceae & 1 & 1 & Lycopodiaceae & 6 & 8 & Salviniaceae & 1 & 1 \\
\hline & Dennsta edtiaceae & 2 & 2 & Ophioglossaceae & 2 & 7 & Selaginellaceae & 1 & 2 \\
\hline & Dryopteridaceae & 7 & 11 & Osmundaceae & 1 & 3 & Thelypteridaceae & 2 & 3 \\
\hline & Equisetaceae & 1 & 3 & Polypodiaceae & 2 & 2 & & & \\
\hline \multicolumn{10}{|c|}{ Gymnosperms } \\
\hline & Cupressaceae & 1 & 2 & & & & & & \\
\hline & Pinaceae & 2 & 6 & & & & & & \\
\hline \multicolumn{10}{|c|}{ Monocots } \\
\hline & Acoraceae & 1 & 1 & Hydrocharitaceae & 1 & 2 & Poaceae & 51 & 121 \\
\hline & Agavaceae & 1 & 1 & Iridaceae & 3 & 4 & Pontederiaceae & 1 & 1 \\
\hline & Alismataceae & 2 & 2 & Juncaceae & 2 & 16 & Potamogetonaceae & 2 & 6 \\
\hline & Araceae & 3 & 4 & Lemnaceae & 2 & 4 & Smilacaceae & 1 & 4 \\
\hline & Commelinaceae & 2 & 3 & Liliaceae & 19 & 33 & Sparganiaceae & 1 & 1 \\
\hline & Cyperaceae & 10 & 92 & Najadaceae & 1 & 1 & Typhaceae & 1 & 1 \\
\hline & Dioscoreaceae & 1 & 2 & Orchidaceae & 13 & 22 & Zannichelliaceae & 1 & 1 \\
\hline
\end{tabular}




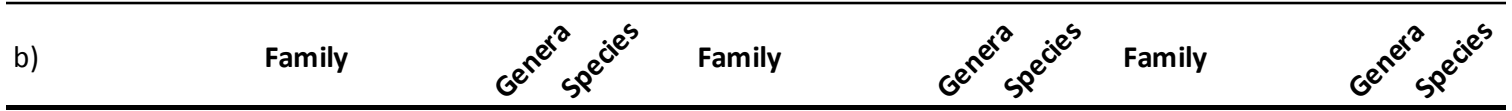

Dicots

\begin{tabular}{|c|c|c|c|c|c|c|c|c|}
\hline Acanthaceae & 2 & 5 & Droseraceae & 1 & 1 & Passifloraceae & 1 & 1 \\
\hline Aceraceae & 1 & 8 & Ebenaceae & 1 & 1 & Paulowniaceae & 1 & 1 \\
\hline Adoxaceae & 2 & 6 & Elaeagnaceae & 1 & 1 & Penthoraceae & 1 & 1 \\
\hline Amaranthaceae & 1 & 4 & Ericaceae & 10 & 16 & Phrymaceae & 1 & 1 \\
\hline Anacardiaceae & 2 & 6 & Euphorbiaceae & 5 & 13 & Phytolaccaceae & 1 & 1 \\
\hline Annonaceae & 1 & 1 & Fabaceae & 27 & 61 & Plantaginaceae & 1 & 5 \\
\hline Apiaceae & 18 & 26 & Fagaceae & 3 & 18 & Platanaceae & 1 & 1 \\
\hline Apocynaceae & 2 & 3 & Fumariaceae & 2 & 5 & Polemoniaceae & 1 & 5 \\
\hline Aquifoliaceae & 1 & 3 & Gentianaceae & 3 & 5 & Polygalaceae & 1 & 4 \\
\hline Araliaceae & 1 & 4 & Geraniaceae & 2 & 6 & Polygonaceae & 5 & 18 \\
\hline Aristolochiaceae & 2 & 2 & Grossulariaceae & 1 & 2 & Portulacaceae & 2 & 2 \\
\hline Asclepiadaceae & 3 & 13 & Haloragaceae & 1 & 1 & Primulaceae & 5 & 9 \\
\hline Asteraceae & 63 & 164 & Hamamelidaceae & 1 & 1 & Pyrolaceae & 2 & 4 \\
\hline Balsaminaceae & 1 & 2 & Hippocastanaceae & 1 & 1 & Ranunculaceae & 12 & 33 \\
\hline Berberidaceae & 4 & 4 & Hydrangeaceae & 2 & 2 & Rhamnaceae & 2 & 2 \\
\hline Betulaceae & 5 & 7 & Hydrophyllaceae & 2 & 2 & Rosaceae & 17 & 56 \\
\hline Bignoniaceae & 2 & 3 & Juglandaceae & 2 & 7 & Rubiaceae & 6 & 19 \\
\hline Boraginaceae & 8 & 10 & Lamiaceae & 22 & 42 & Rutaceae & 2 & 2 \\
\hline Brassicaceae & 22 & 37 & Lauraceae & 2 & 2 & Salicaceae & 2 & 13 \\
\hline Cactaceae & 1 & 1 & Lentibulariaceae & 1 & 1 & Santalaceae & 1 & 1 \\
\hline Callitrichaceae & 1 & 1 & Linaceae & 1 & 4 & Saururaceae & 1 & 1 \\
\hline Campanulaceae & 4 & 9 & Lythraceae & 1 & 1 & Saxifragaceae & 4 & 5 \\
\hline Cannabaceae & 1 & 2 & Magnoliaceae & 2 & 3 & Scrophulariaceae & 15 & 33 \\
\hline Caprifoliaceae & 3 & 10 & Malvaceae & 4 & 7 & Simaroubaceae & 1 & 1 \\
\hline Caryophyllaceae & 11 & 23 & Menispermaceae & 1 & 1 & Solanaceae & 4 & 9 \\
\hline Celastraceae & 3 & 5 & Molluginaceae & 1 & 1 & Staphyleaceae & 1 & 1 \\
\hline Chenopodiaceae & 1 & 4 & Moraceae & 3 & 4 & Tiliaceae & 1 & 1 \\
\hline Cistaceae & 2 & 3 & Myricaceae & 1 & 1 & Ulmaceae & 2 & 5 \\
\hline Clusiaceae & 1 & 7 & Nelumbonaceae & 1 & 1 & Urticaceae & 5 & 6 \\
\hline Convolvulaceae & 3 & 7 & Nymphaeaceae & 2 & 2 & Valerianaceae & 1 & 1 \\
\hline Cornaceae & 1 & 6 & Nyssaceae & 1 & 1 & Verbenaceae & 2 & 4 \\
\hline Crassulaceae & 2 & 5 & Oleaceae & 4 & 7 & Violaceae & 1 & 17 \\
\hline Cucurbitaceae & 1 & 1 & Onagraceae & 5 & 14 & Viscaceae & 1 & 1 \\
\hline Cuscutaceae & 1 & 3 & Orobanchaceae & 3 & 3 & Vitaceae & 2 & 4 \\
\hline Diervillaceae & 1 & 1 & Oxalidaceae & 1 & 6 & & & \\
\hline Dipsacaceae & 1 & 1 & Papaveraceae & 3 & 3 & & & \\
\hline
\end{tabular}


Although only naturalized species were targeted for collection, some new records for the county may have originated in cultivation. Three plants were collected from a churchyard at the same location: Aesculus glabra, Leavenworthia uniflora, and Sedum sarmentosum. On the property was A. glabra, a new record for the state as well as the county. This large tree was probably planted, but several saplings were established in the adjacent field including one large enough to bear flowers. Even more atypical to the region is $L$. uniflora, collected from the edge of the church parking lot. The nearest states where this plant is known are Ohio, Kentucky and Tennessee (USDA, Natural Resources Conservation Service 2012). S. sarmentosum was also collected near the parking lot but was observed on rock outcrops in the surrounding area as well. Other new records that are commonly cultivated include Forsythia suspensa, Euonymus alatus, Lunaria annua and Hibiscus syriacus. However, it is difficult to determine the history of the collected specimens.

All records are listed in the species catalog (Appendix A). The catalog is sorted alphabetically by family, genus, and species name within four higher taxonomic groups: ferns and fern allies, gymnosperms, monocots, and dicots. Each species, along with its common name when available, is followed by a list of collection records. Descriptions for new field collections include the date and location followed by the collector's name and collection number. Descriptions from pre-existing herbarium specimens may include all or parts of this information, depending on the completeness of the original record, and are followed by the herbarium code (JMUH, BDWR, HAVI, or LFCC). Non-native species are marked with an asterisk (*) after the scientific name. Species that are new additions to the county record are tagged with a plus (+) before the species name. Species 
documented by the Digital Atlas of the Virginia Flora but not identified by the present survey are preceded by a diamond $(\diamond)$ in the catalog. These species are not followed by a list of supporting specimens.

As time and environmental factors change, species distribution changes as well. The floristic survey of Shenandoah County, Virginia has been an update to the knowledge of the county flora. The results add 53 new county records to Shenandoah, including 2 new state records to Virginia. A total of 1,299 species are now recognized for the county, representing 563 genera and 143 families. The voucher specimens produced by new field collections are housed in the James Madison University Herbarium. 
Appendix A: Species Catalog. The catalog includes references to new field collections, pre-existing herbarium specimens, and Digital Atlas of the Virginia Flora records. Descriptions from new field collections are followed by the collector(s) name and collection number. Descriptions from herbarium specimens are followed by the herbarium code (JMUH, LFCC, BDWR, or HAVI). Species known from the Digital Atlas of the Virginia Flora but not substantiated by new collections or herbarium specimens are preceded by a diamond $(\diamond)$. Non-native species are represented by an asterisk $(*)$ following the species name. New records for Shenandoah County are preceded by a plus (+).

\section{Ferns \& Fern Allies}

\section{Aspleniaceae}

Asplenium montanum Willd. (mountain spleenwort)

- $\quad$ Wild Cat Hollow; Sandstone Cliffs; Altitude 1775.; 3/1/1950; L. Artz (JMUH)

Asplenium platyneuron (L.) B.S.P. (ebony spleenwort)

- $\quad$ Growing in forest on mountain slope among leaf litter.; 5/11/2011; 38.95333,-78.52975;

Domangue, C.K. McMullen 200

- $\quad$ Rt 675 3.7mi E of Rt 11 jct, E of Edinburg; Devonian shale barren, outcropping, SE facing slope.; 4/12/1982; R. Atkinson 7 (JMUH)

- $\quad$ Elizabeth Furnace Picnic Area George Washington National Forest; Moist, rocky banks-abundant in limestone areas.; 8/21/1978; S.M. Collins (LFCC)

- $\quad$ Liberty Furnace, Shale Barrens \#717/690.; 9/7/1975; R. \& H. Simpson (LFCC)

Asplenium resiliens Kunze (blackstem spleenwort)

- $\quad$ Pugh's Run; Limestone Sink hole; Altitude 900.; 8/16/1959; L. Artz (JMUH)

Asplenium rhizophyllum L. (walking fern)

- $\quad 1.2$ miles south of Orkney Springs at Seven Springs.; 9/9/1979; R.C. Simpson (LFCC)

- $\quad$ At Fishers Hill on Rt \#757/601 on Tumbling Run Creek; on limestone (N-facing) outcrop on Tumbling Run (across from Ritenours Grocery Store) limeston covered with moss. A platyneuron and Pellaea atropurpurea were also present. Another colony of C. rhizophyllum is found ca. 1/2 mi downstream.; 9/6/1975; K.D. Ramsey (LFCC)

Asplenium ruta-muraria L. (wallrue)

- $\quad$ Pugs Run; Limestone Cliffs; Altitude 800.; 9/29/1949; L. Artz (JMUH)

- $\quad$ Rte. 663, N. of Woodstock .2 mi. from Rte. 11; Limestone cliffs, southwest exposure. Not abundant, but frequent in local area.; 11/16/1979; N. Bodkin, M. Kovalchick (JMUH)

- $\quad$ Fishers Hill on Rt \#601 on W facing limestone outcrop along Tumbling Run; limestone outcrop along mt. stream; At least 45 clumps were counted in a 1/2 mi. area.; 9/3/1975; K.D. Ramsey (LFCC)

Asplenium trichomanes L. (maidenhair spleenwort)

- $\quad$ Camp Caroline Furnace, Fort Valley (7 miles S.E. of Edinburg on Route 675); Cliffs above west bank of Passage Creek, 100 yards north of Cabin Bridge; Large, shady rock on shale cliff.; 4/10/1979; B.E. Brandt 9 (JMUH)

- $\quad$ Rt. 628 at Cedar of 780 ft.; rock on creek bank.; 11/2/1975; E. Fowlkes, (LFCC) 


\section{Azollaceae}

$\diamond$ Azolla caroliniana Willd. (Carolina mosquitofern)

Dennstaedtiaceae

Dennstaedtia punctilobula (Michx.) T. Moore (eastern hayscented fern)

- $\quad$ Tomahawk Pond Campground, beside dirt trail in wooded area.; 8/8/2011; 38.75927,-78.84100; Domangue 531

- $\quad$ FR 274 East side.; 7/22/1981; D.E. Miller 8 (JMUH)

- $\quad$ Roadside; 1 mi. South of Kings Crossing on Rt. 678.; 10/3/1975; R. Brown (LFCC)

- $\quad 3$ miles East of Strasburg; 100 yds. South of Rt. 55 towards Signal Knob; Growing along old roadside.; 10/28/1978; M.J. Hughes, B. Legge (LFCC)

Pteridium aquilinum (L.) Kuhn (western brackenfern)

- $\quad$ Growing on the edge of dirt road in forest.; 5/11/2011; 38.94842,-78.52805; Domangue, C.K. McMullen 204

- $\quad$ Rt. 608 at Columbia Furnace, along roadside which leads to Wolf Gap; 10/19/1978; M.L. Snarr (LFCC)

- $\quad$ George Washington National Forest Rt. 6781.7 miles north of the Day Use Area at Elizabeth Furanace; Broadleaf, deciduous, forest, oak-hickory, roadside; Elev. 875 ft.; 5/16/1978; L. Frey, J. Brumback, R. Whiting, K. Halstead (LFCC)

Dryopteridaceae

Athyrium filix-femina (L.) Roth ex Mert. (common ladyfern)

- $\quad$ 1/2 mile East of Intersection mine Road and Powell Fort Road George Washington National Forest; Cove.; 5/5/1977; L. Valines (LFCC)

- $\quad$ Massanutten Mt., Little Fort Recreation Area, along Powell's Mt. Trail (to Edinburg Gap) about 1 mi. south of the Little Fort Camping Area; Elev. 1390 ft.; 6/26/1977; R.C. Simpson, G. Evans, D. Steed (LFCC)

Cyrtomium falcatum (L.f.) K. Presl (Japanese netvein hollyfern) *

- 1 mile North of Woodstock, east on \#663 to the second bridge, North approx. 75 yds. on Pugh's Run; shaded limestone bank on Pugh's Run; Associates: Asplenium chizophyllus, Aseyptoles; Elev. 800'; About 25 plants scattered along the stream bank.; 5/20/1974; R.C. Simpson (LFCC)

- 1 mile north of Woodstock, east on \#663 to the second bridge, north approx 75 yds on Pugh's Run; shaded limestone bank on Pugh's Run; Associates: Walking Fern Asplenium rhizophyllus, Wall Rue Asplenium cryptolepis; Elev. 800'.; 9/20/1976; R.C. Simpson, M. Kelly (LFCC)

Deparia acrostichoides (Sw.) M. Kato (silver false spleenwort)

- $\quad$ Poter Mill Bog; Bog.; 6/16/1978; R.C. Simpson (LFCC)

Dryopteris carthusiana (Vill.) H.P. Fuchs (spinulose woodfern)

- $\quad$ Fort Valley Peter's Mill Bog.; 6/24/1980; R.C. Simpson (LFCC)

+ Dryopteris cristata (L.) Gray (crested woodfern)

- $\quad$ Mud hole Gap, Fort Valley; Elev. 1500 ft.; 9/14/1979; R.C. Simpson (LFCC) 
Dryopteris intermedia (Muhl. ex Willd.) Gray (intermediate woodfern)

- $\quad 3$ miles east of Strasburg 100 yds. South of Rt 55; Rich Oak-Hickory woods.; 10/29/1978; M.J. Hughes, B. Legge (LFCC)

- Fort Valley at Elizabeth Furnace, Signal Knob Trail; Rocky Hillside.; 10/15/1975; J.Fitzgerald (LFCC)

Dryopteris marginalis (L.) Gray (marginal woodfern)

- $\quad$ Rt. 730 roadside on steep bank at forest border.; 8/23/2011; 38.73852,-78.54751; Domangue 575

- Camp Roosevelt, along horse trail in wooded area.; 4/26/2011; 38.72922,-78.51283; Domangue, M. Lantz 114

- $\quad 1.2$ miles east from the North fork of the Shenandoah River, rte. 758; Habitat - woods.; 4/12/1982; C. Yost 21 (JMUH)

- Fort Valley, On shale barrens by Forestry Departments Cabin. 5 miles south on Route 678; Elev. 1000 feet.; 4/25/1980; L. Stewart (LFCC)

- $\quad$ George Washington National Forest, Elizabeth Furnace Camp Ground; Oak Hickory.; 10/11/1975; W. Bridwell (LFCC)

Onoclea sensibilis L. (sensitive fern)

- $\quad 2.7$ mi. from Jct. 678 Rt. 771, Forest Rd. 273 Mudhole Bog; moist, swamp like area.; 5/18/1983; M.L. Eger, S. Shull 17 (JMUH)

- $\quad$ Fort Valley Powell's Fort Camp; Low wet area beside Little Stony Creek.; 9/29/1975; J.Fitzgerald (LFCC)

- $\quad$ Elizabeth Furnace picnic ground; George Washington National Forest; along stream bank.; 8/21/1978; S.M. Collins (LFCC)

Polystichum acrostichoides (Michx.) Schott (Christmas fern)

- Devil's Backbone State Forest, on the bank of shaded stream.; 7/20/2011; 39.04845,-78.43222; Domangue, C.K. McMullen 469

- Conicville, in woods among leaf litter.; 5/20/2011; 38.83309,-78.67276; Domangue 239

- $\quad$ FR 274 West side.; 7/8/1981; D.E. Miller 46 (JMUH)

- Fort Valley Powell's Fort Camp; Roadside ditch; very abundant.; 9/29/1975; J.Fitzgerald (LFCC)

- By parking lot area - Signal Knob parking lot area; deciduous woods; Elev. 600 ft.; 5/17/1978; H.E. Reed (LFCC)

Woodsia ilvensis (L.) R. Br. (rusty woodsia)

- Cedar Cr.; Shale cliffs; Altitude 1000 ft.; 9/19/1955; L. Artz (JMUH)

- 1 mile of liberty furnace junction of 717 and 690; Shale Barrens; Elev. 1500 ft.; 9/7/1975; R.C. Simpson (LFCC)

Woodsia obtusa (Spreng.) Torr. (bluntlobe cliff fern)

- $\quad$ Approx 5 mi E. of Edinburg then S of \#678 on a shale barren South of road; Elev. $2300 \mathrm{ft}$; 7/7/1978; R.C. Simpson (LFCC)

- Powells Camp Road; Bog.; 6/27/1975; R.C. Simpson (LFCC) 
Equisetaceae

Equisetum arvense L. (field horsetail)

- Tomahawk Pond Campground, growing in pond.; 10/27/2011; 38.75840,-78.84048; Domangue, 656

- $\quad$ Fort Valley area, where Rt. 775 turns off 678, beside Narrow Passage Creek.; 4/24/1978; C. Hisey (JMUH)

- $\quad$ Fort Valley; 4/9/1977; R.C. Simpson (LFCC)

$\diamond$ Equisetum hyemale L. ssp. affine (Engelm.) Calder \& R.L. Taylor (scouringrush horsetail)

Equisetum sylvaticum L. (woodland horsetail)

- $\quad$ Mudhole Bog; Altitude 1200 ft.; 7/3/1948; L. Artz (JMUH)

Isoetaceae

$\diamond$ Isoetes engelmannii A. Braun (Appalachian quillwort)

$\diamond$ Isoetes lacustris L. (lake quillwort)

$\diamond$ Isoetes valida (Engelm.) Clute (strong quillwort)

\section{Lycopodiaceae}

Dendrolycopodium hickeyi (W.H. Wagner, Beitel \& Moran) A. Haines (rare clubmoss)

- Massanutten Mountain Little Fort recreation Area along Powell's mountain Trail (to Edinburg Gap) about 1 mi South of the little fort camping Area; Along Dry area of jeep trail.; 6/26/1977; G. Evans, D. Steed (LFCC)

Dendrolycopodium obscurum (L.) A. Haines (Pennsylvania clubmoss)

- Camp Roosevelt, near gravel lot in woods. Sandy soil.; 7/29/2011; 38.72784,-78.51546; Domangue 485

Diphasiastrum digitatum (Dill. ex A. Braun) Holub (fan clubmoss)

- Camp Roosevelt, near gravel lot in woods. Sandy soil.; 7/29/2011; 38.72784,-78.51546; Domangue 486

- $\quad$ Little Fort; Pine Woods; Altitude 1000 ft.; 9/13/1963; L. Artz (JMUH)

- Camp Caroline Furnace, Fort Valley, (7 miles S.E. of Edinburg on Route 675). 200 yards along Spring Trail; Rich, damp woods.; 4/10/1979; B.E. Brandt 10 (JMUH)

- Rt. 691; Bear Oak Prescribed Burn, 300 yds north on Laurel Run Trail off Rt. \#691; Elev. 3005 feet.; 5/16/1981; S. Collins (LFCC)

- $\quad$ Big Springs Fort Valley; sterile hillside.; 9/3/1975; R. \& H. Simpson (LFCC)

$\diamond$ Diphasiastrum tristachyum (Pursh) Holub (deeproot clubmoss)

Huperzia lucidula (Michx.) Trevis. (shining clubmoss)

- $\quad$ Mine Run; Moist Woods; Altitude 800 ft.; 5/20/1963; L. Artz (JMUH)

- Ft. Valley 2 mi E of Detrick on Rt \#758. "Milford Gap"; beside and growing on rocks in a mountain stream through "Milford Gap".; 8/16/1975; K.D. Ramsey (LFCC) 
$\diamond$ Lycopodiella $\times$ gilmanii A. Haines

Lycopodium clavatum $\mathrm{L}$. (running clubmoss)

- $\quad$ Camp Roosevelt, near gravel lot in woods. Sandy soil.; 7/29/2011; 38.72784,-78.51546; Domangue 487

- Bear Oak Prescribed Burn, 300 yards north on Laurel Run Trail, off Rt \#691; along bank of a natural pond.; 5/22/1981; L. Dotson, J. Hepner, S. Collins (LFCC)

- Ft. Valley on Rt. \#772 to Powells Ft. Camp; deciduous/mixed woods on E. side of road.; 9/19/1975; K.D. Ramsey (LFCC)

$\diamond$ Spinulum annotinum (L.) A. Haines

Ophioglossaceae

Botrychium dissectum Spreng. (cutleaf grapefern)

- $\quad$ Mine Run; Dry Woods; Altitude 1000 ft.; 9/13/1963; L. Artz (JMUH)

Botrychium matricariifolium (Doll) A. Braun ex Koch (matricary grapefern)

- Approx 6 miles west of Columbia Furnace on Laurel Run at the intersection of Rt. 691 and Rt. 717.2 miles west on Rt. 691 (in seepage ponds); At base of Va. Pine in shale, very little competition on ground.; 6/5/1977; R.C. Simpson, K.D. Ramsey (LFCC)

Botrychium oneidense (Gilbert) House (bluntlobe grapefern)

- Aprox 6 miles West of Columbia Furnace At Laurel Run, .2 miles West on public access road off Rt. 691; Moist, Low Area, in Liriodendron - Ulmus Swano; Blue-green color.; 6/5/1977; R.C. Simpson, K.D. Ramsey (LFCC)

$\diamond$ Botrychium simplex E. Hitchc. (little grapefern)

Botrychium virginianum (L.) Sw. (rattlesnake fern)

- $\quad$ Tomahawk Pond Campground, beside dirt trail in woods.; 6/8/2011; 38.75943,-78.84088; Domangue 308

- Conicville, in woods among leaf litter.; 5/20/2011;38.83309,-78.67276; Domangue 238

- Mine Run; Dry Woods; Altitude $1000 \mathrm{ft}+$ or -.; 5/20/1963; L. Artz (JMUH)

- $\quad 1 / 2$ mile East of interstate Nine on Mountain Road and Powells Fort Road; Cove in George Washington National Forest.; 4/27/1977; L. Valines (LFCC)

- Beside parking lot at Signal Knob Trail; Deciduous Forest; Elev. 600.; 5/22/1978; H.E. Reed (LFCC)

$\diamond$ Ophioglossum engelmannii Prantl (limestone adderstongue)

Ophioglossum vulgatum $\mathrm{L}$. (southern adderstongue)

- $\quad$ Big Spring; Calcareous Woods; Altitude 1400 ft.; 8/22/1950; L. Artz (JMUH)

- Northern Massanutten Mountain Range, Powell's Fort Valley, Mudhole Bog. Growing in extremely wet conditions in an acidic sphagnum bog between two drainage areas. Elv. 1300'. Small population associated with a heavy ground cover typical of such northern -like habitats.; 7/15/1976; B.E. Diven, N.L. Bodkin 761a, 762a, 763, 765c, 766c (JMUH) 


\section{Osmundaceae}

Osmunda cinnamomea L. (cinnamon fern)

- $\quad$ Northern Massanutten Mountains; Mudhole Bog.; 7/5/1978; J. Huffman, B. Latham, D. Stoltzfus 17 (JMUH)

- $\quad$ Ft. Valley on Rt \#771; "cove forest" mountain stream with Tsuga canadansis; unfertile frond.; 9/21/1975; K.D. Ramsey (LFCC)

Osmunda claytoniana L. (interrupted fern)

- $\quad$ Rt 789 (Rue Road); Elev. 2000 ft.; 5/20/1980; S. Gwyn (LFCC)

Osmunda regalis L. (royal fern)

- $\quad$ Roadside Rt. 675 West of King's Crossing.; 5/17/1988; J. Seguin 17 (JMUH)

- $\quad$ Northern Massanutten Mountains; Mudhole Bog.; 7/5/1978; J. Huffman, B. Latham, D. Stoltzfus 16 (JMUH)

- $\quad$ Ft. Valley, Rt. 675; Swampy and wet area.; 5/21/1986; B. Kerr, B. Rosson 18 (JMUH)

- $\quad$ 1/2 Mile up on Veach Gap trail (Fort Valley Side) on left side of trail in seepage Area; 1000 Elev. Deciduous Forest(mostly oaks) Seepage area; Elev. 1000.; 5/21/1978; H.E. Reed (LFCC)

\section{Polypodiaceae}

$\diamond$ Pleopeltis polypodioides (L.) Andrews \& Windham ssp. michauxiana (Weath.) And. (resurrection fern)

Polypodium virginianum L. (rock polypody)

- $\quad 3.6$ miles east of Edinburg on Route 675 at the foot of a Devonian Shale barren - at Bowman's Crossing - Rocky bank (Originally was green, but darkened while being pressed).; 6/22/1977; K. Short (JMUH)

- $\quad 3.5$ miles east from Edinburg along Rt. 675.; 6/27/1978; J. Huffman, B. Latham, D. Stoltzfus 8 (JMUH)

- $\quad$ Camp Caroline Furnace, Fort Valley (7 miles S.E. of Edinburg on Route 675); cliffs above west bank of Passage Creek, 100 yards north of Cabin Bridge; Large, shady rock on shale cliff.; 4/3/1979; B.E. Brandt 7 (JMUH)

- $\quad$ Fort Valley.; 3/21/1975; R.C. Simpson (LFCC)

- $\quad$ Elizabeth Furnace Picnic area; Mill Race hall common on Rock.; 4/8/1977; L. Valines (LFCC)

Pteridaceae

Adiantum pedatum L. (northern maidenhair)

- $\quad$ Rt. 789 roadside, on bank at forest border.; 8/8/2011; 38.90214,-78.69392; Domangue 536

$\diamond$ Cheilanthes eatonii Baker (Eaton's lipfern) 
Cheilanthes lanosa (Michx.) D.C. Eat. (hairy lipfern)

- Woodstock, Riverview Park, on rocky ledge off Effinger Trail.; 9/29/2011; 38.87134,-78.49026; Domangue 651

- Woodstock, Riverview Park. On edge of Effinger Trail, rocky soil.; 3/29/2011; 38.87163, -78.49013; Domangue 70

- Fort Valley, Rt. 675; Rocky cliffs and shale barrens (Devonian shale).; 5/21/1986; B. Rosson, B. Kerr 16 (JMUH)

- $\quad$ Fort Valley area.; 5/18/1984; BIO 501133 (JMUH)

- $\quad$ Fort Valley shale barrens; Rt 675 northwest of Edinburg.; 5/17/1988; J. Seguin 18 (JMUH)

- $\quad 39.3$ mi. NE of J.M.U. Exit 71 off I-81, right on 675 E to Edinburg. Left on Main St., right on 675 E to Ft. Valley. Grows on shale outcrops with almost non-existent ground cover. Endemic plant.; 6/22/1977; K. Kingsley 5 (JMUH)

- $\quad$ Edinburg Gap; Shale Cliff; Altitude 2000 ft.; 8/8/1964; L. Artz (JMUH)

- $\quad 2$ 1/2 miles south of Detrick on route 678. Fort Valley; Shale bank.; 4/25/1983; T. Rexroad 1216 (JMUH)

- $\quad 3.0$ miles north of Kings Crossing on Rt. 678 on shale bank along roadside; Shale stone.; 4/25/1983; B. Kelley 9 (JMUH)

- $\quad$ Rte. 675 Bowmans Crossing, 3.5 miles from Edinburg. Sunny shale barren above roadside.; 7/2/1979; VA Flora Class 16 (JMUH)

- $\quad$ Rt. 678 at "Glass house."; 7/13/1975; R.C. Simpson (LFCC)

- $\quad$ Shale barrens at North end of Burnshire Bridge (Woodstock); Shale barren cliff.; 3/1/1998; J. Malcom (LFCC)

Pellaea atropurpurea (L.) Link (purple cliffbrake)

- Elizabeth Furnace Recreation Area, growing in rock crevice beside trail.; 8/23/2011; 38.92875, -78.32726; Domangue 581

- $\quad$ Rte. 663, N. of Woodstock .2 mi. from Rte. 11; Limestone cliffs, southwest exposure.; 11/16/1979; N. Bodkin, M. Kovalchick (JMUH)

- $\quad$ Fisher's Hill; Palmer's property.; 3/16/1975; R.C. Simpson (LFCC)

$\diamond$ Pellaea glabella Mett. ex Kuhn ssp. glabella (smooth cliffbrake)

Salviniaceae

$\diamond$ Salvinia molesta Mitchell (kariba-weed) *

$$
\text { Selaginellaceae }
$$

Selaginella apoda (L.) Spring (meadow spikemoss)

- $\quad$ Peter's Mill Run; Bog; Altitude 1600 ft.; 8/8/1964; L. Artz (JMUH)

- On Rt. \#678 approx 3 mi. N. of kings crossing; wet meadow along intermittent stream at base of shale barrens.; 5/3/1977; R.C. Simpson (LFCC)

- U.S. Rt. 111 mile of Edinburg Hockman Homestead $30 \mathrm{ft}$. west of dwelling front yard.; 9/30/1979; R.C. Simpson (LFCC) 
Selaginella rupestris (L.) Spring (northern selaginella)

- Rt 675 E of Rt 11 jct, E of Edinburg; Devonian shale barren, outcropping, SE facing slope.; 4/12/1982; R. Atkinson 7 (JMUH)

- $\quad$ Edinburg Gap; Shale Cliffs; Altitude 1500 ft.; 8/8/1964; L. Artz (JMUH)

- $\quad$ Fort Valley shale barrens; Rt 675 northwest of Edinburg.; 5/17/1988; J. Seguin 19 (JMUH)

- Shale barrens; 3.5 miles east from Edinburg along Rt. 675.; 6/27/1978; J. Huffman, B. Latham, D. Stoltzfus 6 (JMUH)

- $\quad$ Fort Valley, Rt. 675; Dry rocks and shale barrens.; 5/21/1986; B. Rosson, B. Kerr 14 (JMUH)

- $\quad$ Edinburg Gap shale barren, westside.; 7/12/1975; R.C. Simpson (LFCC)

\section{Thelypteridaceae}

Phegopteris hexagonoptera (Michx.) Fee (broad beechfern)

- $\quad$ Milford Gap, Fort Valley.; 9/20/1975; (LFCC)

- $\quad$ Milford Gap, Fort Valley; wet rocky wooded streamside.; 9/20/1975; R. \& H. Simpson (LFCC)

Thelypteris noveboracensis (L.) Nieuwl. (New York fern)

- Devil's Backbone State Forest. Growing beside forest road.; 6/8/2011; 39.04684,-78.42906; Domangue 313

- Northern Massanutten Mountains; Mudhole Bog.; 7/5/1978; J. Huffman, B. Latham, D. Stoltzfus 17 (JMUH)

- Elizabeth Furnace Picnic Area George Washington National Forest; moist, rich soil on stream bank.; 8/21/1978; S.M. Collins (LFCC)

Thelypteris palustris Schott (eastern marsh fern)

- $\quad$ Low, wet open woods; Fort Valley, Milford Gap.; 9/20/1975; R.C. Simpson (LFCC)

- $\quad$ Fort Valley, Milford Gap; low wet open woods.; 9/20/1975; R. \& H. Simpson (LFCC)

\section{Gymnosperms}

\section{Cupressaceae}

Juniperus virginiana $\mathrm{L}$. (eastern redcedar)

- Shenandoah Co. Park. Tree growing between lawn and forested area.; 5/18/2011; 38.93691, -78.45180; Domangue 233

- $\quad$ Route 764, past second house; Roadside.; 7/1/1987; Jennifer Estep (BDWR)

- George Washington National Forest, Elizabeth Furnace Picnic Area; field next to stream; Elev. 800 feet.; 9/20/1975; R.E. Keplinger (LFCC)

- $\quad$ Strasburg, Washington St at Lowwater bridge; edge of roadside woods.; 10/13/1979; R. Bowden (LFCC)

Thuja occidentalis L. (arborvitae)

- $\quad$ Aprox. 2 miles North of Woodstock off US RT, 11 at Pughs Run; on limestone Ledge above stream south facing slope.; 6/5/1977; R.C. Simpson, K.D. Ramsey (LFCC)

Pinaceae

Pinus echinata Mill. (shortleaf pine)

- $\quad$ Off U.S. Rt. 11 Woodstock, Va. Turn East on Mill Road for 1.6 miles, turn North on 758, 3.2 miles to the Woodstock tower area; mountain top; Elev. 1500 ft.; 9/15/1986; H. Fisher (LFCC) 
Pinus pungens Lamb. (Table Mountain pine)

- Camp Roosevelt, growing beside gravel lot. Large tree.; 6/24/2011; 38.72800,-78.51525;

Domangue, J. Courtwright 367

Pinus rigida Mill. (pitch pine)

- Go Rt 42-S for 5.7 miles until it intersects with Rt 675-W. Go west on 675 until you come to Wolf Gap recreation area. Specimens found along Big Blue hiking trail; Oak-hickory forest type. Dry moist conditions; Elev. 2800 ft.; 10/8/1978; L. Leta (LFCC)

- Property of James A. Hepner 300 yds north of the intersection of Rt \#604 and Rt \#623.; 10/5/1981; J.A. Hepner (LFCC)

Pinus strobus L. (eastern white pine)

- $\quad$ Elizabeth Furnace Recreation Area. Large tree growing in lawn.; 5/25/2011; 38.92837,-78.33027; Domangue 264

- $\quad$ Northern Massanutten Mountains; Mudhole Bog.; 6/27/1978; J. Huffman, B. Latham, D. Stoltzfus 35 (JMUH)

Pinus virginiana Mill. (Virginia pine)

- $\quad$ Tomahawk Pond Campground. Large tree at edge of parking lot.; 6/8/2011; 38.75854,-78.84187; Domangue 309

- 14 miles E. on Rt. 675. Shale Barren.; 5/18/1983; BIO 5014 (JMUH)

- Strasburg, Washington St at Lower water bridge then 25 yds east at roadside; roadside slightly woody, slightly shrubby.; 10/20/1979; R. Bowden (LFCC)

- Edinburg, Va .7 miles of 841 on 68. rt. Hand side of road; small wooded area.; 10/25/1976; J.S. McCarthy (LFCC)

Tsuga canadensis (L.) Carrière (eastern hemlock)

- $\quad$ Elizabeth Furnace Recreation Area. Large tree in lawn.; 6/24/2011; 38.92833,-78.33037; Domangue, J. Courtwright 378

- Fort Valley state road 774, 150 yards due East of Boldin residence; Elev. 1500 ft.; 11/5/1985; R. Graves (LFCC)

- Paddy's Run 4 mi W of Star Tannery off Rt 55; moist mountain stream area; Elev. 900 ft.; 10/9/1978; J. Macdonell (LFCC)

\section{Monocots}

Acoraceae

Acorus calamus L. (calamus) *

- 6 m. West of Columbia furnace on Laurel run at intersection of Rt 691 \& Route 717; In and at edge of slow flowing spring fed seepage stream.; 6/5/1977; R. Simpson, K. Ramsey (LFCC)

Agavaceae

Yucca filamentosa L. (Adam's needle)

- $\quad 1 / 4$ mi. W. of Rt. 628 at Cedar Cr; fields and roadsides; white ball like blooms; Elev. $900 \mathrm{ft}$.; 10/12/1975; E. Fowlkes (LFCC) 
Alismataceae

Alisma subcordatum Raf. (American water plantain)

- Tomahawk Pond Campground, on the edge of pond.; 8/8/2011; 38.75881,-78.84084; Domangue, 526

- $\quad$ Little Fort Valley; Bog; Altitude 1000 ft.; 9/13/1963; L. Artz (JMUH)

Sagittaria latifolia Willd. (broadleaf arrowhead)

- $\quad$ Passage Cr.; Water; Altitude 800.; 7/21/1965; L. Artz (JMUH)

Araceae

$\diamond$ Arisaema dracontium (L.) Schott (green dragon)

Arisaema triphyllum (L.) Schott (Jack in the pulpit)

- $\quad$ Fort Valley, beside Mine Mountain Rd. in moist, wooded environment.; 4/26/2011; 38.89643, -78.40716; Domangue, M. Lantz 125

- $\quad$ Mine Run; Moist Woods; Altitude 1100 ft.; 5/20/1963; L. Artz (JMUH)

- $\quad$ Northern Massanutten Mountains; Mudhole Bog.; 6/27/1978; J. Huffman, B. Latham, D. Stoltzfus 29 (JMUH)

- Camp Caroline Furnace, Fort Valley (7 miles SE of Edinburg on Route 675). Surrounding Old Mill ruins; Dry, rich woods.; 4/23/1979; B.E. Brandt 31 (JMUH)

- Powell's Fort seepage area, Mudhole Gap.; 6/24/1978; R.C. Simpson (LFCC)

- Fort Valley, Powell's Fort Camp bog where electric line cuts across wetlands; Open wet, Carex meadow dleared from deforestation along the power line.; 5/31/1977; R.C. Simpson (LFCC)

$\diamond$ Orontium aquaticum L. (goldenclub)

$\diamond$ Symplocarpus foetidus (L.) Salisb. ex W.P.C. Barton (skunk cabbage)

Commelinaceae

Commelina communis L. (Asiatic dayflower) *

- $\quad$ Rt. 720 roadside near Meems Bottom Covered Bridge.; 9/15/2011; 38.72065,-78.65437;

Domangue 625

- Public boat landing at N. Fork Shenandoah River. Growing beside gravel lot. Silty soil.; 7/1/2011; 38.84578,-78.52982; Domangue 409

- $\quad$ Near Fort Valley on Rt. 675, 38.5 mi. from Madison U.; Found on shale barren, roadside.; 6/22/1977; S.E. Grimes 83 (JMUH)

- $\quad$ Fort Valley junction Rt. \#678 and Rt. \#771; shale barrens.; 8/9/1975; R.C. Simpson (LFCC)

- Approx. 5 mi. E. of Edinburg then S. of Rt. \#678 natural shale barrier on the mountain S. of road; Elev. 2300 ft.; 7/7/1978; R.C. Simpson (LFCC)

$\diamond$ Commelina erecta $\mathrm{L}$. (whitemouth dayflower)

Tradescantia virginiana $\mathrm{L}$. (Virginia spiderwort)

- $\quad$ Blue Hole trail, Elizabeth furnace; Woods.; 5/16/1982; L. Michael Hill 4 (BDWR)

- $\quad$ Approx 5 mi East of Edinburg then S. of \#678 on a shale barren South of road; Elev. 2300.; 7/7/1978; R.C. Simpson (LFCC) 


\section{Cyperaceae}

$\diamond$ Bulbostylis capillaris (L.) Clarke in Hook. (densetuft hairsedge)

$\diamond$ Carex aestivalis M.A. Curtis ex Gray (summer sedge)

$\diamond$ Carex albicans Willd. ex Spreng. (whitetinge sedge)

$\diamond$ Carex albursina Sheldon (white bear sedge)

Carex amphibola Steud. (eastern narrowleaf sedge)

- Approx. 6 miles West of Columbia Furnace on Laurel Run at the intersection of Rt. 691 and Rt. 717.2 miles West on Rt. 691; Mesic Cove Forest.; 6/5/1977; R.C. Simpson, K.D. Ramsey (LFCC)

- $\quad$ Approx. 2 miles North of Woodstock on US Rt. 11 at Pugh's Run; over limestone at stream's edge.; 6/5/1977; R.C. Simpson, K.D. Ramsey (LFCC)

$\diamond$ Carex argyrantha Tuck. ex Dewey (hay sedge)

Carex atlantica Bailey (prickly bog sedge)

- Fort Valley Powell's fort Camp bog where the electric line cuts across the wet lands; Open wet meadow cleared from deforestation along the power line.; 5/31/1977; R.C. Simpson (LFCC)

- Approx. 6 miles West of Columbia Furnace at Laurel Run .2 miles West on public access road off Rt. 691; moist area along road.; 6/5/1977; R.C. Simpson, K.D. Ramsey (LFCC)

Carex baileyi Britton (Bailey's sedge)

- Fort Valley, 1/4 mile E. of Glass house.; 6/22/1980; R.C. Simpson (LFCC)

Carex blanda Dewey (eastern woodland sedge)

- Approximately 2 miles North of Woodstock off US Rt. 11 at Pugh's Run; over limestone at stream's edges.; 6/5/1977; R.C. Simpson, K.D. Ramsey (LFCC)

- On Rt. \#678 approx. 3 mi. N. of Kings Crossing; wet meadow along intermitent stream at base of shale barrens.; 5/3/1977; R.C. Simpson (LFCC)

Carex bromoides Schk. ex Willd. (brome-like sedge)

- Tomahawk Pond Campground, growing on the edge of the pond.; 5/10/2011; 38.75838,-78.84055; Domangue, B. Burkholder 164

- Peter Mill Bog; Bog.; 6/16/1978; R.C. Simpson (LFCC)

Carex caroliniana Schwein. (Carolina sedge)

- Approx. 2 mi. N.W. of the junction of \#675 and \#678 on \#675 on the Massanutten Mtn; Moist spring.; 5/4/1977; R.C. Simpson (LFCC)

- Massanutten Mountain, Little Fort Recreation Area, along Powell's mountain trail (to Edinburg Gap) about $1 \mathrm{mi}$. South of the Little Fort Camping Area; Along dry jeep trail.; 6/26/1977; R.C. Simpson, G. Evans, D. Steed (LFCC) 
Carex cephalophora Muhl. ex Willd. (oval-leaf sedge)

- Massanutten Mountain at Little Fort Recreation area along a jeep trail and stream south of campgrounds; moist stream side; Elev. 1500 ft.; 5/19/1977; R.C. Simpson (LFCC)

- Massanutten Mountain, Little Fort recreation area, along Powells mountain trail (to Edinburg gap) about $1 \mathrm{mi}$. South of the Little Fort camping area; Along moist edge of jeep trail; Elev. 1390.; 6/26/1977; R.C. Simpson, D. Steed, G. Evans (LFCC)

Carex communis Bailey (fibrousroot sedge)

- Approx 2 mi. N.W. of junction of \#675 and \#678 on \#675 on the Massanutten Mtn; rich sugar maple alluvial forest.; 5/4/1977; R.C. Simpson (LFCC)

- Approx. 2 mi. Northwest of junction of Rt 675 and \#678 on Rt 675 on the Massanutten Mt; Shale barren.; 5/4/1977; (LFCC)

$\diamond$ Carex crinita Lam. var. crinita (fringed sedge)

Carex debilis Michx. (white edge sedge)

- Massanutten Mt., Little Fort Recreation Area, along Powell's Mt. Trail (to Edinburg Gap) about 1 mi. south of the Little Fort Camping Area; Elev. 1390 ft.; 6/26/1977; R.C. Simpson, G. Evans, D. Steed (LFCC)

- West of Edinburg GWNF; Little Sluice Trail.; 6/27/1980; R.C. Simpson (LFCC)

Carex digitalis Willd. (slender woodland sedge)

- Massanutten Mountain Little Fort recreation area, along Powell's mountain trail (to Edinburg Gap) about 1 mi. South of the Little Fort camping area; Rick deciduous woods.; 6/26/1977; R.C.

Simpson, G. Evans (LFCC)

$\diamond$ Carex eburnea Boott (bristleleaf sedge)

$\diamond$ Carex echinata Murray ssp. echinata (star sedge)

$\diamond$ Carex flaccosperma Dewey (thinfruit sedge)

Carex frankii Kunth (Frank's sedge)

- Fisher's Hill dam, wet meadow.; 9/5/1975; R.C. Simpson (LFCC)

- $1 \mathrm{mi}$. South of Strasburg $40 \mathrm{ft}$. from the edge of the Strasburg reservoir; edge of woodland trail.; 7/26/1977; R.C. Simpson (LFCC)

$\diamond$ Carex glaucodea Tuck. ex Olney (blue sedge)

Carex gracilescens Steud. (slender looseflower sedge)

- Fort Valley Powell's Fort Camp bog where the electric line cuts across the wet lands; Open wet lands on meadow cut by deforestation along power lines.; 5/1/1977; R.C. Simpson (LFCC)

Carex gracillima Schw. (graceful sedge)

- Powell's Fort Bog.; 6/8/1980; R.C. Simpson (LFCC)

- $\quad$ Fort Valley; Powell's Fort Camp bog where the electric line cuts across the wetland.; 5/31/1977; R.C. Simpson (LFCC) 
Carex granularis Muhl. ex Willd. (limestone meadow sedge)

- $\quad$ On Rt. \#678 approx. 3 mi. north of Kings Crossing.; 6/8/1980; R.C. Simpson (LFCC)

- $\quad$ Cedar Creek; Panther Cave; Along the creek bank.; 7/9/1994; A. Fletcher (LFCC)

$\diamond$ Carex grisea Wahlenb. (inflated narrow-leaf sedge)

$\diamond$ Carex gynandra Schwein. (nodding sedge)

$\diamond$ Carex hirsutella Mack. (fuzzy wuzzy sedge)

$\diamond$ Carex hitchcockiana Dewey (Hitchcock's sedge)

Carex hystericina Muhl. ex Willd. (bottlebrush sedge)

- $\quad$ Approx. 2 miles North of Woodstock off Rt. 11 at Pugh's Run; over limes stone at stream edge.; 6/5/1977; R.C. Simpson, K.D. Ramsey (LFCC)

- $\quad$ Peter Mill Bog; Bog.; 6/16/1978; R.C. Simpson (LFCC)

Carex intumescens Rudge (greater bladder sedge)

- $\quad$ Little Fort V.; Bog; Altitude 1400.; 7/8/1965; L. Artz (JMUH)

- Massanutten Mountain Range (northern); Mudhole Bog.; 7/28/1978; B. Latham 45 (JMUH)

- Massanutten Mountain at Little Fort Recreation area along a jeep trail and stream south of campgrounds; Along edge of stream and sphagnaceans temporary pools; Locally common; Elev. 1500.; 5/19/1977; R.C. Simpson (LFCC)

- $\quad$ Powell's Fort Bog.; 6/8/1980; R.C. Simpson (LFCC)

Carex laevivaginata (Kük.) Mack. (smoothsheath sedge)

- Peter Mill Bog; Bog.; 6/16/1978; R.C. Simpson (LFCC)

- Open wet, Carex meadow.; 5/31/1977; R.C. Simpson (LFCC)

$\diamond$ Carex laxiculmis Schwein. var. laxiculmis (spreading sedge)

Carex laxiflora Lam. (broad looseflower sedge)

- Power line 300 yds E. of Rt 717; 4 mi. NE of Basye; moist/boggy near creek.; 5/21/1984; S. Arey 25 (JMUH)

- $\quad 11 / 2$ miles E. of Strasburg on the Reservoir Road; stream side in deciduous woods.; 4/29/1977; R.C. Simpson (LFCC)

- Approx. 6 miles west of Columbia Furnace at Laurel Run .2 miles west on public access road off Rt. 691; Mesic deciduous forest.; 6/5/1977; R.C. Simpson, K.D. Ramsey (LFCC)

Carex leptalea Wahlenb. (bristlystalked sedge)

- Little Fort Recreation Area, Peter Mill's Trail bog; bog.; 6/16/1978; R.C. Simpson, M. Krouse, J.P. (LFCC)

$\diamond$ Carex lupulina Willd. (hop sedge)

Carex lurida Wahlenb. (shallow sedge)

- Powell's Fort Bog.; 6/8/1980; R.C. Simpson (LFCC)

- $\quad$ Little Ft. Recreation Area.; 6/19/1975; R.C. Simpson (LFCC) 
$\diamond$ Carex muehlenbergii Schk. ex Willd. (Muhlenberg's sedge)

$\diamond$ Carex nigromarginata Schwein. var. nigromarginata (black edge sedge)

Carex normalis Mack. (greater straw sedge)

- 6 mi west of Columbia Furnace on Laurel Run at intersection of Rt 691 + Rt 717; Edge of seepage pond.; 6/5/1977; R. Simpson (LFCC)

$\diamond$ Carex oligocarpa Willd. (richwoods sedge)

Carex pensylvanica Lam. (Pennsylvania sedge)

- $\quad$ Rt 869 near Wolf Gap burn site; Burn site, wooded area.; 5/22/1979; R. Simpson (LFCC)

Carex platyphylla Carey (broadleaf sedge)

- Approx. 2 mi. N.W. of the jct. of Rt. \#675 and \#678 on \#675 on the Massanutten Mtn; rich sugar maple alluvial forest.; 5/4/1977; R.C. Simpson (LFCC)

$\diamond$ Carex prasina Wahlenb. (drooping sedge)

$\diamond$ Carex retroflexa Muhl. ex Willd. (reflexed sedge)

$\diamond$ Carex reznicekii Werier

Carex rosea Schk. ex Willd. (rosy sedge)

- 6 mi west of Columbia Furnace on Laurel Run at intersection of Rt $691+$ Rt 717; at edge of seepage pond.; 6/5/1977; R. Simpson (LFCC)

- Woodstock reservoir; Elev. 1290 feet.; 5/27/1980; R.C. Simpson, S. Gwyn (LFCC)

$\diamond$ Carex scabrata Schwein. (eastern rough sedge)

Carex scoparia Schk. ex Willd. (broom sedge)

- $\quad$ Rt. 685, 1.3 mi N from Rt. 675 NW of Edinburg; Elev. 850 ft.; 6/19/1977; G. Evans, K. Ramsey, R.C. Simpson (LFCC)

- $\quad$ West of Edinburg GWNF; Little Sluice Trail.; 6/27/1980; R.C. Simpson (LFCC)

$\diamond$ Carex seorsa Howe in Gordinier \& Howe (weak stellate sedge)

Carex shortiana Dewey (Short's sedge)

- 6 mi. West of Columbia Furnace on Laurel Run at intersection of Rt\#691 + Rt\#717; Edge of spring fed stream.; 6/5/1977; R. Simpson (LFCC)

Carex sparganioides Muhl. ex Willd. (bur-reed sedge)

- $\quad$ Route 6851.3 mile north from Route 675; large wet seepage pond in cow pasture; Elev. $850 \mathrm{ft}$; 6/19/1977; G. Evans, K. Ramsey (LFCC) 
Carex squarrosa L. (squarrose sedge)

- Devil's Backbone State Forest on trail in woods.; 5/10/2011; 39.04911,-78.43465; Domangue, B. Burkholder 179

- $\quad$ Fort Valley, Big Spring.; 7/17/1975; R. \& H. Simpson (LFCC)

- $\quad$ Fort Valley, 1/4 mile E. of Glass house.; 6/22/1980; R.C. Simpson (LFCC)

$\diamond$ Carex stipata Muhl. ex Willd. var. stipata (owlfruit sedge)

Carex striatula Michx. (lined sedge)

- Approx. 2 mi. N.W. of junction of \#675 and \#678 on \#675, on Massanutten Mtn; rich alluvial woods.; 5/4/1977; R.C. Simpson (LFCC)

Carex stricta Lam. in J. Lam., et al (upright sedge)

- Cedar Creek; Panther Cave; bank along creek, limestone cliffs.; 7/9/1994; A. Fletcher, (LFCC)

Carex styloflexa Buckley (bent sedge)

- $\quad 1$ 1/2 mi. East of Strasburg on the Reservoir Road; streamside in deciduous woods.; 4/29/1977;

R.C. Simpson (LFCC)

- Massanutten Mountain, Little Fort recreation area, along Powell's mountain trail (to Edinburg Gap) about 1 mi. South of the Little Fort Camping area; Along cool moist flood plain of Poters Mill Run.; 6/26/1977; R.C. Simpson, G. Evans (LFCC)

Carex swanii (Fernald) Mack. (Swan's sedge)

- Approx 6 miles West of Columbia Furnace on Laurel Run at the intersection of Rt. 691 and Rt. 717 .2 miles West on Rt. 691; Mesic cove forest.; 6/5/1977; R.C. Simpson, K.D. Ramsey (LFCC)

- West of Edinburg GWNF; Little Sluice Trail.; 6/27/1980; R.C. Simpson (LFCC)

$\diamond$ Carex tetanica Schkuhr (rigid sedge)

Carex torta Bott ex Tuck. (twisted sedge)

- $\quad$ Growing in small stream in public park near Lake Laura.; 4/11/2011; 38.80494,-78.78959; Domangue 83

Carex tribuloides Wahlenb. (blunt broom sedge)

- On Rt. \#678 approx. 3 mi. north of Kings Crossing.; 5/31/1977; R.C. Simpson (LFCC)

- Massanutten Mtn. Little Fort Recreation area, along Powell's Mtn. trail (to Edinburg Gap) about 1 mi. South of the Little Fort camping area; Along moist edge of Jeep trail.; 6/26/1977; R.C. Simpson, G. Evans (LFCC)

Carex umbellata Schk. ex Willd. (parasol sedge)

- North of Tom's brook on \#651 about 5 mi. west of \#11 (between \#81 and \#11); Open oak-hickory woods.; 5/4/1977; R.C. Simpson (LFCC)

Carex virescens Muhl. ex Willd. (ribbed sedge)

- Rt. \#675 Wolf Gap 1/2 mile E. of W. and Va. state line; campside next to trail, up trail to top of Big Shloss Mt.; G. Evans, (LFCC) 
Carex vulpinoidea Michx. (fox sedge)

- Massanutten Mountain, Little Fort recreation area along Powell's mountain trail (to Edinburg Gap) about 1 mi. South of the Little Fort camping area; Along moist Jeep trail; Elev. 1390.; 6/26/1977; G. Evans, D. Steed (LFCC)

- $\quad$ Little Ft. Recreation Area.; 6/19/1975; R.C. Simpson (LFCC)

Carex willdenowii Schk. ex Willd. (Willdenow's sedge)

- Fort Valley, 1/4 mile E. of Glass house.; 6/22/1980; R.C. Simpson (LFCC)

$\diamond$ Carex woodii Dewey (pretty sedge)

$\diamond$ Cyperus bipartitus Torr. (slender flatsedge)

$\diamond$ Cyperus diandrus Torr. (umbrella flatsedge)

Cyperus esculentus L. (yellow nutsedge)

- Valley View Orchard on Route 622 west of Strasburg; Orchard rows near pond; Elev. 800'.; 8/25/1978; B. Rinker (LFCC)

Cyperus flavescens L. (yellow flatsedge)

- Fisher's Hill at \#601 to 801 at Fisher's Hill Dam; on the dam's edge.; 9/17/1975; K.D. Ramsey (LFCC)

$\diamond$ Cyperus lancastriensis Porter ex Gray (manyflower flatsedge)

Cyperus lupulinus (Spreng.) Marcks (Great Plains flatsedge)

- In Ft. Valley on Rt \#678, 2 mi N of Detrick; grassy wetland on E side of road.; 9/20/1975; K.D. Ramsey (LFCC)

- Massanutten Mountain, Little Fort recreation area along Powell's mountain trail (to Edinburg gap) about $1 \mathrm{mi}$. South of the Little Fort camping trail; Along moist edge of jeep trail, Little Fort recreation area along Powell's mountain trail; Elev. 1390.; 6/26/1977; R.C. Simpson (LFCC)

$\diamond$ Cyperus odoratus L. var. odoratus (fragrant flatsedge)

Cyperus strigosus L. (strawcolored flatsedge)

- Rt. 730 roadside at forest border.; 7/29/2011; 38.71358,-78.58472; Domangue 482

- $\quad$ Mud Hole Gap; Elev. 1250 ft.; 9/19/1974; R.C. Simpson (LFCC)

- Massanutten Mountain, Mine Run Estates.; 9/23/1978; R.C. Simpson (LFCC)

Eleocharis engelmannii Steud. (Engelmann's spikerush)

- On Rt. \#678 approx. 3 mi. north of Kings Crossing.; 5/31/1977; R.C. Simpson (LFCC)

- $\quad$ Rt. 685, 1.3 mi N from Rt. 675 NW of Edinburg; Elev. 850 ft.; 6/19/1977; G. Evans, K. Ramsey, R.C. Simpson (LFCC)

Eleocharis erythropoda Steud. (bald spikerush)

- Cedar Creek; Panther Cave; wet area near creek.; 7/9/1994; A. Fletcher (LFCC)

- Approx. 2 miles North of Woodstock off US Rt. 11 at Pugh's Run; on small barren limestone islands in middle of stream.; 6/5/1977; R.C. Simpson, K.D. Ramsey (LFCC) 
Eleocharis obtusa (Willd.) Schult. (blunt spikerush)

- Tomahawk Pond Campground, on the edge of pond.; 8/8/2011; 38.75883,-78.84042; Domangue 529

- $\quad$ Little Fort; Bog; Altitude 1500; 7/29/1965; L. Artz (JMUH)

- $\quad$ Little Ft. Recreation Area.; 6/19/1975; R.C. Simpson (LFCC)

- Massanutten Mountain, Mine Run Estates.; 9/23/1978; R.C. Simpson (LFCC)

$\diamond$ Eleocharis smallii Britton (common spikerush)

Eleocharis tenuis (Willd.) Schult. (slender spikerush)

- Vances Cove; Bog; Altitude 1500.; 7/14/1965; L. Artz (JMUH)

- $\quad$ Little fort roadside 1 mi. S. of rec. area.; 5/22/1976; C.R. Hupp (LFCC)

- On Rt. \#678 approx. 3 mi. north of Kings Crossing.; 5/31/1977; R.C. Simpson (LFCC)

Eriophorum virginicum $\mathrm{L}$. (tawny cottongrass)

- $\quad$ Little Fort V.; Muskeg; Altitude 1400.; 7/29/1965; L. Artz (JMUH)

$\diamond$ Fimbristylis autumnalis (L.) R. \& S. (slender fimbry)

$\diamond$ Rhynchospora capitellata (Michx.) Vahl (brownish beaksedge)

$\diamond$ Rhynchospora glomerata (L.) Vahl (clustered beaksedge)

$\diamond$ Schoenoplectus pungens (Vahl) Palla (common threesquare)

+ Schoenoplectus purshianus (Fernald) Strong (weakstalk bulrush)

- $\quad$ Tomahawk Pond Campground, growing in pond.; 9/22/2011; 38.75877,-78.84037; Domangue 635

Schoenoplectus tabernaemontani (Gmelin) Palla (softstem bulrush)

- $\quad$ Fisher's Hill Dam; wet meadow.; 9/5/1975; R. Simpson (LFCC)

- 6 mi west of Columbia Furnace on Laurel Run at intersection of Rt \#691 + Rt \#717; edge of spring fed seepage area.; 6/5/1977; R. Simpson (LFCC)

Scirpus atrovirens Willd. (green bulrush)

- $\quad$ On Rt \#678 app. 3 mi North of Kings Crossing.; 5/31/1977; R.C. Simpson (LFCC)

- Massanutten Mountain, Little Fort recreation area along Powell's mountain trail (to Edinburg Gap) about 1 mi. South of the Little Fort camping area; Along moist Jeep trail; Elev. 1390.; 6/26/1979; G. Evans, D. Steed (LFCC)

Scirpus cyperinus (L.) Kunth (woolgrass)

- Devil's Backbone State Forest in grassy clearing.; 6/8/2011; 39.04882,-78.43182; Domangue 319

- Ft. Valley at Powell's Ft. Camp on Rt \#772; grassy telephone cut on E roadside.; 9/19/1975; K.D. Ramsey (LFCC)

$\diamond$ Scirpus georgianus Harper (Georgia bulrush)

$\diamond$ Scirpus hattorianus Makino (mosquito bulrush) 
Scirpus pendulus Muhl. (rufous bulrush)

- Devil's Backbone State Forest, beside dirt road.; 7/20/2011; 39.04836,-78.43542; Domangue, C.K. McMullen 473

- Fort Valley, growing in open, grassy meadow.; 6/24/2011; 38.88321,-78.37984; Domangue, J. Courtwright 383

- Approx. 2 miles North of Woodstock off US Rt. 11 at Pugh's Run; Over limestone at edge of stream.; 6/5/1977; R.C. Simpson, K.D. Ramsey (LFCC)

Scirpus polyphyllus Vahl (leafy bulrush)

- $\quad$ Tomahawk Pond Campground, in boggy area on the edge of pond.; 8/8/2011; 38.75896, -78.84065 ; Domangue 530

$\diamond$ Scleria triglomerata Michx. (whip nutrush)

Dioscoreaceae

Dioscorea polystachya Turczaninow (Chinese yam) *

- $\quad$ Rt. 672 roadside near N. Fork Shenandoah River.; 7/12/2011; 38.84419,-78.53230; Domangue 427

Dioscorea villosa $\mathrm{L}$. (wild yam)

- Elizabeth Furnace Recreation Area. Twining plant in riparian zone near Passage Creek.; 6/24/2011; 38.92887,-78.33064; Domangue, J. Courtwright 376

- Moist woods; Rt. 678 Fort Valley.; 5/21/1986; (JMUH)

- $\quad$ FR 274 West.; 7/22/1981; D.E. Miller 1 (JMUH)

- Rt \#691, Bear Oak Prescribed Burn, near Va.-W.Va. state line, along Laurel Run trail; Unburned area; Elev. 3005 feet.; 5/22/1981; S. Collins (LFCC)

- Woodstock; George Washington National Forest.; 10/3/1979; K. Motiska, K. Halstead (LFCC)

Hydrocharitaceae

Elodea canadensis Michx. (Canadian waterweed)

- $\quad$ Passage Creek; Water; Altitude 800.; 7/22/1965; L. Artz (JMUH)

- $\quad$ NE end Edinburg. Jct. Stoney Creek and Shen. River. Rt. 675; Elev. 850 ft.; 6/19/1977;

K. Ramsey, G. Evans, R.C. Simpson (LFCC)

$\diamond$ Elodea nuttallii (Planch.) St. John (western waterweed)

Iridaceae

$\diamond$ Belamcanda chinensis (L.) DC. (blackberry lily) * 
Iris verna $\mathrm{L}$. (dwarf violet iris)

- $\quad$ Rt. 730 roadside, at forest border.; 4/19/2012; 38.72973,-78.56576; Domangue 674

- Power line 300 yds. E. of Rt. 717; 4 mi. NE. of Basye; shady, edge of woods.; 5/15/1984; S. Arey 12 (JMUH)

- $\quad$ Rt \#691, Bear Oak Prescribed Burn, near Va.-W.Va. state line; along roadside of burned area; flower - purple; Elev. 3005 feet.; 5/3/1981; S.M. Collins (LFCC)

- Approx. 2 mi. N.W. of junction of \#675 and \#678 on \#675 on the Massanutten; edge of rich woods.; 5/4/1977; R.C. Simpson (LFCC)

Sisyrinchium angustifolium Mill. (narrowleaf blue-eyed grass)

- Camp Roosevelt at small clearing in forest.; 5/25/2011; 38.72945,-78.51690; Domangue 255

- Woodstock; Turn off Rt. 11 and go 1 1/4 miles East on Lakeview Road and take left on old dirt road and go 3/4 miles; River bottom meadow.; 7/5/1982; J. Nutter, 1 (JMUH)

- $\quad$ On Rt. \#678 approx. 3 mi. north of Kings Crossing.; 5/31/1977; R.C. Simpson (LFCC)

- Powell's Fort Bog.; 6/8/1980; R.C. Simpson (LFCC)

Sisyrinchium mucronatum Michx. (needletip blue-eyed grass)

- Tomahawk Pond Campground, in clearing next to pond. Clay soil.; 5/10/2011; 38.75791, -78.84087; Domangue, B. Burkholder 161

- Route 6781 mile north of intersection with route 67510 miles west of Edinburg; Roadside, open.; 5/16/1982; L. Michael Hill 9 (BDWR)

- $\quad$ Little Sluice Mountain trail; flower blue.; 5/6/1980; R.C. Simpson (LFCC)

- Fisher's Hill Dam; Fisher's Hill, 2 mi. south of Strasburg, growing on a rocky south facing slope.; 4/30/1975; K. Ramsey (LFCC)

Juncaceae

Juncus acuminatus Michx. (tapertip rush)

- Tomahawk Pond Campground, on the edge of pond.; 8/8/2011; 38.75883,-78.84042; Domangue 528

- $\quad$ On Rt. \#678 approx. 3 mi. N. of Kings Crossing; wet meadow along intermitent stream at base of shale barrens.; 5/3/1977; R.C. Simpson (LFCC)

- Rt. 685; 1.3 mi. N from Rt. 675 NW of Edinburg; Elev. 850 ft.; 6/19/1977; G. Evans, K. Ramsey, R.C. Simpson (LFCC)

$\diamond$ Juncus bufonius L. (toad rush)

+ Juncus dichotomus Ell. (forked rush)

- Devil's Backbone State Forest in grassy clearing.; 6/8/2011; 39.04882,-78.43182; Domangue 317

Juncus dudleyi Wieg. (Dudley's rush)

- $\quad$ Approx. 2 miles North of Woodstock off US Rt. 11 at Pugh's Run; on small barren limestone islands in middle of stream.; 6/5/1977; R.C. Simpson, K.D. Ramsey (LFCC)

- Rt. 6851.3 mi. N. from Rt. 675; (NW Edinburg); Elev. 850 ft.; 6/19/1977; G. Evans, K. Ramsey, R.C. Simpson (LFCC) 
Juncus effusus L. (common rush)

- $\quad$ Devil's Backbone State Forest in grassy clearing.; 6/8/2011; 39.04882,-78.43182; Domangue 318

- $\quad$ Elizabeth Furnace Recreation Area near Passage Creek.; 5/25/2011; 38.92837,-78.33027; Domangue 263

- $\quad$ Little Fort V.; Bog; Altitude 1400.; 7/8/1965; L. Artz (JMUH)

- $\quad$ George Washington National Forest, 1 mi. south of Elizabeth Furnace.; 5/1/1978; R.C. Simpson (LFCC)

- $\quad$ Fisher's Hill dam, wet meadow.; 9/5/1975; R.C. Simpson (LFCC)

Juncus marginatus Rostk. (grassleaf rush)

- Devil's Backbone State Forest, in grassy clearing in woods.; 7/20/2011; 39.04879,-78.43174; Domangue, C.K. McMullen 468

$\diamond$ Juncus nodosus L. (knotted rush)

$\diamond$ Juncus scirpoides Lam. (needlepod rush)

Juncus secundus Beauv. ex Poir. (lopsided rush)

- $\quad$ Little Ft. Recreation Area.; 6/17/1975; R.C. Simpson (LFCC)

$\diamond$ Juncus subcaudatus (Engelm.) Coville \& Blake var. subcaudatus (woodland rush)

Juncus tenuis Willd. (poverty rush)

- Devil's Backbone State Forest. Growing beside forest road.; 6/8/2011; 39.04760,-78.43163; Domangue 316

- $\quad$ West of Edinburg GWNF; Little Sluice Trail.; 6/27/1980; R.C. Simpson (LFCC)

- On Rt. \#678 approx. 3 mi. north of Kings Crossing.; 5/31/1977; R.C. Simpson (LFCC)

Juncus torreyi Coville (Torrey's rush)

- $\quad$ Fisher's Hill dam, wet meadow.; 9/5/1975; R.C. Simpson (LFCC)

$\diamond$ Luzula acuminata Raf. (hairy woodrush)

Luzula bulbosa (Wood) Smyth \& Smyth (bulbous woodrush)

- Intermittent pond; at Wolf Gap on Rt \#691 1/2 mi. E. of West Va. Line.; 6/9/1975; R.C. Simpson (LFCC)

Luzula echinata (Small) F.J. Herm. (hedgehog woodrush)

- Fort Valley Powell's Fort Camp bog where the electric line cuts across the wet lands; Open wet lands, cleared by deforestation along the power line.; 5/31/1977; R.C. Simpson (LFCC)

- North of Tom's brook on \#651 about 5 mi. west of \#11 (between \#81 and \#11); Open oak-hickory woods.; 5/4/1977; R.C. Simpson (LFCC)

Luzula multiflora (Ehrh.) Lej. (common woodrush)

- Devil's Backbone State Forest on trail in woods.; 5/10/2011; 39.04850,-78.43546; Domangue, B. Burkholder 180

- $\quad$ Fort Valley, on edge of wooded area and grassy field.; 4/26/2011; 38.88359,-78.37228; Domangue, M. Lantz 116

- West of Edinburg GWNF; Little Sluice Trail.; 6/27/1980; R.C. Simpson (LFCC) 
Lemnaceae

Lemna minor L. (common duckweed)

- $\quad$ Laurel Run Road; 1/2 west of Rt 691 on north side of road; Bog area; Elev. 1,300 feet; 7/7/1980;

S. Gwyn, R.C. Simpson (LFCC)

- $\quad$ Fisher's Hill, 2 mi. south of Strasburg, growing in the water (on top of the water).; 5/2/1975;

K. Ramsey (LFCC)

$\diamond$ Lemna perpusilla Torr. (minute duckweed)

Lemna valdiviana Phil. (valdivia duckweed)

- $\quad$ In cow pasture, floating on the surface of small stream.; 8/8/2011;38.79440,-78.66000;

Domangue 542

- $\quad$ Pond at farm near Detrick.; 8/10/1971; C.A. Helsley (JMUH)

Spirodela polyrrhiza (L.) Schleid. (common duckmeat)

- $\quad$ Approx. 6 miles West of Columbia Furnace on Laurel Run at the intersection of Rt. $691+$ Rt. 717 in seepage ponds .2 mi. West on Rt. 691; clear slow flowing water in seepage ponds and dammed up ponds; Elev. 1500 ft.; 6/5/1977; R. Simpson, K. Ramsey (LFCC)

- $\quad$ Valley View Orchard west of Strasburg on Route 622; Open bond in apple orchard; Elev. 800'.; 8/22/1978; B. Rinker (LFCC)

\section{Liliaceae}

Allium canadense L. (meadow garlic)

- $\quad 3.0$ mi. NE of Lebanon Church on Route 628. Floodplain of stream; Edible parts: Bulbs and leaves eaten raw or boiled. Bulbs also used as a seasoning.; 5/21/1988; Cason, Gordon 14 (JMUH)

- $\quad 3.0$ miles NE of Lebanon Church on Rt 628.; 5/21/1988; Chandler 8 (JMUH)

- $\quad$ Peter Mill Trail; Little Fort Recreation.; 6/16/1978; R.C. Simpson (LFCC)

Allium cernuum Roth (nodding onion)

- $\quad$ Rt. 776 roadside, near intersection with Rt. 678.; 7/29/2011; 38.79728,-78.46316; Domangue 498

- $\quad$ FR 274 West side.; 7/8/1981; D.E. Miller 42 (JMUH)

- $\quad$ Elizabeth Furnace; shale barrens.; 7/13/1979; R.C. Simpson (LFCC)

- $\quad$ Rt. \#678, near St. David's Church sign.; 7/13/1975; R.C. Simpson (LFCC)

$\diamond$ Allium tricoccum Ait. (ramp)

Allium vineale $\mathrm{L}$. (wild garlic) *

- $\quad$ Elizabeth Furnace Recreation Area, on the edge of lawn.; 6/24/2011; 38.92771,-78.33057; Domangue, J. Courtwright 374

- $\quad$ Rt. 620 roadside, adjacent open field.; 6/15/2011; 38.69464,-78.64809; Domangue 334

- Massanutten Mountain .5 mi. East of the Little Fort recreation area; moist along roadside.; 6/26/1977; R.C. Simpson, D. Steed (LFCC)

Amianthium muscitoxicum (Walt.) Gray (flypoison)

- $\quad$ Little Passage Creek; Dry Woods; Altitude 950 ft.; 8/6/1964; L. Artz (JMUH) 
Asparagus officinalis $\mathrm{L}$. (garden asparagus) *

- Cedar Grove Church. Edge of parking lot in tall grasses. $1 \mathrm{~m}$ tall.; 5/18/2011; 38.69433, -78.64742; Domangue 210

- Found along side a deserted garden approx. 10 miles North of Woodstock on Rt. 707. Plant was growing profusely within the confines of the garden and along the fence row.; 6/30/1977; T. Burke 12 (JMUH)

- $\quad$ Open wasteland; South of Rt. 55 - 4 mi. west of Lebanon Church.; 5/13/1975; M. Dyson-Cobb (LFCC)

- $\quad$ Rt 6231 mile South of Zepp (Maurertown) Rd.; Elev. 1100 ft.; 4/29/1980; S. Gwyn (LFCC)

Chamaelirium luteum (L.) Gray (fairywand)

- $\quad$ Powell's Fort Area; Mud hole Gap; 6/24/1978; R.C. Simpson (LFCC)

$\diamond$ Convallaria majalis L. (European lily of the valley) *

Erythronium americanum Ker-Gawl. (dogtooth violet)

- $\quad$ Fort Valley, growing in weedy area beside Passage Creek.; 4/26/2011; 38.88425,-78.37561;

Domangue, M. Lantz 131

- $\quad$ Strasburg, Frontage Road.; 4/13/1977; R.C. Simpson (LFCC)

- Rt. 55 west, 1 mile east of the Junc. of the Fredrick Co. and Shenandoah line, along road pull-off on the south side of a steep hill leading to Turky Run Stream; Steep hillside leading down to a small stream, wooded area.; 4/22/1989; E.A. Moore (LFCC)

Erythronium umbilicatum Parks \& Hardin (dimpled troutlily)

- On North bank of Stoney Creek about 150-200 yards on the South side of Stoney Creek Rd., 1-1.5 miles East of the Rt. 42 junction (Larkin's Grocery), approximately 1/4 miles downstream from swinging bridge.; 4/13/2001; I. Poplar-Jeffers 6 (JMUH)

- $\quad$ Near Wolf Gap; fl. Yellow.; 4/7/1977; C.R. Hupp, R.C. Simpson (LFCC)

Hemerocallis fulva (L.) L. (orange daylily) *

- $\quad$ Rt. 620 roadside, along old fencerow.; 6/15/2011; 38.69464,-78.64809; Domangue 335

- $\quad$ Little Fort Rd; Road Side; Elev. 2,000 feet.; 6/29/1980; S. Gwyn (LFCC)

Hypoxis hirsuta (L.) Coville (common goldstar)

- Conicville. On shady dirt path in woods.; 5/20/2011; 38.83255,-78.67323; Domangue 240

- $\quad$ Northern Massanutten Mountains; Mudhole Bog.; 7/5/1978; J. Huffman, B. Latham, D. Stoltzfus 20 (JMUH)

- Mudhole Bog by Little Passage Creek in Little Fort Valley. Growing in a boggy area along a stream.; 6/28/1972; BIO 68518 (JMUH)

- Ft. Valley, Little; Fort Recreation area.; 6/19/1975; R.C. Simpson (LFCC)

- Bear Wallow Trail, George Washington National Forest; open woods.; 8/2/1978; S.M. Collins (LFCC)

Lilium canadense L. (Canada lily)

- $\quad$ Kings Crossing on the Massanutten Mt.; 6/26/1975; R.C. Simpson (LFCC)

$\diamond$ Lilium philadelphicum L. (wood lily)

$\diamond$ Maianthemum canadense Desf. (Canada mayflower) 
Maianthemum racemosum (L.) Link (feathery false lily of the valley)

- $\quad$ FR 374 roadside bank at forest border. $1 \mathrm{~m}$ tall. Dark, silty soil.; 5/30/2012; 38.78378, -78.53439; Domangue 706

- $\quad$ Rt. 730 roadside at the edge of woods.; 8/23/2011; 38.71286,-78.58624; Domangue 568

- $\quad$ Fort Valley Rt 675.; 5/21/1986; (JMUH)

- 2 miles west of King's Crossing (junction of 675 and 678) on Rte 675. Roadside.; 5/17/1988; Cowherd, Francisco, Thomas 26 (JMUH)

- $\quad$ Powell's Fort Camp; Bog.; 10/8/1979; T. Leight, C. Hupp, R.C. Simpson (LFCC)

- Low, open woods; $1 / 4$ mi N of Strasburg, behind F.R. Hupp property.; 5/15/1975; C.R. Hupp (LFCC)

Medeola virginiana $\mathrm{L}$. (Indian cucumber)

- Rt \#691, Bear Oak Prescribed Burn, near Va.-W.Va. state line; 300 yards north on Laurel Run Trail off Rt \#691; Elev. 3005 feet.; 5/24/1981; S. Collins (LFCC)

- $\quad 2$ mi W of Columbia Furnace on Rt. \#675; Flowers: yellow.; 6/9/1975; R.C. Simpson (LFCC)

Muscari botryoides (L.) Mill. (common grape hyacinth) *

- $\quad$ Edinburg, at the end of Shenandoah Avenue. Growing in a vacant grassy lot.; 4/18/2011; 38.82873,-78.56366; Domangue, J. Courtwright 106

Muscari neglectum Guss. ex Ten. (starch grape hyacinth) *

- Clearing in wooded area near small cabin.; 5/11/2011; 38.94997,-78.52692; Domangue, C.K. McMullen 198

- $\quad$ Fort Valley.; 4/25/1980; T. McCracken (LFCC)

- $\quad$ Fort Valley.; 4/9/1977; R.C. Simpson (LFCC)

Ornithogalum umbellatum L. (sleepydick) *

- $\quad$ Fort Valley, in grassy field along roadside.; 5/4/2011; 38.88425,-78.37693; Domangue 144

- $\quad$ Creek which runs through picnic area, Elizabeth Furnace; Woods.; 5/16/1982; L. Michael Hill 7 (BDWR)

- George Washington National Forest, Elizabeth Furnace; Deciduous forest next to river; Elev. 800 ft.; 5/22/1978; R.F. Whiting (LFCC)

Polygonatum biflorum (Walt.) Ell. (smooth Solomon's seal)

- Clearing in wooded area near small cabin.; 5/11/2011; 38.94997,-78.52692; Domangue, C.K. McMullen 188

- Mt. Jackson; shale mountain.; 5/1983; J. Kauffman (HAVI)

- $\quad$ Along Route 675, 3.6 mi. east of Edinburg. Shale barrens; Edible Parts: Young shoots, roots.; 5/17/1988; Gordon, Cason 1 (JMUH)

- 2 miles west of King's Crossing (junction of 675 and 678) on Rte 675. Roadside.; 5/17/1988; Cowherd, Francisco, Thomas 25 (JMUH)

- $\quad$ Rt. 6753.6 miles; Edinburg Shale Barrens.; 5/17/1988; Burnette, Mayes 11 (JMUH)

- 1 mile E of Liberty Furnace at junction of Rt. 717 and 690; Shale barren.; 9/7/1975; R.C. Simpson (LFCC)

- Rt \#691, Bear Oak Prescribed Burn, near Va.-W.Va. state line; along roadbank of burned area; Elev. 3005 feet.; 5/21/1981; S. Collins (LFCC)

$\diamond$ Polygonatum pubescens (Willd.) Pursh (hairy Solomon's seal) 
$\diamond$ Stenanthium gramineum (Ker-Gawl.) Morong (eastern featherbells)

$\diamond$ Trillium erectum $\mathrm{L}$. (red trillium)

$\diamond$ Trillium grandiflorum (Michx.) Salisb. (white trillium)

Trillium sessile L. (toadshade)

- Passage Creek; Woods; Altitude 900 ft.; 5/16/1964; L. Artz (JMUH)

- $\quad$ East of New Market on Clicks Lane at Smith Creek; Rich, Moist Roadbank.; 5/16/1984;

W. Heeb, C. Gunter 106 (JMUH)

- River bed bank; 2 1/4 mi. SE of Strasburg; Palmers River Bank on Shenandoah River; Flowers: maroon.; 4/13/1975; R.C. Simpson (LFCC)

Uvularia perfoliata $\mathrm{L}$. (perfoliate bellwort)

- $\quad$ North of Tom's brook on \#651 about 5 mi. west of \#11 (between \#11 and \#81); open wood land.; 5/4/1977; R.C. Simpson (LFCC)

Uvularia puberula Michx. (mountain bellwort)

- Moist woods; 2 mi S of Strasburg on Rt 748.; 5/1/1975; C.R. Hupp (LFCC)

- Rt \#691, Bear Oak Prescribed Burn, near Va.-W.Va. state line; Burned area; Elev. 3005 feet.; 5/24/1981; S. Collins (LFCC)

$\diamond$ Uvularia sessilifolia $\mathrm{L}$. (sessileleaf bellwort)

Veratrum parviflorum Michx. (Appalachian bunchflower)

- $\quad$ Mudhole Bog Powell's Fort; Swamp area.; 6/22/1977; R.F. Pugh, Jr. 12 (JMUH)

- West of Edinburg GWNF; Little Sluice Trail.; 6/27/1980; R.C. Simpson (LFCC)

Veratrum virginicum (L.) Aiton (Virginia bunchflower)

- Mudhole Bog in Little Fort Valley Northern Massanutten Mt. range; Mostly vegetative, few flowering plants in the population. Sphagnous conditions.; 7/25/1973; N.L. Bodkin (JMUH)

- $\quad$ Little Fort Valley, Mud Hole Bog; Growing in sphagnum bog. Only a few plants in flower.; 8/11/2000; C.K. McMullen, N.L. Bodkin 790 (JMUH)

- Northern Massanutten Mountains; Mudhole Bog.; 7/5/1978; J. Huffman, B. Latham, D. Stoltzfus 15 (JMUH)

- $\quad$ Little Fort V.; Bog; Altitude 1400.; 7/29/1965; L. Artz (JMUH)

- Mudhole Bog Little Fort Valley, Northern Massanutten Mt. range; Growing in a sphagnous boglike area under a deciduous canopy; Plant very large, $16.25 \mathrm{dm}$ tall bulb to tip of the panicle.

Lower leaf 62 x 3.17 cm.; 7/27/1977; N.L. Bodkin (JMUH)

- Mudhole Bog, Little Fort Valley Northern Massanutten Mt. range; Small population of approximately 100 plants with few flowering ones growing in a sphagnous bog.; 7/28/1973; N.L. Bodkin (JMUH)

- Mudhole Bog, Little Fort Valley; Growing in a Sphagnum Bog, very few plants in the population flowering.; 7/26/1975; N.L. Bodkin (JMUH)

- Mudhole Bog in Little Fort Valley Northern Massanutten Mt. range; Approximately 160 plants in the population, only 16 of which were flowering. Sphagnous conditions.; 7/10/1972; N.L. Bodkin (JMUH) 
Veratrum viride Aiton (green false hellebore)

- $\quad$ Little Sluice Mountain Trail.; 5/6/1980; R.C. Simpson (LFCC)

$\diamond$ Xerophyllum asphodeloides (L.) Nutt. (eastern turkeybeard)

Najadaceae

$\diamond$ Najas guadalupensis (Spreng.) Magnus ssp. guadalupensis (southern waternymph)

Orchidaceae

$\diamond$ Aplectrum hyemale (Muhl. ex Willd.) Torr. (Adam and Eve)

Calopogon tuberosus (L.) B.S.P. (tuberous grasspink)

- $\quad$ Fort Valley; Peter's Mill Bog.; 6/24/1980; R.C. Simpson (LFCC)

$\diamond$ Corallorhiza maculata (Raf.) Raf. var. maculata (summer coralroot)

Corallorhiza odontorhiza (Willd.) Nuttall (autumn coralroot)

- $\quad$ Northern end of Fort Valley, $150 \mathrm{ft} \mathrm{N}$ of Signal Knob at trail parking area on Rt. 678.; 8/12/1975;

R.C. Simpson (LFCC)

- 1.2 miles south of Orkney Springs at Seven Springs; cove forest.; 9/9/1979; R.C. Simpson (LFCC)

Cypripedium acaule Ait. (moccasin flower)

- Fort Valley, on wooded hillside. Growing in established population.; 5/4/2011; 38.88503, -78.37359; Domangue 140

- Ft. Valley on Rt \#678 in Detrick; east on Rt 758 to Milford Gap; Pine woods 100' from a mountain stream.; Flower - Light Pink.; 5/4/1976; K.D. Ramsey (LFCC)

$\diamond$ Cypripedium parviflorum Salisb. (lesser yellow lady's slipper)

$\diamond$ Cypripedium reginae Walter (showy lady's slipper)

Galearis spectabilis (L.) Raf. (showy orchid)

- $\quad$ Laurel Run Rd.; Pink flowers; Elev. 1100 ft.; 5/13/1980; S. Gwyn (LFCC)

Goodyera pubescens (Willd.) R. Br. ex Aiton f. (downy rattlesnake plantain)

- Conicville, beside dirt trail in woods.; 8/2/2011;38.83258,-78.67318; Domangue 520

- Path to Powells bog, Powells Fort.; 7/25/1976; C.R. Hupp (LFCC)

- Rt. 675; George Washington National Forest; Lyon's Tale Trail.; 10/7/1979; J. Evans (LFCC)

Hexalectris spicata (Walter) Barnh. (spiked crested coralroot)

- $\quad 2$ mi. N. of Elizabeth Furnace; on top of wooded shale cliffs.; 8/14/1980; R.C. Simpson (LFCC) 
Isotria verticillata (Muhl. ex Willd.) Raf. (large whorled pogonia)

- $\quad$ Beetle Run; Open Hardwoods; Elev. 1500 ft.; 5/5/1980; M. Willeford, M. Hughs-Crabtree (LFCC)

- $\quad$ Approx. 1 mi west of junction Rt \#616 and Rt \#675 on Rt \#616 on south side of road approx. 5 mi; .25 mi downstream of spring on west bank; spring and seepage area, Amer Chestnut Oaks, Huckleberries, Dogwoods; Elev. 1400 ft.; 5/25/1978; R.C. Simpson, M. Krouse, J. Brumback (LFCC)

Liparis liliifolia (L.) L.C.Rich. ex Ker-Gawl. (brown widelip orchid)

- $\quad$ Laurel Run Road 1/2 mile west of Rt 691 on North side of road; Bog area; Elev. 1,300 feet.; 7/7/1980; R.C. Simpson, S. Gwyn (LFCC)

- Powells Fort Road. 1/2 mile from Rt 678; Woods.; 9/27/1975; G. Beaty (LFCC)

Liparis loeselii (L.) L.C.Rich. (yellow widelip orchid)

- $\quad$ Little Fort; Muskeg (Bog); Altitude 1400.; 8/18/1965; L. Artz (JMUH)

- Approx. 6 miles West of Columbia Furnace on Laurel Run at the intersection of Rt. 691 and Rt. 717.2 miles West on Rt. 691 (in seepage ponds); seepage area at edge of pond in open and partial shade.; 6/5/1977; R.C. Simpson, K.D. Ramsey (LFCC)

$\diamond$ Malaxis unifolia Michx. (green adder's-mouth orchid)

Platanthera ciliaris (L.) Lindl. (yellow fringed orchid)

- Laurel Run Road 1/2 mile west of Rt 691 on South side of road; Bog area; Elev. 1900 feet; 8/3/1980; S. Gwyn (LFCC)

Platanthera clavellata (Michx.) Luer (small green wood orchid)

- $\quad$ Little Passage Cr.; Dry woods; Altitude 1000 ft.; 8/6/1964; L. Artz (JMUH)

- $\quad$ Laurel Run Road 1/2 mile west of Rt 691 on South side of road; Bog area; Elev. 1,900 feet.; 8/3/1980; S. Gwyn (LFCC)

Platanthera lacera (Michx.) G.Don (green fringed orchid)

- $\quad$ Little Fort; Bog; Altitude 1400.; 7/8/1965; L. Artz (JMUH)

- $\quad$ Northern Massanutten Mountains; Mudhole Bog.; 7/5/1978; J. Huffman, B. Latham, D. Stoltzfus 24 (JMUH)

Platanthera psycodes (L.) Lindl. (lesser purple fringed orchid)

- $\quad$ Little Fort V.; Muskeg (Bog); Altitude 1400 ft.; 7/29/1965; L. Artz (JMUH)

$\diamond$ Pogonia ophioglossoides (L.) Ker-Gawl. (snakemouth orchid)

Spiranthes cernua (L.) Rich. (nodding lady's tresses)

- Powell's Fort Recreation Camp; fls. Creamy yellow.; 9/23/1978; R.C. Simpson (LFCC)

Spiranthes lacera (Raf.) Raf. (northern slender lady's tresses)

- $\quad$ Tomahawk Pond Campground in clearing in woods.; 9/8/2011; 38.75816,-78.84170; Domangue 603 
Spiranthes ochroleuca (Rydb.) Rydb. (yellow nodding lady's tresses)

- Laurel Run Road 1/2 mile west of Rt 691 on North side of road; Bog area; Elev. 1,900 feet.; 9/30/1980; S. Gwyn (LFCC)

- 1 mile South of Powells Fort Organization Camp; wooded roadside edge; flower yellowish-white.; 9/19/1975; Simpson, Hunt (LFCC)

Poaceae

Agrostis gigantea Roth (redtop) *

- Fort Valley, growing in open, grassy meadow.; 6/24/2011; 38.88321,-78.37984; Domangue, J. Courtwright 382

$\diamond$ Agrostis hyemalis (Walt.) B.S.P. (winter bentgrass)

Agrostis perennans (Walt.) Tuck. (upland bentgrass)

- $\quad$ Prescribed burn; Elev. 3000 ft.; 9/5/1980; R.C. Simpson (LFCC)

$\diamond$ Andropogon gerardii Vitman (big bluestem)

Andropogon virginicus L. (broomsedge bluestem)

- Growing in grassy field off rt. 711.; 10/27/2011;38.79589,-78.66165; Domangue 657

- Hockman Homestead 1 mile north of Edinburg U.S. Rt. 1125 feet west of dwelling; lawn.; 9/30/1975; G. Beaty (LFCC)

- Hockman Homestead 1 mile north of Edinburg U.S. Rt 11. 50 feet N.N.W. of dwelling; lawn.; 10/30/1975; G. Beaty (LFCC)

Anthoxanthum odoratum L. (sweet vernalgrass) *

- $\quad$ Elizabeth Furnace Recreation Area, growing in lawn near Passage Creek.; 4/19/2012; 38.92847, -78.33035; Domangue 680

- $\quad$ Elizabeth Furnace Recreation Area on the edge of lawn and weedy area near Passage Creek.; 5/4/2011; 38.92770,-78.33052; Domangue 151

- Massanutten Moutain, Little Fort recreation Area along Powell's Mountain trail; Along moist edge of Jeep trail.; 6/26/1977; G. Evans, D. Steed (LFCC)

$\diamond$ Aristida dichotoma Michx. var. dichotoma (churchmouse threeawn)

$\diamond$ Aristida oligantha Michx. (prairie threeawn)

Arrhenatherum elatius (L.) J. \& K. Presl (tall oatgrass) *

- $\quad$ Little Fort Recreation trail.; 6/13/1980; R.C. Simpson (LFCC)

- Hockman Homestead 1 mi north of Edinburg US Rt \#11 on east side of road; Elev. $850 \mathrm{ft}$; 6/20/1977; G. Evans (LFCC)

Arthraxon hispidus (Thunb.) Makino (small carpgrass) *

- $\quad$ Bowers Ln. roadside next to cultivated field.; 9/8/2011; 38.86383,-78.72741; Domangue 610

$\diamond$ Arundinaria gigantea (Walt.) Muhl. (giant cane) 
Avena sativa $\mathrm{L}$. (common oat) *

- yard; 1 mi. N. of Edinburg Rt 11, Hockman Homestead 100 ft. E. of dwelling.; 10/4/1975; W. Bridwell (LFCC)

- Hockman Homestead (Chequers) US Rt. 11 on east side of road 1 mi. north of Edinburg; lawn may have been imported through bird seed.; 7/9/1977; G. Evans (LFCC)

Bouteloua curtipendula (Michx.) Torr. (sideoats grama)

- Rt. 620 roadside, across from Cedar Grove Church.; 7/12/2011; 38.69439,-78.64739; Domangue 421

$\diamond$ Brachyelytrum aristosum (Michx.) Trel. in Banner \& Coville (northern shorthusk)

$\diamond$ Brachyelytrum erectum (Schreb. ex Spreng.) Beauv. (bearded shorthusk)

Brickellia eupatorioides (L.) Shinners (false boneset)

- $\quad 2.5$ miles west of Columbia Furnace junction of Rt. \#717 and \#675; Shale Barrens.; 9/7/1975;

R.C. Simpson (LFCC)

Bromus inermis Leyss. (smooth brome) *

- Shenandoah Co. Park, beside parking lot at forest border.; 6/15/2011; 38.93673,-78.45174;

Domangue 351

- Fort Valley, in open, weedy lot. Clay soil.; 5/25/2011; 38.88446,-78.37760; Domangue 274

- Hockman Homestead ("Chequer's") 1 mile North of Edinburg on U.S. Route 11 on east side of road; Unmowed lawn.; 6/13/1977; G. Evans, J. Evans (LFCC)

Bromus japonicus Thunb. ex Murr. (Japanese brome) *

- $\quad$ Aprox 6 miles West of Columbia Furnace on Laurel Run at the intersection of Rt. 691 and Rt 717. .2 miles West on Rt. 691; Seepage area by the edge of stream.; 6/5/1977; R.C. Simpson, K.D. Ramsey (LFCC)

- Hockman Homestead ("Chequer's") 1 mile north of Edinburg on U.S. Route 11 on east side of road; Unmowed lawn.; 6/13/1984; G. Evans, J. Evans (LFCC)

$\diamond$ Bromus latiglumis (Shear) Hitchc. (earlyleaf brome)

$\diamond$ Bromus pubescens Sprengel (hairy woodland brome)

Bromus racemosus $\mathrm{L}$. (bald brome) *

- In weedy lot at rt. 11 intersection with rt. 620.; 5/30/2011; 38.69481,-78.64817; Domangue 279

$\diamond$ Bromus secalinus L. (rye brome) *

$\diamond$ Bromus sterilis L. (poverty brome) *

Bromus tectorum L. (cheatgrass) *

- $\quad$ Strasburg, Junction of Rt 55 and Rt 623; Dry grassy field; Elev. 700 ft.; 5/23/1978; R.F. Whiting (LFCC)

- $\quad$ Fort Valley, in open, weedy lot. Clay soil.; 5/25/2011; 38.88446,-78.37760; Domangue 273

$\diamond$ Calamagrostis coarctata (Torr.) Eaton (arctic reedgrass) 
Calamagrostis porteri Gray (Porter's reedgrass)

- Rt \#675 - Wolf Gap, 1/2 mile E. of W. Va. And Va. State line; Campside next to trail - up trail to top of Big Shloss Mt.; G. Evans (LFCC)

Chasmanthium latifolium (Michx.) Yates (Indian woodoats)

- $\quad$ Fort Valley, in riparian zone near Passage Creek.; 7/29/2011; 38.88417,-78.37560; Domangue 494

$\diamond$ Chasmanthium laxum (L.) Yates (slender woodoats)

$\diamond$ Chloris verticillata Nutt. (tumble windmill grass) *

$\diamond$ Cinna arundinacea $\mathrm{L}$. (sweet woodreed)

Cynodon dactylon (L.) Pers. (Bermudagrass) *

- $\quad$ Rt. 685, 1.3 m N from Rt. 675. (NW Edinburg); Elev. 850 ft.; 6/19/1980; G. Evans, K. Ramsey, R.C. Simpson (LFCC)

- Hockman Homestead (Chequers) US Rt. 11 on east side of road; garden.; 7/9/1977; G. Evans (LFCC)

Dactylis glomerata L. (orchardgrass) *

- Devil's Backbone State Forest beside stream.; 6/8/2011; 39.04911,-78.43460; Domangue 322

- Approx 2 mi. N.W. of the Junction of \#675 and \#678 on \#675 on the Massanutten Mtn.; water trough springs.; 5/4/1977; R.C. Simpson (LFCC)

- Valley View Orchard west of Strasburg on Route 622; Open ground in apple orchard; Elev. 800'.; 8/22/1978; B. Rinker (LFCC)

+ Danthonia compressa Austin (flattened oatgrass)

- Rt. 675 - Wolfs Gap - 1/2 mi east of W. Va. State Line. Campsite next to top of Big Schloss Mt.; 7/3/1977; G. Evans (LFCC)

- $\quad$ Rt. 675, Wolfs Gap, 1/2 mi east of W. Va. And Va. State Line; campside next to trail.; 7/3/1977; G. Evans (LFCC)

Danthonia spicata (L.) Beauv. ex Roem. \& Schult. (poverty oatgrass)

- $\quad$ Little Fort Recreation trail.; 6/13/1980; R.C. Simpson (LFCC)

- Massanutten Mt., Little Fort Recreation Area, along Powell's Mt. Trail (to Edinburg Gap) about 1 mi. south of the Little Fort Camping Area; Elev. 1390 ft.; 6/26/1977; R.C. Simpson, G. Evans, D. Steed (LFCC)

Deschampsia flexuosa (L.) Trin. (wavy hairgrass)

- Woodstock, Riverview Park. Edge of lawn in lightly wooded area.; 5/18/2011; 38.87339,78.49358; Domangue 217

- $\quad$ Rt \#675 - Wolf Gap - 1/2 mile E. of W.VA and Va state line. Campside next to trail - up trail to top of Big Sholoss.; G. Evans (LFCC)

- $\quad$ Aoorx 5 mi E of Edinburg the S. of \#678 on a shale barren South of road; Elev. $2300 \mathrm{ft}$; 7 7/7/1978; R.C. Simpson (LFCC)

Dichanthelium acuminatum (Sw.) Gould \& C.A. Clark (tapered rosette grass)

- Rt. 685; 1.3 m. N from Rt 675; (NW Edinbug); Elev. 850 ft.; 6/19/1977; G. Evans, K. Ramsey, R.C. Simpson (LFCC) 
Dichanthelium boscii (Poir.) Gould \& C.A. Clark (Bosc's panicgrass)

- Woodstock, Riverview Park. Found on grassy trail in shade.; 5/30/2011; 38.87308,-78.48867; Domangue 286

$\diamond$ Dichanthelium clandestinum (L.) Gould (deertongue)

Dichanthelium commutatum (Schult.) Gould (variable panicgrass)

- $\quad$ FR 374 roadside. Silty soil.; 5/25/2011; 38.72938,-78.56740; Domangue 246

- $\quad$ Fort Valley; Powell's Fort Camp bog where the electric line cuts across the wetland.; 5/31/1977; R.C. Simpson (LFCC)

- Powell's Foot Seepage area; Mud Gap.; 6/24/1978; R.C. Simpson (LFCC)

Dichanthelium depauperatum (Muhl.) Gould (starved panicgrass)

- $\quad$ S. of Wolf Gap at Prescribed burn; Elev. 3000.; 6/15/1980; R.C. Simpson (LFCC)

- Devils Hole Mountain, Rt 691; roadside of Power site; Elev. 3,005 feet.; 7/7/1980; S. Gwyn, R.C. Simpson (LFCC)

Dichanthelium dichotomum (L.) Gould (cypress panicgrass)

- Powell's Fort anganig atcin (in Massanutten Moutain); Elev. 1500 ft.; 9/12/1978; R.C. Simpson (LFCC)

- Massanutten Mountain, Little Fort Rec. Area, along Powells mountain trail (to Edinburg Gap) approx. 1 mi. south Little Fort camping area; Along moist edge of jeep trail.; 6/26/1977; R.C. Simpson, G. Evans, D. Steed (LFCC)

$\diamond$ Dichanthelium latifolium (L.) Harvill (broadleaf rosette grass)

$\diamond$ Dichanthelium laxiflorum (Lam.) Gould (openflower rosette grass)

$\diamond$ Dichanthelium linearifolium (Scribn.) Gould (slimleaf panicgrass)

$\diamond$ Dichanthelium microcarpon (Muhl. ex Elliott) Mohlenbr. (cypress panicgrass)

$\diamond$ Dichanthelium oligosanthes (Schult.) Gould var. scribnerianum (Nash) Gould (Scribner's rosette grass)

$\diamond$ Dichanthelium polyanthes (Schult.) Mohlenbr. (roundseed panicgrass)

Dichanthelium sphaerocarpon (Ell.) Gould (roundseed panicgrass)

- 6 mi west of Columbia Furnace on Laurel Run at intersection of RT \#691 and RT \#717; Along edge of seepage pond.; 6/5/1977; R. Simpson, (LFCC)

Dichanthelium sphaerocarpon (Ell.) Gould (roundseed panicgrass)

- Massanutten Mountain, Little Fort Recreation area, along Powells mountain trail (to Edinburg Gap) approx $1 \mathrm{mi}$ south little fort camping area; Along moist edge of jeep trail.; 6/26/1977; R.C. Simpson, G. Evans, D. Steed (LFCC)

$\diamond$ Dichanthelium spretum (Schult.) Freckmann (Eaton's rosette grass)

$\diamond$ Digitaria filiformis (L.) Koeler var. filiformis (slender crabgrass) 
Digitaria ischaemum (Schreb.) Muhl. (smooth crabgrass) *

- Off rt. 620, in overgrown area beside lawn.; 8/2/2011; 38.69401,-78.64675; Domangue 503

- Growing in weedy lot at the intersection of rt. 11 and rt. 620.; 7/12/2011; 38.69518,-78.64851; Domangue 417

$\diamond$ Digitaria sanguinalis (L.) Scop. (hairy crabgrass)

Echinochloa crus-galli (L.) Beauv. (barnyardgrass) *

- $\quad$ Rt. 730 roadside at forest border.; 7/29/2011; 38.71358,-78.58472; Domangue 481

- $\quad$ Fishers Hill on Rt \#601 to 801 at Fishers Hill Dam; Dam's edge in sandy soil.; 9/17/1975; K.D. Ramsey, (LFCC)

- $\quad$ Edinburg; field; Elev. 800 ft.; 8/25/1978; B. Baker (LFCC)

$\diamond$ Echinochloa muricata (Beauv.) Fern. var. muricata (rough barnyardgrass)

Eleusine indica (L.) Gaertn. (Indian goosegrass) *

- Public boat landing at N. Fork Shenandoah River, a few meters from Narrow Passage Creek.; 8/2/2011; 38.84594,-78.52970; Domangue 513

- $1 \mathrm{mi} \mathrm{N}$ of Edinburg on US 11; Hockman Homestead; in yard near gravel driveway; Elev. $1500 \mathrm{ft}$; 8/19/1978; N. Kappel (LFCC)

Elymus hystrix L. (eastern bottlebrush grass)

- Tomahawk Pond Campground, on the edge of gravel trail near parking lot.; 7/13/2011; 38.75916, -78.84070; Domangue 444

Elymus repens (L.) Gould (quackgrass) *

- Hockman Homestead ("Chequer's") 1 mile North of Edinburg on U.S. Route 11 on East side of road; Unmowed lawn.; 6/13/1977; G. Evans, J. Evans (LFCC)

Elymus riparius Wieg. (riverbank wildrye)

- Columbia Furnace on rt. 675 roadside in weedy area a few meters from Stony Creek.; 6/8/2011; 38.87195,-78.62949; Domangue 310

Elymus villosus Muhl. ex Willd. (hairy wildrye)

- Rt. 720 roadside near Meems Bottom Covered Bridge.; 7/1/2011; 38.72057,-78.65418; Domangue 406

Elymus virginicus L. (Virginia wildrye)

- Hockman Homestead, ("Chequers") 1 mile north of Edinburg on U.S. Route 11 on east side of road; Wooded river bank grazed by cattle.; 6/13/1980; G. Evans, J. Evans (LFCC)

$\diamond$ Eragrostis capillaris (L.) Nees (lace grass)

Eragrostis cilianensis (All.) Vign. ex Janchen (stinkgrass) *

- Public boat landing at N. Fork Shenandoah River, a few meters from Narrow Passage Creek.; 8/2/2011; 38.84594,-78.52970; Domangue 507

- $1 \mathrm{mi} \mathrm{N}$ of Edinburg on US 11; Hockman Homestead; in yard near gravel driveway; Elev. 1500 ft.; 8/19/1978; N. Kappel (LFCC) 
$\diamond$ Eragrostis curvula (Schrad.) Nees (weeping lovegrass) *

$\diamond$ Eragrostis frankii C.A. Mey. ex Steud. (sandbar lovegrass)

$\diamond$ Eragrostis hypnoides (Lam.) B.S.P. (teal lovegrass)

+ Eragrostis pectinacea (Michx.) Nees (tufted lovegrass)

- Public boat landing at N. Fork Shenandoah River, a few meters from Narrow Passage Creek.; 8/2/2011; 38.84594,-78.52970; Domangue 508

$\diamond$ Eragrostis pilosa (L.) Beauv. (Indian lovegrass) *

+ Eragrostis spectabilis (Pursh) Steud. (purple lovegrass)

- Rt. 620 roadside, across from Cedar Grove Church.; 7/12/2011; 38.69439,-78.64739; Domangue 419

Festuca arundinacea Schreb. (tall fescue) *

- $\quad$ Approx. 2 miles north of Woodstock off US RT. 11 at Pugh's Run; over limstone at stream edge.; 6/5/1977; R.C. Simpson, K.D. Ramsey (LFCC)

Festuca rubra L. (red fescue)

- Massanutten Mountain .5 mi. East of the Little Fort recreation area, on Rt \#758; moist area along roadside.; 6/26/1977; R.C. Simpson, D. Steed (LFCC)

- Massanutten Mt., Little Fort Recreation Area, along Powell's Mountain Trail (to Edinburg Camp) about 1 mi. south of the Little Fort Camping Area.; 6/26/1977; R.C. Simpson, G. Evans, D. Steed (LFCC)

Festuca subverticillata (Pers.) Alexeev (nodding fescue)

- $\quad$ Shenandoah Co. Park. In lightly forested area.; 5/30/2011; 38.93653,-78.45357; Domangue 299

- $\quad$ Fort Valley, 1/4 mile E. of Glass house.; 6/22/1980; R.C. Simpson (LFCC)

Glyceria melicaria (Michx.) F.T. Hubbard (melic mannagrass)

- $\quad$ West of Edinburg GWNF; Little Sluice Trail.; 6/27/1980; R.C. Simpson (LFCC)

$\diamond$ Glyceria septentrionalis Hitchc. (floating mannagrass)

Glyceria striata (Lam.) Hitchc. (fowl mannagrass)

- Devil's Backbone State Forest. Growing in rocky, dried up stream bed.; 6/8/2011; 39.04911, -78.43460; Domangue 324

- $\quad$ Laurel Run Road, 1/2 mile west of Rt 691 on north side of road; Roadside; Elev. 1,300 feet.; 7/7/1980; R.C. Simpson, S. Gwyn (LFCC)

- $\quad$ Approx. 2 miles north of Woodstock off Rt. 11 at Pugh's Run; over lime stone.; 6/5/1977; R.C. Simpson, K.D. Ramsey (LFCC)

Holcus lanatus L. (common velvetgrass) *

- Massanutten Mt.; Little Fort Recreation Area, along Powell's Mt. Trail (to Edinburg Gap) about 1 mi. south of the Little Fort Camping Area; Elev. 1390 ft.; 6/26/1977; R.C. Simpson, G. Evans, D. Steed (LFCC)

- $\quad$ On Rt \#678 app 3 mi North of Kings Crossing.; 5/31/1977; R.C. Simpson (LFCC) 
$\diamond$ Hordeum pusillum Nutt. (little barley)

Leersia oryzoides (L.) Sw. (rice cutgrass)

- Valley View Orchard west of Strasburg on Route 622.; Open ground in apple orchard near pond; Elev. 800'.; 8/22/1978; B. Rinker (LFCC)

- Petersmill Bog, Fort Valley; Bog.; 8/16/1975; R.C. Simpson (LFCC)

Leersia virginica Willd. (whitegrass)

- Tomahawk Pond Campground on the edge of pond.; 9/8/2011; 38.75887,-78.84084; Domangue 600

- Public boat landing, beside gravel lot near Narrow Passage Creek and N. Fork Shenandoah River.; 9/1/2011; 38.84593,-78.52971; Domangue 594

Lolium perenne $\mathrm{L}$. (perennial ryegrass) *

- $\quad$ G. W. National Forest Little Fort Peters Mill Run.; 9/11/1980; R.C. Simpson, M. Willeford (LFCC)

- $\quad$ Little Fort Recreation trail.; 6/13/1980; R.C. Simpson (LFCC)

$\diamond$ Melica mutica Walt. (twoflower melicgrass)

$\diamond$ Melica nitens (Scribn.) Nutt. ex Piper (threeflower melicgrass)

Microstegium vimineum (Trin.) A. Camus (Nepalese browntop) *

- $\quad$ Rt. 610 roadside at forest border near Tomahawk Pond Campground.; 9/22/2011; 38.75840, -78.84190; Domangue 639

Muhlenbergia frondosa (Poir.) Fern. (wirestem muhly)

- $\quad$ Strasburg Park, at boat landing beside N. Fork Shenandoah River.; 9/15/2011; 38.97337, -78.35134; Domangue 632

Muhlenbergia schreberi J.F. Gmel. (nimblewill)

- $\quad$ Growing in grassy field off rt. 711.; 10/27/2011;38.79589,-78.66165; Domangue 659

- Hockman Homestead, 1 mi. North of Edinburg, U.S. Rt. 11, 15 feet west of dwelling; lawn.; 9/30/1975; G. Beaty (LFCC)

$\diamond$ Muhlenbergia sobolifera (Muhl. ex Willd.) Trin. (rock muhly)

$\diamond$ Muhlenbergia sylvatica (Torr.) Torr. ex Gray (woodland muhly)

$\diamond$ Muhlenbergia tenuiflora (Willd.) B.S.P. (slimflower muhly)

Panicum anceps Michx. (beaked panicgrass)

- Devil's Backbone State Forest, beside dirt road.; 7/20/2011; 39.04836,-78.43542; Domangue, C.K. McMullen 471

Panicum capillare L. (witchgrass)

- Rt. 610 roadside at forest border near Tomahawk Pond Campground.; 9/22/2011; 38.75840, -78.84190; Domangue 641 
Panicum dichotomiflorum Michx. (fall panicgrass)

- $\quad$ Strasburg Park, at boat landing beside N. Fork Shenandoah River.; 9/15/2011; 38.97337,78.35134; Domangue 634

- Valley View Orchard west of Strasburg on Route 622; Open ground in apple orchard; Elev. 800'.; 8/22/1978; B. Rinker (LFCC)

$\diamond$ Panicum philadelphicum Bernh. ex Trin. (Philadelphia panicgrass)

$\diamond$ Panicum rigidulum Nees (redtop panicgrass)

$\diamond$ Paspalum boscianum Flueggé (bull crowngrass)

Paspalum laeve Michx. (field paspalum)

- Rt. 620 roadside, across from Cedar Grove Church.; 7/12/2011; 38.69439,-78.64739; Domangue 420

$\diamond$ Paspalum pubiflorum Rupr. ex Fourn. (hairyseed paspalum)

$\diamond$ Paspalum setaceum Michx. (thin paspalum)

$\diamond$ Phalaris arundinacea $\mathrm{L}$. (reed canarygrass)

Phleum pratense L. (timothy) *

- $\quad$ Intersection of rt. 703 and 709. Roadside.; 6/3/2011; 38.81532,-78.66312; Domangue, M. Lantz 301

- Valley View orchard west of Strasburg on Rt. 622; Open ground in apple orchard; Elev. 800'.; 8/22/1978; B. Rinker (LFCC)

$\diamond$ Piptochaetium avenaceum (L.) Parodi (blackseed speargrass)

Poа аппиа L. (annual bluegrass) *

- $\quad$ Approx. 2 mi. N.W. of the junction of \#675 and \#678; Water trough, woodland spring.; 5/4/1977; R.C. Simpson (LFCC)

- $\quad$ Rt. 685; 1.3 mi. N from Rt. 675 N W of Edinburg; Elev. 850 ft.; 6/19/1977; G. Evans, K. Ramsey, R.C. Simpson (LFCC)

$\diamond$ Poa autumnalis Muhl. ex Ell. (autumn bluegrass)

Poa compressa L. (Canada bluegrass) *

- $\quad$ Approx. 2 miles north of Woodstock at Pugh's Run off US Rt. 11; over limestone at stream edge.; 6/5/1997; R.C. Simpson, K.D. Ramsey (LFCC)

- $\quad$ Fort Valley, 5.7 miles from Rt. 55 on 678.; 4/25/1980; R.C. Simpson (LFCC)

Poa cuspidata Nutt. (early bluegrass)

- $\quad$ Fort Valley.; 3/21/1975; R.C. Simpson (LFCC) 
Poa pratensis L. (Kentucky bluegrass)

- $\quad$ Fort Valley near Passage Creek. Growing among other grasses.; 5/4/2011; 38.88414,-78.37567; Domangue 136

- $\quad$ Power line 300 yds. E of Rt 717; 4 mi. NE of Basye; moist.; 5/18/1984; S. Arey 21 (JMUH)

Poa trivialis L. (rough bluegrass) *

- Hockman Homestead ("Chequer's") 1 mile North of Edinburg on U.S. Route 11 on East side of road; Unmowed lawn.; 6/13/1980; G. Evans, J. Evans (LFCC)

$\diamond$ Schizachyrium scoparium (Michx.) Nash var. scoparium (little bluestem)

$\diamond$ Secale cereale $\mathrm{L}$. (cereal rye) *

Setaria faberi Herrm. (Japanese bristlegrass) *

- Valley View Orchard west of Strasburg on Route 622.; Open ground in apple orchard; Elev. 800'.; 8/22/1978; B. Rinker (LFCC)

Setaria parviflora (Poir.) Kerguélen (marsh bristlegrass)

- $\quad$ Rt. 620 roadside, across from Cedar Grove Church.; 7/12/2011; 38.69439,-78.64739; Domangue 423

- Camp Roosevelt, growing in weedy gravel lot.; 6/24/2011; 38.72800,-78.51525; Domangue, J. Courtwright 366

- Hockman Homestead 1 mile north of Edinburg U.S. Rt 11. $25 \mathrm{ft}$ west of dwelling; lawn.; 9/30/1975; G. Beaty (LFCC)

Setaria pumila (Poir.) Roem. \& Schult. (yellow foxtail) *

- Rt. 610 roadside at forest border near Tomahawk Pond Campground.; 9/8/2011; 38.75932, -78.84151; Domangue 602

- Public boat landing at N. Fork Shenandoah River, a few meters from Narrow Passage Creek.; 8/16/2011; 38.84590,-78.52963; Domangue 555

- In weedy lot at rt. 11 intersection with rt. 620.; 5/30/2011; 38.69481,-78.64817; Domangue 280

- $\quad$ 1/4 mi W. of Rt. 628 at Cedar Cr.; open field; Altitude 980 ft.; 9/29/1978; E. Fowlkes (LFCC)

- Edinburg; open field; Elev. 800 ft.; 8/25/1978; B. Baker (LFCC)

Setaria viridis (L.) Beauv. (green bristlegrass) *

- 1 mile north of Edinburg U.S. Rt 1125 feet North of dwelling; lawn.; 10/2/1975; G. Beaty (LFCC)

- $1 \mathrm{mi}$ N of Edinburg on US 11; Hockman Homestead; in yard next to gravel driveway; Elev. 1500 ft.; 8/19/1978; N. Kappel (LFCC)

Sorghastrum nutans (L.) Nash (Indiangrass)

- $\quad$ Rt. 610 roadside at forest border near Tomahawk Pond Campground.; 9/22/2011; 38.75840, -78.84190; Domangue 640

- $\quad$ Fort Valley.; 3/21/1974; R.C. Simpson (LFCC)

Sorghum halepense (L.) Pers. (Johnsongrass) *

- Growing in weedy lot at the intersection of rt. 11 and rt. 620.; 7/12/2011; 38.69518,-78.64851; Domangue 416

- $\quad$ Strasburg 1mi. S on Rt. 11 on E roadside; grassy roadside.; 9/17/1975; K.D. Ramsey (LFCC) 
$\diamond$ Sphenopholis nitida (Biehler) Scribn. (shiny wedgescale)

$\diamond$ Sphenopholis obtusata (Michx.) Scribn. (prairie wedgescale)

$\diamond$ Sphenopholis pensylvanica (L.) Hitchc. (swamp wedgescale)

$\diamond$ Sporobolus compositus (Poir.) Merr. var. compositus (composite dropseed)

Sporobolus vaginiflorus (Torr. ex Gray) Wood (poverty dropseed)

- $\quad$ Strasburg Park, beside gravel parking lot.; 9/15/2011; 38.97377,-78.35139; Domangue, 631

Tridens flavus (L.) Hitchc. (purpletop tridens)

- $\quad$ Strasburg Park, on the edge of grassy field.; 9/15/2011; 38.97580,-78.35493; Domangue 627

- $\quad$ Elizabeth Furnace Recreation Area on the edge of parking lot.; 8/23/2011; 38.92783,-78.32947; Domangue 584

- $\quad$ Open field; Elev. 800 ft.; 8/25/1978; B. Baker (LFCC)

- 1 mile north of Edinburg U.S. Rt 1110 feet North of dwelling; lawn.; 10/2/1975; G. Beaty (LFCC)

$\diamond$ Tripsacum dactyloides $(\mathrm{L}.) \mathrm{L}$. (eastern gamagrass)

$\diamond$ Vulpia octoflora (Walt.) Rydb. (sixweeks fescue)

Pontederiaceae

Heteranthera dubia (Jacq.) MacMill. (grassleaf mudplantain)

- $\quad$ N.E. end Edinburg; Jct. Stoney Creek + Shen. Rt. 675; Elev. 850 ft.; 6/19/1977; G. Evans, K. Ramsey, R.C. Simpson (LFCC)

- In Shenandoah River near Maurertown, VA.; 8/17/1983; D. Russell Jr. (LFCC)

Potamogetonaceae

Potamogeton amplifolius Tuck. (largeleaf pondweed)

- Aprox. 6 miles West of Columbia Furnace on Laruel Run at the intersection of Rt 691 and Rt. 717 .2 miles west on Rt. 691(in seepage ponds); seepage and damed ponds 1 feet to several feet.; 6/5/1977; R.C. Simpson, K.D. Ramse (LFCC)

Potamogeton crispus L. (curly pondweed) *

- Fishers Hill Dam, Fishers Hill; 2 mi. south of strasburg. Growing in large quanitities in the Dam's water.; 4/30/1975; K. Ramsey (LFCC)

- $\quad$ N.E. end of Edinburg, JCT. Stoney Creek . A Shen. River. Rt. 675; Elev. 850 ft.; 6/19/1977;

G. Evans, K. Ramsey, R.C. Simpson (LFCC)

Potamogeton diversifolius Raf. (waterthread pondweed)

- Massanutten Moutain, Mine Run Estates; pond.; 8/23/1978; R.C. Simpson (LFCC)

$\diamond$ Potamogeton illinoensis Morong (Illinois pondweed)

$\diamond$ Potamogeton nodosus L. (longleaf pondweed) 
$\diamond$ Stuckenia pectinata (L.) Borner (sago pondweed)

Smilacaceae

Smilax glauca Walt. (cat greenbrier)

- $\quad$ Fort Valley Fish Hatchery; Elev. 1400 ft.; 10/27/1974; R.C. Simpson (LFCC)

- Massanutten Mt.; Little Fort Recreation Area, along Powell's Mt. Trail (to Edinburg Gap) about 1 mi. south of the Little Fort Camping Area; Elev. 1390 ft.; 6/26/1977; R.C. Simpson, G. Evans, D. Steed (LFCC)

Smilax herbacea $\mathrm{L}$. (smooth carrionflower)

- Moist meadows; 678 Fort Valley.; 5/21/1986; (JMUH)

- Strasburg, Intersection of Rt 55 Shenandoah Press parking lot across the street; Roadside fauna; Elev. 850 ft.; 10/13/1979; R. Bowden (LFCC)

Smilax rotundifolia $\mathrm{L}$. (roundleaf greenbrier)

- $\quad$ Red Robin Ln. roadside, in weedy area.; 7/20/2011; 38.94599,-78.52652; Domangue, C.K. McMullen 475

- Tomahawk Pond Campground. Viny plant growing in wooded area over Dogwood tree; 5/10/2011; 38.75795,-78.84186; Domangue, B. Burkholder 160

- Ap. 2 miles east of Edinburg on \#675 where power line crosses road; roadside.; 5/4/1977; R. Simpson (LFCC)

- $\quad$ Paddy's Run 4 mi W of Star Tannery off of Rt 55; moist mountain stream area; Elev. $900 \mathrm{ft}$; 9/9/1978; J. Macdonell (LFCC)

$\diamond$ Smilax tamnoides L. (bristly greenbrier)

Sparganiaceae

+ Sparganium americanum Nutt. (American bur-reed)

- $\quad$ Passage Creek; Stream Bank; Altitude 800.; 8/2/1965; L. Artz (JMUH)

\section{Typhaceae}

Typha latifolia $\mathrm{L}$. (broadleaf cattail)

- Tomahawk Pond Campground, growing on the edge of pond. $1.5 \mathrm{~m}$ tall.; 7/13/2011; 38.75898, -78.84057; Domangue 442

- 2 mi South of Strasburg growing in good size stand on west side of Dam. Fishers Hill Dam, Fishers Hill VA.; 4/30/1975; K. Ramsey (LFCC)

\section{Zannichelliaceae}

$\diamond$ Zannichellia palustris L. (horned pondweed) 


\section{Dicots}

\section{Acanthaceae}

Justicia americana (L.) Vahl (American water-willow)

- $\quad$ Growing on the edge of the N. Fork Shenandoah River off Black Bear Rd.; 7/20/2011; 38.90966, -78.42236; Domangue, C.K. McMullen 458

- $\quad$ Elizabeth Furnace Recreation Area. Growing in Passage Creek.; 6/24/2011; 38.92935,-78.32882; Domangue, J. Courtwright 380

- $\quad$ N.E. end of Edinburg; JCT. Stoney Creek and Shen. River.; Elev. 850 ft.; 6/19/1977; G. Evans, K. Ramsey, R.C. Simpson (LFCC)

Ruellia caroliniensis (Gmelin) Steud. (Carolina wild petunia)

- Approx. 2 miles north of Woodstock off US Rt 11 at Pugh's Run; over limestone at edge of stream.; 6/5/1977; R.C. Simpson, K.D. Ramsey (LFCC)

- $\quad$ Side of road, rocky; 1 mil S. of Strasburg on U.S. Rt. \#11 right hand side of road.; Flowers: lavender.; 6/1/1975; K. Ramsey (LFCC)

$\diamond$ Ruellia humilis Nutt. (fringeleaf wild petunia)

$\diamond$ Ruellia purshiana Fernald (Pursh's wild petunia)

Ruellia strepens L. (limestone wild petunia)

- $\quad$ Cedar Grove Church, on the edge of parking lot and grassy field.; 5/30/2012; 38.69425,-78.64771; Domangue 702

- $\quad$ Edge of public boat landing parking lot, near N. Fork Shenandoah River.; 6/15/2011; 38.84590, -78.52994; Domangue 343

Aceraceae

Acer negundo L. (boxelder)

- $\quad$ Growing on the N. Fork Shenandoah River floodplain off Black Bear Rd.; 7/20/2011; 38.90966, -78.42236; Domangue, C.K. McMullen 452

- $\quad$ Fort Valley near Passage Creek. Large tree.; 5/4/2011; 38.88414,-78.37567; Domangue 135

- $\quad$ Little Fort Valley, East of Powell's Fork Camp; at Mud Hole Bog.; 6/6/1905; R. Berg, S. Chappell 108 (JMUH)

- 1 mi. so. Strasburg, 3/4 mi S. East of intersection of Rt. 11 on 601.; Elev. 575 ft.; 8/7/1979; P. Johns (LFCC)

- $\quad$ Edinburg; Elev. 740 ft.; 4/12/1978; K. Halstead (LFCC)

Acer nigrum Michx. f. (black maple)

- 2 miles South of Mt. Olive on West side of Rt. 623. In flatwoods; Oak-Hickory forest; Elev. 1000 ft.; 10/15/1978; C. Whittington (LFCC) 
Acer pensylvanicum $\mathrm{L}$. (striped maple)

- Tomahawk Pond Campground. Small tree in lightly forested area.; 5/10/2011; 38.75906, -78.84145; Domangue, B. Burkholder 165

- 675 west of Columbia Furnace on Forestry Access Road (Little Stoney) 6 miles east; East slope; Elev. 1800 ft.; 10/10/1984; A.D. Wolverton (LFCC)

- $\quad$ Go Rt 42-S for 5.7 miles until it intersects with Rt. 675-W. Go West on Rt 675 until you come to Wolf Gap recreation area. Specimens collected along Big Blue hiking trail.; Oak-Hickory forest. Dry mesic conditions.; Elev. 2600 ft.; 10/8/1978; L. Leta (LFCC)

Acer platanoides L. (Norway maple) *

- Woodstock, Riverview Park on edge of Effinger Trail. Large tree.; 5/18/2011; 38.87228, -78.49006; Domangue 224

- LB's - Intersection of Rt 11 and Rt. $627 \mathrm{~W}$ of Middletown; $3.5 \mathrm{~m}$ go left .2 mi. to house; In yard; Elev. 760.; 9/29/1979; P. Johns (LFCC)

- $\quad$ Rt. \#11 at junction with Cedar Creek; Growing on creek bank; Elev. 594 ft.; 5/22/1978;

K. Halstead (LFCC)

Acer rubrum L. (red maple)

- On route 600 roadside on the edge of the woods. Large tree.; 5/10/2011; 38.97044,-78.52255;

Domangue, B. Burkholder 171

- 2 miles from West Virginia Line and Wolf Gap Rec. area on left side road; mixed hard woods; Elev. 2400 ft.; 10/8/1978; A. Orndorff (LFCC)

- East of Strasburg on route 678, north end of Massanutten Mt. in Fort Valley at Elizabeth Furnace Campground.; Growing in understory of Oak hickory forest.; Elev. 800'.; 10/3/1977; O. Bate (LFCC)

Acer saccharinum $\mathrm{L}$. (silver maple)

- $\quad$ Street.; 9/1/1986; D. Fadely (LFCC)

- $\quad$ Maurertown, RT \#1 Box 197; Fence Row.; 10/3/1978; M. Bowman (LFCC)

Acer saccharum Marsh. (sugar maple)

- Tomahawk Pond Campground. Large tree at the edge of parking lot.; 5/10/2011; 38.75883, -78.84087; Domangue, B. Burkholder 167

- In the yard at 5875 Gospel St. Mt. Jackson.; 3/18/1990; J.E. Irre 18 (JMUH)

- 2 miles south of Mt. Olive on RT. 623 on west side of road in flat woods.; oak-hickory forest; Elev. 1000 ft.; 10/15/1978; C. Whittington (LFCC)

$\diamond$ Acer spicatum Lam. (mountain maple)

Adoxaceae

Sambucus canadensis L. (American black elderberry)

- Rt. 698 roadside, in open area next to cultivated field. Shrub.; 6/15/2011; 38.81675,-78.57158; Domangue 340 
Viburnum acerifolium L. (mapleleaf viburnum)

- Woodstock, Riverview Park. Small tree, 2 m tall, on steep slope in light woods.; 5/18/2011; 38.87339,-78.49358; Domangue 216

- $\quad$ Rte. 675 Bowman's Crossing, 3.5 miles from Edinburg.; shaded area near shale barren.; 7/2/1979; VA Flora Class 18 (JMUH)

- $\quad$ Dry woods, rock; Rt. 678 Fort Valley.; (JMUH)

- $\quad$ Intersection Rt 11 So Rt $627 \mathrm{~W}$ of Middletown; 3.5 mi go left .2 mi to house; Elev. 760.; 9/29/1979; P. Johns (LFCC)

- $\quad$ At Little Fort on the Massanutten Mt.; 6/19/1975; R.C. Simpson (LFCC)

Viburnum dentatum L. (southern arrowwood)

- $\quad$ Mudhole Bog by Little Passage Creek in Little Fort Valley.; Growing in a boggy area along a stream.; 6/28/1972; BIO 68517 (JMUH)

- $\quad$ Fort Valley at Little Fort on Peter's Mill Run.; 6/20/1975; R.C. Simpson (LFCC)

- $\quad$ Toms Brook; 9/9/1980; R.C. Simpson (LFCC)

Viburnum dentatum L. var. lucidum Aiton (southern arrowwood)

- $\quad$ Little Fort Campground in forest. Small tree.; 8/23/2011; 38.86774,-78.44334; Domangue 585

$\diamond$ Viburnum nudum L. (possumhaw)

Viburnum prunifolium L. (blackhaw)

- $\quad$ In cow pasture of weeds and woody plants. $3 \mathrm{~m}$ tall shrub.; 4/11/2012; 38.79420,-78.66107; Domangue 671

- $\quad$ Fort Valley, at the edge of open pasture in weedy area near stream. Small tree.; 4/26/2011; 38.88191,-78.37437; Domangue, M. Lantz 117

- $\quad$ Ap. 2 miles east of Edinburg, where power line crosses road; Roadside.; 5/4/1977; R. Simpson (LFCC)

- $\quad$ Found on Massanutten Mountain at the Woodstock Tower. Flowers are yellow.; 5/4/1975; R.C. Simpson (LFCC)

$\diamond$ Viburnum rafinesquianum J. A. Schultes (downy arrowwood)

Amaranthaceae

$\diamond$ Amaranthus albus L. (prostrate pigweed) *

Amaranthus hybridus L. (slim amaranth)

- $\quad$ Edinburg; open field; Elev. 800 ft.; 8/25/1978; B. Baker (LFCC)

Amaranthus retroflexus L. (redroot amaranth) *

- Public boat landing at N. Fork Shenandoah River, a few meters from Narrow Passage Creek.; 8/2/2011; 38.84594,-78.52970; Domangue 510

Amaranthus spinosus L. (spiny amaranth) *

- Growing in riparian zone at Meems Bottom Covered Bridge.; 7/12/2011; 38.72069,-78.65476; Domangue 425 
Anacardiaceae

Rhus aromatica Aiton (fragrant sumac)

- On Rt. 758 at the bottom of the mountain down the path beside the Leisure Point Sign; habitat: tall deciduous; Elev. 808 ft.; 7/4/1979; W. Rush (LFCC)

- $\quad$ Turkey run, Rt. 55; roadside.; 10/16/1975; Dendrology Class (LFCC)

Rhus copallinum L. (winged sumac)

- $\quad$ Elizabeth Furnace; Shale Barrens.; 7/13/1979; R.C. Simpson (LFCC)

- 2 mi. east of Woodstock on top of the Massanutten Mountain on the path going to the Woodstock Tower.; Along well travelled path through Quercus prinus forest; Elev. 1700'.; 9/1/1977;

B. Delph, R.C. Simpson (LFCC)

Rhus glabra L. (smooth sumac)

- $\quad$ Rt. 620 roadside along weedy fencerow. Small tree.; 8/2/2011; 38.69456,-78.64767; Domangue 501

- Cedar Grove Church, beside parking lot in weedy patch.; 6/15/2011; 38.69409,-78.64780; Domangue 328

- $\quad$ FR 274 west.; 7/22/1981; D.E. Miller 12 (JMUH)

- Cedar Creek wayside 3 mi. N. of Strasburg on Rt. 11; flood plain.; 10/2/1975; R. Brown, R. Crowder (LFCC)

- $\quad 2$ mis. East of intersection Rt. 758 and Rt. 678.; along road side.; 10/14/1979; J. Evans (LFCC)

$\diamond$ Rhus hirta (L.) Sudw. (staghorn sumac)

Toxicodendron pubescens Mill. (Atlantic poison oak)

- Turn right off of Route 11 North onto Rt. 650. Proceed 2.7 miles on left side along road.; along side dirt road.; Elev. 780'.; 5/3/1978; R. Olinger (LFCC)

Toxicodendron radicans (L.) Kuntze (eastern poison ivy)

- $\quad$ Fort Valley Massanutten Mts.; Along Ft. Valley Rd 8 mi N. of Detrick.; 5/18/1989; N.L. Bodkin (JMUH)

- Passion Theatre.; 10/10/1974; (LFCC)

Annonaceae

Asimina triloba (L.) Dunal (pawpaw)

- Clearing in wooded area near small cabin. Small tree.; 5/11/2011; 38.94997,-78.52692; Domangue, C.K. McMullen 189

- Cedar Cr.; Stream Bank.; 7/3/1964; L. Artz (JMUH)

- Cedar Creek wayside 3 mi. N. of Strasburg on Rt. 11; flood plains.; 10/2/1975; R. Brown, R. Crowder (LFCC)

- Woodstock; Woodstock Dam; along Shenandoah River Dam; Elev. 1300; 10/7/1979; J. Evans (LFCC) 
Apiaceae

Anethum graveolens L. (dill) *

- $\quad$ Found in a much used garden (along the edges) along road No. 703, located 6 miles outside of Woodstock. Residents stated it has been growing there for numerous years.; 6/30/1977; T. Burke 13 (JMUH)

$\diamond$ Angelica venenosa (Greenway) Fern. (hairy angelica)

Bupleurum rotundifolium L. (hare's ear) *

- $\quad$ Strasburg, Junction of Rt 55 and Rt. 623.; Dry grassy field; Elev. 700 ft.; 5/23/1978; R.F. Whiting (LFCC)

$\diamond$ Chaerophyllum procumbens (L.) Crantz var. procumbens (spreading chervil)

Cicuta maculata L. (spotted water hemlock)

- $\quad$ Tomahawk Pond Campground, growing on the edge of pond.; 7/13/2011; 38.75838,-78.84052; Domangue 439

- $\quad$ Fort Valley near Passage Creek in weedy area.; 6/24/2011; 38.88412,-78.37559; Domangue, J. Courtwright 390

- $\quad$ Fort Valley; Stream bank; Altitude 800.; 7/21/1965; L. Artz (JMUH)

- $\quad$ Kings Crossing, massanutten.; 6/26/1975; R.C. Simpson (LFCC)

- $\quad$ Little Fort Recreation trail.; 6/13/1980; R.C. Simpson (LFCC)

Conium maculatum L. (poison hemlock) *

- $\quad$ In weedy lot at rt. 11 intersection with rt. 620. $1.5 \mathrm{~m}$ tall. Pungent odor when crushed.; 5/30/2011; 38.69500,-78.64896; Domangue 276

- Watery Street x Main Street in Edinburg Va. 1/4 mile South of US Rt. 11 and Co. Rt. 675.; 6/21/1977; G. Evans (LFCC)

- Hockman Homestead, ("Chequers") 1 mile north of Edinburg on US. Route 11 on east side of road; Wooded river bank, grazed by cattle.; 6/13/1980; G. Evans (LFCC)

Cryptotaenia canadensis (L.) DC. (Canadian honewort)

- $\quad$ Strasburg Park, a couple meters from N. Fork Shenandoah River.; 8/2/2011; 38.97442,-78.35439; Domangue 518

- $\quad$ Rt. 672 roadside near N. Fork Shenandoah River.; 7/12/2011; 38.84435,-78.53171; Domangue 428

- Hockman Homestead ("Chequers") 1 mile north of Edinburg on U.S. Route 11 on east side of road; Wooded river bank, grazed by cattle.; 6/13/1980; G. Evans (LFCC)

- $\quad$ NE End Edinburg JCT. Stoney Creek and Shen. River, Rt. 675.; 6/19/1977; G. Evans, K. Ramsey, R.C. Simpson (LFCC) 
Daucus carota L. (Queen Anne's lace) *

- Fort Valley near Passage Creek in weedy area.; 6/24/2011; 38.88412,-78.37611; Domangue, J. Courtwright 391

- Camp Roosevelt, growing in weedy gravel lot.; 6/24/2011; 38.72800,-78.51525; Domangue, J. Courtwright 365

- Hockman Homestead 1 mile north of Edinburg. U.S. Rt 11. 150 yds south of dwelling; open field.; 10/5/1975; G. Beaty (LFCC)

- Rt. 608 at Columbia Furnace along roadside which leads to Wolf Gap.; 10/19/1978; M.L. Snarr (LFCC)

Heracleum maximum Bartr. (common cowparsnip)

- $\quad$ Elizabeth Furnace Recreation Area near Passage Creek. $1.5 \mathrm{~m}$ tall.; 5/25/2011; 38.92931, -78.32938; Domangue 260

Hydrocotyle americana $\mathrm{L}$. (American marshpennywort)

- Vance's Cove; Bog; Altitude 1400.; 7/14/1965; L. Artz (JMUH)

Osmorhiza claytonii (Michx.) C.B. Clarke (Clayton's sweetroot)

- $\quad$ Fort Valley area.; 5/18/1984; BIO 501137 (JMUH)

- $\quad$ Fishers Hill; N. slope wooded .25 mi E of US 11.; 4/28/1976; C.R. Hupp (LFCC)

Osmorhiza longistylis (Torr.) DC. (longstyle sweetroot)

- $\quad$ Shenandoah Co. Park. Edge of grass trail in light forest. $1 \mathrm{~m}$ tall.; 5/18/2011; 38.93671, -78.45296; Domangue 237

- $\quad$ Fort Valley; Dry woods.; 5/16/1964; L. Artz (JMUH)

- North of Tom's brook on \#651 about .5 mi; old overgrown woodland trail.; 5/4/1977; R.C. Simpson (LFCC)

- North of tom's brook on \#651 about .5 mi west of \#11 (between \#11 and \#81); old overgrown woodland trail.; 5/4/1977; R.C. Simpson (LFCC)

Oxypolis rigidior (L.) Raf. (stiff cowbane)

- $\quad$ Little Fort; Bog; Altitude 1000 ft.; 9/13/1963; L. Artz (JMUH)

Pastinaca sativa $\mathrm{L}$. (wild parsnip) *

- $\quad$ Rt. 720 roadside near Meems Bottom Covered Bridge.; 7/1/2011; 38.72057,-78.65418; Domangue 407

$\diamond$ Sanicula canadensis L. var. canadensis (Canadian blacksnakeroot)

Sanicula marilandica L. (Maryland sanicle)

- $\quad$ Same side of parking lot at Signal Knob parking lot.; deciduous forest; Elev. 600 ft.; 5/22/1978; H.E. Reed (LFCC)

+ Sanicula odorata (Raf.) Pryer \& L.R. Phillippe (clustered blacksnakeroot)

- Mine Mountain Rd., on roadside at forest border.; 5/25/2011; 38.89643,-78.40716; Domangue 266

$\diamond$ Sanicula trifoliata Bickn. (largefruit blacksnakeroot) 
Taenidia integerrima (L.) Drude (yellow pimpernel)

- Growing in the middle of dirt road in forest.; 5/11/2011; 38.94931,-78.52658; Domangue, C.K. McMullen 206

- $\quad$ Power line 300 yds. E. of Rt. 717; 4 mi. NE. of Basye; shady, edge of woods.; 5/15/1984; S. Arey 13 (JMUH)

- $\quad$ Fort Valley on \#678, 4 - 5 mi SE of \#55.; 3/17/1978; R.C. Simpson, J. Bursey (LFCC)

- Wooded Roadside (rocky); Massanutten Mtn. Road near Woodstock Tower; Flower - yellow.; 5/5/1975; R. \& H. Simpson (LFCC)

Taenidia montana (Mack.) Cronq. (mountain pimpernel)

- 3.7 mi. E on Rt. 675 past Edinburg; shale barren.; 5/18/1983; BIO 50191 (JMUH)

Thaspium barbinode (Michx.) Nutt. (hairyjoint meadowparsnip)

- $\quad$ Power line 300 yds. E of 717, 4 mi NE of Basye; moist open area.; 5/23/1984; S. Arey 34 (JMUH)

- $\quad$ Peters Mill, Little Fort.; 6/20/1975; R.C. Simpson (LFCC)

Thaspium trifoliatum (L.) Gray (purple meadowparsnip)

- Route 675 near entrance to Powell trail, Massanutten Mountain; Woods.; 5/16/1982; L. Michael Hill 2 (BDWR)

$\diamond$ Torilis arvensis (Huds.) Link ssp. arvensis (spreading hedgeparsley) *

Torilis japonica (Houtt.) DC. (erect hedgeparsley) *

- Cedar Cr.; Meadow.; 7/3/1964; L. Artz (JMUH)

Zizia aptera (Gray) Fern. (meadow zizia)

- $\quad$ Orkney Springs. Junction Routes 723 and 7200.1 mi. on Rt. 723; Roadside Moss in wooded area.; 5/23/1981; E. Lerch (LFCC)

Zizia aurea (L.) W.D.J. Koch (golden zizia)

- Approx. 2 mi. N.W. of the Junction of \#678 and \#675 on \#675; on the Massanutten Mountain; Shale barrens.; 5/4/1977; R.C. Simpson (LFCC)

Apocynaceae

Apocynum androsaemifolium L. (spreading dogbane)

- Ft. Valley, Little Ft. Recreation area.; Flowers: white / pink.; 6/19/1975; R.C. Simpson (LFCC)

- Massanutten Mountain, Little Fort Recreation area, along Powell's Mountain Trail (to Edinburg Gap) about 1 mi. South of the Little Fort Camping Area.; Along Dry Jeep Trail.; 6/26/1977; R.C. Simpson, G. Evans (LFCC)

Apocynum cannabinum L. (Indianhemp)

- $\quad$ Rt. 620 roadside, adjacent open field. $1 \mathrm{~m}$ tall; 6/15/2011; 38.69464,-78.64809; Domangue 332

- $\quad$ FR 274 west.; 7/22/1981; D.E. Miller 7 (JMUH)

- $\quad$ Edinburg; open field; Elev. 800 ft.; 8/25/1978; B. Baker (LFCC) 
Vinca minor L. (common periwinkle) *

- Wolf Gap recreation area in lightly forested location. Dense population.; 4/11/2011; 38.92385, -78.68866; Domangue 85

\section{Aquifoliaceae}

$\diamond$ Ilex montana Torr. \& Gray ex Gray (mountain holly)

Ilex opaca Ait. (American holly)

- $\quad$ Woodstock, Riverview Park, on forest trail. Medium-sized tree.; 8/16/2011; 38.87277,-78.48944;

Domangue 558

- $\quad$ Behind white house at 5875 Gospel St., Mt. Jackson, VA.; 2/20/1990; J.E. Irre 3 (JMUH)

- Fort Valley, Powell's Fort Camp, wet area on east side of road across from the camp.; not planted growing wild in woods.; 3/20/1977; R.C. Simpson, C.H. Hupp (LFCC)

Ilex verticillata (L.) Gray (common winterberry)

- Fort Valley, River.; 10/27/1974; R.C. Simpson (LFCC)

- $\quad$ Little Fort Recreation Area, off Rt \#758; Elev. 1400 ft.; 10/8/1981; J.A. Hepner (LFCC)

Araliaceae

$\diamond$ Aralia hispida Vent. (bristly sarsaparilla)

Aralia nudicaulis L. (wild sarsaparilla)

- Rt \#691, Bear Oak Prescribed Burn, near Va. - W. Va. state line; along roadbank of burned area; Elev. 3005 feet.; 5/22/1981; S. Collins (LFCC)

$\diamond$ Aralia racemosa $\mathrm{L}$. (American spikenard)

$\diamond$ Aralia spinosa L. (devil's walkingstick)

Aristolochiaceae

Aristolochia serpentaria L. (Virginia snakeroot)

- $\quad$ At Little Fort on the Massanutten.; 6/19/1975; R.C. Simpson (LFCC)

$\diamond$ Asarum canadense L. (Canadian wildginger)

\section{Asclepiadaceae}

Ampelamus laevis (Michx.) Krings (honeyvine)

- Rt. 672 roadside near public boat landing at N. Fork Shenandoah River.; 8/2/2011; 38.84556, -78.53018; Domangue 504

Asclepias amplexicaulis $\mathrm{Sm}$. (clasping milkweed)

- $\quad$ Fort Valley; Peters Mill Bog.; 6/24/1980; R.C. Simpson (LFCC) 
Asclepias amplexicaulis $\mathrm{Sm}$. (clasping milkweed)

- Massanutten Mountain, Little Fort Recreation area, along Powell's Mountain Trail (to Edinburg Gap) about 1 mi. South of the Little Fort Camping Area.; Along Dry Jeep Trail.; 6/26/1977; R.C. Simpson, G. Evans (LFCC)

Asclepias exaltata L. (poke milkweed)

- $\quad$ FR 374 roadside bank at forest border. $1 \mathrm{~m}$ tall. Milky sap.; 5/30/2012; 38.75823,-78.55400; Domangue 704

- $\quad$ Powell's Fort seepage area; Mud hole gap.; 6/24/1978; R.C. Simpson (LFCC)

Asclepias incarnata L. (swamp milkweed)

- In cow pasture next to stream. $1 \mathrm{~m}$ tall.; 6/30/2011; 38.79452,-78.65999; Domangue 402

$\diamond$ Asclepias purpurascens $\mathrm{L}$. (purple milkweed)

Asclepias quadrifolia Jacq. (fourleaf milkweed)

- $\quad$ Rt. 730 on forest border in clearing below power lines. Fragrant flowers. Sap not milky.; 5/25/2011; 38.71313,-78.58601; Domangue 245

- Fort Valley; Dry woods.; 5/18/1964; L. Artz (JMUH)

- Woodstock at Woodstock Tower; mountain path.; 6/8/1975; C. Hupp (LFCC)

- $\quad$ By Signal Knob Trail parking lot; Edge of woods; Elev. 600.; 6/1/1978; H.E. Reed (LFCC)

Asclepias syriaca $\mathrm{L}$. (common milkweed)

- $\quad$ Rt. 620 roadside, adjacent open field. 1.5 m tall; 6/15/2011; 38.69464,-78.64809; Domangue 333

- $\quad$ Kings Crossing, Massanutten.; fls. deep red.; 6/26/1975; R.C. Simpson (LFCC)

- $\quad$ Rt. 648 along roadside at Sandy Hook, Strasburg.; 10/22/1978; M.L. Snarr (LFCC)

Asclepias tuberosa L. (butterfly milkweed)

- Rt. 709 roadside next to cultivated field.; 6/30/2011; 38.80688,-78.66122; Domangue 405

- $\quad$ Fort Valley, Rt. 678; Found along roadside, dry east-facing bank.; 6/22/1977; S.E. Grimes 7 (JMUH)

- Up the bank on right hand side of Rt. 678, 7.9 mi S. of the intersection of Rt. 678 and Rt. 771; open areas, grassy fields.; 6/22/1977; B. Wiley 8 (JMUH)

- Valley View Orchard on Route 622 west of Strasburg; open roadside bordering apple orchard; Elev. 800'.; 8/25/1978; B. Rinker (LFCC)

$\diamond$ Asclepias variegata L. (redring milkweed)

Asclepias verticillata $\mathrm{L}$. (whorled milkweed)

- $\quad$ Approx $5 \mathrm{mi}$ E of Edinburg then S. of \#678 on a shale barren South of road; Elev. $2300 \mathrm{ft}$;; 7/7/1978; R.C. Simpson (LFCC)

Asclepias viridiflora Raf. (green comet milkweed)

- $\quad$ Rt. 11 roadside along fencerow.; 7/20/2011; 38.66240,-78.66306; Domangue, C.K. McMullen 447 
Matelea carolinensis (Jacq.) Woods. (maroon Carolina milkvine)

- $\quad$ Rt. \#678 Fort Valley; 200 yds north of Seven Fountain Post office (east side of road); Elev. 700 ft.; 9/9/1980; M. Krouse (LFCC)

- $\quad$ East of wheatfield on \#623.; 6/15/1980; R.C. Simpson (LFCC)

Matelea obliqua (Jacq.) Woods. (climbing milkvine)

- $\quad$ Side of road, growing near honeysuckle; Fishers Hill, 2 mi. S.W. of Strasburg, near the Old Mill.; Flowers: maroon.; 6/7/1975; K. Ramsey (LFCC)

Asteraceae

Achillea millefolium L. (common yarrow)

- $\quad$ FR 374 roadside. Silty soil.; 5/25/2011; 38.72938,-78.56740; Domangue 247

- $\quad$ FR 274 West.; 6/24/1981; D.E. Miller 52 (JMUH)

- Woodstock; Turn off Rt. 11 and go 1 1/4 miles East on Lakeview Road and take left on old dirt road and go 3/4 miles; River bottom meadow.; 7/5/1982; J. Nutter 15 (JMUH)

- $\quad$ 1/4 mi W. of Rt. 628 at Cedar Cr.; open meadow sunny field; white bloom; Altitude 950 ft.; 9/26/1975; E. Fowlkes (LFCC)

- Hockman Homestead ("Chequers") 1 mile north at Edinburg on U.S. Route 11 on east side on road; Open field grazed by cattle.; 6/17/1929; G. Evans (LFCC)

Ageratina altissima (L.) King \& H. Rob. (white snakeroot)

- $\quad$ Eliz. Furn. Rec. Area; Richwoods; Altitude 800 feet.; 8/11/1966; M. Krouse (LFCC)

- $\quad$ Rt \#691, Bear Oak Prescribed Burn, near Va. - W. Va. state line; Burned area.; 5/22/1981; S. Collins (LFCC)

Ageratina aromatica (L.) Spach (lesser snakeroot)

- 1 mile E. of Liberty Furnace at the junction of Rt. 717 and 690. Stony Creek flood plain.; 9/7/1975; R.C. Simpson (LFCC)

- $\quad$ Powell's Fort Organization Camp (in massanutten mts) on Rt 771; Elev. 1500 ft.; 9/12/1978; R.C. Simpson (LFCC)

Ambrosia artemisiifolia L. (annual ragweed)

- $\quad$ Growing in grassy field off rt. 711.; 10/27/2011; 38.79589,-78.66165; Domangue 658

- $\quad$ In weedy lot at the intersection of rt. 11 and rt. 620. Clay soil.; 9/1/2011; 38.69508,-78.64852; Domangue 587

- $\quad$ Edinburg; open field; Elev. 800 ft.; 8/25/1978; B. Baker (LFCC)

- $\quad$ Rt. 648 along roadside at Sandy Hook, Strasburg.; 10/22/1978; M.L. Snarr (LFCC)

Ambrosia trifida L. (great ragweed)

- $\quad$ Below Meems Bottom Covered Bridge at N. Fork Shenandoah River. 2 m tall.; 8/16/2011; 38.72073,-78.65482; Domangue 549

$\diamond$ Antennaria howellii Greene ssp. neodioica (Greene) Bayer (Howell's pussytoes)

Antennaria neglecta Greene (field pussytoes)

- Camp Caroline Furnace, Fort Valley, (7 miles SE of Edinburg on Route 675). 100 yards from entrance of Nature Trail, on the right.; Dry, rocky soil in open woods.; 4/23/1979; B.E. Brandt 17 (JMUH) 
Antennaria parlinii Fern. ssp. parlinii (Parlin's pussytoes)

- Camp Roosevelt at small clearing in forest.; 5/25/2011; 38.72929,-78.51756; Domangue 254

Antennaria plantaginifolia (L.) Richards. (woman's tobacco)

- Camp Roosevelt at small clearing in forest.; 5/25/2011; 38.72929,-78.51756; Domangue 253

- Devil's Backbone State Forest. Edge of dirt road. Rocky soil.; 4/18/2011; 39.04799,-78.43493; Domangue, J. Courtwright 96

- W. of King's Crossing along Rt. 675. 5 mi east of Edinburg.; rocky, dry soil.; 4/25/1983; Parker 21 (JMUH)

- Power line 300 yds E. of Rt. 717; 4 mi. NE. of Basye; dry, open areas.; 5/10/1984; S. Arey 10 (JMUH)

- Fort Valley, Camp Caroline Furnace (7 miles SE of Edinburg on Route 675). 75 yards from entrance of Nature Trail, on right side of trail.; Dry, rocky, open woods.; 4/23/1979; B.E. Brandt 19 (JMUH)

- George Washington Nat'l Forest, Elizabeth Furnace, on Rt. 678, 5 miles South of junction of Rt. 55 and Rt. 678; Field; Elev. 800 ft.; 5/22/1978; R.F. Whiting (LFCC)

- Fishers Hill Dam; Fishers Hill, 2 mi. south of Strasburg, growing on a rocky south facing slope.; 4/30/1975; K. Ramsey (LFCC)

Antennaria virginica Stebbins (shale barren pussytoes)

- $\quad$ Fort Valley Shale Barren.; 5/1/1992; (JMUH)

- 3.7 mi. east of junction of Rt. 11 and Rt. 675 in Edinburg on Rt. 675.; South facing shale barren slope.; 4/12/1982; J. Andrews 31 (JMUH)

- $\quad$ On route 675, 3.7 miles east of junction of route 11 and Edinburg.; Devonian shale-barren slope.; 4/6/1982; J. Beer (JMUH)

- $\quad 3$ miles north of Kings crossing on route 678.; Shale hill.; 4/25/1983; T. Rexroad 1220 (JMUH)

- Approx. 2 mi. N.W. of the Junction of \#675 and \#678 on \#675; on the Massanutten Mountain; Shale barrens.; 5/4/1977; R.C. Simpson (LFCC)

- Approx. 5 mile East of Edinburg then South of \#678 on a shale barren on the mountain south of the road; Elev. 2300 ft.; 7/7/1978; R.C. Simpson (LFCC)

Anthemis arvensis L. (corn chamomile) *

- Rt. \#11 at Junction with Cedar Creek; Growing on creek bank; Elev. 594 ft.; 5/22/1978;

K. Halstead (LFCC)

Arctium minus Bernh. (lesser burdock)*

- $\quad$ Rt. 706 roadside. $~ 1 \mathrm{~m}$ tall.; 6/30/2011; 38.81697,-78.65918; Domangue 395

Arnoglossum atriplicifolium (L.) H. Rob. (pale Indian plantain)

- $\quad$ Laurel Run Road; 1/2 mile west after intersection with Rt 691, on North side of road; Roadside.; 7/7/1980; R.C. Simpson, S. Gwyn (LFCC)

$\diamond$ Arnoglossum reniforme (Hook.) H. Rob. (great Indian plantain)

Artemisia annua L. (sweet sagewort) *

- Growing in Cedar Grove Church parking lot. Strong scent.; 9/15/2011; 38.69416,-78.64754;

Domangue 621 
Artemisia vulgaris L. (common wormwood) *

- $\quad$ Rt. 672 roadside, next to N. Fork Shenandoah River. Cobbly silt soil.; 9/29/2011; 38.84497, -78.53108; Domangue 649

Bidens aristosa (Michx.) Britt. (bearded beggarticks)

- $\quad$ Rt. 717 roadside (Jerome) below shale barrens.; 9/8/2011; 38.85925,-78.72931; Domangue 605

- 2 miles east of Strasburg on Rt \#55. Roadside in front of Signal Knob farm; Roadside, wasteland; Elev. 850 ft.; 10/23/1978; M. Hughes (LFCC)

- 1 mile south of Strasburg on Rt. \#11 on west roadside; roadside; Flowers: yellow.; 9/17/1975; K.D. Ramsey (LFCC)

Bidens bipinnata $\mathrm{L}$. (Spanish needles)

- $\quad$ Rt. 600 roadside.; 8/2/2011; 38.91550,-78.46568; Domangue 515

- $\quad$ End of Rd 739 East off 42 Edinburg; Farm Field.; 10/10/1976; J.S. McCarthy (LFCC)

- $\quad 1 / 4$ mi. W. of Rt. 628 at Cedar Cr.; open meadow; yellow bloom.; Altitude 980 ft.; 9/26/1975; E. Fowlkes (LFCC)

Bidens cernua $\mathrm{L}$. (nodding beggartick)

- $\quad$ Tomahawk Pond Campground, growing in pond.; 9/22/2011; 38.75889,-78.84070; Domangue 638

- $\quad 1 / 4$ mi North of Strasburg behind C.R. Hupp property; Streamside.; 5/12/1975; C.R. Hupp (LFCC)

Bidens comosa (Gray) Wiegand (threelobe beggarticks)

- Powell's Fort Organization Camp (in massanutten mts); Elev. 1500 ft.; 9/12/1978; R.C. Simpson (LFCC)

Bidens frondosa $\mathrm{L}$. (devil's beggartick)

- Strasburg Park, in the middle of grassy field.; 9/15/2011; 38.97712,-78.35555; Domangue 626

Bidens vulgata Greene (big devils beggartick)

- 1 mile E. of Liberty Furnace at junction of Rt \#717 and 690.; Shale barrens.; 9/7/1975; R.C. Simpson (LFCC)

- 1 mile E. of Liberty Furnace at junction of Rt. \#717 and 690.; Flood plain.; 9/7/1975; R.C. Simpson (LFCC)

Carduus acanthoides L. (spiny plumeless thistle) *

- $\quad$ Rt. 11 roadside along fencerow. $1.5 \mathrm{~m}$ tall.; 7/20/2011; 38.66240,-78.66306; Domangue, C.K. McMullen 449

- $\quad$ Rt. 706 roadside. $1 \mathrm{~m}$ tall.; 6/30/2011; 38.81697,-78.65918; Domangue 396

- Beside parking lot of Mt. Jackson feed mill. Growing with purple C. acanthoides and Cirsium vulgare.; 7/26/1972; M. Hensley (JMUH)

- $\quad$ End of Rd 739 East off 42 Edinburg; Farm Field.; 10/10/1976; J.S. McCarthy (LFCC)

Carduus nutans L. (nodding plumeless thistle) *

- Rt. 706 roadside on bank beside cultivated field.; 6/3/2011; 38.81726,-78.65349; Domangue, M. Lantz 302

- $\quad$ Rt. 648 along roadside at Sandy Hook, Strasburg.; 10/22/1975; M.L. Snarr (LFCC) 
Centaurea biebersteinii DC. (spotted knapweed) *

- Fort Valley, growing beside driveway in shale and clay. $1 \mathrm{~m}$ tall.; 6/24/2011; 38.88448, -78.37763; Domangue, J. Courtwright 387

- $\quad$ Rt. 601 So. 1 mi So Stbg; Pappas Farm; Elev. 575.; 9/29/1979; P. Johns (LFCC)

- Strasburg on Rt \#55 at Standard Quarry.; in field.; 6/17/1975; R. Simpson, C. Hupp (LFCC)

$\diamond$ Centaurea solstitialis L. (yellow star-thistle) *

Chondrilla juncea $\mathrm{L}$. (rush skeletonweed)

- $\quad$ Rt. 11 roadside along fencerow.; 7/20/2011; 38.66240,-78.66306; Domangue, C.K. McMullen 446

- $\quad$ Scattered in a pasture.; 9/3/1961; (JMUH)

- Hockman Homestead (Chequers) US Rt. 11 on east side of road 1 mile north of Edinburg.; 7/9/1977; G. Evans (LFCC)

Chrysogonum virginianum $\mathrm{L}$. (green and gold)

- Mooreland Gap Rd., in grassy clearing below power lines.; 4/26/2011; 38.71304,-78.58643;

Domangue, M. Lantz 111

- Mt. Jackson; shale mountain.; 5/1983; J. Kauffman (HAVI)

- Camp Caroline Furnace, Fort Valley (7 miles SE of Edinburg on Route 675). On bank surrounding camp spring.; Grassy, rich soil.; 4/23/1979; B.E. Brandt 29 (JMUH)

- Mudhole Bog by Little Passage Creek in Little Fort Valley. Growing in a boggy area near a stream.; 6/28/1972; BIO 68523 (JMUH)

- Rte. 771 Powell's Fort Camp, Little Fort Valley, Mud Hole Bog.; rich woods, moist bog area.; 7/2/1979; VA Flora Class 10 (JMUH)

- Fort Valley; Dry woods.; 5/16/1964; L. Artz (JMUH)

- Massanutten Mountains (northern); Mudhole Bog.; 7/5/1978; J. Huffman, B. Latham, D. Stoltzfus 21 (JMUH)

- $\quad$ Peters Mill, Little Fort.; 6/20/1975; R.C. Simpson (LFCC)

- Rt. \#678 1 mile south of Elizabeth Furnace; Growing on side of road; Elev. 725 ft.; 5/22/1978;

K. Halstead (LFCC)

$\diamond$ Chrysopsis mariana (L.) Ell. (Maryland goldenaster)

Cichorium intybus L. (chicory)*

- Cedar Grove Church, beside parking lot in weedy patch.; 6/15/2011; 38.69409,-78.64780;

Domangue 327

- 1 mile north of Edinburg on U.S Rt. 11 N. of dwelling; Open field.; 9/28/1975; G. Beaty (LFCC)

- $\quad$ Rt. 648 along roadside at Sandy Hook, Strasburg.; 10/22/1978; M.L. Snarr (LFCC)

$\diamond$ Cirsium altissimum (L.) Hill (tall thistle)

+ Cirsium arvense (L.) Scop. (Canada thistle) *

- $\quad$ Rt. 698 roadside, along old fencerow.; 7/1/2011; 38.81673,-78.57175; Domangue 408

Cirsium discolor (Muhl. ex Willd.) Spreng. (field thistle)

- $\quad$ Rt. 717 roadside (Jerome) below shale barrens. 2 m tall.; 9/8/2011; 38.85925,-78.72931; Domangue 606

- $\quad$ 1/4 mi W. of Rt. 628 at Cedar Creek; fields and roadsides; hairy stem; Altitude 900 ft.; 9/29/1975; E. Fowlkes (LFCC) 
Cirsium muticum Michx. (swamp thistle)

- $\quad$ Mud Hole Gap.; 9/19/1975; R.C. Simpson (LFCC)

- $\quad$ Powels Fort Camp; Bog.; 10/1/1979; T. Leight, R. Simpson, C. Hupp (LFCC)

$\diamond$ Cirsium pumilum (Nutt.) Spreng. (pasture thistle)

Cirsium vulgare (Savi) Ten. (bull thistle) *

- Shenandoah Co. Park, in lightly forested area near rt. 11.; 8/2/2011; 38.93506,-78.45602;

Domangue 517

- $\quad$ Rt. 663 roadside, next to open field.; 8/2/2011; 38.90121,-78.47547; Domangue 514

$\diamond$ Cnicus benedictus $\mathrm{L}$. (blessed thistle) *

Conoclinium coelestinum (L.) DC. (blue mistflower)

- Growing on the N. Fork Shenandoah River floodplain off Black Bear Rd.; 7/20/2011; 38.90966, -78.42236; Domangue, C.K. McMullen 456

- $\quad$ Strasburg Park, in ditch beside parking lot.; 7/12/2011; 38.97360,-78.35113; Domangue 433

- Rt. 551 mile west of Lebanon Church, then north off 55 about 100 yards.; Rich woods; Elev. 1000 ft.; 10/20/1978; M. Hughes (LFCC)

Conyza canadensis (L.) Cronq. (Canadian horseweed)

- $\quad$ Growing off rt. 620, in overgrown grassy lot beside lawn.; 8/16/2011; 38.69393,-78.64685; Domangue 547

- Common weed.; 8/15/1961; (JMUH)

- $\quad$ 1/4 mi. W. of Rt 628 at Cedar Creek.; Open sunny area by path; Altitude 930 ft.; 9/29/1975; E. Fowlkes (LFCC)

Coreopsis lanceolata $\mathrm{L}$. (lanceleaf tickseed)

- $\quad$ Rt. 771 roadside, 0.24 km from rt. 774 intersection. Shale and clay soil.; 5/30/2012; 38.87840, -78.40237; Domangue 708

- $\quad$ Rocky, dry field; Strasburg Quarry, 2nd Dirt road off of Rt 55 W from Strasburg.; Flowers: yellow.; 6/1/1975; K. Ramsey (LFCC)

- 3 mi. north of King's Crossing; Flowers: yellow.; 5/31/1977; R.C. Simpson (LFCC)

$\diamond$ Coreopsis tripteris L. (tall tickseed)

Coreopsis verticillata $\mathrm{L}$. (whorled tickseed)

- $\quad$ Rt. 758 roadside, 0.58 km from Stage Coach Rd. intersection. Shale bank.; 5/30/2012; 38.87424, -78.45996; Domangue 710

- $\quad$ Rte. 771 Powell's Fort Camp, Little Fort Valley, Mud Hole Bog.; dry area, cut forest, along roadside.; 7/2/1979; VA Flora Class 5 (JMUH)

- $\quad .2 \mathrm{mi} \mathrm{S}$ of Natl Forest on Co. Rt. 678 (Fort); shale barrens.; 8/8/1976; C.R. Hupp (LFCC)

- $\quad$ Rt 789.; 5/20/1980; R.C. Simpson (LFCC)

$\diamond$ Crepis capillaris $(\mathrm{L}$.$) Wallr. (smooth hawksbeard) *$

Doellingeria infirma (Michx.) Greene (cornel-leaf whitetop)

- $\quad$ Fort Valley; 5 miles north of Elizabeth Furnace; Elev. 800 ft.; 8/4/1980; R.C. Simpson (LFCC) 
$\diamond$ Doellingeria umbellata (Mill.) Nees (parasol whitetop)

Eclipta prostrata (L.) L. (false daisy)

- $\quad$ Below Meems Bottom Covered Bridge at N. Fork Shenandoah River.; 8/16/2011; 38.72073, -78.65482; Domangue 551

Erechtites hieraciifolia (L.) Raf. ex DC. (American burnweed)

- $\quad$ Strasburg Park, growing in ditch near small stream.; 8/16/2011; 38.97655,-78.35470; Domangue 565

- $\quad$ Scattered in moist soil.; 8/10/1961; (JMUH)

- Shale Barrens 1 mile east of Liberty Furnace at junc. Of Rt. \#717 and \#609.; 9/7/1975; R.C. Simpson (LFCC)

Erigeron anпииs (L.) Pers. (eastern daisy fleabane)

- Shenandoah Co. Park. In grassy field among cedar trees.; 5/30/2011; 38.93316,-78.45297; Domangue 296

- $\quad$ Edinburg; open field; Elev. 800 ft.; 8/25/1978; B. Baker (LFCC)

- Hockman Homestead ("Chequers") 1 mile north of Edinburg on U.S. Route 11 on east side of road; Open field grazed by cattle and unmowed lawn.; 6/13/1980; G. Evans (LFCC)

Erigeron philadelphicus L. (Philadelphia fleabane)

- Shenandoah Co. Park. Edge of dirt trail in lightly forested area.; 5/18/2011; 38.93341,-78.45766; Domangue 229

- Clearing in wooded area near small cabin.; 5/11/2011; 38.94997,-78.52692; Domangue, C.K. McMullen 192

- Elizabeth Furnace Recreation Area on the edge of parking lot.; 5/4/2011; 38.92796,-78.32958; Domangue 149

- Strasburg sidewalk crack Washington St.; 5/3/1975; M. Dyson-Cobb (LFCC)

- $\quad$ G. W. Nat. Forest, Camp Roosevelt, parking lot; Growing on side of road (parking lot.); Elev. 780 ft.; 5/22/1978; K. Halstead (LFCC)

Erigeron pulchellus Michx. (robin's plantain)

- Elizabeth Furnace Recreation Area, growing in lawn near Passage Creek.; 4/19/2012; 38.92847, -78.33035; Domangue 679

- $\quad$ Low, open woods; $1 / 4$ mi N of Strasburg, behind F.R. Hupp property.; 5/15/1975; C.R. Hupp (LFCC)

Erigeron strigosus Muhl. ex Willd. (prairie fleabane)

- In cow pasture of weeds and woody plants.; 6/30/2011; 38.79334,-78.65990; Domangue 399

- In weedy lot at rt. 11 intersection with rt. 620.; 5/30/2011; 38.69481,-78.64817; Domangue 283

Eupatorium altissimum L. (tall thoroughwort)

- $\quad$ Rt. 620 roadside across from Cedar Grove Church. $1 \mathrm{~m}$ tall.; 9/15/2011; 38.69452,-78.64764; Domangue 620

- $\quad$ Tomahawk Pond Campground, in grassy area a few meters from pond. $1 \mathrm{~m}$ tall.; 8/8/2011; 38.75772,-78.84098; Domangue 525

- $\quad$ Conicville, beside dirt trail in woods. $1 \mathrm{~m}$ tall.; 8/2/2011; 38.83074,-78.67409; Domangue 523 
Eupatorium fistulosum Barratt (trumpetweed)

- $\quad$ Rt. 730 roadside at forest border.; 7/29/2011; 38.71855,-78.57798; Domangue 483

- Fishers Hill on Rt. 601; Fishers Hill Dam.; in sandy soil by water's edge.; plants standing 6'+ high.; stem: glabrous, purple and hollow; flowers: purple.; 9/17/1975; K.D. Ramsey (LFCC)

Eupatorium perfoliatum L. (common boneset)

- In cow pasture beside small stream. $1.5 \mathrm{~m}$ tall.; 8/8/2011; 38.79440,-78.66000; Domangue 543

- Fishers Hill on Rt \#601 at the Fishers Hill Dam; growing in sandy soil along the water's edge.; some plants reached a height 6'+. All werebadly eaten by insects.; 9/17/1975; K.D. Ramsey (LFCC)

$\diamond$ Eupatorium pilosum Walt. (rough boneset)

$\diamond$ Eupatorium pubescens Muhl. ex Willd. (roundleaf thoroughwort)

Eupatorium purpureum L. (sweetscented joe pye weed)

- $\quad$ Near Woodstock on Rt \#758, 1/2 way up west side of Massanutten Mts.; 8/9/1975; R.C. Simpson (LFCC)

$\diamond$ Eupatorium rotundifolium L. (roundleaf thoroughwort)

Eupatorium sessilifolium L. (upland boneset)

- $\quad$ Edinburg Gap; Dry woods.; 6/8/1964; L. Artz (JMUH)

Eurybia divaricata (L.) Nesom (white wood aster)

- $\quad$ Rt. 724 roadside near small stream.; 9/22/2011; 38.78112,-78.77615; Domangue 644

- Elizabeth Furnace Recreation Area on dirt trail a few meters from Passage Creek.; 8/23/2011; 38.92903,-78.32871; Domangue 582

- $\quad$ Prescribed burn; Elev. 3000 ft.; 9/5/1980; R.C. Simpson (LFCC)

- 1 mile E. of Liberty Furnace at the junction of Rt. 717 and 690. Stony Creek flood plain.; 9/7/1975; R.C. Simpson (LFCC)

$\diamond$ Eurybia macrophylla (L.) Cass. (bigleaf aster)

$\diamond$ Eurybia schreberi (Nees) Nees (Schreber's aster)

Euthamia graminifolia (L.) Nutt. (flat-top goldentop)

- $\quad$ Growing beside gravel lot in forest off rt. 691. $1 \mathrm{~m}$ tall.; 9/8/2011; 38.89235,-78.70585; Domangue 618

- $\quad$ Rt. 717 roadside below steep shale bank.; 8/8/2011; 38.85930,-78.72937; Domangue 538

$\diamond$ Filago vulgaris Lam. (common cottonrose) *

Galinsoga parviflora Cav. (gallant soldier) *

- $\quad$ 1/4 mi W. of Rt. 628 at Cedar Cr.; Creek bank.; white bloom w. yellow center; Altitude 800 ft.; 9/29/1975; E. Fowlkes (LFCC)

- $\quad$ End of Rd. 739 East off Rte 42 Edinburg; Farm Field.; 10/10/1976; J.S. McCarthy (LFCC) 
Galinsoga quadriradiata Ruiz \& Pavon (shaggy soldier) *

- Growing on the N. Fork Shenandoah River floodplain off Black Bear Rd.; 7/20/2011; 38.90966, -78.42236; Domangue, C.K. McMullen 455

- $\quad$ Bear Wallow Trail.; 8/2/1978; S.M. Collins (LFCC)

Gamochaeta purpurea (L.) Cabrera (spoonleaf purple everlasting)

- $\quad$ Duncan Hollow; Dry soil; Altitude 1250.; 7/21/1965; L. Artz (JMUH)

- $\quad$ Little Fort Recreation trail.; 6/13/1980; R.C. Simpson (LFCC)

$\diamond$ Helenium autumnale L. var. autumnale (common sneezeweed)

$\diamond$ Helenium flexuosum Raf. (purplehead sneezeweed)

Helianthus decapetalus L. (thinleaf sunflower)

- In moist soil of a steep woodland slope.; 8/25/1961; (JMUH)

Helianthus divaricatus L. (woodland sunflower)

- $\quad$ Rt. 730 roadside at forest border. $1 \mathrm{~m}$ tall.; 7/29/2011; 38.71311,-78.58607; Domangue 478

- Rt. 730 roadside in forest clearing. $1.5 \mathrm{~m}$ tall.; 6/24/2011; 38.71222,-78.58757; Domangue, J. Courtwright 354

- Common wayside plant in dry slaty soil.; 8/10/1961; (JMUH)

$\diamond$ Helianthus laevigatus Torr. \& Gray (smooth sunflower)

Helianthus strumosus L. (paleleaf woodland sunflower)

- $\quad$ Fort Valley; 5 miles north of Elizabeth Furnace; Elev. 800 ft.; 8/4/1980; R.C. Simpson (LFCC)

- $\quad$ Elizabeth Furnace; Shale Barrens.; 7/13/1979; R.C. Simpson (LFCC)

Helianthus tuberosus L. (Jerusalem artichoke)

- $\quad$ Strasburg Park, on the edge of grassy field. $1.5 \mathrm{~m}$ tall. Lower leaves opposite.; 9/15/2011; 38.97509,-78.35458; Domangue 630

- $\quad$ Elizabeth Furnace Recreation Area, beside Passage Creek. $1.5 \mathrm{~m}$ tall.; 7/29/2011; 38.92918, -78.32920; Domangue 491

- In low ground; Conicville area.; 8/15/1961; (JMUH)

Heliopsis helianthoides (L.) Sweet (smooth oxeye)

- $\quad$ Fort Valley; 5 miles north of Elizabeth Furnace; Elev. 800 ft.; 8/4/1980; R.C. Simpson (LFCC)

Hieracium caespitosum Dumort. (meadow hawkweed) *

- Weedy lot near rt. 11 and rt. 620 intersection. Clay soil.; 5/9/2012; 38.69520,-78.64876; Domangue 698

- Shenandoah Co. Park. Growing among tall grasses at the edge of grass trail.; 5/18/2011; 38.93763,-78.45066; Domangue 230

- $\quad$ FR 274 West.; 6/24/1981; D.E. Miller 51 (JMUH)

Hieracium gronovii $\mathrm{L}$. (queendevil)

- $\quad$ Peters Mill, Little Fort.; 6/20/1975; R.C. Simpson (LFCC)

$\diamond$ Hieracium paniculatum L. (Allegheny hawkweed) 
Hieracium pilosella L. (mouseear hawkweed) *

- Camp Roosevelt at the edge of parking lot.; 5/25/2011; 38.73018,-78.51694; Domangue 257

- $\quad$ Peter Mill Trail.; 6/16/1978; R.C. Simpson (LFCC)

- 6 mi West of Columbia Furnace on Laurel Run at intersection of Rt. \#671 and Rt. \#717; dry barren ground in old apple orchard.; 6/5/1978; R. Simpson (LFCC)

$\diamond$ Hieracium scabrum Michx. var. scabrum (rough hawkweed)

$\diamond$ Hieracium traillii Greene (Maryland hawkweed)

Hieracium venosum $\mathrm{L}$. (rattlesnakeweed)

- Woodstock, Riverview Park beside Effinger Trail.; 5/18/2011; 38.87241,-78.49030; Domangue 226, 227

- $\quad$ Woodstock, Riverview Park on edge of Effinger Trail.; 5/18/2011; 38.87173,-78.49010; Domangue 223

- Growing in the middle of dirt road in forest.; 5/11/2011; 38.94842,-78.52805; Domangue, C.K. McMullen 205

- Devil's Backbone State Forest on the edge of dirt road in forest. Clay soil.; 5/10/2011; 39.04796, -78.43324; Domangue, B. Burkholder 177

- Deciduous woods; Fishers Hill, 2 mi SW of Strasburg, woods just west of Fishers Hill UCC church.; Flowers: yellow.; 6/7/1975; K. Ramsey (LFCC)

- $\quad$ Rt 789.; 5/20/1980; R.C. Simpson (LFCC)

$\diamond$ Ionactis linariifolius (L.) Greene (flaxleaf whitetop aster)

Krigia virginica (L.) Willd. (Virginia dwarfdandelion)

- Mt. Jackson; shale mountain.; 5/1983; J. Kauffman (HAVI)

Lactuca canadensis L. (Canada lettuce)

- $\quad$ Rt. 730 roadside at the edge of woods. Flowers yellow, drying blue.; 8/23/2011; 38.71303,78.58588; Domangue 571

Lactuca floridana (L.) Gaertn. (woodland lettuce)

- Elizabeth Furnace Recreation Area, on the edge of parking lot. $2 \mathrm{~m}$ tall.; 7/29/2011; 38.92960, -78.32800; Domangue 493

$\diamond$ Lactuca saligna $\mathrm{L}$. (willowleaf lettuce) *

Lactuca serriola L. (prickly lettuce) *

- $\quad$ Fort Valley, growing beside gravel drive. Flowers yellow, drying blue.; 7/29/2011; 38.88442, -78.37753; Domangue 496

- Valley View Orchard along Route 622 west of Strasburg; Open ground in apple orchard; Elev. 800'.; 8/25/1978; B. Rinker (LFCC)

- Hockman Homestead (Chequers) US Rt. 11 on east side of road 1 mi. north of Edinburg; unused garden.; 7/9/1977; G. Evans (LFCC)

+ Lapsana communis L. (common nipplewort) *

- $\quad$ Roadside, at rt. 11 and rt. 672 intersection.; 6/15/2011; 38.84656,-78.53180; Domangue 342 
Leucanthemum vulgare Lam. (oxeye daisy) *

- In weedy lot at rt. 11 intersection with rt. 620.; 5/30/2011; 38.69481,-78.64817; Domangue 281

- $\quad$ Story Trail, off FR 274 West.; 6/24/1981; D.E. Miller 54 (JMUH)

- Hockman Homestead ("Chequers") 1 mile north of Edinburg on U.S. Route 11 on east side of Road; Unmowed lawn, grasses.; 6/13/1980; G. Evans (LFCC)

- Hockman Homestead. One mile north of Edinburg on U.S. Rt 11. East of dwelling; Open field.; 10/25/1975; G. Beaty (LFCC)

Liatris scariosa (L.) Willd. (devil's bite)

- $\quad$ 1/2 mile south of Wolf Gap on \#675; wooded roadside.; 9/7/1975; R.C. Simpson (LFCC)

- $\quad$ Prescribed burn; Elev. 3000 ft.; 9/5/1980; R.C. Simpson (LFCC)

Matricaria discoidea DC. (disc mayweed) *

- Growing in weedy pasture area once used for livestock. Sweet fragrance when crushed.; 5/4/2011; 38.87190,-78.57690; Domangue 156

- $\quad 3.0$ miles NE of Lebanon Church on Rt. 628.; 5/21/1988; Chandler 4 (JMUH)

- $\quad$ Rt 691, Burn site; Road side; Elev. 3,005 feet.; 7/7/1980; S. Gwyn, R.C. Simpson (LFCC)

- Devils Hole Mountains, Rt 691 on South side of road; Roadside; Elev. 3,005 feet.; 7/7/1980; S. Gwyn, R.C. Simpson (LFCC)

$\diamond$ Oclemena acuminata (Michx.) Greene (whorled wood aster)

$\diamond$ Packera anonyma (Wood) W.A. Weber \& Á. Löve (Small's ragwort)

Packera antennariifolia (Britton) W.A. Weber \& Á. Löve (shale barren ragwort)

- $\quad$ Fort Valley, North of Detrick on rt. 678; Shale barren.; 5/17/1989; C. Jacobs 32 (JMUH)

- $\quad 0.5$ miles from Catherine's Furnace on a shale bank; shale bank endemic on Devonian shales.; 4/14/1980; Blake 94 (JMUH)

- 3.7 mi, E on 675 past Edinburg; shale barren.; 5/18/1983; BIO 50197 (JMUH)

- $\quad$ Road side bank; Ft. Valley Geo. Wash. Nat. Forest west bank.; Flowers: yellow.; 4/20/1975; R.C. Simpson (LFCC)

- $\quad$ Fort Valley, 5.7 miles south on Route 678 from Junction with Route 55.; Shale; Elev. 1000 feet.; 4/25/1980; L.R. Stewart (LFCC)

Packera aurea (L.) Á. \& D. Löve (golden ragwort)

- $\quad$ Little Fort Valley, Little Fort Valley road; Roadside meadow.; 5/17/1989; C. Jacobs 34 (JMUH)

- Common along streams.; 7/29/1963; (JMUH)

- Rt \#691, Bear Oak Prescribed Burn, near Va. - W. Va. state line; along roadbank of unburned area; Elev. 3005 feet.; 5/21/1981; S. Collins (LFCC)

- $\quad$ Fishers Hill Dam 2 mi South of Strasburg growing along damn's west bank.; 4/30/1975; K. Ramsey (LFCC) 
Packera obovata (Muhl. ex Willd.) W.A. Weber \& Á. Löve (roundleaf ragwort)

- Tomahawk Pond Campground, growing in lawn near parking lot in woods.; 3/29/2012; 38.75812, -78.84177; Domangue 668

- $\quad$ Fort Valley, growing beside Mine Mountain Rd. on bank.; 4/26/2011; 38.89637,-78.40578;

Domangue, M. Lantz 120

- Camp Caroline Furnace, Fort Valley (7 miles SE of Edinburg, on Route 675). Camp dump.; Dry roadbank.; 4/23/1979; B.E. Brandt 35 (JMUH)

- $\quad$ Roadside bank; Ft. Valley George Wash. Nat. Forest. West bank.; Flowers; yellow.; 4/20/1975; R.C. Simpson (LFCC)

- Near Pugh's Run. On Rt. 663, .5 miles East of junction of Rt. 11 and Rt. 663; on top of limestone cliffs, shallow lateritic soil; Elev. 720 ft.; 4/29/1978; R.C. Simpson (LFCC)

Packera plattensis (Nutt.) W.A. Weber \& Á. Löve (prairie groundsel)

- Strasburg, Junction of Rt 55 and Rt 623; Dry, grassy field; Elev. 700 ft.; 5/23/1978; R.F. Whiting (LFCC)

$\diamond$ Parthenium auriculatum Britt. (wild quinine)

Parthenium integrifolium L. (wild quinine)

- Rt. 675 (Wolf Gap) roadside at forest border.; 8/8/2011; 38.91578,-78.69146; Domangue 534

- $\quad$ FR 274 east.; 7/22/1981; D.E. Miller 9 (JMUH)

- Massanutten Mtn. Little Fort Recreation area, along Powell's Mountain trail (to Edinburg gap) about 1 mi. south of the Little Fort Camping area; Along woodland Jeep trail; Elev. 1390.; 6/26/1977; R.C. Simpson, G. Evans (LFCC)

- $\quad$ Peters Mill, Little Fort.; 6/20/1975; R.C. Simpson (LFCC)

$\diamond$ Polymnia canadensis L. (whiteflower leafcup)

$\diamond$ Prenanthes alba $\mathrm{L}$. (white rattlesnakeroot)

$\diamond$ Prenanthes altissima $\mathrm{L}$. (tall rattlesnakeroot)

$\diamond$ Prenanthes serpentaria Pursh (cankerweed)

$\diamond$ Pseudognaphalium helleri (Britt.) Anderb. var. micradenum (Weath.) Kartesz (Heller's cudweed)

Pseudognaphalium obtusifolium (L.) Hilliard \& Burtt (rabbit-tobacco)

- $\quad$ Buzzard rocks trail, in old field to east of trail.; old field, bordering woods; Elev. 800.; 10/7/1980; W. Ludtice (LFCC)

- 1 mile E. of Liberty Furnace at junction of 717 and 690.; 9/7/1975; R.C. Simpson (LFCC)

$\diamond$ Rudbeckia fulgida Ait. (orange coneflower) 
Rudbeckia hirta L. (blackeyed Susan)

- $\quad$ Rt. 675 roadside in weedy area near Stony Creek. 1 m tall.; 8/8/2011; 38.86973,-78.63084; Domangue 533

- $\quad$ Fort Valley, growing in open, grassy field.; 7/29/2011; 38.88439,-78.37679; Domangue 495

- $\quad$ FR 374 roadside. Silty soil.; 6/24/2011; 38.72910,-78.56737; Domangue, J. Courtwright 362

- $\quad$ Scattered in a hay field. Not a common species.; 8/4/1961; (JMUH)

- Peters Mill, Little Fort.; 6/20/1975; R.C. Simpson (LFCC)

- 1 mi. So Stbg Rt 601 So.; Pappas Farm; Elev. 575.; 9/29/1979; P. Johns (LFCC)

Rudbeckia laciniata L. (cutleaf coneflower)

- $\quad$ Rt. 717 roadside (Jerome) below shale barrens. $1 \mathrm{~m}$ tall.; 9/8/2011; 38.85925,-78.72931; Domangue 607

- $\quad$ Powell's Fort Camp, George Washington National Forest; moist, rich ground, thicket.; 8/21/1978; S.M. Collins (LFCC)

Rudbeckia triloba L. (browneyed Susan)

- $\quad$ Along Route 622 near Valley View Orchard, Strasburg; Open roadside; Elev. 800'.; 8/25/1978;

B. Rinker (LFCC)

- 2 miles east of Strasburg on Rt. \#55. Roadside in front of Signal Knob farm; waste lands roadsides; Elev. 850.; 11/3/1978; M. Hughes (LFCC)

+ Senecio vulgaris $\mathrm{L}$. (old-man-in-the-Spring) *

- Shenandoah Co. Park. Growing in grassy lawn.; 4/18/2011; 38.93561,-78.45190; Domangue, J. Courtwright 104

Sericocarpus asteroides (L.) B.S.P. (toothed whitetop aster)

- $\quad$ Edinburg Gap shale barren, westside; Shale.; 7/13/1975; R.C. Simpson (LFCC)

$\diamond$ Sericocarpus linifolius (L.) B.S.P. (narrowleaf whitetop aster)

Silphium asteriscus L. (starry rosinweed)

- $\quad$ Kings Crossing, Massanutten.; 6/26/1975; R.C. Simpson (LFCC)

- Massanutten Mountain; Fort Valley on \#678 at shale barrens around road to glass house.; 6/26/1977; R.C. Simpson (LFCC)

Smallanthus uvedalia (L.) Mack. ex Small (hairy leafcup)

- Strasburg Park, in weedy patch next to open field.; 7/12/2011; 38.97570,-78.35461; Domangue 437

Solidago altissima L. (Canada goldenrod)

- $\quad 2$ miles East of Strasburg on Rt. 55. 100 yards from Shenandoah River; open field; Elev. 850 ft.; 10/26/1978; M. Hughes (LFCC)

Solidago arguta Ait. (Atlantic goldenrod)

- Massanutten Mountain, Fort Valley on \#678 at shale barrens around road to glass house; open shale barrens.; 6/26/1977; R.C. Simpson, D. Steed (LFCC)

- $\quad$ Fort Valley, north end of Rt. 678 shale barrens.; 8/11/1975; R.C. Simpson (LFCC) 
Solidago bicolor L. (white goldenrod)

- Tomahawk Pond Campground, on the edge of pond.; 9/22/2011; 38.75898,-78.84066; Domangue 637

- $\quad$ Mud Hole Gap.; 9/19/1975; R.C. Simpson (LFCC)

Solidago caesia $\mathrm{L}$. (wreath goldenrod)

- $\quad$ Rt. 724 roadside near small stream.; 9/22/2011; 38.78112,-78.77615; Domangue 642, 643

- In oak woods.; 8/1/1963; (JMUH)

- Powell's Fort Organization Camp (in massanutten mts); Elev. 1500 ft.; 9/12/1978; R.C. Simpson (LFCC)

- 1 mile E. of Liberty Furnace at junctions 717 and 690, Stony Creek flood plain.; 9/7/1975; R.C. Simpson (LFCC)

Solidago canadensis $\mathrm{L}$. (Canada goldenrod)

- $\quad$ Fishers Hill Dam; Wet Meadow.; 9/5/1975; R.C. Simpson (LFCC)

- Rt. 608 at Columbia Furnace along roadside which leads to Wolf Gap.; 10/19/1978; M.L. Snarr (LFCC)

$\diamond$ Solidago curtisii Torr. \& Gray var. curtisii (mountain decumbent goldenrod)

Solidago erecta Pursh (showy goldenrod)

- 1 mile E. of Liberty Furnace at junction of Rt. 717 and 690.; Shale barren.; 9/7/1975; R.C. Simpson (LFCC)

$\diamond$ Solidago flexicaulis L. (zigzag goldenrod)

+ Solidago gigantea Ait. (giant goldenrod)

- $\quad$ Rt. 672 roadside in weedy area.; 9/1/2011; 38.84562,-78.53024; Domangue 595

Solidago harrisii Steele (Harris' goldenrod)

- Woodstock, Riverview Park, off Effinger Trail in woods.; 7/12/2011; 38.87204,-78.49036;

Domangue 429

Solidago hispida Muhl. ex Willd. (hairy goldenrod)

- Prescribed burn; Elev. 3000 ft.; 9/5/1980; R.C. Simpson (LFCC)

Solidago juncea Ait. (early goldenrod)

- $\quad$ FR 274 West.; 7/30/1981; D.E. Miller 72 (JMUH)

- $\quad$ Fort Valley 1 mile north of Detrick; Elev. 940 ft.; 7/7/1978; R.C. Simpson (LFCC)

- $\quad$ Fort Valley, Rt 678, westside; Shale.; 7/13/1975; R. Simpson (LFCC)

Solidago nemoralis Ait. (gray goldenrod)

- $\quad$ Prescribed burn; Elev. 3000 ft.; 9/5/1980; R.C. Simpson (LFCC)

- Fort Valley, (North end) Rt \#758, mile 1/2 west of Little Fort Rec. area.; 8/9/1975; R.C. Simpson (LFCC)

+ Solidago patula Muhl. ex Willd. (roundleaf goldenrod)

- $\quad$ In low ground.; 9/29/1962; (JMUH) 
$\diamond$ Solidago puberula Nutt. (downy goldenrod)

$\diamond$ Solidago rigida L. ssp. rigida (stiff goldenrod)

Solidago roanensis Porter (Roan Mountain goldenrod)

- Conicville, beside dirt trail in woods.; 8/2/2011; 38.83195,-78.67377; Domangue 521, 522

$\diamond$ Solidago rugosa Mill. (wrinkleleaf goldenrod)

$\diamond$ Solidago squarrosa Nutt. (stout goldenrod)

Solidago ulmifolia Muhl. ex Willd. (elmleaf goldenrod)

- $\quad$ Shenandoah Co. Park, on the edge of woods near rt. 11.; 9/1/2011; 38.93426,-78.45569;

Domangue 597

- $\quad$ Rt. 730 roadside at forest border.; 7/29/2011; 38.71311,-78.58607; Domangue 479

- In moist soil along Gap Creek.; 8/10/1961; (JMUH)

- Tom's Brook; Roadside.; 9/9/1980; R.C. Simpson (LFCC)

- $\quad$ Fort Valley (North end) Rt 758, 1/2 mile west of Little Fort Rec. are.; shale barren.; 8/9/1975; R.C. Simpson (LFCC)

+ Sonchus arvensis L. (field sowthistle) *

- $\quad$ Roadside, at the intersection of rt. 717 and rt. 611.; 9/8/2011; 38.87644,-78.71243; Domangue 611

Sonchus asper (L.) Hill (spiny sowthistle) *

- Weedy lot near rt. 11 and rt. 620 intersection. Hollow stem.; 5/9/2012; 38.69489,-78.64821;

Domangue 699

$\diamond$ Sonchus oleraceus L. (common sowthistle) *

$\diamond$ Symphyotrichum concolor $($ L.) Nesom (eastern silver aster)

Symphyotrichum cordifolium (L.) Nesom (common blue wood aster)

- $\quad$ 1/4 mi W. of Rt. 628 at Cedar Creek.; Shady - rocky old vegetation; light lavender bloom w. dk. center 2 cm - d., ht. - 1 m.; Altitude 850'.; 9/29/1975; E. Fowlkes (LFCC)

- $\quad 2.5$ miles west of Columbia Furnace at junction of Rt. \#717 and \#675; Shale Barrens.; 9/7/1975; R.C. Simpson (LFCC)

Symphyotrichum dumosum (L.) Nesom (rice button aster)

- Rt. 730 roadside in forest clearing. Flowers pale violet.; 6/24/2011; 38.71222,-78.58757;

Domangue, J. Courtwright 358

Symphyotrichum laeve (L.) Á. \& D. Löve (smooth blue aster)

- $\quad$ Rt. 730 roadside at forest border.; 10/6/2011; 38.71304,-78.58604; Domangue 653

- Rt. 691 roadside bank, 100 m from rt. 705 intersection.; 9/22/2011; 38.85762,-78.68478; Domangue 647 
Symphyotrichum lateriflorum (L.) Á. \& D. Löve (calico aster)

- $\quad$ Powell's Fort Camp.; 10/8/1979; T. Leight, C. Hupp, R.C. Simpson (LFCC)

- Powell's Fort Organization Camp (in massanutten mts); Elev. 1500 ft.; 9/12/1978; R.C. Simpson (LFCC)

$\diamond$ Symphyotrichum lowrieanum (Porter) Nesom (Lowrie's blue wood aster)

$\diamond$ Symphyotrichum novae-angliae (L.) Nesom (New England aster)

Symphyotrichum oblongifolium (Nutt.) Nesom (aromatic aster)

- Woodstock, Riverview Park, on rocky ledge off Effinger Trail.; 9/29/2011; 38.87134,-78.49026; Domangue 650

Symphyotrichum patens (Ait.) Nesom (late purple aster)

- $\quad$ Rt. 730 roadside at forest border. $1 \mathrm{~m}$ tall.; 10/6/2011; 38.71304,-78.58604; Domangue 652

- Rt 6011 mi. So. Stbg; Pappas Farm; Elev. 575 ft.; 9/29/1979; P. Johns (LFCC)

- $\quad$ Rt. 648 along side of road at Sandy Hook, Strasburg.; 10/22/1978; M.L. Snarr (LFCC)

$\diamond$ Symphyotrichum phlogifolium (Muhl. ex Willd.) Nesom (thinleaf late purple aster)

Symphyotrichum pilosum (Willd.) Nesom (hairy white oldfield aster)

- Growing in gravel lot in forest off rt. 691. $1 \mathrm{~m}$ tall.; 9/8/2011; 38.89268,-78.70621; Domangue 617

- $\quad$ Rt. 648 along roadside at Sandy Hook, Strasburg.; 10/22/1978; M.L. Snarr (LFCC)

- junction of Rt. \#717 and \#675 west of Columbia Furnace; Shale Barrens.; 9/7/1975; R.C. Simpson (LFCC)

Symphyotrichum prenanthoides (Muhl. ex Willd.) Nesom (crookedstem aster)

- $\quad 1.0$ mi. up from the George Washington National Forest entrance off of Route 678. Moist, sandy, riparian environment. Species locally abundant. Stems zig-zagging. Leaves clasping stem. Flowers purple.; 9/27/2003; M.J. Carmichael 7 (JMUH)

- $\quad 1 / 4$ mi W. of Rt 628 at Cedar Cr.; open fields and roadsides; Altitude 930'.; 9/30/1975;

E. Fowlkes (LFCC)

$\diamond$ Symphyotrichum puniceum (L.) Á. \& D. Löve var. puniceum (purplestem aster)

Symphyotrichum racemosum (Ell.) Nesom (smooth white oldfield aster)

- $\quad$ Rt. 724 roadside near small stream.; 9/22/2011; 38.78112,-78.77615; Domangue 645

- $\quad$ Tomahawk Pond Campground, a few meters from pond. Clay soil.; 9/22/2011; 38.75877, -78.84037; Domangue 636

Symphyotrichum undulatum (L.) Nesom (wavyleaf aster)

- $\quad$ Prescribed burn; Elev. 3000 ft.; 9/5/1980; R.C. Simpson (LFCC)

- Powell's Fort Recreation Camp.; 9/23/1978; R.C. Simpson (LFCC)

+ Tanacetum vulgare $\mathrm{L}$. (common tansy) *

- $\quad$ 1/4 mi. W. of Rt. 628 at Cedar Creek; against building foundation; flat topped yellow bloom clusters; Altitude 930 ft.; 9/29/1975; E. Fowlkes (LFCC) 
Taraxacum officinale G.H. Weber ex Wiggers (common dandelion) *

- Shenandoah Co. Park. In lawn directly adjacent to tennis courts.; 4/5/2011; 38.93472,-78.45238;

Domangue 80

- Home yard, Mt. Jackson; Cultivated.; 6/1/1987; Jennifer Estep (BDWR)

- Hockman Homestead 1 mile north of Edinburg U.S. Rt. 1110 ft. N. of dwelling; lawn.; 10/2/1975; G. Beaty (LFCC)

- $\quad$ Rt \#691, Bear Oak Prescribed Burn, near Va. - W. Va. line; along roadbank of burned side; Flower - yellow; Elev. 3005 feet.; 4/26/1981; S.M. Collins (LFCC)

Tragopogon dubius Scop. (yellow salsify) *

- On rt. 620 roadside across from Cedar Grove Church.; 5/18/2011; 38.69433,-78.64742;

Domangue 211

- $\quad$ Fort Valley, North of Detrick on rt. 678; Roadside bank.; 5/17/1989; C. Jacobs 33 (JMUH)

- $\quad$ Rt. \#11 at Junction with I-81; Growing along roadside; Elev. 600 ft.; 5/22/1975; K. Halstead (LFCC)

- Hockman Homestead ("Chequers") 1 mile north of Edinburg on U.S. Route 11 on east side of road; Unmowed lawn.; 6/13/1950; G. Evans (LFCC)

Tragopogon porrifolius L. (salsify) *

- $\quad$ Rt. 767 roadside ditch near Forestville. $1 \mathrm{~m}$ tall.; 5/9/2012; 38.71370,-78.72142; Domangue 695

- $\quad 1 / 4$ mi. south of Rt. 11 and I-81 at Strasburg.; 5/22/1979; R.C. Simpson (LFCC)

- Road to Woodstock Tower; flower yellow.; 5/26/1980; S.M. Collins, J.A. Hepner (LFCC)

$\diamond$ Tragopogon pratensis L. (Jack-go-to-bed-at-noon) *

Tussilago farfara L. (coltsfoot) *

- $\quad$ Tomahawk Pond Campground. Edge of lower parking lot.; 4/11/2011; 38.75972,-78.84129; Domangue 82

- Camp Caroline Furnace, Fort Valley (7 miles S.E. of Edinburg on Route 675); west bank of Passage Creek, 100 yards south of Ridge Trail Bridge.; Rocky, sandy flood plain.; 4/3/1979; B.E. Brandt 4 (JMUH)

- Fort Valley, Powell's Fort Camp; roadside.; 3/13/1977; R.C. Simpson (LFCC)

- Rt \#691, Bear Oak Prescribed Burn, near Va. - W. Va. state line; along roadbank of unburned side; Flower - yellow; Elev. 3005 feet.; 4/18/1981; S.M. Collins (LFCC)

Verbesina alternifolia (L.) Britt. ex Kearney (wingstem)

- $\quad$ Rt. 717 roadside (Jerome) below shale barrens. $1 \mathrm{~m}$ tall.; 9/8/2011; 38.85925,-78.72931; Domangue 608

- Growing in weedy lot at intersection of rt. 11 and rt. 620. $2 \mathrm{~m}$ tall.; 7/20/2011; 38.69469, -78.64819; Domangue, C.K. McMullen 450

- $\quad$ Fort Valley Rt \#678, 1 mile S. of "Blue hole"; wet roadside area.; 8/9/1975; R.C. Simpson (LFCC)

- $\quad$ 1/4 mi. W. of Rt. 628 at Cedar Cr.; Creek bank; yellow bloom split-covered green stem; Altitude 780 ft.; 9/30/1975; E. Fowlkes (LFCC)

Verbesina occidentalis (L.) Walt. (yellow crownbeard)

- Conicville, growing in forest off rt. 694. $1 \mathrm{~m}$ tall.; 9/22/2011; 38.83003,-78.67470; Domangue 648 
Vernonia glauca (L.) Willd. (broadleaf ironweed)

- Shenandoah Co. Park, beside grassy path in lightly wooded area.; 8/16/2011; 38.93762,-78.45095; Domangue 564

- $\quad$ Rt. 11 roadside along fencerow. 1.5 m tall.; 7/20/2011; 38.66240,-78.66306; Domangue, C.K. McMullen 448

Vernonia noveboracensis (L.) Michx. (New York ironweed)

- $\quad$ Rt. 717 roadside below steep shale bank. $1.5 \mathrm{~m}$ tall.; 8/8/2011; 38.85930,-78.72937; Domangue 537

- In a moist meadow.; 8/20/1961; (JMUH)

- Fort Valley; 5 miles north of Elizabeth Furnace; Elev. 800 ft.; 8/4/1980; R.C. Simpson (LFCC)

Xanthium strumarium L. (rough cocklebur)

- $\quad$ Rt. 672 roadside, a few meters from N. Fork Shenandoah River.; 9/1/2011; 38.84491,-78.53140; Domangue 591

- Common weed in cultivated and waste ground.; 9/16/1961; (JMUH)

\section{Balsaminaceae}

Impatiens capensis Meerb. (jewelweed)

- Elizabeth Furnace Recreation Area, beside grassy field at forest border.; 6/24/2011; 38.92759, -78.32863; Domangue, J. Courtwright 370

- Elizabeth Furnace Picnic Area George Washington National Forest; wet, shady woods.; 8/21/1978; S.M. Collins (LFCC)

- $\quad$ Approx. 50 feet from the river off U.S. route 55; wet, shady places.; 10/18/1978; M. Hughes (LFCC)

$\diamond$ Impatiens pallida Nutt. (pale touch-me-not)

\section{Berberidaceae}

Berberis thunbergii DC. (Japanese barberry)*

- In cow pasture at the corner of rt. 703 and rt. 711. Low shrub.; 8/8/2011; 38.79313,-78.65961; Domangue 544

- $\quad$ Intersection Rt 11 So 627 W Middletown; 3.5 mi; left .2 mi; Alongside driveway; Elev. 760.; 9/29/1979; P. Johns (LFCC)

$\diamond$ Caulophyllum thalictroides (L.) Michx. (blue cohosh)

Jeffersonia diphylla (L.) Pers. (twinleaf)

- Along south side of hwy 648 approx $1 / 4$ east of junction 648 and 11.; north facing slope in woods.; 4/15/1978; M. Kelly (JMUH)

- $\quad$ I-81 $30 \mathrm{mi} \mathrm{N}$ of Harrisonburg to North Fork of the Shenandoah bridge. 100 yards from I-81 on wooded hillside 30 yards above the water's edge.; Rich, moist soil. Wooded area.; 4/14/1992;

A. Logan 15 (JMUH)

- N. facing wooded slope South of Strasburg on Wildflower Hill; white flower, rich moist oakhickory forest containing abundant: dicentra, spicebush.; 4/1/1975; M. Bowles, K. Clarke, D. Smith, G. Smith (LFCC) 
Podophyllum peltatum L. (mayapple)

- Elizabeth Furnace Recreation Area in shady location a few meters from Passage Creek.; 5/4/2011; 38.92952,-78.32879; Domangue 154

- $\quad$ Power line 300 yds. E. of 717; 4 mi N.E. of Basye; creekside, shaded.; 5/21/1984; S. Arey 23 (JMUH)

- $\quad$ Fort Valley, 2.5 mile south from junction of \#55 and \#678 on \#678.; 4/25/1980; T. McCracken (LFCC)

- Powells Fort Camp, George Washington National Forest, 2.6 miles north of the intersection of Rt. 273 and Rt. 66 on Rt. 66.; Broadleaf deciduous forest, seepage area; Elev. 1200 ft.; 4/22/1978; L. Frey (LFCC)

\section{Betulaceae}

Alnus serrulata (Ait.) Willd. (hazel alder)

- $\quad$ Tomahawk Pond Campground, growing on the edge of pond. Tree, $~ 2 \mathrm{~m}$ tall.; 7/13/2011; 38.75891,-78.84059; Domangue 441

- Junction Turkey Run, Rt. 55; Wet roadside ditch.; 10/16/1975; Dendrology Class (LFCC)

$\diamond$ Betula alleghaniensis Britt. (yellow birch)

Betula lenta L. (sweet birch)

- $\quad 1.5$ mi. up from the George Washington National Forest entrance off of Route 678. Steep cliff slope trailside. Predominantly oak, pine, birch, and rhododendron in surrounding forest. Tree was $5 \mathrm{ft}$. tall with strongly aromatic bark that was dark brown, smooth, and shiny.; 9/27/2003; M.J. Carmichael 4 (JMUH)

- $\quad$ Story Trail, off Fr 274, near parking lot.; 6/24/1981; D.E. Miller 65 (JMUH)

- $\quad$ Middle Mt.; Woods; Altitude 1000-2000 feet.; 8/8/1966; M. Krouse (LFCC)

Carpinus caroliniana Walt. (American hornbeam)

- $\quad$ Elizabeth Furnace Recreation Area on edge of parking lot.; 5/25/2011; 38.92780,-78.32964; Domangue 261

- Cedar Creek Wayside, Rt. 114 mi. N. of Strasburg; Flood plain.; 10/2/1975; R. Brown, R. Crowder (LFCC)

- George Washington Nat'l Forest, Elizabeth Furnace Rec. Area, on Rt 678, 1.5 miles South of junc. With Rt. 613; floodplain; Elev. 800 ft.; 10/7/1978; R.F. Whiting (LFCC)

$\diamond$ Corylus americana Walt. (American hazelnut)

Corylus cornuta Marsh. (beaked hazelnut)

- $\quad$ At Little Fort on the Massanutten.; 6/19/1975; R.C. Simpson (LFCC) 
Ostrya virginiana (P.Mill.) K.Koch (hophornbeam)

- Woodstock, Riverview Park beside Effinger Trail. Tree.; 5/30/2011; 38.87160,-78.49010; Domangue 291

- $\quad 3.5$ miles from Edinburg on the right side of Rt. 675 opposite the Shale Barrens.; Rich open woods on slopes and ridges.; 6/22/1977; P. Graves 6 (JMUH)

- Shale barrens; 3.5 miles east from Edinburg along Rt. 675.; 6/27/1978; J. Huffman, B. Latham, D. Stoltzfus 1 (JMUH)

- $\quad$ Paddy's Run, 4 mi W of Star Tannery off of Rt 55; moist mountain stream area; Elev. 900 ft.; 9/9/1978; J. Macdonell (LFCC)

- $\quad$ East of Strasburg on Route 678, north end of Massanutten Mt. in Fort Valley at Elizabeth Furnace Campground.; Growing in understory of oak hickory forest.; Elev. 800'.; 10/3/1977; O. Bate (LFCC)

\section{Bignoniaceae}

Campsis radicans (L.) Seem. ex Bureau (trumpet creeper)

- $\quad$ Rt. 11 roadside, along weedy fencerow.; 7/1/2011; 38.93569,-78.45542; Domangue 414

- $\quad$ Edinburg Gap.; 7/28/1975; R. \& H. Simpson (LFCC)

+ Catalpa bignonioides Walt. (southern catalpa)*

- $\quad$ Large tree in cow pasture next to stream.; 6/30/2011; 38.79452,-78.65999; Domangue 403

- $\quad$ Town of Edinburg Main Street; Growing in yard.; 7/1/1977; R.F. Lytton 10 (JMUH)

$\diamond$ Catalpa speciosa (Warder) Warder ex Engelm. (northern catalpa)*

\section{Boraginaceae}

Buglossoides arvensis (L.) I.M. Johnst. (corn gromwell) *

- $\quad$ Fort Valley, in open field among dense grasses.; 4/26/2011; 38.88433,-78.37682; Domangue, M. Lantz 132

- Rt. 730 roadside, next to agriculture field.; 3/21/2011; 38.71165,-78.64343; Domangue, J. Courtwright 62

- $\quad$ Rt. 675 Fort Valley.; 5/21/1986; BIO 501 (JMUH)

- Near Elizabeth Furnace S; Shale Barrens.; 4/25/1980; R.C. Simpson (LFCC)

- Near Strasburg, junct. Rt. 11 and I81 on Frontage Road; mustard field.; 4/17/1977; (LFCC)

Cynoglossum officinale L. (gypsyflower) *

- $\quad 3.0$ miles NE of Lebanon Church on Rt. 628.; 5/21/1988; Chandler 13 (JMUH)

- $\quad$ North-west side of slope; Roadside ditch on Woodstock Mountain; Flower pink-red.; 5/20/1975; M.T. Bowles (LFCC)

Cynoglossum virginianum L. (wild comfrey)

- Devil's Backbone State Forest on the edge of dirt road in forest. Clay soil.; 5/10/2011; 39.04685, -78.42977; Domangue, B. Burkholder 174

- North of Tom's brook on \#651 about .5 mi West of \#11 (between \#11 and \#81); Old overgrown woodland trail.; 5/4/1977; R.C. Simpson (LFCC)

- $\quad$ Laurel Run.; 5/27/1980; R.C. Simpson (LFCC) 
Echium vulgare L. (common viper's bugloss) *

- Shenandoah Co. Park. In grassy field among cedar trees.; 5/30/2011; 38.93287,-78.45461;

Domangue 297

- $\quad$ FR 274 East.; 7/22/1981; D.E. Miller 25 (JMUH)

- $\quad 2$ miles east of Strasburg on Rt. \#55. 100 yds from Shenandoah River; open field; Elev. 850 ft.; 10/31/1978; M. Hughes (LFCC)

- Strasburg on Rt \#55 at Standard Quarry; growing under a power cut.; 6/17/1975; R. Simpson, C. Hupp (LFCC)

$\diamond$ Hackelia virginiana (L.) I.M. Johnst. (beggarslice)

Lithospermum canescens (Michx.) Lehm. (hoary puccoon)

- Near Pugh's Run. On Rt. 663, .5 miles East of junction of Rt. 11 and Rt. 663; on top of limestone cliffs, shallow lateritic soil; Elev. 720 ft.; 4/29/1978; R.C. Simpson (LFCC)

- Fishers Hill Dam; Fisher hill, 2 mi. south of Strasburg. Growing on a rocky south facing slope.; 4/30/1975; K. Ramsey (LFCC)

Mertensia virginica (L.) Pers. ex Link (Virginia bluebells)

- Meems Bottom Covered Bridge near N. Fork Shenandoah River, 0.5 miles west on Wissler Road.; 4/18/2011; 38.72092,-78.65472; Domangue, J. Courtwright 87

- $\quad$ Rich woods, moist meadows; 4.5 miles north of Kings Crossing on Rt. 678.; 4/25/1983; B. Shirey (JMUH)

- 1 mile north of Woodstock on west side of U.S. 11; Streambank.; 4/24/1982; E. Leavy 30 (JMUH)

- On North bank of Stoney Creek about 150-200 yards on the South side of Stoney Creek Rd., 1-1.5 miles East of the Rt. 42 junction (Larkin's Grocery), approximately 1/4 miles downstream from swinging bridge.; 4/13/2001; I. Poplar-Jeffers 1 (JMUH)

- 2.5 miles south of Detrick on Rt. 678; Rich floodplain.; 4/25/1983; M. Daniel 100 (JMUH)

- $\quad 3.0$ miles north of Kings Crossing on Rt. 678 along riverbank; Flood Plain.; 4/25/1983; B. Kelley 29 (JMUH)

- $\quad$ Rt 744 posey hollow Rd, Palmer's property on Shenandoah River; south facing slope; Blue flower; flood plain.; 4/1/1975; M. Bowles, G. Smith, D. Smith, K. Clarke (LFCC)

- Rt. 623 .1 mile north from the intersection of Rt. 623 and Rt. 714.; Roadside in a semi-open area near a stream.; Elev. 600 ft.; 4/24/1978; L. Frey, J. Brumback, R. Whiting (LFCC)

$\diamond$ Myosotis scorpioides L. (true forget-me-not) *

+ Myosotis verna Nutt. (spring forget-me-not)

- Clearing in wooded area near small cabin.; 5/11/2011; 38.94997,-78.52692; Domangue, C.K. McMullen 195

$\diamond$ Onosmodium hispidissimum Mack. (softhair marbleseed) 
Brassicaceae

Alliaria petiolata (M. Bieb.) Cavara \& Grande (garlic mustard) *

- $\quad$ Fort Valley along roadside, adjacent a grassy field.; 5/4/2011; 38.88413,-78.37695; Domangue 145

- Hockman Homestead ("Chequers") 1 mile north of Edinburg on U.S. Route 11 on east side of road; Wooded river bank grazed by cattle.; 6/13/1980; G. Evans (LFCC)

Alyssum alyssoides (L.) L. (pale madwort) *

- Fort Valley. Growing at the edge of a gravel lot in clay soil.; 5/4/2011; 38.88472,-78.37834; Domangue 142

- $\quad$ Fields, waste places, shale.; 4 mi. north of Kings Crossing, Fort Valley Rt. 678.; 4/25/1983;

B. Shirey (JMUH)

Arabidopsis thaliana (L.) Heynh. (mouseear cress) *

- $\quad$ Near Strasburg; junct. Rt. 11 and I81 on Frontage Road; mustard field; Elev. 640 ft.; 4/17/1977; (LFCC)

$\diamond$ Arabis canadensis L. (sicklepod)

$\diamond$ Arabis hirsuta (L.) Scop. var. adpressipilis (M. Hopkins) Rollins (creamflower rockcress)

Arabis laevigata (Muhl. ex Willd.) Poir. (smooth rockcress)

- $\quad$ Shale Bank.; Fort Valley, R \#678, 1.5 mi. S. of junction of R \#55 and R \#678. west side of road.; 4/20/1975; R.C. Simpson (LFCC)

Arabis laevigata (Muhl. ex Willd.) Poir. var. laevigata (smooth rockcress)

- Growing on steep bank beside dirt road. Clay soil.; 4/18/2011; 39.04831,-78.43584; Domangue, J. Courtwright 99

Arabis laevigata (Muhl. ex Willd.) Poir. (smooth rockcress)

- Massanutten Mountain, on the Southern edge of the George Washington Natural Forest; shale barren.; 4/1/1977; R.C. Simpson, C. Hupp (LFCC)

$\diamond$ Arabis lyrata L. (lyrate rockcress)

$\diamond$ Arabis patens Sull. (spreading rockcress)

$\diamond$ Arabis serotina Steele (shale barren rockcress)

Barbarea verna (Mill.) Asch. (early yellowrocket) *

- New Market, .25 mi. south of Junction Rt. \#11 and Rt. \#737 on Rt. \#737; open field along roadside, west side of road; Elev. 1000 Ft.; 5/2/1978; R. Whiting, L. Frey, J. Brumback (LFCC)

- Steep bank of Shenandoah R.; (2 mi. S. of Strasburg) 1 mi. E. of Fishers Hill on Capt. F. Palmers property on bank of Shenandoah River.; 4/15/1975; K. Ramsey (LFCC) 
Barbarea vulgaris R. Brown (garden yellowrocket) *

- Fort Valley, growing among grasses in open field. $1 \mathrm{~m}$ tall.; 5/4/2011; 38.88437,-78.37630; Domangue 147

- Rt \#691, Bear Oak Prescribed Burn, near Va. - W. Va. state line; along roadside of unburned area; Elev. 3005 feet.; 5/16/1981; S.M. Collins (LFCC)

$\diamond$ Brassica juncea (L.) Czern. (brown mustard) *

$\diamond$ Brassica nigra (L.) W.D. Koch (black mustard) *

Brassica rapa $\mathrm{L}$. (field mustard) *

- $\quad$ Tom's Brook. Beside highway in ditch. $1 \mathrm{~m}$ tall.; 4/18/2011; 38.95588,-78.42992; Domangue, J. Courtwright 100

$\diamond$ Camelina microcarpa Andrz. ex DC. (littlepod false flax)*

+ Capsella bursa-pastoris (L.) Medic. (shepherd's purse) *

- $\quad$ Growing in weedy pasture area once used for livestock.; 5/4/2011; 38.87190,-78.57690; Domangue 157

- Rt. 42 at southern corporate limit of Conicville, VA; Open pasture.; 4/13/1985; M.L. Verts 210 (JMUH)

- George Washington National Forest; Woodstock Tower; Edible parts: Young leaves can be eaten raw or cooked. Seeds can be eaten raw or dried and used as a seasoning.; 5/17/1988; Gordon, Cason 12 (JMUH)

Cardamine bulbosa (Schreb. ex Muhl.) Britton, Sterns \& Poggenb. (bulbous bittercress)

- Camp Caroline Furnace, Fort Valley, (7 miles SE of Edinburg on Route 675). Lake Trail, 50 feet from lake.; Moist, swampy, grassy.; 4/23/1979; B.E. Brandt 27 (JMUH)

- George Washington National Forest - Little Fort Recreation Area; wooded with open spots; white flowers; Elev. 928.; 5/30/1981; E. Lerch (LFCC)

Cardamine concatenata (Michx.) Sw. (cutleaf toothwort)

- Woodstock, Riverview Park. On edge of Effinger Trail, among heavy leaf litter.; 3/29/2011; 38.87266,-78.49052; Domangue 71

- Camp Caroline Furnace, Fort Valley (7 miles S.E. of Edinburg on Route 675); cliffs above west bank of Passage Creek, 100 yards north of Cabin Bridge.; Dry, sunny, shale cliffs.; 4/10/1979; B.E. Brandt 8 (JMUH)

- $\quad 1.2$ miles east from the north fork of the Shenandoah River; rich woods.; 4/14/1982; C. Yost 19 (JMUH)

- $\quad 1.2$ miles east of north fork of Shenandoah River on route 758.; dry soil on slope of woods.; 4/6/1982; J. Beer (JMUH)

- Rt. 623, .7 mile north from the intersection of Rt. 623 and Rt. 714.; Broadleaf, deciduous forest.; Elev. 700 ft.; 4/24/1978; L. Frey, J. Brumback, R. Whiting (LFCC) 
Cardamine hirsuta L. (hairy bittercress) *

- On North bank of Stoney Creek about 150-200 yards on the South side of Stoney Creek Rd., 1-1.5 miles East of the Rt. 42 junction (Larkin's Grocery), approximately 1/4 miles downstream from swinging bridge.; 4/13/2001; I. Poplar-Jeffers 2 (JMUH)

- Massanutten Mountain, north end of A. U. Natl. Forest; Shale barrens.; 4/1/1977; R.C. Simpson, C. Hupp (LFCC)

$\diamond$ Cardamine parviflora L. var. arenicola (Britton) O.E. Schulz (sand bittercress)

Cardamine pensylvanica Muhl. ex Willd. (Pennsylvania bittercress)

- Off rt. 720 a few meters from N. Fork Shenandoah River. Silty soil.; 3/22/2012; 38.72074, -78.65485; Domangue 663

- Mt. Jackson Park on western end of Wunder St.; 3/21/2011; 38.75010,-78.64727; Domangue, J. Courtwright 64

$\diamond$ Descurainia pinnata (Walter) Britton ssp. brachycarpa (Richardson) Detling (western tansymustard)

Draba ramosissima Desv. (branched draba)

- Woodstock, Riverview Park on Effinger Trail. Creeping stem. Dark soil.; 5/18/2011; 38.87173, -78.49010; Domangue 222

- $\quad$ Intersection of 776 and 775.; 4/19/1977; W.B. Clark 39 (JMUH)

- Rt 675 Fort Valley Edinburg; calcareous rocky banks and shale barrens.; 5/21/1986; BIO 501 (JMUH)

- $\quad 3.0$ miles north of Kings Crossing on Rt. 678 on rocky shale bank along roadside; Calcareous rocky banks and shale barrens.; 4/25/1983; B. Kelley 14 (JMUH)

- $\quad$ Fort Valley; Shale barrens.; 4/25/1980; (LFCC)

- $\quad$ Fort Valley on \#678 at Seven Fountains.; 4/17/1977; R.C. Simpson, J. Bursey (LFCC)

Draba verna L. (spring draba) *

- Woodstock, Riverview Park. Near Effinger Trail entrance, growing through dense mosses.; 3/29/2011; 38.87339,-78.49115; Domangue 76

- $1 \mathrm{ml}$ from Rt 42 on Rt 675 along stony crk; Damp field.; 4/9/1977; C.R. Hupp (LFCC)

Hesperis matronalis L. (dames rocket) *

- Public boat landing at N. Fork Shenandoah River. 1 m tall.; 5/18/2011; 38.84583,-78.52956; Domangue 214

- $\quad$ Fort Valley area.; 5/18/1984; BIO 501134 (JMUH)

- $\quad$ Approximately 2 miles North of Woodstock off US Rt 11 at Pugh's Run; shaded, weedy stream bank; Elev. 800 ft.; 6/5/1977; R. Simpson, K. Ramsey (LFCC)

- Orkney Springs Area; Junction Routes 614 and 273; Roadside; Elev. 1868.; 5/22/1981; E. Lerch (LFCC)

$\diamond$ Isatis tinctoria L. (Dyer's woad) *

+ Leavenworthia uniflora (Michx.) Britton (Michaux's gladecress)

- Rt. 733 roadside, on the edge of Flat Rock Church parking lot.; 3/22/2012; 38.69979,-78.74548; Domangue 666 
Lepidium campestre (L.) R. Brown (field pepperweed) *

- Tomahawk Pond Campground, in clearing next to pond. Clay soil.; 5/10/2011; 38.75791, -78.84087; Domangue, B. Burkholder 162

- Strasburg, Junction of Rt 55 and Rt 623.; Dry, grassy field; Elev. 700 ft.; 5/23/1978; R.F. Whiting (LFCC)

- Fishers Hill Dam; Fishers Hill, 2 mi. south of Strasburg. Growing along side of road that leads to the Dam.; 4/30/1975; K. Ramsey (LFCC)

Lepidium virginicum $\mathrm{L}$. (Virginia pepperweed)

- $\quad$ Woodstock, Riverview Park on Effinger Trail. Rocky soil.; 5/18/2011; 38.87214,-78.49666; Domangue 221

- Valley View Orchard west of Strasburg on Route 622; open ground in apple orchard; Elev. 800'.; 8/22/1978; B. Rinker (LFCC)

+ Lunaria annua L. (annual honesty) *

- $\quad$ Fort Valley, beside Ramsey Rd. on steep bank above creek.; 4/26/2011; 38.89257,-78.37066; Domangue, M. Lantz 129

Microthlaspi perfoliatum (L.) F.K. Mey. (claspleaf pennycress) *

- Woodstock, Riverview Park. On steep slope in gravelly clay soil. Dense population.; 3/29/2011; 38.87439,-78.49010; Domangue 74

- 5.7 mile from junction of Rt 55 and Rt 678 on \#678; Shale barren.; 4/25/1980; T. McCracken (LFCC)

- $\quad$ North facing slope South of Strasburg on Wildflower Hill; rich moist oak-hickory forest; white flower.; 4/1/1975; M. Bowles, K. Clarke, D. Smith, G. Smith (LFCC)

Nasturtium officinale R. Brown (watercress) *

- Fort Valley, beside Mine Mountain Rd. in small stream in woods.; 4/26/2011; 38.89643, -78.40716; Domangue, M. Lantz 124

- Stream at Mine Mt. \& Cove Forest Powells Ft. Rd.; stream.; 3/27/1977; R. Simpson (LFCC)

Raphanus raphanistrum $\mathrm{L}$. (wild radish) *

- $\quad$ Strasburg, Junction of Rt. 55 and Rt. 623; Dry, grassy field; Elev. 700 ft.; 5/23/1978; R.F. Whiting (LFCC)

+ Rorippa sylvestris (L.) Besser (creeping yellowcress) *

- Woodstock, Riverview Park, growing next to N. Fork Shenandoah River.; 7/1/2011; 38.87077, -78.48998; Domangue 411

$\diamond$ Sisymbrium altissimum L. (tall tumblemustard) *

Sisymbrium officinale (L.) Scop. (hedgemustard) *

- Shenandoah Co. Park. Edge of grass trail in light forest area. $1 \mathrm{~m}$ tall.; 5/18/2011; 38.93680, -78.45241; Domangue 235

- $\quad$ Growing in weedy pasture area once used for livestock.; 5/4/2011; 38.87190,-78.57690; Domangue 158 
Thlaspi arvense L. (field pennycress) *

- $\quad$ Growing in weedy pasture area once used for livestock.; 5/4/2011; 38.87190,-78.57690;

Domangue 159

Cactaceae

Opuntia humifusa (Raf.) Raf. (devil's-tongue)

- $\quad$ Fort Valley, growing beside driveway in shale and clay.; 6/24/2011; 38.88448,-78.37763;

Domangue, J. Courtwright 386

- $\quad$ Dry soils, shale barrens; Rt. 678, Fort Valley.; 5/21/1986; (JMUH)

Callitrichaceae

Callitriche heterophylla Pursh (twoheaded water-starwort)

- $\quad$ Rt. 685, 1.3 mi N Rt. 675 (NW Edinburg); Elev. 850 ft.; 6/19/1977; G. Evans, K. Ramsey, R.C. Simpson (LFCC)

Campanulaceae

$\diamond$ Campanula aparinoides Pursh (marsh bellflower)

$\diamond$ Campanula divaricata Michx. (small bonny bellflower)

+ Campanula rapunculoides $\mathrm{L}$. (rampion bellflower) *

- Woodstock, Riverview Park, beside parking lot in weedy area.; 6/15/2011; 38.87435,-78.49007;

Domangue 346

Campanulastrum americanum (L.) Small (American bellflower)

- Rt. 730 roadside in forest clearing. Plant with milky sap, $\sim 1.5 \mathrm{~m}$ tall.; 6/24/2011; 38.71222, -78.58757; Domangue, J. Courtwright 355

Lobelia cardinalis L. (cardinalflower)

- In cow pasture beside small stream. $1 \mathrm{~m}$ tall.; 8/8/2011; 38.79458,-78.66023; Domangue 540

- Ft. Valley entrance just beyond 1st George Washington Forest sign.; flowers: brilliant deep red.; 7/28/1975; R. \& H. Simpson (LFCC)

- $\quad$ Powell's Fort Camp, George Washington National Forest; wet streambank.; 8/21/1978; S.M. Collins (LFCC)

Lobelia inflata L. (Indian-tobacco)

- Camp Roosevelt, growing in shaded, moss-covered soil.; 7/29/2011; 38.72942,-78.51696; Domangue 488

- $\quad$ Strasburg Park, growing beside trail a few meters from N. Fork Shenandoah River.; 7/12/2011; 38.97363,-78.35220; Domangue 434

- $\quad$ FR 274 East.; 7/22/1981; D.E. Miller 27 (JMUH)

- $\quad$ Massanutten Mt.; dry open field \& thickets; Altitude 800 feet.; 8/15/1966; M. Krouse (LFCC)

- $\quad$ Prescribed burn; Elev. 3000 ft.; 9/5/1980; R.C. Simpson (LFCC) 
Lobelia siphilitica L. (great blue lobelia)

- In cow pasture beside small stream. $1 \mathrm{~m}$ tall.; 8/8/2011; 38.79458,-78.66023; Domangue 541

- $\quad$ Little Fort Valley; Stream bank in bog; Altitude 1100 ft.; 9/13/1963; L. Artz (JMUH)

- 2 miles east of Strasburg on Rt. \#55; $50 \mathrm{ft}$. from the Shenandoah River; Creek bed, dried up; Elev. 850.; 10/18/1978; M.J. Hughes (LFCC)

- $\quad$ Fishers Hill on Rt \#601, Fishers Hill Dam on Tumbling Run Creek; stream bank side; flowers: light purple.; 9/5/1975; K.D. Ramsey (LFCC)

Lobelia spicata Lam. (palespike lobelia)

- Devil's Backbone State Forest in grassy clearing.; 6/8/2011; 39.04882,-78.43182; Domangue 320

- Massanutten Mt., Little Fort Recreation Area, along Powell's Mt. Trail (to Edinburg Gap) about 1 mi. south of the Little Fort Camping Area; Note: Flowers white; Elev. 1390 ft.; 6/26/1977; R.C. Simpson, G. Evans, D. Steed (LFCC)

- Ft. Valley, Little Ft. Recreation area.; Flowers = pale lavender.; 6/19/1975; R.C. Simpson (LFCC)

Triodanis perfoliata (L.) Nieuwl. (clasping Venus' looking-glass)

- Devil's Backbone State Forest. Growing beside forest road.; 6/8/2011; 39.04684,-78.42990; Domangue 315

- Woodstock, Riverview Park. Found on grassy trail in shade.; 5/30/2011; 38.87308,-78.48867; Domangue 285

\section{Cannabaceae}

Humulus japonicus Sieb. \& Zucc. (Japanese hop)*

- Below Meems Bottom Covered Bridge at N. Fork Shenandoah River.; 8/16/2011; 38.72073, -78.65482; Domangue 548

$\diamond$ Humulus lupulus L. (common hop)

\section{Caprifoliaceae}

$\diamond$ Lonicera canadensis Bartr. ex Marsh. (American fly honeysuckle)

$\diamond$ Lonicera dioica L. var. dioica (limber honeysuckle)

Lonicera japonica Thunb. (Japanese honeysuckle) *

- Rt. 620 roadside near intersection with rt. 11.; 10/27/2011; 38.69457,-78.64842; Domangue 660

- $\quad$ Fort Valley, in open weedy lot. Dense vines.; 5/25/2011; 38.88446,-78.37760; Domangue 272

- $\quad$ George Washington National Forest; Woodstock Tower; growing along side road.; Elev. 1500.; 10/14/1979; J. Evans (LFCC)

- $\quad$ 1/4 mi W. of Rt. 628 at Cedar Cr.; field and roadsides on fences; hairy leaves; Altitude $850 \mathrm{ft}$.; 10/15/1975; E. Fowlkes (LFCC)

+ Lonicera maackii (Rupr.) Maximowicz (Amur honeysuckle) *

- $\quad$ Lightly forested area at the edge of dirt road. Small shrub.; 5/11/2011; 38.94395,-78.52020;

Domangue, C.K. McMullen 207 
+ Lonicera morrowii Gray (Morrow's honeysuckle) *

- $\quad$ On rt. 712 roadside along fencerow. Low, spreading shrub.; 4/11/2012; 38.76944,-78.66015; Domangue 669

+ Lonicera $x$ bella Zabel (showy fly honeysuckle)

- Woodstock, on S Water St. roadside. Small shrub, 2 m tall.; 4/11/2012; 38.86882,-78.51104; Domangue 673

$\diamond$ Symphoricarpos albus (L.) Blake (common snowberry)

Symphoricarpos orbiculatus Moench (coralberry)

- $\quad$ Shenandoah Co. Park, in overgrown, grassy field.; 7/12/2011; 38.93623,-78.45219; Domangue 430

Symphoricarpos orbiculatus Moench (coralberry)

- Near bridge at Elizabeth Furnace Campground, east side 10 yds from Passage Creek.; disturbed area beside road.; 11/6/1980; W. Ludtice (LFCC)

$\diamond$ Triosteum angustifolium $\mathrm{L}$. (yellowfruit horse-gentian)

$\diamond$ Triosteum perfoliatum L. (feverwort)

\section{Caryophyllaceae}

Arenaria serpyllifolia $\mathrm{L}$. (thymeleaf sandwort) *

- Fort Valley. Growing in a weedy, gravel lot.; 5/4/2011; 38.88472,-78.37834; Domangue 141

- Strasburg, Junction of Rt 55 and Rt 623.; Dry, grassy field; Elev. 700 ft.; 5/23/1978; R.F. Whiting (LFCC)

Cerastium fontanum Baumg. ssp. vulgare (Hartm.) Greuter \& Burdet (big chickweed) *

- Clearing in wooded area near small cabin.; 5/11/2011; 38.94997,-78.52692; Domangue, C.K. McMullen 193

Cerastium glomeratum Thuill. (sticky chickweed) *

- Fisher Hill Dam; Fisher Hill, 2 mi. south of Strasburg. Growing on west bank, half submerged in the H20.; 5/2/1975; K. Ramsey (LFCC)

- Near Strasburg; junct Rt. 11 and I81 on Frontage Road; mustard field; Elev. 640 ft.; 4/17/1977; (LFCC)

Cerastium glomeratum Thuill. (sticky chickweed) *

- $\quad$ Rt. 733 roadside, on the edge of Flat Rock Church parking lot.; 3/22/2012; 38.69979,-78.74548; Domangue 667

$\diamond$ Cerastium nutans Raf. var. nutans (nodding chickweed) 
Dianthus armeria L. (Deptford pink) *

- In weedy lot at rt. 11 intersection with rt. 620.; 5/30/2011; 38.69481,-78.64817; Domangue 282

- Shale barrens; 3.5 miles east from Edinburg along Rt. 675.; 6/27/1978; J. Huffman, B. Latham, D. Stoltzfus 4 (JMUH)

- Valley View Orchard on Route 622 west of Strasburg; Open ground in apple orchard; Elev. 800'.; 8/25/1978; B. Rinker (LFCC)

- Grassy bank on side of road; Fishers Hill, 2 mi. S.W. of Strasburg.; Flowers: pink with white tiny dots on petals.; 6/7/1975; K. Ramsey (LFCC)

Holosteum umbellatum L. (jagged chickweed) *

- Shenandoah Co. Park. In grass lawn. Leaves succulent.; 4/18/2011; 38.93520,-78.45329;

Domangue, J. Courtwright 101

$\diamond$ Minuartia michauxii (Fenzl) Farw. var. michauxii (Michaux's stitchwort)

+ Minuartia patula (Michx.) Mattf. (pitcher's stitchwort)

- Flat Rock Church. Growing in gravel parking lot among larger rocks.; 5/20/2011; 38.69972, -78.74601; Domangue 244

Paronychia canadensis (L.) Wood (smooth forked nailwort)

- $\quad$ Scothern Gap Trail, Massanutten; Dry soil.; 7/29/1965; L. Artz (LFCC)

Paronychia fastigiata (Raf.) Fern. (hairy forked nailwort)

- $\quad$ Rt 678, at "Glass house."; 7/13/1975; R.C. Simpson (LFCC)

- 2.5 mile W. of Columbia Furnace at junction of Rt. 717 and 690.; 9/7/1975; R.C. Simpson (LFCC)

Paronychia montana (Small) Pax \& K. Hoffm. (mountain nailwort)

- Woodstock, Riverview Park, at Effinger Trail. Shale.; 6/15/2011; 38.87204,-78.49020; Domangue 345

- $\quad$ Scothern Gap; Dry woods; Altitude 1400.; 7/21/1965; L. Artz (JMUH)

Petrorhagia prolifera (L.) P.W. Ball \& Heywood (childing pink) *

- Fort Valley, in heavily grazed cow pasture.; 6/24/2011; 38.88457,-78.37936; Domangue, J. Courtwright 385

- $\quad$ Fort Valley near M. Krause property.; 8/11/1978; M. Krouse (LFCC)

Saponaria officinalis L. (bouncingbet) *

- $\quad$ Rt. 706 roadside.; 6/30/2011; 38.81697,-78.65918; Domangue 394

- Hockman Homestead 1 mile north of Edinburg. U.S. Rt 11. $100 \mathrm{ft}$. SSW of dwelling; Garden Plot.; 10/2/1975; G. Beaty (LFCC)

- Hockman Homestead 1 mi. north of Edinburg US Rt. 11 on east side of road.; Elev. 850 ft.; 6/20/1977; G. Evans (LFCC)

$\diamond$ Scleranthus annuus L. (German knotgrass) *

$\diamond$ Silene antirrhina L. (sleepy silene) 
Silene caroliniana Walt. var. pensylvanica (Michx.) Fernald (sticky catchfly)

- Clearing in wooded area near small cabin.; 5/11/2011; 38.94997,-78.52692; Domangue, C.K. McMullen 186

- Camp Roosevelt, along horse trail in wooded area.; 4/26/2011; 38.73071,-78.51150; Domangue, M. Lantz 113

- Growing on steep bank beside dirt road. Clay soil.; 4/18/2011; 39.04831,-78.43584; Domangue, J. Courtwright 98

- Fishers Hill Dam; Fishers Hill, 2 mi. south of Strasburg on a south facing rocky slope.; 4/30/1975; K. Ramsey (LFCC)

- $\quad$ Rt \#691, Bear Oak Prescribed Burn, near Va. - W. Va. line; along roadbank of burned area; Elev. 3005 feet.; 5/9/1981; S.M. Collins (LFCC)

$\diamond$ Silene dichotoma Ehrh. (forked catchfly)*

Silene latifolia Poir. (bladder campion) *

- $\quad$ Splinters Ln. roadside, in shady area along fencerow. $1 \mathrm{~m}$ tall.; 6/15/2011; 38.69614,-78.61810; Domangue 337

- Rt. 716 roadside on grassy bank along fencerow.; 5/20/2011; 38.76545,-78.71974; Domangue 242

- $\quad$ On side of Wissler Road near Meems Bottom Covered Bridge.; 4/26/2011; 38.72066,-78.65475; Domangue, M. Lantz 133

- On the Massanutten Mountain, on the Woodstock Tower road.; 5/4/1975; R.C. Simpson (LFCC)

- Strasburg.; 5/16/1977; L. Little (LFCC)

Silene stellata (L.) Ait. f. (widowsfrill)

- $\quad$ Rt. 730 roadside at the edge of woods near FR 374.; 8/23/2011; 38.72857,-78.56722; Domangue 573

- $\quad$ FR 274 east.; 7/8/1981; D.E. Miller 39 (JMUH)

- Massanutten Mountain, Fort Valley on \#678 at shale barrens around road to glass house; shale barrens.; 6/26/1977; R.C. Simpson, G. Evans (LFCC)

$\diamond$ Silene virginica $\mathrm{L}$. (fire pink)

Silene vulgaris (Moench) Garcke (maidenstears) *

- $\quad$ Rt. 620 roadside, along old fencerow.; 6/15/2011; 38.69464,-78.64809; Domangue 336

Stellaria media (L.) Vill. (common chickweed) *

- Shenandoah Co. Park. Growing in grassy lawn.; 4/18/2011; 38.93485,-78.45294; Domangue, J. Courtwright 102

- 1 mi. on south side of Rt 711 (off of Rt 42) Conicville, VA.; Open field.; 4/13/1985; M.L. Verts 140 (JMUH)

- $\quad$ Shale Cliffs and River Bank at Leisure Point on Tower Rd.; 4/27/1977; R. Simpson (LFCC)

- N. facing, wooded slope; South of Strasburg on Wildflower Hill, white flower, rich moist oakhickory forest with dicentra and spicebush.; 4/1/1975; M. Bowles, K. Clarke, D. Smith, G. Smith (LFCC) 
Stellaria pubera Michx. (star chickweed)

- Camp Roosevelt, along horse trail in wooded area.; 4/26/2011; 38.72899,-78.51307; Domangue, M. Lantz 115

- George Washington National Forest, Rt. 6781.7 miles north of the Day Use Area at Elizabeth Furnace.; Broadleaf, deciduous forest, Oak-hickory, roadside; Elev. 875 ft.; 5/16/1978; L. Frey, R. Whiting, J. Brumback (LFCC)

\section{Celastraceae}

$\diamond$ Celastrus scandens $\mathrm{L}$. (American bittersweet)

+ Euonymus alatus (Thunb.) Sieb. (burningbush) *

- $\quad$ Elizabeth Furnace Recreation Area on the edge of parking lot.; 5/4/2011; 38.92743,-78.32964; Domangue 148

$\diamond$ Euonymus atropurpureus Jacq. var. atropurpureus (eastern wahoo)

+ Euonymus fortunei (Turcz.) Hand.-Maz. (winter creeper) *

- On bank above N. Fork Shenandoah River floodplain off Black Bear Rd. Climbing vine.; 7/20/2011; 38.90966,-78.42236; Domangue, C.K. McMullen 460

$\diamond$ Paxistima canbyi A. Gray (Canby's mountain-lover)

Chenopodiaceae

Chenopodium album L. (lambsquarters) *

- $\quad$ Rt. 720 roadside near Meems Bottom Covered Bridge.; 9/15/2011; 38.72065,-78.65437; Domangue 624

- Hockman Homestead (Chequers) 1 mile north of Edinburg; US Rt. 11 on east side of road; garden.; 7/9/1977; G. Evans (LFCC)

Chenopodium ambrosioides L. (Mexican tea) *

- Massanutten Academy Farm Woodstock; Meadow near river.; 10/17/1976; J.S. McCarthy (LFCC)

$\diamond$ Chenopodium foggii Wahl (Fogg's goosefoot)

Chenopodium standleyanum Aellen (Standley's goosefoot)

- Woodstock, Riverview Park, in lightly forested area.; 8/16/2011; 38.87284,-78.48879; Domangue 557

\section{Cistaceae}

$\diamond$ Helianthemum canadense (L.) Michx. (longbranch frostweed)

$\diamond$ Lechea pulchella Raf. (Leggett's pinweed)

$\diamond$ Lechea racemulosa Michx. (Illinois pinweed) 


\section{Clusiaceae}

$\diamond$ Hypericum canadense L. (lesser Canadian St. Johnswort)

Hypericum gentianoides (L.) B.S.P. (orangegrass)

- $\quad$ Powell's Fort Recreation Camp.; 9/23/1978; R.C. Simpson (LFCC)

Hypericum hypericoides (L.) Crantz (St. Andrew's cross)

- Devil's Backbone State Forest, in the middle of dirt road.; 7/20/2011; 39.04805,-78.43275;

Domangue, C.K. McMullen 463

- Bear Wallow Trail; open woods; yellow-flower.; 8/2/1978; S.M. Collins (LFCC)

Hypericum mutilum L. (dwarf St. Johnswort)

- Fort Valley, 5 miles north of Elizabeth Furnace; Elev. 800 ft.; 8/4/1980; R.C. Simpson (LFCC)

- Rt. 655, 1.3 mi N from Rt 675 NW of Edinburg; Elev. 820 ft.; 6/19/1977; G. Evans, K. Ramsey, R.C. Simpson (LFCC)

Hypericum perforatum L. (common St. Johnswort) *

- $\quad$ Rt. 706 roadside beside cultivated field.; 6/3/2011; 38.81726,-78.65349; Domangue, M. Lantz 304

- Woodstock; Turn off Rt. 11 and go 1 1/4 miles East on Lakeview Road and take left on old dirt road and go 3/4 miles; River bottom meadow.; 7/5/1982; J. Nutter 11 (JMUH)

- $\quad$ FR 274 east.; 7/22/1981; D.E. Miller 10 (JMUH)

- W. of Strasburg on \#622 at Valley View Orchard; dry roadside.; 8/28/1978; B. Rinker (LFCC)

Hypericum prolificum L. (shrubby St. Johnswort)

- $\quad$ Beside Cedar Creek Rd. at forest border.; 7/20/2011; 39.06314,-78.43451; Domangue, C.K. McMullen 461

Hypericum punctatum Lam. (spotted St. Johnswort)

- In cow pasture of weeds and woody plants.; 6/30/2011; 38.79441,-78.66053; Domangue 401

- Dry field, abandoned parking lot.; Fishers Hill, 2 mi. S.W. of Strasburg.; Flowers: yellow, black dots on petal's margin.; 6/7/1975; K. Ramsey (LFCC)

Convolvulaceae

$\diamond$ Calystegia sepium (L.) R. Br. (hedge false bindweed)

Calystegia spithamaea (L.) Pursh (low false bindweed)

- $\quad$ Endemic shale barrens.; 675, Fort Valley.; 5/21/1986; (JMUH)

- $\quad$ Approx 5 mi East of Edinburg then S. of \#678 on a shale barren South of road; Elev. 2300.; 7/7/1978; R.C. Simpson (LFCC)

- On Route 675, approximately 3 miles east of Edinburg; Shale barrens; Associates, yellow buckwheat; Flowers: white.; 5/31/1977; R. Simpson, G. Evans (LFCC)

Convolvulus arvensis $\mathrm{L}$. (field bindweed) *

- Shenandoah Co. Park, in lawn next to parking lot.; 7/1/2011; 38.93528,-78.45226; Domangue 413 
Ipomoea hederacea Jacq. (ivyleaf morning-glory) *

- $\quad$ Rt. 672 roadside near N. Fork Shenandoah River.; 9/1/2011; 38.84521,-78.53076; Domangue 592

- $\quad$ Rt. 601 So - 1 mi So Stbg; Pappas Farm; Elev. 575.; 9/29/1979; P. Johns (LFCC)

- Mill Rd. Woodstock; Roadside.; 9/27/1975; M. Elliott (LFCC)

+ Ipomoea lacunosa L. (whitestar)

- Public boat landing at N. Fork Shenandoah River, a few meters from Narrow Passage Creek.; 8/16/2011; 38.84590,-78.52963; Domangue 552

Ipomoea pandurata (L.) G.F.W. Mey. (man of the earth)

- $\quad$ Woodstock, in ditch next to parking lot.; 7/1/2011; 38.89137,-78.49399; Domangue 412

- $\quad$ Elizabeth Furnace; Shale Barren.; 7/13/1979; R.C. Simpson (LFCC)

- $\quad$ Powells Fort Rd.; 7/25/1976; C.R. Hupp (LFCC)

Ipomoea purpurea (L.) Roth (tall morning-glory) *

- $\quad 1 / 4$ mi W of Rt. 628 at Cedar Creek.; runner on trees and on garden plants.; sunny locations; Altitude 950 ft.; 10/12/1975; E. Fowlkes (LFCC)

Cornaceae

Cornus alternifolia $\mathrm{L}$. (alternateleaf dogwood)

- Ft. Valley at Mud Hole Gap.; 9/19/1975; R.C. Simpson (LFCC)

Cornus aтотит Mill. (silky dogwood)

- 1 mi. E. of Liberty Furnace at junction of 690 and 717; Stoney Creek flood plain.; 9/7/1975; R.C. Simpson (LFCC)

- $\quad$ E end of Edinburg; JCT. Stoney Creek and Shen. River; Elev. 850 ft.; 6/19/1977; G. Evans, K. Ramsey, R.C. Simpson (LFCC)

Cornus florida $\mathrm{L}$. (flowering dogwood)

- Tomahawk Pond Campground. Small tree at the edge of parking lot.; 5/10/2011; 38.75883,78.84087; Domangue, B. Burkholder 168

- $\quad$ Fort Valley, at the edge of open pasture in weedy area near stream. Small tree.; 4/26/2011; 38.88191,-78.37437; Domangue, M. Lantz 119

Cornus florida L. (flowering dogwood)

- $\quad$ Powell's Fort Camp, Mud Hole Bog, on route 771; Found in dry woods along roadside.; 4/19/1977; G. Matthews 52 (JMUH)

- $\quad$ Route 771, Massanutten mountain, near Woodstock tower; Rich mountain-slope.; 4/11/1977; B. Kolankiewicz 31 (JMUH)

- $\quad$ Strasburg, 15 yds W of intersection of A Street and Capon St.; woody area of road.; 10/31/1979; R. Bowden (LFCC)

- East of Strasburg on route 678, north end of Massanutten Mt. in Fort Valley at Elizabeth Furnace Campground.; Growing in understory of oak hickory forest.; Elev. 800'.; 10/3/1977; O. Bate (LFCC) 
Cornus foemina Mill. (stiff dogwood)

- Massanutten Mountain, Little Fort Recreation Area, along Powell's Mountain trail (to Edinburg Gap) about 1 mi. South of the Little Fort camping area; Moist Floodplain.; 6/26/1977; R.C. Simpson, G. Evans, D. Steed (LFCC)

$\diamond$ Cornus racemosa Lam. (gray dogwood)

$\diamond$ Cornus rugosa Lam. (roundleaf dogwood)

\section{Crassulaceae}

Hylotelephium telephioides (Michx.) H. Ohba (Allegheny stonecrop)

- $\quad$ Approx $5 \mathrm{mi}$ E of Edinburg then S. of \#678 on a shale barren South of road; Elev. $2300 \mathrm{ft}$.; 7/7/1978; R.C. Simpson (LFCC)

- 1 mile E. of Liberty Furnace at junction of Rts 717 and 690.; 9/7/1975; R.C. Simpson (LFCC)

$\diamond$ Sedum acre L. (goldmoss stonecrop) *

$\diamond$ Sedum glaucophyllum Clausen (cliff stonecrop)

+ Sedum sarmentosum Bunge (stringy stonecrop) *

- $\quad$ Flat Rock Church. Growing on rocks beside parking lot. Flowers yellow.; 5/20/2011; 38.69972, -78.74601; Domangue 243

Sedum ternatum Michx. (woodland stonecrop)

- Elizabeth Furnace Recreation Area on the edge of parking lot.; 5/4/2011; 38.92870,-78.32921;

Domangue 150

- Rt. 717, Rock Cliff, west side of little wolf hole; fls. white.; 5/27/1980; R.C. Simpson, S. Collins, S. Gwyn (LFCC)

\section{Cucurbitaceae}

Sicyos angulatus $\mathrm{L}$. (oneseed bur cucumber)

- $\quad$ Strasburg Park, on the edge of grassy field.; 9/15/2011; 38.97572,-78.35452; Domangue 629

- $\quad$ Liberty Furnace at junction of Rt's 717 and 690. Stony Creek flood plain.; 9/7/1975; R.C. Simpson (LFCC)

- $\quad$ 1/4 mi W. of Rt 628 at Cedar Creek; partly sunny runner w. tendrils in untrimmed area of yard.; Altitude 980 ft.; 9/29/1975; E. Fowlkes (LFCC)

\section{Cuscutaceae}

$\diamond$ Cuscuta campestris Yunck. (fiveangled dodder)

$\diamond$ Cuscuta gronovii Willd. (scaldweed)

$\diamond$ Cuscuta pentagona Engelm. (fiveangled dodder) 
Diervillaceae

Diervilla lonicera Mill. (northern bush honeysuckle)

- Fort Valley; Peter's Mill Bog.; 6/24/1980; R.C. Simpson (LFCC)

Dipsacaceae

Dipsacus fullonum L. (Fuller's teasel) *

- Rt. 826 roadside, on weedy bank beside cultivated field. $\sim 1 \mathrm{~m}$ tall.; 6/15/2011; 38.69292,

-78.63589; Domangue 338

Droseraceae

Drosera rotundifolia L. (sundew)

- $\quad$ Little Fort V.; Muskeg; Altitude 1400.; 8/14/1965; L. Artz (JMUH)

- Powell's Fort Bog.; 6/8/1980; R.C. Simpson (LFCC)

Ebenaceae

Diospyros virginiana $\mathrm{L}$. (common persimmon)

- $\quad$ Strasburg, Washington St. approx 70 yds W. of low water bridge; wooded area (mixed hardwood); Elev. 650 ft.; 10/13/1979; R. Bowden (LFCC)

Elaeagnaceae

Elaeagnus umbellata Thunb. (autumn olive) *

- Fort Valley, at the edge of open pasture in weedy area near stream. Shrubby tree.; 4/26/2011; 38.88191,-78.37437; Domangue, M. Lantz 118

- $\quad$ At Little Fort on the Massanutten Mt.; 6/19/1975; R.C. Simpson (LFCC)

- Fort Valley, Powell's Fort east side of Fort; possible old homestead.; 3/20/1977; R.C. Simpson (LFCC)

Ericaceae

Epigaea repens $\mathrm{L}$. (trailing arbutus)

- Camp Roosevelt on the edge of gravel lot in forest. Clay soil.; 5/25/2011; 38.72803,-78.51480; Domangue 258

- Fort Valley off ste. rte. 678, 1/4 mile north of Elizabeth Furnace, 500 yds east (towards buzzard rock) across passage creek, west facing slope, wooded.; 4/13/1980; W.A. Wood 25 (JMUH)

- Great North Mountain, Rt. 768 at Virginia - West Virginia state line.; mixed forest.; Elev. 3000 ft.; 4/18/1978; L. Frey (LFCC)

- Rt. 691, Bear Oak Prescribed Burn, near Va. - W. Va. state line; along roadside of burned area; Flower - white; Elev. 3005 feet.; 4/26/1981; S.M. Collins (LFCC)

Gaultheria procumbens L. (eastern teaberry)

- $\quad$ Rt \#691, Bear Oak Prescribed Burn, near V. - W. Va. state line; Unburned area; Elev. 3005 feet.; 5/21/1981; S. Collins (LFCC)

- $\quad$ Mountains; Woodland; Altitude 800 feet.; 8/18/1966; M. Krouse (LFCC) 
Gaylussacia baccata (Wangenh.) K. Koch (black huckleberry)

- $\quad$ Forest access road, on bank at forest border. Small shrub.; 4/30/2012; 38.96820,-78.52618;

Domangue, M. Lantz 687

- $\quad$ Rt 691, Burn Site; Elev. 3,005 feet.; 7/27/1980; S. Gwyn, S. Collins, J. Hepner (LFCC)

- At Little Ft. Recreation Area in Little Ft. in Massanutten Mt.; 6/19/1975; R.C. Simpson (LFCC)

Kalmia latifolia L. (mountain laurel)

- $\quad$ FR 374 roadside. Shrubby tree $\sim 2$ m tall.; 5/25/2011; 38.72938,-78.56740; Domangue 248

- $\quad$ Rt \#691, Bear Oak Prescribed Burn - near Va. - W. Va. state line; Burned area; Elev. 3005 feet.; 5/24/1981; S. Collins (LFCC)

- Junction Turkey Run, Rt. 55; hillside.; 10/16/1975; Dendrology Class (LFCC)

$\diamond$ Leucothoe racemosa (L.) Gray (swamp doghobble)

Lyonia ligustrina (L.) DC. (maleberry)

- $\quad$ Little Fort Recreation trail.; 6/13/1980; R.C. Simpson (LFCC)

- Little Fort recreation area at Little Fort on the Massanutten Mt.; 6/19/1975; R.C. Simpson (LFCC)

Menziesia pilosa (Michx. ex Lam.) Juss. ex Pers. (minniebush)

- Forest access road, on bank at forest border. Small shrub with glutinous pedicels.; 4/30/2012; 38.96784,-78.52702; Domangue, M. Lantz 686

- $\quad$ Rt \#691, Bear Oak Prescribed Burn, near Va. - W. Va. state line.; Burned Area; Elev. 3005 feet.; 5/22/1981; S. Collins (LFCC)

- Near Peter's Mill bog on trail from Little Ft. to Edinburg Gap.; 6/19/1975; R.C. Simpson (LFCC)

Monotropa hypopithys L. (pinesap)

- Edinburg Gap at the top (on S woods); in deciduous woods, partially covered by leaves; bright red stalk.; 9/4/1975; K.D. Ramsey (LFCC)

- Bear Wallow Trail. George Washington National Forest; woods.; 8/2/1978; S.M. Collins (LFCC)

Monotropa uniflora L. (Indianpipe)

- $\quad$ Growing in woods off Mudhole Bog Trail.; 5/30/2012; 38.91996,-78.37949; Domangue 709

- Mudhole Bog by Little Passage Creek in Little Fort Valley.; Growing in a boggy area near a stream.; 6/28/1972; BIO 68522 (JMUH)

- bog at Little Fort W. woods on trail from Little Fort and Edinburg 2 mi.; Woods at Peter's Mill.; 6/19/1975; R.C. Simpson (LFCC)

- $\quad$ woods near ent. Sign Rt 678 Fort Valley.; 7/25/1976; C.R. Hupp (LFCC)

Rhododendron maximum L. (great laurel)

- Rt. 730 roadside on steep bank at forest border.; 8/23/2011; 38.73852,-78.54751; Domangue 574

- $\quad$ Dry Region, along Rt 620.; 6/3/1975; D.L. McDonald (LFCC)

- Ft. Valley; Massanutten Mountain at Buzzard's Roost on Rt. \#678.; found on slope in a thicket.; 11/1/1976; K.D. Ramsey, W. Bridwell (LFCC) 
Rhododendron periclymenoides (Michx.) Shinners (pink azalea)

- $\quad$ Rt. 730 roadside, at forest border. Small shrub, $1 \mathrm{~m}$ tall.; 4/19/2012; 38.72973,-78.56576;

Domangue 675

- $\quad$ Mt. Jackson; shale mountain.; 5/1983; J. Kauffman (HAVI)

- Liberty Furnace, at junction of rt. 717 and rt. 690.; 9/7/1975; R. Simpson (LFCC)

- $\quad$ Burn site; Elev. 3,005 feet.; 7/7/1980; S. Gwyn, R.C. Simpson (LFCC)

Rhododendron prinophyllum (Small) Millais (early azalea)

- Rt \#691, Bear Oak Prescribed Burn, near Va. - W. Va. state line; in burned area; Pink Rose flowers; Elev. 3005 feet.; 5/22/1981; S. Collins (LFCC)

Rhododendron viscosum (L.) Torr. (swamp azalea)

- $\quad 11 / 2$ mi. E. of Strasburg on the reservoir road.; roadside in deciduous woods; Flowers pinkishwhite; full bloom.; 4/29/1977; R.C. Simpson (LFCC)

- $\quad$ Rt \#691, Bear Oak Prescribed Burn - near Va. - W. Va. state line; unburned area; ; Flower - pink; Elev. 3005 feet.; 5/22/1981; S. Collins (LFCC)

Vaccinium angustifolium Ait. (lowbush blueberry)

- Growing among leaf litter in forest. Small, woody shrub.; 5/11/2011; 38.95301,-78.53149;

Domangue, C.K. McMullen 202

- West of Edinburg GWNF; Little Sluice Trail.; 6/27/1980; R.C. Simpson (LFCC)

- $\quad$ Rt 691, Burn Site; Elev. 3,005 feet.; 8/3/1980; S. Collins, S. Gwyn (LFCC)

Vaccinium pallidum Ait. (Blue Ridge blueberry)

- Growing among leaf litter in forest. Small, woody shrub.; 5/11/2011; 38.95018,-78.53022;

Domangue, C.K. McMullen 203

- $\quad$ Turkey Run, Rt \#55.; Roadside.; 10/16/1975; (LFCC)

- West of Columbia Furnace on Rt. 675 near Wolf Gap. Forestry access Rt. Intersects $675200 \mathrm{ft}$ south in woods.; Rocky area with shallow humus layer acid rock. In association with Quercus prinus, Castanea dentata and Hamamelis virginiana; Elev. 1800 ft.; 10/6/1984; A.D. Wolverton, T. Miller (LFCC)

Vaccinium stamineum L. (deerberry)

- Woodstock, Riverview Park beside Effinger Trail. Low growing shrub.; 5/18/2011; 38.87241, -78.49030; Domangue 225

- Fort Valley, along food trail in woods. Small, woody shrub.; 5/4/2011; 38.88431,-78.37278; Domangue 139

- Massanutten Mountain, Little Fort Recreation Area, along Powell's Mountain Trail (to Edinburg Gap) about 1 mile south of the Little Fort Camping Area; Elev. 1390 ft.; 6/26/1977; R.C. Simpson (LFCC)

- $\quad 1$ 1/2 miles E. of Strasburg on the Reservoir Road; along mountain roadside.; 4/29/1977; R.C. Simpson (LFCC)

Euphorbiaceae

Acalypha gracilens Gray (slender threeseed mercury)

- $\quad$ 1/4 mi. W. Rt. 628; Along cleared path.; Altitude 900 ft.; 9/29/1975; E. Fowlkes (LFCC)

- $\quad$ Mud Hole Gap; Elev. 1250 ft.; 9/19/1974; R.C. Simpson (LFCC) 
Acalypha rhomboidea Raf. (common threeseed mercury)

- Rt. 724 roadside near small stream.; 9/22/2011; 38.78112,-78.77615; Domangue 646

- Public boat landing at N. Fork Shenandoah River, a few meters from Narrow Passage Creek.; 8/2/2011; 38.84594,-78.52970; Domangue 505, 506

- Hockman Homestead 1 mile north of Edinburg. U.S. Rt 11. 100 feet SSE of dwelling; Garden plot.; 10/4/1975; G. Beaty (LFCC)

$\diamond$ Acalypha virginica $\mathrm{L}$. (Virginia threeseed mercury)

Chamaesyce maculata (L.) Small (spotted sandmat)

- Prostrate herb growing in the gravel parking lot of Cedar Grove Church.; 8/2/2011; 38.69426, -78.64740; Domangue 502

Chamaesyce nutans (Lagasca y Segura) Small (eyebane)

- Public boat landing at N. Fork Shenandoah River, a few meters from Narrow Passage Creek.; 8/16/2011; 38.84590,-78.52963; Domangue 556

- Erect herb growing in weedy lot at the intersection of rt. 11 and rt. 620. Rocky soil.; 8/2/2011; 38.69481,-78.64821; Domangue 500

- $\quad$ Approx 5 mi East of Edinburg then S. of \#678 on a shale barren South of road; Elev. 2300.; 7/7/1978; R.C. Simpson (LFCC)

- Approx 5 mile East of Edinburg then S of 678 on a Shale Barren on the mountain South of road; Elev. 2300 ft.; 7/7/1978; R.C. Simpson (LFCC)

$\diamond$ Croton capitatus Michx. var. capitatus (hogwort)*

Euphorbia commutata Engelm. (tinted woodland spurge)

- 1 mi. south of Elizabeth Furnace; Roadside.; 5/2/1978; R. Whiting (LFCC)

- George Washington Nat. Forest, 1 mi. south of Elizabeth Furnace.; 5/1/1978; R.C. Simpson (LFCC)

Euphorbia corollata $\mathrm{L}$. (flowering spurge)

- Rt. 675 (Wolf Gap) roadside at forest border.; 8/8/2011; 38.92067,-78.68912; Domangue 535

- Tomahawk Pond Campground, on steep shale bank near pond.; 7/13/2011; 38.75816,-78.84065; Domangue 438

- $\quad$ FR 274 east.; 7/30/1981; D.E. Miller 71 (JMUH)

- Rt. 675, Wolf's Gap, 1/2 mile east of W. Va. And Va. state line. Campside next to trail.; 7/3/1977; G. Evans (LFCC)

- $\quad$ Prescribed Burn; Elev. 3000 ft.; 9/5/1980; R.C. Simpson (LFCC)

Euphorbia cyparissias L. (cypress spurge) *

- $\quad$ Roadside at rt. 11 and Cedar Ln. intersection.; 4/26/2011; 38.67619,-78.65613; Domangue, M. Lantz 134

- $\quad$ Turn left off Route 11 North onto Route 650. Proced 2.7 miles on left side of road.; along side dirt road.; Elev. 780 Ft.; 5/3/1978; R. Olinger (LFCC)

- Bottom of a shale bank.; Fort Valley, George Washington Nat'l. Park Road \#678, 1/2 mi. S. of "Glass House."; 4/20/1975; R.C. Simpson (LFCC) 
Euphorbia dentata Michx. (toothed spurge) *

- Public boat landing at N. Fork Shenandoah River, a few meters from Narrow Passage Creek.; 8/2/2011; 38.84594,-78.52970; Domangue 509

- Gas station at junction of Rt. \#11 and 81, N. of Strasburg.; 9/5/1975; R.C. Simpson (LFCC)

$\diamond$ Euphorbia lathyris L. (moleplant)*

$\diamond$ Euphorbia spathulata Lam. (warty spurge)

$\diamond$ Phyllanthus caroliniensis Walt. ssp. caroliniensis (Carolina leaf-flower)

Fabaceae

+ Albizia julibrissin Durazz. (silktree) *

- $\quad$ Rt. 672 roadside, a few meters from N. Fork Shenandoah River.; 7/1/2011; 38.84509,-78.53098; Domangue 410

Amphicarpaea bracteata (L.) Fern. (American hogpeanut)

- $\quad$ Rt. 730 roadside at the edge of woods.; 8/23/2011; 38.71286,-78.58624; Domangue 569

Apios americana Medik. (groundnut)

- $\quad$ Little Fort; Muskeg; Altitude 1400.; 7/29/1965; L. Artz (JMUH)

$\diamond$ Astragalus canadensis L. var. canadensis (Canadian milkvetch)

$\diamond$ Astragalus distortus T. \& G. var. distortus (Ozark milkvetch)

$\diamond$ Baptisia australis (L.) R. Br. var. australis (blue wild indigo)

Baptisia tinctoria (L.) R. Br. ex Ait. f. (horseflyweed)

- $\quad$ FR 274 East.; 7/8/1981; D.E. Miller 45 (JMUH)

- Rte. 771 Powell's Fort Camp, Little Fort Valley, Mud Hole Bog.; moist, partly sunny area beside bog.; 7/2/1979; VA Flora Class 3 (JMUH)

- Rt \#691, Bear Oak Prescribed Burn, near Va. - W. Va. state line; along roadbank of burned area; Elev. 3005 feet.; 5/21/1981; S. Collins (LFCC)

- Bog path at Powells Fort.; 7/25/1976; C.R. Hupp (LFCC)

Cercis canadensis $\mathrm{L}$. (eastern redbud)

- Vacant, weedy lot across from Cedar Grove Church on route 620.; 4/18/2011; 38.69491, -78.64812; Domangue, J. Courtwright 86

- $\quad$ Story Trail, off FR 274 East.; 7/8/1981; D.E. Miller 44 (JMUH)

- $\quad$ Roadside.; 9/1/1986; D. Fadely (LFCC)

- Fishers Hill Dam. Fishers Hill, 2 mi south of Strasburg growing on west bank in a deciduous woods.; 4/30/1975; K. Ramsey (LFCC)

Chamaecrista fasciculata (Michx.) Greene (partridge pea)

- Rt. 675 roadside, in weedy area near Stony Creek.; 8/8/2011; 38.86973,-78.63084; Domangue 532

- $\quad$ Rt \#758, 1/2 way up west side of massanutten mts.; 8/9/1975; R.C. Simpson (LFCC) 
Chamaecrista nictitans (L.) Moench (sensitive partridge pea)

- $\quad$ Fort Valley (north end) Rt \#678 across from entrance of Signal Knob trail.; 8/9/1975; R.C. Simpson (LFCC)

Clitoria mariana L. (Atlantic pigeonwings)

- $\quad$ Fort Valley, Rt. 678, Westside; Shale.; 7/13/1975; R. Simpson (LFCC)

- $\quad$ Kings Crossing, massanutten mountains.; 6/27/1975; R.C. Simpson (LFCC)

Coronilla varia L. (crownvetch) *

- Fort Valley, in open, weedy lot. Plant forms a dense, matting groundcover.; 5/25/2011; 38.88446, -78.37760; Domangue 270

- $\quad 1.3$ mi. up from the George Washington National Forest entrance off of Route 678. Roadside environment. Plant is a vine. Wildflower is locally abundant. Inflorescence is white at base to pink at apex and umbel-shaped.; 9/27/2003; M.J. Carmichael 11 (JMUH)

- Chrisman Hollow Road; flower pink and white.; 5/26/1980; S.M. Collins, J.A. Hepner (LFCC)

$\diamond$ Crotalaria sagittalis L. (arrowhead rattlebox)

Desmodium canadense (L.) DC. (showy ticktrefoil)

- $\quad$ Strasburg Park, along fencerow next to open field.; 7/12/2011; 38.97499,-78.35459; Domangue 436

Desmodium canescens (L.) DC. (hoary ticktrefoil)

- .25 mi. W. of Rt. \#628 at Cedar Creek; open Road sides; Elev. 900 ft.; 10/15/1975; E. Fowlkes (LFCC)

Desmodium ciliare (Muhl. ex Willd.) DC. (hairy small-leaf ticktrefoil)

- $\quad$ Edinburg Gap, shale barren, westside; Shale.; 7/13/1975; R.C. Simpson (LFCC)

$\diamond$ Desmodium glabellum (Michx.) DC. (Dillenius' ticktrefoil)

$\diamond$ Desmodium glutinosum (Muhl. ex Willd.) Wood (pointedleaf ticktrefoil)

$\diamond$ Desmodium marilandicum (L.) DC. (smooth small-leaf ticktrefoil)

Desmodium nudiflorum (L.) DC. (nakedflower ticktrefoil)

- $\quad$ FR 274 West.; 7/30/1981; D.E. Miller 69 (JMUH)

$\diamond$ Desmodium nuttallii (Schindl.) Schub. (Nuttall's ticktrefoil)

Desmodium obtusum (Muhl. ex Willd.) DC. (stiff ticktrefoil)

- $\quad$ Rt. 730 roadside at forest border. $1 \mathrm{~m}$ tall.; 7/29/2011; 38.71311,-78.58607; Domangue 477

Desmodium paniculatum (L.) DC. (panicledleaf ticktrefoil)

- Elizabeth Furnace Recreation Area beside dirt trail in woods.; 8/23/2011; 38.92875,-78.32726; Domangue 580 
Desmodium perplexum Schub. (perplexed ticktrefoil)

- $\quad$ Elizabeth Furnace Recreation Area near Passage Creek.; 8/23/2011; 38.92907,-78.32953;

Domangue 583

$\diamond$ Desmodium rotundifolium DC. (prostrate ticktrefoil)

$\diamond$ Desmodium sessilifolium (Torr.) Torr. \& Gray (sessileleaf ticktrefoil)

$\diamond$ Galactia volubilis (L.) Britt. (downy milkpea)

Gleditsia triacanthos L. (honeylocust)

- $\quad$ Large tree beside Cedar Grove Church parking lot.; 5/18/2011; 38.69433,-78.64742; Domangue 213

- Rt 11 in Strasburg near park; Directly along side Rt 11; Elev. 524 ft.; 5/9/1982; W. Crawford, M. Willeford (LFCC)

Kummerowia stipulacea (Maxim.) Makino (Korean clover) *

- $\quad$ Shenandoah Co. Park on the edge of parking lot.; 9/1/2011; 38.93353,-78.45402; Domangue 598

- 1 mile E. of Liberty Furnace, at junction of Rt \#717 and 690; Shale barrens.; 9/7/1975; R.C. Simpson (LFCC)

+ Kummerowia striata (Thunb.) Schindl. (Japanese clover) *

- $\quad$ Powell's Fort Organization Camp (in Massanutten Mountain \} on \#771; Elev. 1500 ft.; 9/12/1978; R.C. Simpson (LFCC)

Lathyrus latifolius L. (perennial pea) *

- $\quad$ Rt. 769 roadside, adjacent open field. Dense population.; 7/29/2011; 38.81577,-78.43133; Domangue 497

- Intersection of rt. 703 and 709. Roadside.; 6/3/2011; 38.81532,-78.66312; Domangue, M. Lantz 300

- Found along a deserted garden approx. 10 miles North of Woodstock on Rt. 707. Plant was growing attached to an old fence row.; 6/30/1977; T. Burke 11 (JMUH)

- 1 mile North of Rt \#55 at wheatfield, along roadside of Rt \#623 west (100 yds.); Along roadside.; 8/13/1978; B. Legge (LFCC)

Lathyrus venosus Muhl. ex Willd. (veiny pea)

- Woodstock Tower.; C. Hinkson (JMUH)

Lespedeza cuneata (Dum.-Cours.) G. Don (sericea lespedeza) *

- Tomahawk Pond Campground, growing in grasses a few meters from pond. Clay soil.; 9/8/2011; 38.75785,-78.84118; Domangue 599

- $\quad$ Rt. 648 along roadside at Sandy Hook, Strasburg.; 10/22/1978; M.L. Snarr (LFCC)

- Rt. 608 at Columbia Furnace along roadside which leads to Wolf Gap.; 10/19/1978; M.L. Snarr (LFCC) 
Lespedeza hirta (L.) Hornem. (hairy lespedeza)

- $\quad$ Roadside, at the intersection of rt. 717 and rt. 611.; 9/8/2011; 38.87644,-78.71243; Domangue 612

- Junction Rt \#717 and 675 west of Columbia Furnace; Shale Barrens; Elev. 900 ft.; 9/7/1975; R.C. Simpson (LFCC)

- Junction of Rt \#717 and Rt. \#675 (west of Columbia Furnace); shale barrens.; 9/7/1975; R.C. Simpson (LFCC)

Lespedeza intermedia (S. Wats.) Britt. (shrubby lespedeza)

- $\quad$ Rt. 730 roadside at the edge of woods near FR 374.; 8/23/2011; 38.72857,-78.56722; Domangue 572

- $\quad$ Prescribed burn; Elev. 3000 ft.; 9/5/1980; R.C. Simpson (LFCC)

$\diamond$ Lespedeza procumbens Michx. (trailing lespedeza)

Lespedeza repens (L.) Bart (creeping lespedeza)

- $\quad$ Rt. 730 roadside at the edge of woods.; 8/23/2011; 38.71288,-78.58598; Domangue 570

- $\quad$ Rt. 678 Fort Valley; shale barrens.; 7/25/1976; C.R. Hupp (LFCC)

- Telephone cut between two woods; Fishers Hill, 2 mi SW of Strasburg.; flowers: pink.; 6/7/1975; K. Ramsey (LFCC)

$\diamond$ Lespedeza virginica $(\mathrm{L}$.) Britt. (slender lespedeza)

$\diamond$ Lotus corniculatus $\mathrm{L}$. (bird's-foot trefoil) *

Lupinus perennis L. (sundial lupine)

- Mt. Olive Burn Site Wolf Gap Road; Dry soils, shale and rocky soil areas.; 5/20/1980; S. Huber (LFCC)

- Rt \#691, Bear Oak Prescribed Burn, near VA - WVA state line; along roadside of burned area; Elev. 3005 ft.; 5/21/1981; S. Collins (LFCC)

Medicago lupulina L. (black medick) *

- $\quad$ Rt. 678 Fort Valley "Glass House."; 7/13/1975; R.C. Simpson (LFCC)

Medicago sativa $\mathrm{L}$. (alfalfa) *

- Just 8.3 miles north of Woodstock town limits. Found in an open pasture field of mixed timothy and alfalfa near a deserted farmstead.; 6/19/1977; T. Burke 10 (JMUH)

Melilotus albus Medik. (white sweetclover)*

- $\quad$ Rt. 749 roadside in grassy clearing.; 6/8/2011; 38.90468,-78.66091; Domangue 311

- $\quad$ Standard Quarry, Strasburg.; 6/17/1975; R.C. Simpson, C. Hupp (LFCC)

- Valley View Orchard west of Strasburg on Route 622; open ground in apple orchard; Elev. 800'.; 8/22/1978; B. Rinker (LFCC) 
Melilotus officinalis (L.) Lam. (sweetclover)*

- $\quad$ Fort Valley, in open, weedy lot. Clay soil.; 5/25/2011; 38.88478,-78.37814; Domangue 268

- $\quad$ Along Rt. 678, off Rt. 675. Along moist roadside bank.; 5/14/1997; Flora of VA Class (JMUH)

- Rte 678, 14.6 miles from King's Crossing (turn left from Rte 675 onto Rte 678); Roadside; Shale barren.; 5/17/1988; Cowherd, Francisco, Thomas 24 (JMUH)

- $\quad 2$ miles east of Strasburg on Rt. 55. 200 feet from Shenandoah River; open field; Elev. 900.; 9/3/1978; M. Hughes (LFCC)

- $\quad$ RT. \#11 at junction with I-81; growing along roadside; Elev. 600 ft.; 5/22/1978; K. Halstead (LFCC)

Robinia pseudoacacia L. (black locust)

- $\quad$ On rt. 623 roadside. Large tree, flowers with heavy fragrance.; 5/11/2011; 38.94017,-78.51963; Domangue, C.K. McMullen 208

- $\quad$ FR 274 West.; 7/22/1981; D.E. Miller 14 (JMUH)

- East of Strasburg on route 678, north end of Massanutten Mt. in Fort Valley at Elizabeth Furnace Campground.; Growing in understory of Oak hickory forest.; Elev. 800'.; 10/3/1977; O. Bate (LFCC)

- On Rt 758 at the bottom of the mountain down the path beside the Leisure Point Sign; tall deciduous trees; Elev. 808 Ft.; 7/4/1979; W. Rush (LFCC)

$\diamond$ Senna hebecarpa (Fernald) Irwin \& Barneby (American senna)

$\diamond$ Senna marilandica (L.) Link (Maryland senna)

Stylosanthes biflora (L.) B.S.P. (sidebeak pencilflower)

- $\quad$ Rt. 678, at "Glass house."; 7/13/1975; R.C. Simpson (LFCC)

- $\quad$ Mine gap, Powells Camp Rd. Fort Valley.; 6/27/1975; R.C. Simpson (LFCC)

Tephrosia virginiana (L.) Pers. (Virginia tephrosia)

- $\quad$ Rte. 678 Fort Valley located on shale barren above roadside.; 7/2/1979; VA Flora Class 13 (JMUH)

- $\quad$ Rt. 678, Fort Valley.; 5/21/1986; BIO 501 (JMUH)

- $\quad$ Fort Valley; Rt. 7582 mi West of Detrick; notes flowers pink.; 5/31/1977; R.C. Simpson (LFCC)

- Peters Mill, Little Fort; Elev. 1450 ft.; 6/20/1975; R.C. Simpson (LFCC)

Trifolium arvense L. (rabbitfoot clover) *

- $\quad$ Fort Valley, growing beside driveway in shale and clay.; 6/24/2011; 38.88448,-78.37763; Domangue, J. Courtwright 388

- Shenandoah Co. Park. Growing in parking lot divider. Clay soil.; 5/30/2011; 38.93381,-78.45457; Domangue 298

- Just outside of Strasburg town limits on Rd. 693.; found along fence row bank that is frequently mowed throughout the summer.; 6/30/1977; T. Burke 4 (JMUH)

- $\quad$ Rt. 678, at "Glass house."; 7/13/1975; R.C. Simpson (LFCC)

- 1 mile north of Rt. \#717 - \#770 junct.; $1 / 2$ mile west of \#770 - \#678 jct.; 7/27/1980;

D. Poffenberger, R. Graves (LFCC)

$\diamond$ Trifolium aureum Pollich (golden clover)* 
Trifolium campestre Schreb. (field clover) *

- $\quad$ Fort Valley, in open, weedy lot. Clay soil.; 5/25/2011; 38.88478,-78.37814; Domangue 267

- Tomahawk Pond Campground, in clearing next to pond. Clay soil.; 5/10/2011; 38.75791, -78.84087; Domangue, B. Burkholder 163

- Hockman Homestead 1 mi. N. of Edinburg on U.S. Rt. 11 E. side of Road; open field grazed by cattle.; 6/13/1980; G. Evans (LFCC)

$\diamond$ Trifolium hybridum L. (alsike clover) *

$\diamond$ Trifolium incarnatum L. (crimson clover)*

Trifolium pratense $\mathrm{L}$. (red clover) *

- $\quad$ Fort Valley, in open, weedy lot. Clay soil.; 5/25/2011; 38.88446,-78.37760; Domangue 275

- $\quad$ Turned left onto Rte 678 at junction of Rte 675 and 678 (King's Crossing). Plant collected 14.6 miles from junction. Roadside.; 5/17/1988; Cowherd, Francisco, Thomas 22 (JMUH)

- $\quad 3.0$ miles NE of Lebanon Church on Rt. 628.; 5/21/1988; Chandler 20 (JMUH)

- $\quad$ Fort Valley Rt 678.; 5/21/1986; (JMUH)

- King's Crossing; Introduced from Europe, red clover has become naturalized throughout almost all of temperate North America.; (JMUH)

- $\quad$ Roadside ditch; Flowers: pink, red.; 5/20/1975; M.T. Bowles (LFCC)

- $\quad$ Rt-601 so., 1 mi so. Stsbg.; Elev. 575 ft.; 9/29/1979; P. Johns (LFCC)

Trifolium repens $\mathrm{L}$. (white clover) *

- Woodstock, Riverview Park. Growing in lawn next to parking lot.; 5/30/2011; 38.87361, -78.49255; Domangue 292

- Woodstock; Turn off Rt. 11 and go 1 1/4 miles East on Lakeview Road and take left on old dirt road and go 3/4 miles; River bottom meadow.; 7/5/1982; J. Nutter 5 (JMUH)

- Hockman Homestead 1 mile north of Edinburg. U.S. Rt 11. $100 \mathrm{ft}$. SSW of dwelling; Garden Plot.; 10/2/1975; G. Beaty (LFCC)

- $\quad$ 1/4 mi. W. of Rt 628 at Cedar Cr.; Open meadow; heart shaped leaflets; Altitude $980 \mathrm{ft}$; 9/28/1975; E. Fowlkes (LFCC)

Trifolium virginicum Small (Kates Mountain clover)

- $\quad$ East of Edinburg, Rt. 675, 3.6 miles East; Shale barren (Appalachian desert-like).; ; C. Hinkson (JMUH)

- $\quad$ Fort Valley; Shale cliffs; Altitude 950.; 5/30/1950; L. Artz (JMUH)

Vicia caroliniana Walt. (Carolina vetch)

- $\quad$ Mooreland Gap Rd., at edge of woods.; 4/26/2011; 38.71054,-78.58953; Domangue, M. Lantz 108

- 3 miles north on Forest Services truck trail from junction with Route 211; Hardwood forest; Flowers are blue; Elev. 1500 feet.; 5/9/1980; L.R. Stewart (LFCC)

- $\quad$ Fort Valley roadside, near picnic area entrance.; 4/1/1977; R.C. Simpson, C. Hupp (LFCC)

+ Vicia sativa $\mathrm{L}$. (garden vetch) *

- Growing on steep bank beside Cedar Creek Rd.; 4/30/2012; 39.02214,-78.46410; Domangue, M. Lantz 690

Vicia villosa Roth ssp. varia (Host) Corb. (winter vetch) *

- $\quad$ Little Fort Valley road; roadside bank.; ; C. Hinkson (JMUH) 
Vicia villosa Roth ssp. villosa (winter vetch)*

- Growing on steep bank beside Cedar Creek Rd.; 4/30/2012; 39.02266,-78.46165; Domangue, M. Lantz 691

Wisteria frutescens (L.) Poir. (American wisteria)

- $\quad$ New Market roadside along fencerow.; 7/1/2011; 38.64759,-78.67840; Domangue 415

Fagaceae

Castanea dentata (Marsh.) Borkh. (American chestnut)

- 2 miles from West Virginia Line and Wolf Gap Rec. area on left side of road; mixed hard woods; Elev. 2400 ft.; 10/8/1978; A. Orndorff (LFCC)

Castanea pumila (L.) Mill. (chinkapin)

- $\quad$ Little Passage Cr.; Dry woods; Altitude 1000 ft.; 8/6/1964; L. Artz (JMUH)

- $\quad$ Fort Valley bog at Powells Fort Camp Little Passage Creek; Elev. 900 ft.; 10/9/1978; L. Copley (LFCC)

- At Little Ft. Recreation Area in Little Ft. in Massanutten Mt.; 6/19/1975; R.C. Simpson (LFCC)

$\diamond$ Fagus grandifolia Ehrh. (American beech)

Quercus alba L. (white oak)

- 1 mile north of Edinburg Rt 11; Eastern Deciduous Forest.; 7/1/1977; R.F. Lytton 8 (JMUH)

- George Washington National Forest, Elizabeth Furnace Picnic Area; field next to stream; Elev. 800 feet.; 9/20/1978; R.E. Keplinger (LFCC)

- 2 miles south of Mt. Olive on Rt 623 on west side of the road; Oak-Hickory forest; Elev. $1000 \mathrm{ft}$.; 10/15/1978; C. Whittington (LFCC)

Quercus bicolor Willd. (swamp white oak)

- $\quad$ Elizabeth Furnace; Wet soil; Altitude 800.; 11/4/1964; L. Artz (JMUH)

Quercus coccinea Muenchh. (scarlet oak)

- Woodstock, Riverview Park, in forest. Large tree.; 8/16/2011; 38.87316,-78.48961; Domangue 560

Quercus ilicifolia Wangenh. (bear oak)

- $\quad$ Story Trail, off FR 274 (overlook).; 7/30/1981; D.E. Miller 68 (JMUH)

- $\quad$ Rt 691, Burn site; Elev. 3,005 feet.; 8/3/1980; S. Gwyn, S. Collins (LFCC)

- East of Strasburg on route 678, north end of Massanutten Mt. in Fort Valley at Elizabeth Furnace Campground.; Growing in understory of oak hickory forest.; Elev. 800'.; 10/3/1977; O. Bate (LFCC)

Quercus imbricaria Michx. (shingle oak)

- $\quad$ Fort Valley; Shale soil. Dry; Altitude 1200 ft.; 9/12/1963; L. Artz (JMUH)

- South Jackson on Railroad Street behind D.S. Hottinger's House; woods that has been burnt several times with very rich soil; Elev. 1546.; 11/6/1985; D. Hottinger, D. Rosenberger (LFCC)

- Rt 678 1/2 mile north of Seven Fountains Post Office; Fort Valley.; 6/16/1978; R.C. Simpson (LFCC) 
+ Quercus macrocarpa Michx. (bur oak)

- $\quad 2$ mile west of JCT R \#525 and Rt. 55 on Rt. 55 at North Fork Campground along Shenandoah

River; River Bank; note tripartate hairs.; 6/18/1978; J. Brumback (LFCC)

- Rt. 767, go off at B.W. Price house and up the lane to intersection at main road and house; drive $200 \mathrm{ft}$. from road next to deer trail (3 mi N. Quicksburg).; 2/12/1980; S. Hollsinger (LFCC)

Quercus marilandica Muenchh. (blackjack oak)

- $\quad$ Devil's Backbone State Forest, beside dirt road. Large tree.; 7/20/2011; 39.04715,-78.43126;

Domangue, C.K. McMullen 464

- Woodstock; George Washington National Forest; Woodstock Tower.; 10/3/1979; K. Motiska, K. Halstead (LFCC)

- $\quad$ Fort Valley, on Little Fort Trail 1 mile from its junction with Rt. 730.; 7/17/1975; R. \& H. Simpson (LFCC)

Quercus montana L. (chestnut oak)

- 3 miles from West Virginia Line and Wolf Gap Rec. area on left side of road; mixed hard woods; Elev. 2200 ft.; 10/8/1978; A. Orndorff (LFCC)

- $\quad$ Go Rt 42-S for 5.7 miles until it intersects with Rt 675-W. Go 675-W for 7 miles until you reach Wolf Gap recreational area. Specimens collected along Big Blue hiking trail.; Oak-Hickory forest. Dry mesic conditions.; Elev. 2800 ft.; 10/8/1978; L. Leta (LFCC)

Quercus muehlenbergii Engelm. (chinkapin oak)

- 1 mile north of Edinburg on Rt. 11; Eastern Deciduous Forest; Appears to be hybridizing with other oaks.; 7/1/1977; R.F. Lytton 11 (JMUH)

Quercus palustris Muenchh. (pin oak)

- Peter's Mill Run; Moist soil - swamp; Altitude 1200 ft.; 8/6/1964; L. Artz (JMUH)

- Buckhorn state resort; deciduous and confer trees.; 9/16/1979; W. Rush (LFCC)

$\diamond$ Quercus prinoides Willd. (dwarf chinkapin oak)

Quercus rubra L. (northern red oak)

- $\quad$ East of Edinburg.; Dry woods.; 5/18/1983; BIO 50112 (JMUH)

- 100 yds. S of basketball ct. at Ft. Valley Bog; Altitude 1300 feet.; 7/18/1971; C.A. Helsley (JMUH)

- $\quad$ Go Rt 42-S for 5.7 miles until it intersects with Rt. 675-W. Go 675-W until you come to Wolf Gap recreation area. Specimens collected along the Big Blue hiking trail.; Oak-Hickory Forest. Dry mesic conditions.; Elev. 2800.; 10/8/1978; L. Leta (LFCC)

- Woodstock Tower; George Washington Nat. Forest.; Oak forest.; 10/7/1979; J. Evans (LFCC)

Quercus shumardii Buckley (Shumard's oak)

- Woodstock, Riverview Park, in forest. Large tree.; 8/16/2011; 38.87316,-78.48961; Domangue 559 
Quercus stellata Wangenh. (post oak)

- Dry sandy or gravelly soil; Rt. 678, Fort Valley.; 5/21/1986; (JMUH)

- Found 20 miles E. of Edinburg on shale barren on roadside of Rt. 678.; 6/6/1905; R. Berg, S. Chappell 107 (JMUH)

- 20.6 miles east of Edinburg on State Rte. 678.; On dry, poor soils.; 5/16/1984; S. Mason (JMUH)

- South Jackson behind D.S. Hottinger's house on Railroad Street; Small woods burnt often; Elev. 1546.; 11/6/1985; D. Hottinger, D. Rosenberger (LFCC)

- At Columbia Furnace beside a church and graveyard; associated with Q. alba and Q. rubra.; 9/7/1975; R.C. Simpson (LFCC)

Quercus velutina Lam. (black oak)

- $\quad$ Woodstock Tower.; 11/9/1980; K. Simpson (LFCC)

\section{Fumariaceae}

Corydalis flavula (Raf.) DC. (yellow fumewort)

- Woodstock, Riverview Park. On bank along Effinger Trail. Growing among leaf litter.; 4/5/2011; 38.87252,-78.49018; Domangue 77

- Along hwy 754 approx. 200 yds. north of where the road crosses North Fork of Shenandoah River.; on south-facing flood plain slope in woods.; 4/15/1978; M. Kelly (JMUH)

Corydalis flavula (Raf.) DC. (yellow fumewort)

- On route 675, 10.1 miles from junction of route 11 and Edinburg.; hillside on side of road in rocky soil.; 4/6/1982; J. Beer (JMUH)

$\diamond$ Corydalis sempervirens (Raf.) DC (rock harlequin)

$\diamond$ Dicentra canadensis (Goldie) Walp. (squirrel corn)

Dicentra cucullaria (L.) Bernh. (dutchman's breeches)

- Strasburg Park, growing beside trail a few meters from N. Fork Shenandoah River. Sandy soil.; 3/22/2012; 38.97360,-78.35233; Domangue 664

- $\quad$ Fort Valley, beside Ramsey Rd. on steep bank above creek.; 4/26/2011; 38.89257,-78.37066; Domangue, M. Lantz 128

- Along north side of hwy 648, 1/4 mile east of junction 648 and 11.; wooded north-facing slope.; 4/15/1978; M. Kelly (JMUH)

+ Dicentra eximia (Ker-Gawl.) Torr. (turkey corn)

- $\quad$ On Rt. 55, approx. 7 miles west of interstate 81. Located on right side of Rt. 55.; Elevation approx. $500 \mathrm{~m}$ on flat portion of mountain ridge. Soil medium in texture. Located in a clearing of wooded deciduous forest.; 4/15/2006; T. Dean 6 (JMUH)

\section{Gentianaceae}

$\diamond$ Bartonia virginica (L.) B.S.P. (yellow screwstem)

$\diamond$ Gentiana clausa Raf. (bottle gentian)

$\diamond$ Gentiana saponaria L. (harvestbells) 
$\diamond$ Gentiana villosa L. (striped gentian)

Sabatia angularis (L.) Pursh (rosepink)

- $\quad$ Fort Valley; 5 miles north of Elizabeth Furnace; Elev. 800 ft.; 8/4/1980; R.C. Simpson (LFCC)

- 1 mi. north of Kings Crossing (store) on east road side bank.; Flowrs: pink.; 7/28/1975; R. \& H. Simpson (LFCC)

\section{Geraniaceae}

Erodium cicutarium (L.) L'Hér. ex Ait. (redstem stork's bill) *

- Mt. Jackson Park, western end of Wunder St., on slope next to tennis courts (mowed lawn); 3/21/2011; 38.75048,-78.64680; Domangue, J. Courtwright 65

Geranium carolinianum L. (Carolina geranium)

- $\quad$ Woodstock, Riverview Park. Growing in lawn beside gazebo.; 5/18/2011; 38.87339,-78.49358; Domangue 220

- Grassy portion between two woods, a power line cut. Fishers Hill, 2 mi S.W. of Strasburg.; Flowers: purple.; 6/7/1975; K. Ramsey (LFCC)

$\diamond$ Geranium dissectum L. (cutleaf geranium) *

Geranium maculatum L. (spotted geranium)

- $\quad$ Mooreland Gap Rd., at edge of woods.; 4/26/2011; 38.71054,-78.58953; Domangue, M. Lantz 109

- Near entrance to Powell's trail Route 675, Massanutten Mountain; Woods.; 5/16/1982; L. Michael Hill 1 (BDWR)

- Rt. 628; roadside; 5 mi. south of Va Rt. 55.; 5/3/1975; M. Dyson-Cobb (LFCC)

- Rt. \#678 1 mile south of Elizabeth Furnace; Growing on side of road; Elev. 725 ft.; 5/22/1978;

K. Halstead (LFCC)

Geranium molle L. (dovefoot geranium) *

- $\quad$ Elizabeth Furnace Recreation Area, growing in parking lot divider.; 4/19/2012; 38.92766, -78.32954; Domangue 681

- Mt. Jackson; shale mountain.; 5/1983; J. Kauffman (HAVI)

- $\quad$ Approx. 2 miles North of Woodstock off US RT. 11 at Pugh's Run; Limestone outcrop near water; fls. Lavender - geranium-like.; 6/5/1977; R.C. Simpson, K.D. Ramsey (LFCC)

- $\quad$ Rt. 658, 1.3 mi N Rt 675 NW of Edinburg; Elev. 850 ft.; 6/19/1977; G. Evans, K. Ramsey, R.C. Simpson (LFCC)

\section{Grossulariaceae}

$\diamond$ Ribes americanum Mill. (American black currant)

Ribes rotundifolium Michx. (Appalachian gooseberry)

- Woodstock Reservoir.; 5/27/1980; R.C. Simpson (LFCC) 
Haloragaceae

Proserpinaca palustris L. (marsh mermaidweed)

- $\quad$ Rt 685, 1.3 mi N. Rt 675 (NW Edinburg); Elev. 850 ft.; 6/19/1977; G. Evans, K. Ramsey, R.C. Simpson (LFCC)

Hamamelidaceae

Hamamelis virginiana $\mathrm{L}$. (American witchhazel)

- $\quad$ Elizabeth Furnace Recreation Area on forest trail. Medium-sized tree.; 8/23/2011; 38.92589, -78.32782; Domangue 578

- $\quad$ Rte. 771 Powell's Fort Camp, Little Fort Valley, Mud Hole Bog.; shady moist area in bog.; 7/2/1979; VA Flora Class 4 (JMUH)

- $\quad$ Power line 300 yds. E. of Rt 717; 4 mi. NE of Basye; moist, near wet weather creek.; 5/17/1984; S. Arey 14 (JMUH)

- Northern Massanutten Mountains; Mudhole Bog.; 7/28/1978; B. Latham 47 (JMUH)

- $\quad$ Powells Fort Camp; Bog.; 10/8/1979; T. Leight, C. Hupp, R.C. Simpson (LFCC)

- $\quad$ Middle Mt.; 8/8/1965; M. Krouse (LFCC)

Hippocastanaceae

+ Aesculus glabra Willd. (Ohio buckeye)

- Growing in lawn beside Flat Rock Church. Large tree. Many saplings nearby.; 5/9/2012; 38.70015,-78.74574; Domangue 694

Hydrangeaceae

Hydrangea arborescens $\mathrm{L}$. (wild hydrangea)

- $\quad$ FR 274 East.; 7/22/1981; D.E. Miller 18 (JMUH)

- $\quad$ Rt. 678 up from Kings Crossing.; 5/21/1986; BIO 501 (JMUH)

- Fort Valley at Peter's Mill Run (Little Fort).; 6/20/1975; R.C. Simpson (LFCC)

- Woodstock; Woodstock Tower George Washington national Forest.; 10/14/1979; J. Evans (LFCC)

$\diamond$ Philadelphus coronarius L. (sweet mock orange) *

$$
\text { Hydrophyllaceae }
$$

$\diamond$ Hydrophyllum virginianum $\mathrm{L}$. (eastern waterleaf)

Phacelia dubia (L.) Trel. (smallflower phacelia)

- $\quad$ Fort Valley area.; 5/18/1984; BIO 501138 (JMUH) 
Juglandaceae

Carya alba (L.) Nutt. ex Ell. (mockernut hickory)

- East of Strasburg on Route 678, north end of Massanutten Mt. in Fort Valley at Elizabeth Furnace Campground.; Growing in understory of oak hickory forest.; Elev. 800'.; 10/3/1977; O. Bate (LFCC)

- 2 miles south of Mt. Olive on Rt. 623 on west side of road in flat woods; Oak-Hickory Forest; Elev. 1000 ft.; 10/15/1978; C. Whittington (LFCC)

Carya cordiformis (Wangenh.) K. Koch (bitternut hickory)

- $\quad$ Little Passage Cr.; Moist woods; Altitude 1000 ft.; 8/6/1964; L. Artz (JMUH)

Carya glabra (Mill.) Sweet (pignut hickory)

- $\quad$ Splinters Ln. roadside off rt. 826.; 9/15/2011; 38.69604,-78.61985; Domangue 622

- $\quad$ Rt 675 East, 2 miles east of Edinburg; Shale Barren; stunted.; 6/22/1977; R.F. Lytton 4 (JMUH)

- $\quad 1.3$ mi. up from the George Washington National Forest entrance off of Route 678. Trailside environment. Surrounding forest was predominantly pine, rhododendron, and sassafras. Tree was 15 ft. tall. Leaflets pubescent, 5 per. Leaf. Bark gray and slightly furrowed.; 9/27/2003; M.J. Carmichael 5 (JMUH)

Carya ovalis (Wangenh.) Sarg. (red hickory)

- 2 miles south of Mt. Olive on Rt. 623 on west side of road in Flat woods; Oak-Hickory forest; Elev. 1000 ft.; 10/15/1978; C. Whittington (LFCC)

Carya ovata (Mill.) K. Koch (shagbark hickory)

- Growing along the fence line of a pasture once used for livestock. Large tree.; 5/4/2011; 38.87190,-78.57690; Domangue 155

- $\quad$ Harmony Hall (Strasburg, VA); mixed hardwood along Centry Lane.; 9/9/1976; M.F. Heurich (LFCC)

$\diamond$ Juglans cinerea $\mathrm{L}$. (butternut)

Juglans nigra L. (black walnut)

- 1 mi. So. Strasburg, 3/4 mi. S. East intersection of Rt 11 on 601; Elev. 575 ft.; 8/7/1979; P. Johns (LFCC)

\section{Lamiaceae}

$\diamond$ Agastache nepetoides (L.) Kuntze (yellow giant hyssop)

$\diamond$ Blephilia ciliata (L.) Benth. (downy pagoda-plant)

Clinopodium calamintha (L.) Stace (lesser calamint) *

- Shenandoah Co. Park, beside grassy path in lightly wooded area. Fragrant.; 8/16/2011; 38.93762, -78.45095; Domangue 563 
Clinopodium vulgare $\mathrm{L}$. (wild basil)

- $\quad$ Elizabeth Furnace Recreation Area beside parking lot.; 6/24/2011; 38.92763,-78.32986;

Domangue, J. Courtwright 379

- Woodstock; Turn off Rt. 11 and go 1 1/4 miles East on Lakeview Road and take left on old dirt road and go 3/4 miles; River bottom meadow.; 7/5/1982; J. Nutter 12 (JMUH)

- $\quad$ 1/4 mi w. of Rt. 628 at Cedar Cr.; cleared path; red (pink) bloom; Altitude 900 ft.; 9/29/1975; E. Fowlkes (LFCC)

Collinsonia canadensis L. (richweed)

- $\quad$ Little Fort V.; Stream Bank; Altitude 1400.; 7/28/1965; L. Artz (JMUH)

- 1 mi. N. of Liberty Furnace on 691.; flood plain of Laurel Run.; 9/7/1975; R.C. Simpson (LFCC)

Cunila origanoides (L.) Britt. (common dittany)

- $\quad$ Roadside, at the intersection of rt. 717 and rt. 611. Minty scent.; 9/8/2011; 38.87644,-78.71243; Domangue 613

- $\quad$ Rt. 657, 3.7 mi. east of Edinburg.; Shale Barren.; 5/18/1983; J. Poudrier, S. Smith 5 (JMUH)

- Junction of Rt. \#717 and 675 west of Columbia Furnace; Shale Barren; Elev. 900 ft.; 9/7/1975; R.C. Simpson (LFCC)

- $\quad$ Edinburg Gap, shale barren, westside; shale.; 7/13/1975; R.C. Simpson (LFCC)

Glechoma hederacea L. (ground ivy) *

- Bear Oak Prescribed Burn, Rt \#691, near Va. - W. Va. state line; on roadbank of unburned side; Flower - purple/blue; Elev. 3005 feet.; 5/16/1981; S.M. Collins (LFCC)

Glechoma hederacea L. (ground ivy) *

- Shenandoah Co. Park. Low growing, creeping plant in lawn.; 5/18/2011; 38.93691,-78.45180; Domangue 234

- $\quad$ Grassy bank along Bank St. in Mt. Jackson.; 3/18/1990; J.E. Irre 15 (JMUH)

- Hockman Homestead ("Chequers") 1 mile north of Edinburg on U.S. Route 11 on east side of road; Area grazed by cattle, grasses, daisies, cedars.; 6/13/1977; G. Evans, J. Evans (LFCC)

Hedeoma pulegioides (L.) Pers. (American false pennyroyal)

- Growing in gravel lot in forest off rt. 691. Strong scent.; 9/8/2011; 38.89268,-78.70621; Domangue 616

Lamium amplexicaule $\mathrm{L}$. (henbit deadnettle) *

- Woodstock, Riverview Park. On edge of parking lot.; 3/29/2011; 38.87452,-78.49072; Domangue 72

- $\quad$ East side of Rt 42, southern point of Conicville, VA township limit.; Disturbed agricultural field.; 4/13/1985; M.L. Verts 340 (JMUH)

- New Market, .25 mi south of junction Rt \#11 and Rt \#737 on Rt \#11; open field along roadside; Elev. 1000 ft.; 5/2/1978; R. Whiting, L. Frey, J. Brumback (LFCC)

- $\quad$ Near Strasburg, Junct. Rt 11 and I-81 on Frontage Road; mustard field; Elev. 640 ft.; 4/17/1977; (LFCC) 
Lamium purpureum L. (purple deadnettle) *

- Shenandoah Co. Park. In lawn directly adjacent to tennis courts.; 4/5/2011; 38.93515,-78.45244; Domangue 81

- 1 mile north of Woodstock on west side of U.S. 11; Roadside lawn.; 4/24/1982; E. Leavy 31 (JMUH)

- Gospel St. in Mt. Jackson, across from 5875 next to mailbox.; 3/11/1990; J.E. Irre 7 (JMUH)

Leonurus cardiaca $\mathrm{L}$. (common motherwort) *

- $\quad$ Roadside, at rt. 11 and rt. 672 intersection.; 6/15/2011; 38.84656,-78.53180; Domangue 341

- Rte. 678 Fort Valley along roadside, sunny field.; 7/2/1979; VA Flora Class 12 (JMUH)

Lycopus americanus Muhl. ex W. Bart. (American water horehound)

- $\quad$ Rt. 730 roadside at the edge of woods.; 8/23/2011; 38.70948,-78.62437; Domangue 567

- $\quad$ Fort Valley, Rt 678 near Eliz. Furn. Picnic.; field.; 7/25/1976; C.R. Hupp (LFCC)

Lycopus virginicus L. (Virginia water horehound)

- 1 mi. N. of Liberty Furnace on Rt. \#691.; Laurel run flood plain.; 9/7/1975; R.C. Simpson (LFCC)

- $\quad$ G. W. Nat. Forest, Little Fort, Peter's Mill Run.; 9/11/1980; R.C. Simpson, M. Willeford (LFCC)

$\diamond$ Marrubium vulgare L. (horehound) *

Mentha arvensis $\mathrm{L}$. (wild mint)

- $\quad$ Rt. 730 roadside at the edge of woods.; 8/23/2011; 38.70948,-78.62437; Domangue 566

Mentha spicata $\mathrm{L}$. (spearmint) *

- $\quad$ Route 764 Below second house; Light purple flowers.; Roadside.; 7/2/1987; Jennifer Estep (BDWR)

- $\quad$ Found Rt. 6032 mi. from Woodstock; Roadside, wasteland.; 7/6/1977; B. Kennedy (JMUH)

Mentha x piperita L. (peppermint) *

- $\quad$ Rt. 717 roadside (Jerome) below shale barrens. Minty scent.; 9/8/2011; 38.85925,-78.72931; Domangue 609

+ Mentha $x$ rotundifolia $($ L.) Huds. *

- $\quad$ Rt. 717 roadside half a km from Orkney Grade. Shale/clay soil. Plant with minty scent.; 9/8/2011; 38.81030,-78.79507; Domangue 604

Monarda clinopodia L. (white bergamot)

- Elizabeth Furnace Recreation Area, in riparian zone near Passage Creek.; 6/24/2011; 38.92887, -78.33064; Domangue, J. Courtwright 375

Monarda fistulosa L. (wild bergamot)

- Fort Valley, on the edge of a pond in open pasture.; 6/24/2011; 38.88404,-78.37979; Domangue, J. Courtwright 384

- $\quad$ FR 274 West.; 7/8/1981; D.E. Miller 40 (JMUH) 
Nepeta cataria L. (catnip) *

- Rt. 730 roadside in forest clearing. Minty scent.; 6/24/2011; 38.71222,-78.58757; Domangue, J. Courtwright 357

- $\quad$ Route 764 Below second house; White flowers.; Roadside.; 7/3/1987; Jennifer Estep (BDWR)

- $\quad$ FR 274 West.; 7/8/1981; D.E. Miller 33 (JMUH)

- Specimen found Rt. 603, 2 mi. from Woodstock; Roadside, wasteland.; 7/6/1977; B. Kennedy (JMUH)

- $\quad$ Edinburg; open field; Elev. 800 ft.; 8/25/1978; B. Baker (LFCC)

Perilla frutescens (L.) Britt. (beefsteakplant) *

- Growing in gravel lot in forest off rt. 691. Strong scent.; 9/8/2011; 38.89268,-78.70621; Domangue 614

Prunella vulgaris $\mathrm{L}$. (common selfheal)

- In cow pasture of weeds and woody plants.; 6/30/2011; 38.79396,-78.65941; Domangue 397

- $\quad$ FR 274 East.; 7/8/1981; D.E. Miller 31 (JMUH)

- $\quad$ Northern Massanutten Mountains; Mudhole Bog.; 7/28/1978; B. Latham 37 (JMUH)

- 2 miles east of Strasburg on Rt. \#55. 50 feet from Shenandoah River; open field; Elev. 850 ft.; 10/23/1978; M. Hughes (LFCC)

- $\quad$ Fort Valley, Rt. 678 streambank at W ent. To Geo. Wash. Natl; streambank.; 7/25/1976; C.R. Hupp (LFCC)

Pycnanthemum incanum Muhl. (hoary mountainmint)

- $\quad$ Red Robin Ln. roadside, on grassy bank.; 7/20/2011; 38.94599,-78.52652; Domangue, C.K. McMullen 474

- $\quad$ Big Springs Fort Valley; Springs.; ; R. Simpson (LFCC)

- $\quad$ Fort Valley; 5 miles north of Elizabeth Furnace; Elev. 800 ft.; 8/4/1980; R.C. Simpson (LFCC)

+ Pycnanthemum pycnanthemoides (Leavenworth) Fern. (southern mountainmint)

- $\quad$ FR 274 West.; 7/22/1981; D.E. Miller 28 (JMUH)

Pycnanthemum tenuifolium Schrad. (narrowleaf mountainmint)

- Devil's Backbone State Forest, beside dirt road.; 7/20/2011; 39.04836,-78.43542; Domangue, C.K. McMullen 472

- Elizabeth Furnace Recreation Area, beside grassy field at forest border.; 6/24/2011; 38.92759, -78.32863; Domangue, J. Courtwright 371

- $\quad$ Little Fort; Border of Bog; Altitude 1400; 7/29/1965; L. Artz (JMUH)

- $\quad$ Rt. 648 along roadside at Sandy Hook, Strasburg.; 10/22/1978; M.L. Snarr (LFCC)

Pycnanthemum verticillatum (Michx.) Pers. (whorled mountainmint)

- $\quad$ Fort Valley, Big Spring.; 7/17/1975; R. \& H. Simpson (LFCC)

Salvia lyrata L. (lyreleaf sage)

- Mine Mountain Rd., on roadside at forest border.; 5/25/2011; 38.89643,-78.40716; Domangue 265

- Roadside ditch.; 5/20/1975; M.T. Bowles (LFCC)

- North of Tom's brook on \#651 about .6 mi. west of \#11 between \#11 and \#81; old overgrown woodland trail.; 5/4/1977; R.C. Simpson (LFCC)

$\diamond$ Salvia urticifolia $\mathrm{L}$. (nettleleaf sage) 
$\diamond$ Salvia verbenacea $\mathrm{L}$. (wild clary)*

Scutellaria elliptica Muhl. ex Spreng var. elliptica (hairy skullcap)

- $\quad$ Shenandoah Co. Park, in weedy clearing in woods. Clay soil.; 6/15/2011; 38.93513,-78.45619; Domangue 348

- Devil's Backbone State Forest beside stream.; 6/8/2011; 39.04911,-78.43460; Domangue 321

Scutellaria elliptica Muhl. ex Spreng (hairy skullcap)

- 3 mi. NE of Strasburg at the Strasburg Reservoir.; by a stream.; 6/17/1975; R.C. Simpson, C. Hupp (LFCC)

- $\quad$ Peter Mill; Little Fort.; 6/20/1975; R.C. Simpson (LFCC)

Scutellaria integrifolia $\mathrm{L}$. (helmet flower)

- Elizabeth Furnace Recreation Area, beside grassy field at forest border.; 6/24/2011; 38.92759, -78.32863; Domangue, J. Courtwright 372

- $\quad$ Little Fort V.; Bog; Altitude 1400.; 7/8/1965; L. Artz (JMUH)

- Massanutten Mountain .6 mi. East of the Little fort recreation area; moist area along roadside.; 6/26/1977; R.C. Simpson (LFCC)

Scutellaria lateriflora $\mathrm{L}$. (blue skullcap)

- Duncan Hollow; Dry woods; Altitude 1250.; 7/21/1965; L. Artz (JMUH)

Scutellaria ovata Hill (heartleaf skullcap)

- $\quad$ Fort Valley; Shale; Altitude 1200.; 7/9/1963; L. Artz (JMUH)

$\diamond$ Scutellaria parvula Michx. (small skullcap)

Scutellaria serrata Andr. (showy skullcap)

- $\quad$ Fort Valley; Rich woods.; 5/16/1964; L. Artz (JMUH)

- One mile south of George Washington National Forest Sign on 616 and left hand side of road; In damp ground in deciduous woods; Elev. 600.; 6/1/1978; H.E. Reed (LFCC)

- Bog stream, Little Fort 1 mi. s. of rec. area.; 5/22/1976; C.R. Hupp (LFCC)

$\diamond$ Stachys germanica L. (German hedgenettle)*

$\diamond$ Stachys hispida Pursh (smooth hedgenettle)

Teucrium canadense L. (Canada germander)

- Growing in weedy lot at the intersection of rt. 11 and rt. 620.; 7/12/2011; 38.69518,-78.64851; Domangue 418

- In cow pasture next to stream.; 6/30/2011; 38.79452,-78.65999; Domangue 404

- $\quad$ Fort Valley; along side of road, fls white (albino) very fragrant.; 7/5/1981; M. Krouse (LFCC)

- $\quad$ Bog woods Powells.; 7/25/1976; C.R. Hupp (LFCC)

$\diamond$ Trichostema brachiatum L. (fluxweed)

Trichostema dichotomum $\mathrm{L}$. (forked bluecurls)

- $\quad$ Tomahawk Pond Campground on the edge of pond. Clay soil.; 9/8/2011; 38.75895,-78.84062; Domangue 601 
$\diamond$ Trichostema setaceum Houtt. (narrowleaf bluecurls)

\section{Lauraceae}

Lindera benzoin (L.) Blume (northern spicebush)

- $\quad$ Tomahawk Pond Campground, in lightly forested area near small stream. Shrubby tree.; 6/8/2011; 38.75983,-78.84111; Domangue 306

- Massanutten Mountains (northern); Mudhole Bog.; 7/28/1978; B. Latham 48 (JMUH)

- Massanutten Mountains; Moist woods; Altitude 800-900 ft.; 8/7/1966; M. Krouse (LFCC)

- 1 mile Southwest on Forest Service Road \#274 at Lion's Tale Trail parking lot; Elev. 1300 ft.; 9/27/1982; J. Lonas, T. McCracken (LFCC)

Sassafras albidum (Nutt.) Nees (sassafras)

- $\quad$ Growing beside foot trail in woods near the intersection of rt. 730 and FR 374.; 7/29/2011; 38.72910,-78.56693; Domangue 484

- 1 mile north of Edinburg on Rt. 11; Open meadow.; 7/1/1977; R.F. Lytton 9 (JMUH)

- $\quad$ Story Trail, off FR 274.; 7/8/1981; D.E. Miller 36 (JMUH)

- $\quad$ Rt. 691, Barn Site; Road side; Elev. 3,005 feet.; 7/27/1980; S. Collins, S. Gwyn, J. Hepner (LFCC)

- 2 miles from West Virginia Line and Wolf Gap Rec. area on left side road; mixed hard woods; Elev. 2400 ft.; 10/8/1978; A. Orndorff (LFCC)

Lentibulariaceae

$\diamond$ Utricularia gibba L. (humped bladderwort)

Linaceae

$\diamond$ Linum medium (Planch.) Britton var. texanum (Planch.) Fern. (stiff yellow flax)

Linum striatum Walt. (ridged yellow flax)

- Devil's Backbone State Forest, in grassy clearing in woods.; 7/20/2011; 39.04879,-78.43174; Domangue, C.K. McMullen 467

$\diamond$ Linum sulcatum Riddell var. sulcatum (grooved flax)

$\diamond$ Linum virginianum $\mathrm{L}$. (woodland flax)

Lythraceae

Cuphea viscosissima Jacq. (blue waxweed)

- $\quad$ Rt. 717 roadside below steep shale bank. Plant glutinous.; 8/8/2011; 38.85930,-78.72937; Domangue 539

- $\quad$ Edinburg Gap, shale barren, westside; Shale.; 7/13/1975; R.C. Simpson (LFCC)

- $\quad$ 1/4 mi, W of Rt. 628 at Cedar Cr.; Creek bank; purple bloom hairy stems; Altitude $800 \mathrm{ft}$; 9/29/1975; E. Fowlkes (LFCC) 
Magnoliaceae

Liriodendron tulipifera L. (tuliptree)

- $\quad$ Elizabeth Furnace Recreation Area. Large tree near parking lot.; 7/29/2011; 38.92763,-78.32915;

Domangue 489

- East of Strasburg on route 678, north end of Massanutten Mt. in Fort Valley at Elizabeth Furnace Campground.; Growing in understory of Oak hickory forest.; Elev. 800'.; 10/3/1977; O. Bate (LFCC)

- 2 miles from West Virginia Line and Wolf Gap Rec. area on left side road; mixed hard woods; Elev. 2400 ft.; 10/8/1978; A. Orndorff (LFCC)

Magnolia acuminata (L.) L. (cucumber-tree)

- $\quad$ Little Passage Cr.; Woods.; 8/6/1964; L. Artz (JMUH)

- $\quad$ Off State Rt \#720, Barb's Gap; along stream.; 9/20/1980; J.A. Hepner, S.M. Collins (LFCC)

$\diamond$ Magnolia tripetala (L.) L. (umbrella-tree)

Malvaceae

Abutilon theophrasti Medik. (velvetleaf) *

- Rt. 720 roadside near Meems Bottom Covered Bridge, growing beside cultivated field.; 9/15/2011; 38.72056,-78.65414; Domangue 623

$\diamond$ Hibiscus moscheutos L. var. moscheutos (crimsoneyed rosemallow)

+ Hibiscus syriacus $\mathrm{L}$. (rose of Sharon) *

- Growing beside gravel driveway next to open field. Small shrub.; 7/20/2011; 38.94162,-78.51902; Domangue, C.K. McMullen 476

Hibiscus trionum L. (flower of an hour) *

- Hockman Homestead 1 mile north of Edinburg. U.S. Rt 11; $100 \mathrm{ft}$. SSW of dwelling; Garden plot.; petals: yellow.; 10/2/1975; G. Beaty (LFCC)

Malva neglecta Wallr. (common mallow) *

- $\quad$ Fort Valley, in open, weedy lot on dirt pile.; 5/25/2011; 38.88478,-78.37814; Domangue 269

- $\quad 3.0$ miles NE of Lebanon Church on Rt. 628.; 5/21/1988; Chandler 3 (JMUH)

- $\quad 3.0 \mathrm{mi}$. NE of Lebanon Church on Route 628. Floodplain of stream.; Edible parts: Young leaves eaten raw or added to soups and stews. Cheeselike fruits eaten raw or the seeds extracted and ground into flour.; 5/21/1988; Cason, Gordon 24 (JMUH)

Malva sylvestris $\mathrm{L}$. (high mallow) *

- Fishers Hill on Rt \#601 at the "old mill"; grown over yard; Flowers: purple.; 7/2/1975; K.D. Ramsey (LFCC)

$\diamond$ Sida spinosa $\mathrm{L}$. (prickly fanpetals) * 


\section{Menispermaceae}

Menispermum canadense L. (common moonseed)

- $\quad$ Pugh's Run; Limestone cliffs; Altitude 1250.; 6/4/1964; L. Artz (JMUH)

- North of Tom's brook on \#651 about .5 mi west of \#11 (between \#11 and \#81); old overgrown woodland trail.; 5/4/1977; R.C. Simpson (LFCC)

\section{Molluginaceae}

Mollugo verticillata $\mathrm{L}$. (green carpetweed) *

- Public boat landing at N. Fork Shenandoah River, a few meters from Narrow Passage Creek.; 8/16/2011; 38.84590,-78.52963; Domangue 554

\section{Moraceae}

Broussonetia papyrifera (L.) L'Hér. ex Vent. (paper mulberry) *

- $\quad$ Rt. 663 on roadside bank. Small tree.; 8/16/2011; 38.90147,-78.48238; Domangue 562

- 1 mi. So. Strasburg, 3/4 mi, S. East of intersection of Rt. 11 on 601; Elev. 575 ft.; 8/7/1979; P. Johns (LFCC)

- $\quad$ Fort Valley, near Detrick; Elev. 1984 ft.; 10/1/1981; M. Krouse (LFCC)

Maclura pomifera (Raf.) Schneid. (osage orange) *

- $\quad 11 / 2$ mile east on Rt. 665 in the field owned by Burgess Dellinger; cow field; Elev. $808 \mathrm{ft}$;; 9/10/1979; W. Rush (LFCC)

- On Rt. 11, 1.5 miles South of Middletown where Rt. 11 crosses over Cedar Creek; floodplain; Elev. 400 ft.; 10/12/1978; R.F. Whiting (LFCC)

Morus alba L. (white mulberry) *

- Woodstock, Riverview Park Edge of lawn and forest behind baseball field. Large tree.; 5/18/2011; 38.87349,-78.49142; Domangue 228

- $\quad$ Strasburg at intersection of Rt. 11 and Rt. 81; In field at roadside sparsely wooded.; 10/11/1979; R. Bowden (LFCC)

- Rt. 601 - 1 mi. So. Strasburg 3/4 mi. S. East of intersection of Rt. 11 and 601; Found along drive way. Heavily wooded; Rocky.; Elev. 575 ft.; 8/7/1979; P. Johns (LFCC)

Morus rubra L. (red mulberry)

- Shenandoah Co. Park, beside parking lot at forest border. Tree.; 6/15/2011; 38.93673,-78.45174; Domangue 350

- turn east on 691 off of rt 42, go 5 miles on the north side of the road, on property owned by Jessie Funkhouser; Cow field bordered by tall deciuous and conifer trees; Elev. 872 ft.; 9/1/1979; W. Rush (LFCC)

- Fisher's Hill Dam; wet meadow.; 9/5/1975; R. Simpson (LFCC)

Myricaceae

Comptonia peregrina (L.) Coult. (sweet fern)

- Mass. Mountains.; 7/12/1977; R.C. Simpson (LFCC)

- Wolf Gap Rt 691; Elev. 3000 ft.; 6/9/1975; R.C. Simpson, C. Hupp (LFCC) 
Nelumbonaceae

$\diamond$ Nelumbo lutea Willd. (American lotus)

Nymphaeaceae

Nuphar advena (Aiton) W.T. Aiton (yellow pond-lily)

- $\quad$ Passage Cr.; Creek; Altitude 800.; 7/22/1965; L. Artz (JMUH)

$\diamond$ Nymphaea odorata Aiton ssp. odorata (American white waterlily)

Nyssaceae

Nyssa sylvatica Marsh. (blackgum)

- $\quad$ Tom's Brook.; 9/9/1980; R.C. Simpson (LFCC)

- $\quad$ Little Ft. Recreation Area.; 6/19/1975; R.C. Simpson (LFCC)

Oleaceae

Chionanthus virginicus L. (white fringetree)

- $\quad$ Near the intersection of Cedar Creek Rd. and Brocks Ln., on steep bank above Cedar Creek.; 4/30/2012; 39.05040,-78.44363; Domangue, M. Lantz 692

- $\quad$ Mine Run; Stream Bank; Altitude 1050 ft.; 5/20/1963; L. Artz (JMUH)

- $\quad$ King's Crossing 5.9 miles; A native of the United States, fringe tree is found from New Jersey to Oklahoma.; (JMUH)

- Woodstock Reservoir; Road side; Elev. 1,290 feet.; 5/27/1980; R.C. Simpson, S. Gwyn (LFCC)

- $\quad$ Fort Valley, rt. 678; white pine plantation.; R. Simpson (LFCC)

+ Forsythia suspensa (Thunb.) Vahl (weeping forsythia)*

- $\quad$ Woodstock, Riverview Park. Woody shrub on edge of parking lot.; 3/29/2011; 38.87434, -78.49018; Domangue 73

Fraxinus americana L. (white ash)

- $\quad$ Large tree growing a few meters from N. Fork Shenandoah River.; 5/30/2011; 38.87078, -78.49008; Domangue 287

- $\quad$ East of Edinburg.; Dry woods.; 5/18/1983; BIO 50110 (JMUH)

- Massanutten Mountain; Little Fort Recreation Area, along Powell's Mountain Trail (to Edinburg Gap) about 1 mile south of the Little Fort Camping Area; Elev. 1390 ft.; 6/26/1977; R.C. Simpson (LFCC)

Fraxinus nigra Marsh. (black ash)

- $\quad$ Little Fort; Bog.; 8/6/1964; L. Artz (JMUH)

- $\quad$ Fort Valley Bog at Powells Fort Camp growing along Little Passage Creek; Bog; Elev. 900 ft.; 8/16/1978; L. Copley, R.C. Simpson (LFCC)

- $\quad$ Powell's Fort Camp 8 miles S.E. of Elizabeth Furnace; Bog.; 6/27/1975; R. Simpson (LFCC) 
Fraxinus pennsylvanica Marsh. (green ash)

- George Washington National Forest, Elizabeth Furnace Picnic Area; field next to stream; Elev. 800 feet.; 9/20/1958; R.E. Keplinger (LFCC)

- 2 miles south of Mt. Olive on Rt. 623 on west side of road in Flat woods; Oak-Hickory forest; Elev. 1000 ft.; 10/15/1978; C. Whittington (LFCC)

+ Ligustrum obtusifolium Sieb. \& Zucc. (border privet) *

- Woodstock, Riverview Park beside Effinger Trail. Shrub.; 5/30/2011; 38.87129,-78.49004;

Domangue 288

Ligustrum sinense Lour. (Chinese privet) *

- $\quad$ Flat Rock Church, at the edge parking lot. Shrub $3 \mathrm{~m}$ tall.; 6/3/2011; 38.69995,-78.74562;

Domangue, M. Lantz 305

- Approximately 2 miles North of Woodstock off US Rt 11 at Pugh's Run; Edge of stream; Elev. 800 ft.; 6/5/1977; R. Simpson, K. Ramsey (LFCC)

\section{Onagraceae}

$\diamond$ Circaea alpina L. ssp. alpina (small enchanter's nightshade)

Circaea lutetiana L. (broadleaf enchanter's nightshade)

- $\quad$ Elizabeth Furnace Recreation Area, beside parking lot.; 6/24/2011; 38.92955,-78.32832; Domangue, J. Courtwright 381

- Woodstock; Turn off Rt. 11 and go 1 1/4 miles East on Lakeview Road and take left on old dirt road and go 3/4 mile; River bottom meadow.; 7/5/1982; J. Nutter 17 (JMUH)

$\diamond$ Epilobium angustifolium L. (fireweed)

$\diamond$ Epilobium ciliatum Raf. ssp. ciliatum (fringed willowherb)

$\diamond$ Epilobium coloratum Biehler (purpleleaf willowherb)

Gaura biennis L. (biennial beeblossom)

- In weedy lot at the intersection of rt. 11 and rt. 620.; 9/1/2011; 38.69479,-78.64812; Domangue 589

- Elizabeth Furnace Recreation Area near Passage Creek. $1 \mathrm{~m}$ tall.; 8/23/2011; 38.92922, -78.32961; Domangue 579

Ludwigia alternifolia $\mathrm{L}$. (seedbox)

- $\quad$ Tomahawk Pond Campground, growing on the edge of pond.; 7/13/2011; 38.75898,-78.84057; Domangue 443

Ludwigia palustris (L.) Elliott (marsh seedbox)

- Rt. 658; 1.3 mi N from Rt 675 NW of Edinburg; Elev. 800 ft.; 6/19/1977; G. Evans, K. Ramsey, R.C. Simpson (LFCC) 
Oenothera argillicola Mack. (shale barren evening primrose)

- $\quad .2$ mi S of Natl Forest on Co. Rt. 678 (Fort); shale barrens.; 8/8/1976; C.R. Hupp (LFCC)

- $\quad$ N end of Ft. Valley on Rt \#678 2 mi. S of White Pine plantation.; growing on west side of road. Shale barrens.; flowers yellow.; 9/17/1975; K.D. Ramsey (LFCC)

Oenothera biennis L. (common evening primrose)

- $\quad$ Rt. 730 roadside at forest border. $1.5 \mathrm{~m}$ tall.; 7/29/2011; 38.71358,-78.58472; Domangue 480

- $\quad$ 1/4 mile south Elizabeth Furnace. George Washington National Forest; roadside.; 8/21/1978; S.M. Collins (LFCC)

Oenothera fruticosa L. (narrowleaf evening primrose)

- $\quad 57.3$ mi. N.E. of J.M.U. along roadside and just into wood at location of Mudhole Bog. Very common in roadside soils and fields. June - October.; 6/22/1977; K. Kingsley 8 (JMUH)

- $\quad$ Northern Massanutten Mountains; Mudhole Bog.; 7/5/1978; J. Huffman, B. Latham, D. Stoltzfus 20 (JMUH)

- $\quad$ Peters Mill, Little Fort.; 6/20/1975; R.C. Simpson (LFCC)

- Massanutten Mountain, Little Fort Recreation Area, along Powell's Mountain trail (to Edinburg Gap) about 1 mi. South of the Little Fort Camping Area; Shale barrens.; 6/26/1977; R.C. Simpson, G. Evans (LFCC)

Oenothera fruticosa L. ssp. fruticosa (narrowleaf evening primrose)

- Elizabeth Furnace Recreation Area a couple meters from Passage Creek.; 6/24/2011; 38.92751, -78.33083; Domangue, J. Courtwright 373

$\diamond$ Oenothera laciniata Hill (cutleaf evening primrose)

+ Oenothera parviflora $\mathrm{L}$. (northern evening primrose)

- In weedy lot at the intersection of rt. 11 and rt. 620. $1.5 \mathrm{~m}$ tall.; 9/1/2011; 38.69508,-78.64852; Domangue 586

- $\quad$ Strasburg Park in weedy field. Clay soil.; 8/2/2011; 38.97549,-78.35374; Domangue 519

- $\quad$ Edinburg Gap, west side; Shale barren.; 7/13/1975; R. Simpson (LFCC)

- North end of Fort Valley, Rt \#678; 1 mile south of Blue hole; Shale barren.; 8/9/1975; R.C. Simpson (LFCC)

$\diamond$ Oenothera perennis L. (little evening primrose)

Orobanchaceae

Conopholis americana (L.) Wallr. (American cancer-root)

- $\quad$ FR 374 roadside on the edge of forest, growing on decaying wood.; 5/30/2012; 38.73039, -78.56824; Domangue 703

- $\quad$ Beside cabin. Growing in wooded area on decayed wood.; 5/11/2011; 38.95327,-78.52893; Domangue, C.K. McMullen 199

$\diamond$ Epifagus virginiana (L.) W. Barton (beechdrops)

$\diamond$ Orobanche uniflora $\mathrm{L}$. (oneflowered broomrape) 
Oxalidaceae

+ Oxalis corniculata $\mathrm{L}$. (creeping woodsorrel)

- $\quad$ Woodstock, Riverview Park. Growing in lawn beside gazebo.; 5/18/2011; 38.87339,-78.49358;

Domangue 219

Oxalis dillennii Jacq. (slender yellow woodsorrel)

- Shale barrens south of Elizabeth Furnace RT 678; Shale barrens.; 4/27/1977; C. Hupp (LFCC)

- $\quad$ 1/4 mi W. of Rt. 628 at Cedar Cr.; open meadow yellow bloom; Altitude 980 ft.; 9/28/1975;

E. Fowlkes (LFCC)

Oxalis florida Salisb.

- Hockman Homestead. One mile north of Edinburg. Just south of dwelling.; Vegetable garden.; 9/28/1975; G. Beaty (LFCC)

$\diamond$ Oxalis grandis Small (great yellow woodsorrel)

Oxalis stricta L. (common yellow oxalis)

- $\quad$ Rt. 672 roadside near N. Fork Shenandoah River.; 9/1/2011; 38.84521,-78.53076; Domangue 593

- Clearing in wooded area near small cabin.; 5/11/2011; 38.94997,-78.52692; Domangue, C.K. McMullen 185

- Devil's Backbone State Forest on the edge of dirt road in forest. Clay soil.; 5/10/2011; 39.04689, -78.43017; Domangue, B. Burkholder 175

- $\quad$ East of Edinburg on Route 678 at the junction of 675; Flood plain - roadside woods.; 5/16/1984; D. Phillips, M. Klee (JMUH)

- 1 mile north of Rt \#55 at wheatfield, Along roadside of Rt \#623, West (100 yds); Along roadside; Elev. 1000.; 8/13/1978; B. Legge (LFCC)

- 1 mile E. of Liberty Furnace at junction of rt. 717 and 690.; Shale barren.; 9/2/1975; R.C. Simpson (LFCC)

Oxalis violacea $\mathrm{L}$. (violet woodsorrel)

- Clearing in wooded area near small cabin. Purple-pink flowers.; 5/11/2011; 38.94997,-78.52692; Domangue, C.K. McMullen 184

- Elizabeth Furnace Recreation Area on the edge of lawn and weedy area near Passage Creek.; 5/4/2011; 38.92770,-78.33052; Domangue 152

- $\quad 3.0$ mi. NE of Lebanon Church on Route 628. Floodplain of stream.; Edible parts: Leaves may be eaten raw in small quantities or steeped in tea.; 5/21/1988; Cason, Gordon 17 (JMUH)

- Power line 300 yds E. of Rt. 717; 4 mi NE. of Basye; dry - moist open area - gravelly.; 5/23/1984; S. Arey $36(\mathrm{JMUH})$

- $\quad 3.0$ miles NE of Lebanon Church on Rt. 628.; 5/21/1988; Chandler 16 (JMUH)

- Approx 2 mi. N.W. of Jct. of \#675 and \#678 on \#675 on the Massanutten Mtn.; moist woodside.; 5/4/1977; R.C. Simpson (LFCC)

- $\quad$ Rt. \#678 1 mile south of Elizabeth Furnace; Growing on side of road; Elev. 725 ft.; 5/22/1978; K. Halstead (LFCC) 


\section{Papaveraceae}

Chelidonium majus L. (celandine) *

- Shenandoah Co. Park. Edge of grass trail in light forest. Plant with bright orange sap.; 5/18/2011; 38.93671,-78.45296; Domangue 236

Papaver dubium L. (blindeyes) *

- $\quad$ Fort Valley, in open field among dense grasses.; 5/4/2011; 38.88433,-78.37648; Domangue 138

- Cultivated fields, waste lands; 678, Fort Valley.; (JMUH)

- $\quad$ Strasburg, Junction of Rt. 55 and Rt. 623; Dry, grassy field; Elev. 700 ft.; 5/23/1978; R.F. Whiting (LFCC)

- Rt. \#11 at Junction with I-81; Growing on roadside; Elev. 600 ft.; 5/22/1978; K. Halstead (LFCC)

Sanguinaria canadensis L. (bloodroot)

- Shenandoah Co. Park. Near park entrance in lightly forested area.; 4/5/2011; 38.93671,-78.45324; Domangue 79

- 2 miles east of Fishers Hill along the Shenandoah River; wooded terrace of a flood plain; Elev. 900 ft.; 4/1/1978; R.C. Simpson, M. Watts (LFCC)

- Rt. 623, .7 mile north of the intersection of Rt. 623 and 714.; Broadleaf, deciduous forest.; Elev. 700 ft.; 4/24/1978; L. Frey, J. Brumback, R. Whiting (LFCC)

\section{Passifloraceae}

$\diamond$ Passiflora lutea $\mathrm{L}$. (yellow passionflower)

Paulowniaceae

$\diamond$ Paulownia tomentosa (Thunb.) Steud. (princesstree) *

Penthoraceae

Penthorum sedoides L. (ditch stonecrop)

- $\quad$ Tomahawk Pond Campground, on the edge of pond.; 8/8/2011; 38.75891,-78.84074; Domangue 527

Phrymaceae

Phryma leptostachya L. (American lopseed)

- $\quad$ Conicville, off rt. 694 in forest.; 6/30/2011; 38.83209,-78.67326; Domangue 392 
Phytolaccaceae

Phytolacca americana L. (American pokeweed)

- Growing in riparian zone at Meems Bottom Covered Bridge.; 7/12/2011; 38.72069,-78.65476;

Domangue 424

- $\quad$ FR 274 West side.; 7/22/1981; D.E. Miller 23 (JMUH)

- Near strm, 1 mile SW of Fort Valley road; Altitude 1300 feet.; 8/3/1971; C.A. Helsley (JMUH)

- $\quad$ Rt. 678 Fort Valley "Glass House."; 7/13/1975; R.C. Simpson (LFCC)

- 1 mile west of Lebanon Church on 55. 100 yards north off 55; woods, near dried up pond.; 10/11/1978; M.J. Hughes (LFCC)

\section{Plantaginaceae}

Plantago aristata Michx. (largebracted plantain) *

- $\quad$ Little Fort Recreation trail.; 6/13/1980; R.C. Simpson (LFCC)

- 1 mile north of Rt. \#717 \#770 junct.; 1/2 mile west of \#770 - \#678 junct.; 7/27/1980;

D. Poffenberger, R. Graves (LFCC)

Plantago lanceolata L. (narrowleaf plantain)*

- Cedar Grove Church. Growing in gravel parking lot.; 5/18/2011; 38.69433,-78.64742; Domangue 209

- $\quad 3.0$ miles NE of Lebanon Church on Rt. 628.; 5/21/1988; Chandler 19 (JMUH)

- $\quad 3.0$ mi. NE of Lebanon Church on Route 628. Floodplain of stream.; Edible Parts: Young leaves boiled and eaten as a potherb. Seeds dried and ground into flour or dried, boiled, and served as a hot cereal.; 5/21/1988; Cason, Gordon 19 (JMUH)

- Fisher Hill Dam; Fishers Hill, 2 mi. south of Strasburg.; 4/7/1975; K. Ramsey (LFCC)

- Hockman Homestead ("Chequers") 1 mile north of Edinburg on U.S. Route 11 on east side of road; Unmowed lawn.; 6/13/1980; G. Evans (LFCC)

Plantago major L. (common plantain) *

- Rt. 620 roadside, across from Cedar Grove Church.; 7/12/2011; 38.69439,-78.64739; Domangue 422

Plantago rugelii Dcne. (blackseed plantain)

- $\quad$ Conicville, on dirt trail in forest.; 6/30/2011; 38.83218,-78.67347; Domangue 393

- $\quad$ FR 274 east, Story Trail.; 6/24/1981; D.E. Miller 60 (JMUH)

- Hockman Homestead. 1 mile north of Edinburg U.S. Rt. 11. 25 feet west of dwelling; lawn.; 10/2/1975; G. Beaty (LFCC)

- 1 mile north of Rt \#55 at wheat field, along roadside of Rt \#623 West (100 yds); Along roadside, disturbed area; Elev. 1000.; 8/13/1978; B. Legge (LFCC)

Plantago virginica $\mathrm{L}$. (Virginia plantain)

- $\quad$ Tower road, E of Woodstock.; 5/1/1975; R.C. Simpson (LFCC) 
Platanaceae

Platanus occidentalis L. (American sycamore)

- Elizabeth Furnace Recreation Area. Large tree growing in lawn.; 5/25/2011; 38.92837,-78.33027;

Domangue 262

- $\quad$ George Washington National Forest, Elizabeth Furnace Picnic Area; Elev. 800 feet.; 9/20/1978;

R.E. Keplinger (LFCC)

- $\quad$ Strasburg inter. Of 4th St. and King St. W. (Old King St) 200 yds west down along stream's edge; edge of stream; Elev. 600 ft.; 10/13/1979; R. Bowden (LFCC)

Polemoniaceae

Phlox divaricata $\mathrm{L}$. (wild blue phlox)

- Shale bank; Ft. Valley, George Wash. Nat. Forest, on west road side bank across from picnic area Elizabeth Furnace.; Flowers: blue.; 4/20/1975; R.C. Simpson (LFCC)

Phlox maculata L. (wild sweetwilliam)

- $\quad$ Little Fort V.; Bog; Altitude 1400.; 7/29/1965; L. Artz (JMUH)

$\diamond$ Phlox ovata L. (wideflower phlox)

Phlox paniculata L. (fall phlox)

- $\quad$ Elizabeth Furnace Recreation Area, a few meters from Passage Creek. $1 \mathrm{~m}$ tall.; 7/29/2011; 38.92946,-78.32891; Domangue 492

- 1 mile E of Liberty Furnace at junction of Routes of 717 and 690. Stony Creek flood plains.; 9/7/1975; R.C. Simpson (LFCC)

Phlox subulata L. (moss phlox)

- Woodstock, Riverview Park. On rocky ledge off Effinger Trail.; 4/18/2011; 38.87205,-78.49027; Domangue, J. Courtwright 105

- $\quad$ State rte. 758, west facing slope, shale barren along road-side.; 4/12/1980; W.A. Wood 26 (JMUH)

- $\quad 3.7$ miles from E. of Rt. 11 on 675 on S.W. facing slope.; Devonian shale slope.; 4/1/1982;

C. Wagner 25 (JMUH)

- $\quad 3.7$ miles east of jct. of Rte. 11 and Edinburg on Rte. 675; shale barren.; 4/16/1982; C. Yost 9 (JMUH)

- $\quad 3.7$ mi. east of Rt. 11 and Rt. 675 junction in Edinburg on Rt. 675.; Devonian shale barren, on south facing slope.; 4/12/1982; J. Andrews 27 (JMUH)

- $\quad$ On route 675, 3.7 miles east of junction of route 11 and Edinburg.; Devonian shale-barren slope.; 4/6/1982; J. Beer (JMUH)

- $\quad 3.0$ miles north of Kings Crossing on Rt. 678 on rocky shale bank along roadsides; Shale barrens.; 4/25/1983; B. Kelley 28 (JMUH)

- $\quad 3$ miles north of Kings Crossing on 678.; East facing shale bank.; 4/25/1983; T. Rexroad 1221 (JMUH)

- $\quad 3.7$ mi east of Edinburg; West facing slope on shale barrens.; 4/12/1982; R. Alfonso 26 (JMUH)

- Rt. 628 Fort Valley; Semi-wooded area.; 4/22/1975; A. Haley, D. McDonald, S. Pence (LFCC)

- $\quad$ Fort Valley, 5.7 mile south of junction of \#55 and \#678 on 678; Shale barren.; 4/25/1980; T. McCracken (LFCC) 
Polygalaceae

Polygala paucifolia Willd. (gaywings)

- $\quad$ Laurel Run Rd.; Elev. 1100 ft.; 5/13/1980; S. Gwyn (LFCC)

- Orkney Springs, Junction of Routes 720 and 7230.1 mi. on Route 723; Roadside, wooded area.; 5/23/1981; E. Lerch (LFCC)

$\diamond$ Polygala polygama Walt. (racemed milkwort)

$\diamond$ Polygala sanguinea $\mathrm{L}$. (purple milkwort)

Polygala verticillata L. (whorled milkwort)

- $\quad$ Fort Valley; Dry, sandy soil; Altitude 1200.; 6/22/1965; L. Artz (JMUH)

- $\quad$ On Rt. \#678; Note flowers were white.; 5/31/1977; R.C. Simpson (LFCC)

\section{Polygonaceae}

Eriogonum allenii S. Wats. in A. Gray et al. (shale barren buckwheat)

- Rt 675 (Bowman's Crossing) 3.6 mi east of Edinburg left side on shale bank.; 5/16/2000;

B. Ramsey, Flora of VA Class 157 (JMUH)

- $\quad$ Rt 675 Fort Valley Edinburg; Shale barren endemic.; 5/21/1986; BIO 501 (JMUH)

- Shale barrens; 3.5 miles east from Edinburg along Rt. 675.; 6/27/1978; J. Huffman, B. Latham, D. Stoltzfus 3 (JMUH)

- $\quad$ Edinburg Gap; Shale; Altitude 1500.; 8/6/1964; L. Artz (JMUH)

- Shale Barren along Rt. 675 east of Edinburg.; Shale Barren endemic.; 7/1/1976; N.L. Bodkin (JMUH)

- Rte. 675 Bowman's Crossing, 3.5 miles from Edinburg.; sunny shale barren above roadside, endemic species.; 7/2/1979; VA Flora Class 17 (JMUH)

- $\quad$ Rt \#678 at Edinburg Gap; Shale barren.; 6/26/1975; R.C. Simpson (LFCC)

- $\quad$ Edinburg Gap.; 5/26/1980; S.M. Collins, J.A. Hepner (LFCC)

Fallopia convolvulus (L.) Á. Löve (black bindweed) *

- $\quad$ Edinburg Gap, shale barren, westside; Shale.; 7/13/1975; R.C. Simpson (LFCC)

Fallopia scandens (L.) Holub (climbing false buckwheat)

- $\quad$ Strasburg Park, on the edge of grassy field.; 9/15/2011; 38.97572,-78.35452; Domangue 628

- $\quad$ Growing beside dirt road in woods.; 7/20/2011; 39.04799,-78.43639; Domangue, C.K. McMullen 462

- 1 mile E. of Liberty Furnace at junction of Rt \#717 and 690.; Shale Barrens.; 9/7/1975; R.C. Simpson (LFCC)

Persicaria arifolia (L.) Harolds. (halberdleaf tearthumb)

- $\quad$ Fort Valley, Rt. \#678, north end, across from start of Signal Knob Trail; wet woods.; 8/9/1975;

R.C. Simpson (LFCC)

- $\quad$ Fort Valley road \#678, across from entrance of Signal Knob Trail.; Wet woods.; 8/9/1975; R.C. Simpson (LFCC)

+ Persicaria hydropiperoides (Michx.) Small (swamp smartweed)

- $\quad$ Growing in Fort Valley barnyard off rt. 678.; 10/6/2011; 38.88458,-78.37852; Domangue 654 
Persicaria lapathifolia (L.) Gray (curlytop knotweed)

- $\quad$ Strasburg Park, at boat landing beside N. Fork Shenandoah River.; 9/15/2011; 38.97337, -78.35134; Domangue 633

Persicaria longiseta (Bruijn) Kitag. (Oriental lady's thumb) *

- Growing beside gravel lot in forest off rt. 691.; 9/8/2011; 38.89235,-78.70585; Domangue 619

- $\quad$ Rt. 600 roadside.; 8/2/2011; 38.91550,-78.46568; Domangue 516

- $\quad$ Elizabeth Furnace Recreation Area, on the edge of lawn.; 6/24/2011; 38.92833,-78.33037;

Domangue, J. Courtwright 377

- Woodstock, Riverview Park. Found on grassy trail in shade.; 5/30/2011; 38.87308,-78.48867; Domangue 284

- 2.5 miles west of Columbia Furnace at junc. Of Rt. \#675 and \#717; Floodplain; Elev. 1800 ft.; 9/7/1975; R.C. Simpson (LFCC)

- Approx 1/2 mile from the Shenandoah River bridge on 55 in a field along side the river 2 miles east of Strasburg.; Shallow water.; 10/18/1978; M. Hughes (LFCC)

Persicaria maculosa Gray (spotted ladysthumb) *

- $\quad$ Growing in lawn beside gravel driveway.; 8/8/2011; 38.79531,-78.66101; Domangue 546

- Camp Roosevelt, growing in weedy gravel lot.; 6/24/2011; 38.72800,-78.51525; Domangue, J. Courtwright 364

- 6 mi West of Columbia Furnace on Laurel Run at intersection of Rt \#691 and Rt 717.; At edge of seepage pond.; 6/5/1977; R. Simpson (LFCC)

- Route 6851.3 mile north from Route 675. Northwest from Edinburg; large wet seepage pond in cow pasture; note flowers pink; Elev. 850 ft.; 6/19/1977; G. Evans, K. Ramsey (LFCC)

Persicaria pensylvanica (L.) Gomez (Pennsylvania smartweed)

- Public boat landing at N. Fork Shenandoah River, a few meters from Narrow Passage Creek.; 8/16/2011; 38.84590,-78.52963; Domangue 553

- Hockman Homestead, 1 mile north of Edinburg, U.S. Rt 11., 50 feet N.N.W. of dwelling; roadside.; 10/2/1975; G. Beaty (LFCC)

Persicaria punctata (Ell.) Small (dotted smartweed)

- 1 mile E. of Liberty Furnace at junction of Rt \#717 and 690.; 9/7/1975; R.C. Simpson (LFCC)

- NE End Edinburg, jct. Stoney Creek and Shen River, RT 675; Elev. 850 ft.; 6/19/1977; G. Evans, K. Ramsey, R.C. Simpson (LFCC)

Persicaria sagittata (L.) H. Gross (arrowleaf tearthumb)

- Devil's Backbone State Forest, on foot trail in woods.; 7/20/2011; 39.04944,-78.43409; Domangue, C.K. McMullen 466

- $\quad$ Rt 678 one mile north of intersection of Rt 675. King's Crossing.; Near pond; $50 \mathrm{ft}$ from road.; 8/3/1975; G. Beaty (LFCC)

- 6 mi west of Columbia Furnace on Laurel Run at intersection of RT \#'s 691 and 717.; Along edge of seepage pond.; 6/5/1977; R. Simpson (LFCC)

Persicaria virginiana (L.) Gaertn. (jumpseed)

- $\quad$ 1/4 mi. W. of Rt. 628 at Cedar Cr.; wooded slope, shady with old vegetation - green bud like with white; bamboo-like stem.; 9/30/1975; E. Fowlkes (LFCC) 
Polygonum aviculare L. (prostrate knotweed) *

- Rt. 685; 1.3 mi. N from Rt 675 NW of Edinburg; Elev. 850 ft.; 6/19/1977; G. Evans, K. Ramsey, R.C. Simpson (LFCC)

Polygonum tenue Michx. (pleatleaf knotweed)

- Growing in gravel lot in forest off rt. 691.; 9/8/2011; 38.89268,-78.70621; Domangue 615

- Junction of Rt 717 and Rt. 675 - West of Columbia Furnace; Shale Barrens.; 9/7/1975; R.C. Simpson (LFCC)

+ Rumex acetosella $\mathrm{L}$. (common sheep sorrel) *

- $\quad 3.0$ miles NE of Lebanon Church on Rt. 628.; 5/21/1988; Chandler 1 (JMUH)

- $\quad 3.0$ mi. NE of Lebanon Church on Route 628. Floodplain of stream.; Edible Parts: Leaves eaten sparingly in salads, boiled as a potherb, or steeped as a tea.; 5/21/1988; Cason, Gordon 20 (JMUH)

+ Rumex conglomeratus Murr. (clustered dock)*

- $\quad$ Edge of public boat landing parking lot, near N. Fork Shenandoah River. Sandy soil. $1 \mathrm{~m}$ tall.; 6/15/2011; 38.84590,-78.52994; Domangue 344

Rumex crispus L. (curly dock)*

- $\quad$ In weedy lot at rt. 11 intersection with rt. 620.; 5/30/2011; 38.69481,-78.64817; Domangue 277

- $\quad$ Rt 6851.3 mile north from 675; large wet seepage pond in cow pasture; Elev. 850 ft.; 6/19/1977; G. Evans, K. Ramsey (LFCC)

Rumex obtusifolius L. (bitter dock) *

- Growing in riparian zone at Meems Bottom Covered Bridge.; 7/12/2011; 38.72069,-78.65476; Domangue 426

- $\quad$ Northern Massanutten Mountains; Mudhole Bog.; 7/28/1978; B. Latham 40 (JMUH)

- Powells Fort Camp, 2.6 miles north of the intersection of Rt. 273 and Rt. 66, on Rt. 66, George Washington National Forest.; Broadleaf deciduous forest, seepage area.; Elev. 1200.; 4/22/1978; L. Frey (LFCC)

- Hockman Homestead ("Chequers") 1 mi. north Edinburg US Rt. 11 on east side of road.; 6/20/1977; G. Evans (LFCC) 


\section{Portulacaceae}

Claytonia virginica L. (Virginia springbeauty)

- $\quad$ Clearing in wooded area near small cabin.; 5/11/2011; 38.94997,-78.52692; Domangue, C.K. McMullen 191

- Shenandoah Co. Park, downhill from shelter 1 and 2. In wooded area among leaf litter. Dark soil. Flowers pale pink.; 3/21/2011; 38.93443,-78.45662; Domangue, J. Courtwright 68

- On North bank of Stoney Creek about 150-200 yards on the South side of Stoney Creek Rd., 1-1.5 miles East of the Rt. 42 junction (Larkin's Grocery), approximately 400 yards downstream from swinging bridge.; 4/13/2001; I. Poplar-Jeffers 4 (JMUH)

- Camp Caroline Furnace, Fort Valley (7 miles SE of Edinburg on Route 675). 100 yards south of staff house gate, 50 yards down jeep trail on east side of road.; Rich, shady soil.; 4/23/1979; B.E. Brandt 28 (JMUH)

- Fort Valley, 5.7 mile south of junction of \#55 and \#678 on \#678; Shale barren.; 4/25/1980; T. McCracken (LFCC)

- $\quad$ Fishers hill Dam. 2 mi South of Strasburg.; Growing west of bank in a deciduous woods.; 5/7/1975; Kelly (LFCC)

$\diamond$ Portulaca oleracea L. (little hogweed) *

Primulaceae

Anagallis arvensis $\mathrm{L}$. (scarlet pimpernel) *

- In weedy lot at rt. 11 intersection with rt. 620.; 5/30/2011; 38.69481,-78.64817; Domangue 278

- $\quad$ Story Trail, off FR 274 west.; 6/24/1981; D.E. Miller 67 (JMUH)

- Woodstock; Turn off Rt. 11 and go 1 1/4 miles East on Lakeview Road and take left on old dirt road and go 3/4 miles; River bottom meadow.; 7/5/1982; J. Nutter 7 (JMUH)

- Hockman Homestead east of dwelling; Rt 11 one mile north of Edinburg.; Open field.; 10/1/1975; G. Beaty (LFCC)

- $\quad$ 1/4 mi W. of Rt. 628 at Cedar Cr.; meadow; deep salmon colored bloom; Altitude $980 \mathrm{ft}$.; 9/29/1975; E. Fowlkes (LFCC)

Dodecatheon meadia L. (pride of Ohio)

- Fort Valley, beside Mine Mountain Rd. in moist, wooded environment.; 4/26/2011; 38.89643, -78.40716; Domangue, M. Lantz 121

- Mudhole Bog.; 5/18/1983; BIO 501 (JMUH)

- $\quad$ Roadside - Little Fort Valley.; 5/1/1992; (JMUH)

- Ft. Valley on Rt \#678 to Detrick, east to Milfor Gap on Rt \#758.; beside a mountain stream; flowers pinks.; 5/4/1976; K.D. Ramsey (LFCC)

- Powells Fort Bog (Massanutten Mt.); Elev. 1200 ft.; 4/27/1977; R.C. Simpson, C. Hupp (LFCC) 
Lysimachia ciliata L. (fringed loosestrife)

- In cow pasture of weeds and woody plants.; 6/30/2011; 38.79441,-78.66053; Domangue 400

- Camp Roosevelt, beside parking lot in lightly forested area.; 6/24/2011; 38.73006,-78.51707; Domangue, J. Courtwright 368

- $\quad$ FR 274 west.; 7/22/1981; D.E. Miller 13 (JMUH)

- $\quad$ Northern Massanutten Mountains; Mudhole Bog.; 7/5/1978; J. Huffman, B. Latham, D. Stoltzfus 23 (JMUH)

- Woodstock; Turn off Rt. 11 and go 1 1/4 miles East on Lakeview Road and take left on old dirt road and go 3/4 miles; River bottom meadow.; 7/5/1982; J. Nutter 9 (JMUH)

- Abandoned roadside in deciduous woods.; at Little Fort on the Massanutten Mt.; Flowers: yellow.; 6/19/1975; R.C. Simpson (LFCC)

- $\quad$ Slope to Powells bog.; 7/25/1976; C.R. Hupp (LFCC)

Lysimachia lanceolata Walt. (lanceleaf loosestrife)

- Massanutten Mountain; Little Fort Recreation Area, along Powell's Mountain Trail (to Edinburg Gap) about 1 mile south of the Little Fort Camping Area; along mountain stream (Peter's Mill Run); Elev. 1390 ft.; 6/26/1977; R.C. Simpson (LFCC)

Lysimachia nummularia $\mathrm{L}$. (creeping jenny) *

- $\quad$ Fort Valley, 1/4 mile E. of Glass house.; 6/22/1980; R.C. Simpson (LFCC)

Lysimachia quadrifolia L. (whorled yellow loosestrife)

- $\quad$ FR 374 roadside bank at forest border. Dark, silty soil.; 5/30/2012; 38.76613,-78.54652; Domangue 705

- Shale barrens; 3.5 miles east from Edinburg along Rt. 675.; 6/27/1978; J. Huffman, B. Latham, D. Stoltzfus 5 (JMUH)

- $\quad$ Power line 300 yds E. of Rt 717; 4 mi NE of Basye; dry, sandy, eroded clearing.; 5/21/1984; S. Arey 28 (JMUH)

- In a deciduous woods; Strasburg Reservoir; Flowers: yellow.; ; K. Ramsey (LFCC)

$\diamond$ Lysimachia terrestris (L.) B.S.P. (earth loosestrife)

Samolus valerandi $\mathrm{L}$. (seaside brookweed)

- 6 mi west of columbia Furnace on Laurel Run at intersection of Rt. \#'s 691 and 717; In weedy area along stream.; 6/5/1977; R. Simpson (LFCC)

Trientalis borealis Raf. (starflower)

- $\quad$ Mine Run; Moist woods; Altitude 1050 ft.; 5/20/1963; L. Artz (JMUH) 
Pyrolaceae

Chimaphila maculata (L.) Pursh (striped prince's pine)

- Camp Roosevelt along horse trail in woods. Growing at the base of a large oak tree.; 6/24/2011; 38.72881,-78.51453; Domangue, J. Courtwright 363

- Growing in forest on mountain slope among leaf litter.; 5/11/2011; 38.95324,-78.53006; Domangue, C.K. McMullen 201

- Mud hole bog.; 4/19/1977; W.B. Clark 55 (JMUH)

- Rt. 675, right on 678, left on 769, Joppa Church.; dry woods.; 3/30/1975; M. Funkhouser 23 (JMUH)

- In woods near bog at Powell's Fort Camp.; 6/22/1977; D. Laiti 10 (JMUH)

- $\quad$ Rt 551 mile out of Lebanon Church 200 yards north off 55; Rich woods.; Elev. 1000 ft.; 10/18/1978; M.J. Hughes (LFCC)

- $\quad$ Middle Mt.; 8/18/1965; M. Krouse (LFCC)

Chimaphila umbellata (L.) W. Barton (pipsissewa)

- $\quad$ Rt. 703, left on 717, woods behind 4th house on right.; dry woods.; 4/18/1975; M. Funkhouser 22 (JMUH)

- $\quad$ Fort Valley area.; 5/18/1984; BIO 501136 (JMUH)

- $\quad$ Falls Ridge; Wooded trail.; 9/24/1980; S. Gwyn (LFCC)

Pyrola americana Sweet (narrowleaf mountainmint)

- $\quad$ Little Fort V.; Bog border; Altitude 1400.; 7/8/1965; L. Artz (JMUH)

- $\quad$ On South end of the Massanutten Mt.; 6/26/1975; R.C. Simpson, K. Ramsey (LFCC)

$\diamond$ Pyrola elliptica Nutt. (waxflower shinleaf)

\section{Ranunculaceae}

$\diamond$ Aconitum uncinatum L. (southern blue monkshood)

Anemone lancifolia Pursh (mountain thimbleweed)

- $\quad$ Fort Valley, beside Mine Mountain Rd. in moist, wooded environment.; 4/26/2011; 38.89643, -78.40716; Domangue, M. Lantz 123

- $\quad$ Route 771, Powells Fort Camp.; Mudhole bog.; 4/19/1977; B. Kolankiewicz 7 (JMUH)

- $\quad$ Mud Hole Bogs at Powells Fort Camp along Rt. 771.; In woods, in moist soil.; 4/19/1977; R. Smith 70 (JMUH)

- Powells Fort Camp, Route 771, Mudhole Bog in rich soil of deciduous forest.; 4/19/1977; P. Poole 42 (JMUH)

- $\quad$ Along road in front of Powells Fort Valley 4-H Camp.; 4/19/1977; W.B. Clark 40 (JMUH)

- $\quad$ Mudhole Bog - moist soil in woods.; 4/19/1977; K. Koman 33 (JMUH)

- $\quad$ Powell's Fort Camp, Mud Hole Bog off route 771; Found along side road in dry soil.; 4/19/1977; G. Matthews 52 (JMUH)

- $\quad$ Powell's Fort Camp. Mud Hole Bog. Rich soil. Deciduous forest, along road bank.; 4/19/1977; D. Anhold (JMUH)

- $\quad$ Bog at Powells Fort.; 4/27/1977; C.R. Hupp (LFCC)

- Rt \#691, Bear Oak Prescribed Burn, near Va. - W. Va. state line; along fireline on unburned side; Elev. 3005 feet.; 5/22/1981; S. Collins (LFCC) 
$\diamond$ Anemone quinquefolia L. var. quinquefolia (wood anemone)

Anemone virginiana $\mathrm{L}$. (tall thimbleweed)

- $\quad$ Rt. 730 roadside in forest clearing.; 6/24/2011; 38.71222,-78.58757; Domangue, J. Courtwright 356

- $\quad$ Shenandoah Co. Park, in weedy clearing in woods. Clay soil.; 6/15/2011; 38.93513,-78.45619; Domangue 347

- $\quad$ FR 274 West.; 6/24/1981; D.E. Miller 56 (JMUH)

- Woodstock; Turn off Rt. 11 and go 1 1/4 miles East on Lakeview Road and take left on old dirt road and go 3/4 mile; River bottom meadow.; 7/7/1982; J. Nutter 23 (JMUH)

- Approximately 2 miles north of Woodstock off U.S. Rt. \#11 at Pugh's Run; dry, exposed, limestone outcrop.; 6/5/1977; R. \& H. Simpson (LFCC)

Aquilegia canadensis L. (red columbine)

- $\quad$ Fort Valley, on side of Boyer Road in wooded area.; 4/26/2011; 38.89222,-78.39710; Domangue, M. Lantz 127

- $\quad 3.0$ miles NE of Lebanon Church on Rte. 628.; 5/21/1988; Chandler 10 (JMUH)

- Fishers Hill Dam; Fishers Hill, 2 mi. south of Strasburg. Growing along side of road that lead to Dam.; 4/30/1975; K. Ramsey (LFCC)

- Rt \#691, Bear Oak Prescribed Burn, near Va. - W. Va. state line; along roadbank of burned area; Elev. 3005 feet.; 5/21/1981; S. Collins (LFCC) 
Caltha palustris L. (yellow marsh marigold)

- $\quad$ Fort Valley, beside Mine Mountain Rd. in moist, wooded environment.; 4/26/2011; 38.89643, -78.40716; Domangue, M. Lantz 122

- Mudhole bog in Powells Fort Camp off of Route 771.; Bog.; 4/25/1983; T. Rexroad 1229 (JMUH)

- Mudhole Bog - very wet, muddy soil in woodsy bog.; 4/19/1977; K. Koman 35 (JMUH)

- Mudhole Bog.; moist, boggy conditions.; slightly open area along drainage area.; 4/18/1977; K. Haithcock (JMUH)

- Mudhole Bog, along stream bank at spring head.; 4/12/1980; W.A. Wood 12 (JMUH)

- $\quad 4$ miles from Powell Camp on route 771.; in drainage area at base of the mountain, in the water.; 4/6/1982; J. Beer (JMUH)

- Swamps, wet meadows; 4.5 miles north of Kings Crossing on Rt. 678.; 4/25/1983; B. Shirey (JMUH)

- $\quad$ Rte. 678, 3.4 miles from Detrick, VA.; swamps, marshy soil.; 4/14/1982; C. Yost 13 (JMUH)

- $\quad$ Route 771 Powells Fort Camp Mudhole Bog; swampy soil.; 4/19/1977; B. Stanley 26 (JMUH)

- $\quad 3.0$ miles north of Kings Crossing on Rt. 678 along riverbank; Swamps and wet meadows.; 4/25/1983; B. Kelley 10 (JMUH)

- $\quad 2.5$ miles south of Detrick on Rt. 678; Rich floodplain.; 4/25/1983; M. Daniel 101 (JMUH)

- On Fort Valley Rd $10.1 \mathrm{mi} \mathrm{N}$, in creek on E side of Rd; Slow running water.; 4/12/1982; R. Atkinson 7 (JMUH)

- $\quad$ Powell's Fort Camp at Rt. 771, Mudhole Bog.; Swampy, wet area.; 4/19/1977; D. Strite 39 (JMUH)

- $\quad 3.3$ mi south of Detrick on Rt. 678; In marshy soil along Route 678.; 4/12/1982; R. Alfonso 11 (JMUH)

- $\quad$ Powell's Fort Camp Mud Hole Bog; Wet, Boggy soil. Thinly forested.; 4/19/1977; D. Anhold (JMUH)

- Mudhole bog, 4 mi. from Powell's Fort Camp junction of Rt. 678 and Rt. 771.; Swampy area.; 4/12/1982; J. Andrews 14 (JMUH)

- $\quad$ Rt. 678, 4 miles north of Kings Crossing; Swamp area.; 4/25/1983; R. Adams 8 (JMUH)

- $\quad$ Mudhole Bog; Wet, rich, soil.; 4/19/1977; B. Kolankiewicz 27 (JMUH)

- Ft. Valley Area. Along Rt. 678. 5 mi North of Kings Crossings.; wet, stream area; swampy.; 4/25/1983; Parker 7 (JMUH)

- Powell's Fort Camp, Mud Hole Bog on Route 771; very large population growing in a bog.; 4/19/1977; G. Matthews 50 (JMUH)

- 1 mile from Rt. \#42 on Rt. 675; open spring.; 4/9/1977; C.R. Hupp (LFCC)

- Powells Fort Camp (in Massanutten Mountain); Elev. 1500 ft.; 9/12/1978; R.C. Simpson (LFCC)

Cimicifuga racemosa $\mathrm{L}$. (black bugbane)

- Rt. 730 roadside in forest clearing. $1.5 \mathrm{~m}$ tall.; 6/24/2011; 38.71222,-78.58757; Domangue, J. Courtwright 360

- Woodstock; Turn off Rt. 11 and go 1 1/4 miles East on Lakeview Road and take left on old dirt road and go 3/4 miles; River bottom meadow.; 7/5/1982; J. Nutter 3 (JMUH)

- Massanutten Mountain, Fort Valley on \#678 at shale barrens around road to Glass house.; 6/26/1977; R.C. Simpson (LFCC)

- Fort Valley at Powell's Fort Camp.; 6/27/1975; R.C. Simpson (LFCC)

$\diamond$ Clematis occidentalis (Hornem.) DC. var. occidentalis (western blue virginsbower) 
Clematis terniflora DC. (sweet autumn virginsbower) *

- Shenandoah Co. Park, in clearing in woods. Fragrant.; 9/1/2011; 38.93502,-78.45589; Domangue 596

- $\quad$ Strasburg, 1st. National Bank parking lot.; 9/4/1975; R. Simpson (LFCC)

Clematis viorna $\mathrm{L}$. (vasevine)

- $\quad$ Fort Valley on Rt 678; Blue Hole, Shade Barren; Elev. 1400 ft.; 8/9/1975; R.C. Simpson (LFCC)

Clematis virginiana $\mathrm{L}$. (devil's darning needles)

- 1 mile east of Liberty Furnace at junction of Rt. 717 and 690. Stoney Creek floodplain.; 9/7/1975; R.C. Simpson, S. Alger (LFCC)

- $\quad 2.5$ mi. W. of Columbia Furnace at the junction of 675 and 717.; shale barrens.; 9/7/1975; R.C. Simpson (LFCC)

$\diamond$ Consolida ajacis (L.) Schur. (doubtful knight's-spur) *

$\diamond$ Delphinium exaltatum Ait. (tall larkspur)

$\diamond$ Delphinium tricorne Michx. (dwarf larkspur)

Hepatica americana (DC.) Ker-Gawl. (roundlobe hepatica)

- $\quad$ Mudhole Bog - moist soil in woods.; 4/19/1977; K. Koman 34 (JMUH)

- Powell's Fort Camp, Road no. 771, Mudhole bog in moist soil approximately 20 yards from the road.; 4/19/1977; P. Poole 44 (JMUH)

- Mud Hole Bogs at Powells Fort Camp along Rt. 771.; Moist soil.; 4/19/1977; R. Smith 68 (JMUH)

- $\quad$ Susie Gwyn's house in Mawrertown.; 4/13/1980; S. Gwyn (LFCC)

- $\quad$ Rt 628 Fort Valley.; Open Woods.; 4/22/1975; S. Pence, A. Haley, D. McDonald (LFCC)

Ranunculus abortivus L. (littleleaf buttercup)

- Strasburg Park, growing beside trail a few meters from N. Fork Shenandoah River.; 3/22/2012; 38.97360,-78.35233; Domangue 665

- Tomahawk Pond Campground. Growing In lightly forested picnic area.; 5/10/2011; 38.75883, -78.84087; Domangue, B. Burkholder 169

- $\quad$ Rt 11 South, Strasburg; Frontage road.; 4/13/1977; R.C. Simpson (LFCC)

Ranunculus acris L. (tall buttercup) *

- $\quad$ Field.; 5/23/1972; D.A. Shanholtz (LFCC)

Ranunculus allegheniensis Britt. (Allegheny Mountain buttercup)

- $\quad$ Power line 300 yds. E. of Rt. 717; 4 mi. NE. of Basye; shady, edge of woods.; 5/9/1984; S. Arey 7 (JMUH)

$\diamond$ Ranunculus aquatilis L. var. diffusus With.

Ranunculus bulbosus L. (St. Anthony's turnip) *

- $\quad$ Fort Valley, in open field among dense grasses.; 5/4/2011; 38.88446,-78.37576; Domangue 137

$\diamond$ Ranunculus fascicularis Muhl. ex Bigelow (early buttercup) 
Ranunculus hispidus Michx. (bristly buttercup)

- $\quad$ Rte 628 Fort Valley Roadside, semi-wooded; N. end Goerge Wash. Nat. For.; 4/22/1975;

A. Haley, D. McDonald, S. Pence (LFCC)

- Just below Camp Roosevelt in the Fort Valley at junction of \#675 and F.S. road 274. Go South on F.S. \#274 500 yds. 100 yds. back on fire road on East side; woods; rich, moist, area; Elev. 1510'.; 4/22/1978; J. Gray (LFCC)

Ranunculus hispidus Michx. var. hispidus (bristly buttercup)

- Tomahawk Pond Campground. Growing in lightly forested area.; 5/10/2011; 38.75906,-78.84145; Domangue, B. Burkholder 166

$\diamond$ Ranunculus micranthus Nutt. (rock buttercup)

$\diamond$ Ranunculus pusillus Poir. in Lamarck var. pusillus (low spearwort)

Ranunculus recurvatus Poir. (blisterwort)

- Conicville. On shady dirt path in woods.; 5/20/2011; 38.83255,-78.67323; Domangue 241

- Clearing in wooded area near small cabin.; 5/11/2011; 38.94997,-78.52692; Domangue, C.K. McMullen 187

- $\quad$ Fishers Hill; N. Slope wooded .25 mi E of US 11.; 4/28/1976; C.R. Hupp (LFCC)

Ranunculus repens $\mathrm{L}$. (creeping buttercup) *

- $\quad$ On the west side of Woodstock at route \#11.; 5/6/1977; R.C. Simpson (LFCC)

$\diamond$ Ranunculus sceleratus L. var. sceleratus (cursed buttercup)

$\diamond$ Thalictrum coriaceum (Britt.) Small (maid of the mist)

Thalictrum dioicum $\mathrm{L}$. (early meadow-rue)

- Rt. 713 roadside on weedy bank. 1 m tall.; 4/30/2012; 38.98600,-78.54141; Domangue, M. Lantz 688, 689

Thalictrum pubescens Pursh (king of the meadow)

- $\quad$ Northern Massanutten Mountains; Mudhole Bog.; 7/28/1978; B. Latham 38 (JMUH)

$\diamond$ Thalictrum revolutum DC. (waxyleaf meadow-rue) 
Thalictrum thalictroides (L.) Eames \& Boivin (rue anemone)

- Shenandoah Co. Park, between shelter 1 and 2. In wooded area at base of large tree. Dark soil.; 3/21/2011; 38.93437,-78.45627; Domangue, J. Courtwright 67

- Camp Caroline Furnace, Fort Valley (7 miles S.E. of Edinburg on Route 675); west bank of Passage Creek, by footbridge near camp lake.; Rich flood plain.; 4/3/1979; B.E. Brandt 2 (JMUH)

- Open woods; 5 mi. East Edinburg, Flood Plain Little Passage Creek.; ; B. Shirey (JMUH)

- $\quad$ Roadside forest r. 66, Powells Fort Camp.; Wooded roadside.; 4/12/1980; W.A. Wood 10 (JMUH)

- $\quad$ Route 771 Powells Fort Camp, mudhole bog.; Moist woods.; 4/19/1977; B. Kolankiewicz 34 (JMUH)

- 3 mile north of Kings Crossing on 678.; Roadside.; 4/25/1983; T. Rexroad 1233 (JMUH)

- $\quad$ Powell's Fort Camp, Mud Hole Bog.; Sphagnum Bog.; 4/19/1977; D. Anhold (JMUH)

- Powells Fort Camp, George Washington National Forest, 2.6 miles north of the intersection of Rt. 273 and Rt. 66 on Rt. 66.; Broadleaf deciduous forest.; Elev. 1200 ft.; 4/22/1978; L. Frey (LFCC)

- $\quad$ Elizabeth Furnace Campground; campground - wooded; Elev. 872.; 5/16/1981; E. Lerch (LFCC)

Trautvetteria caroliniensis (Walt.) Vail (Carolina bugbane)

- $\quad$ Northern Massanutten Mountains; Mudhole Bog.; 7/5/1978; J. Huffman, B. Latham, D. Stoltzfus 14 (JMUH)

- Mudhole Bog by Little Passage Creek in Little Fort Valley.; Growing in a boggy area in a stream.; 6/28/1972; BIO 68520 (JMUH)

- $\quad$ Rte. 771 Powell's Fort Camp, Little Fort Valley, Mud Hole Bog.; moist, partly sunny area in bog.; 7/2/1979; VA Flora Class 2 (JMUH)

Rhamnaceae

Ceanothus americanus L. (New Jersey tea)

- $\quad$ Rt. 730 roadside in forest clearing. Low, shrubby plant.; 6/24/2011; 38.71222,-78.58757; Domangue, J. Courtwright 359

- $\quad$ Growing beside dirt road. Low shrub.; 6/8/2011; 39.04814,-78.43610; Domangue 325

- Approximately 5 miles East of Edinburg then south on Rt 658 on shale barren (visible from car) on the mountain; Elev. 2300 ft.; 7/7/1978; R.C. Simpson, J. Brumback (LFCC)

- $\quad$ Eliz. Furnace Rec. Area; Dry wood lands; Altitude 800 feet.; 8/21/1966; M. Krouse (LFCC)

Rhamnus lanceolata Pursh (lanceleaf buckthorn)

- Rt. 711 roadside along fencerow. Small shrub.; 4/30/2012; 38.79384,-78.66092; Domangue, M. Lantz 685

- Strasburg Passion Theatre; Shrubby field.; ; R.C. Simpson (LFCC)

- Strasburg Quarry; limestone cedar barrens.; 6/15/1980; R.C. Simpson (LFCC)

Rosaceae

Agrimonia gryposepala Wallr. (tall hairy agrimony)

- $\quad$ Conicville, beside dirt trail in woods.; 8/2/2011; 38.83074,-78.67409; Domangue 524

- Elizabeth Furnace Recreation Area, beside grassy field at forest border.; 6/24/2011; 38.92759, -78.32863; Domangue, J. Courtwright 369

- $\quad .2 \mathrm{mi} \mathrm{S}$ of Natl Forest on Co. Rt. 678 (Fort); shale barrens.; 8/8/1976; C.R. Hupp (LFCC)

$\diamond$ Agrimonia parviflora Ait. (harvestlice) 
Agrimonia pubescens Wallr. (soft agrimony)

- $\quad$ Fort Valley, north end, near Signal Knob trail parking area.; 8/11/1975; R.C. Simpson (LFCC)

$\diamond$ Agrimonia rostellata Wallr. (beaked agrimony)

Amelanchier arborea (Michx. f.) Fernald (common serviceberry)

- Devil's Backbone State Forest. Growing on side of dirt foot trail.; 4/18/2011; 39.04897,-78.43269;

Domangue, J. Courtwright 92

- Woodstock, Riverview Park, off Effinger Trail. Growing on rock cliff, 20 foot tall tree.; 3/29/2011; 38.87204,-78.49036; Domangue 75

- $\quad 3.7$ mi east of Edinburg past junction of rt 11 and rt 675; growing on shale barrens on west facing slope.; 4/12/1982; R. Alfonso 21 (JMUH)

- Bank of Shenandoah River; 2 mi. S. of Strasburg, 1 mi. east of Fishers Hill on Capt. F. Palmers property on Shenandoah River.; 4/15/1975; K. Ramsey (LFCC)

- West of Edinburg GWNF; Little Sluice Trail.; 6/27/1980; R.C. Simpson (LFCC)

Amelanchier laevis Wieg. (Allegheny serviceberry)

- $\quad$ Little Fort Recreation trai.; 6/13/1980; R.C. Simpson

$\diamond$ Amelanchier sanguinea (Pursh) DC. var. sanguinea (roundleaf serviceberry)

$\diamond$ Amelanchier spicata (Lam.) K. Koch (running serviceberry)

$\diamond$ Aronia arbutifolia (L.) Ell. (red chokeberry)

Aronia melanocarpa (Michx.) Elliott (black chokeberry)

- $\quad$ Rt \#691, Bear Oak Prescribed Burn, near Va. - W. Va. state line; Burned area; Elev. 3005 feet.; 5/22/1981; S. Collins (LFCC)

- $\quad$ Rt 691, Burn site; Elev. 3,005 feet.; 9/13/1980; S. Gwyn, S. Collins (LFCC)

Aruncus dioicus (Walt.) Fernald (bride's feathers)

- $\quad$ Rt. 714 on roadside bank. $1 \mathrm{~m}$ tall.; 6/8/2011; 39.07265,-78.37859; Domangue 326

Crataegus calpodendron (Ehrh.) Medik. (pear hawthorn)

- turn east on 691 off of Rt. 42, go 5 miles on the north side of the road, property owned by Jessie Funkhouser; Cowfield bordered by tall deciduous and conifer trees; Elev. 872 ft.; 9/1/1979; W. Rush (LFCC)

$\diamond$ Crataegus chrysocarpa Ashe var. dodgei (Ashe) Palmer

$\diamond$ Crataegus intricata Lange (Copenhagen hawthorn)

$\diamond$ Crataegus iracunda Beadle (stolonbearing hawthorn)

$\diamond$ Crataegus macrosperma Ashe (bigfruit hawthorn)

$\diamond$ Crataegus pruinosa (Wendl. f.) K. Koch (waxyfruit hawthorn) 
Crataegus uniflora Muenchh. (dwarf hawthorn)

- Devil's Backbone State Forest on the edge of dirt road in forest. Clay soil. Small shrub.; 5/10/2011; 39.04796,-78.43324; Domangue, B. Burkholder 178

Duchesnea indica (Andr.) Focke (Indian strawberry) *

- Off rt. 720 near N. Fork Shenandoah River. Growing in lawn beside cultivated field. Silty clay soil.; 3/22/2012; 38.72065,-78.65445; Domangue 662

- $\quad 3.0$ miles NE of Lebanon Church on Rt. 628.; 5/21/1988; Chandler 2 (JMUH)

- Hockman Homestead ("Chequers") 1 mile North of Edinburg on U.S. Route 11 on East side of Road; Near river bank, area grazed by cattle; Green bracts did not extend beyond petals. Flowers yellow, fruit red.; 6/13/1977; G. Evans, J. Evans (LFCC)

- Hockman Homestead. One mile north of Edinburg on U.S. Rt. 11. North of dwelling; Lawn.; 10/25/1975; G. Beaty (LFCC)

Fragaria virginiana Duchesne (Virginia strawberry)

- Mooreland Gap Rd., at edge of woods.; 4/26/2011; 38.71054,-78.58953; Domangue, M. Lantz 110

- $\quad$ Power line 300 yds E of Rt 717; 4 mi N.E. of Basye.; 5/18/1984; S. Arey 20 (JMUH)

- Bowmans Crossing at spring near top of gap on east side; mixed oak forest, on roadside; Elev. 1600 ft.; 5/2/1978; R. Whiting, L. Frey, J. Brumback (LFCC)

- 1 mile North of Rt. \#55 at wheatfield, along roadside of R \#636 West (100 yds.); Growing along the roadside; Elev. 1000.; 8/13/1978; B. Legge (LFCC)

Geum canadense Jacq. (white avens)

- Growing on the N. Fork Shenandoah River floodplain off Black Bear Rd.; 7/20/2011; 38.90966, -78.42236; Domangue, C.K. McMullen 451

- In cow pasture of weeds and woody plants.; 6/30/2011; 38.79396,-78.65941; Domangue 398

$\diamond$ Geum virginianum L. (cream avens)

$\diamond$ Malus angustifolia (Aiton) Michx. (southern crab apple)

Malus coronaria (L.) Mill. (sweet crab apple)

- $\quad$ RT. \#11 at junction with Cedar Creek; Growing on bank of creek; Elev. 594 ft.; 5/22/1978;

K. Halstead (LFCC)

$\diamond$ Malus pumila Mill. (paradise apple)* 
Physocarpus opulifolius (L.) Maxim. (common ninebark)

- $\quad$ Fort Valley, near Passage Creek. Woody plant $1.5 \mathrm{~m}$ tall.; 6/24/2011; 38.88412,-78.37559;

Domangue, J. Courtwright 389

- Mudhole Bog by Little Passage Creek in Little Fort Valley.; Growing in a boggy area near a stream.; 6/28/1972; BIO 68528 (JMUH)

- $\quad$ Northern Massanutten Mountains; Mudhole Bog.; 6/27/1978; J. Huffman, B. Latham, D. Stoltzfus 33 (JMUH)

- $\quad$ Mud Hole Bog, Powell's Fort Valley.; Moist, rich woodlands, thickets. Very rare in VA. Twigs used by Indians for twine.; 6/22/1977; P. Graves 8 (JMUH)

- $\quad$ Rte. 771, Powell's Fort Camp, Little Fort Valley, Mud Hole Bog.; shady, moist area in bog.; 7/2/1979; VA Flora Class 7 (JMUH)

- Ft. Valley Powells Ft. Camp Bog.; 6/27/1975; R.C. Simpson (LFCC)

- $\quad$ Fort Valley River; Elev. 1000 ft.; 10/27/1974; R. Simpson, C. Fitzmaurice (LFCC)

Porteranthus trifoliatus (L.) Britt. (Bowman's root)

- $\quad 3.7$ mi. E on Rt. 675 past Edinburg; shale barren.; 5/18/1983; BIO 50195 (JMUH)

Potentilla canadensis L. (dwarf cinquefoil)

- Clearing in wooded area near small cabin.; 5/11/2011; 38.94997,-78.52692; Domangue, C.K. McMullen 194

- Devil's Backbone State Forest. Growing under cluster of pines in a forest clearing.; 4/18/2011; 39.04884,-78.43179; Domangue, J. Courtwright 91

- Power line 300 yds. E. of Rt. 717; 4 mi. NE. of Basye; dry, open, especially sparsely vegetative areas.; 5/17/1984; S. Arey 15 (JMUH)

- Powell's Fort Camp, Mud Hole Bog.; Wet, boggy soil. Thinly forested.; 4/19/1977; D. Anhold (JMUH)

- $\quad$ Fort Valley, on \#678, 4.5 mi. of \#55.; 3/17/1977; R.C. Simpson, J. Bursey (LFCC)

- Rt \#691, Bear Oak Prescribed Burn, near Va. - W. Va. state line; 300 yards north on Laurel Run Trail off Rt \#691; Elev. 3005 feet.; 5/21/1981; S. Collins (LFCC)

$\diamond$ Potentilla norvegica L. (Norwegian cinquefoil)

Potentilla recta L. (sulphur cinquefoil) *

- $\quad$ Fort Valley, in open, weedy lot. Clay soil.; 5/25/2011; 38.88446,-78.37760; Domangue 271

- Woodstock; Turn off Rt. 11 and go 1 1/4 miles East on Lakeview Road and take left on old dirt road and go 3/4 mile. River bottom meadow.; 7/7/1982; J. Nutter 22 (JMUH)

- Hockman Homestead ("Chequers") 1 mi. north Edinburg; Elev. 850 ft.; 6/21/1977; G. Evans (LFCC)

Potentilla simplex Michx. (common cinquefoil)

- Camp Roosevelt at small clearing in forest.; 5/25/2011; 38.72945,-78.51690; Domangue 256

Prunus americana Marsh. (American plum)

- $\quad 1 / 2$ mile north of Gerome on road 717; low, flat land with a stream spreading out and making a damp soggy area, land was cleared away.; A good thicket has already formed.; 10/3/1990; C. Seal (LFCC)

- $\quad$ Ft. Valley. Mud Hole Gap.; 9/19/1975; R.C. Simpson (LFCC)

$\diamond$ Prunus angustifolia Marsh. var. angustifolia (Chickasaw plum) 
$\diamond$ Prunus avium (L.) L. (sweet cherry) *

$\diamond$ Prunus cerasus L. (sour cherry) *

+ Prunus mahaleb L. (Mahaleb cherry)*

- Shenandoah Co. Park. In fragmented weedy area surrounded by grassy lawn. Small tree.;

4/18/2011; 38.93595,-78.45219; Domangue, J. Courtwright 103

$\diamond$ Prunus munsoniana $\mathrm{W}$. Wight \& Hedrick (wild goose plum) *

$\diamond$ Prunus pensylvanica L.f. var. pensylvanica (pin cherry)

Prunus serotina Ehrh. (black cherry)

- Growing along fencerow beside lawn. Large tree.; 4/30/2012; 38.79592,-78.66228; Domangue, M. Lantz 683

- Woodstock tower trail.; 5/17/1989; W. York, T. Thacker 10 (JMUH)

- $\quad$ Eliz. Fremare Rec. Area; woods; Altitude 800 feet.; 8/14/1966; M. Krouse (LFCC)

- Capon Street at Railroad tracks; woody, shrubby area; Elev. 650 ft.; 10/11/1979; R. Bowden (LFCC)

$\diamond$ Prunus virginiana $\mathrm{L}$. var. virginiana (chokecherry)

+ Pyrus communis L. (common pear) *

- In cow pasture near the fencerow along rt. 711. Medium-sized tree.; 8/8/2011; 38.79366, -78.66051; Domangue 545

Rosa canina L. (dog rose) *

- On rt. 620 roadside across from Cedar Grove Church.; 5/18/2011; 38.69433,-78.64742;

Domangue 212

Rosa carolina L. (Carolina rose)

- Devil's Backbone State Forest. Growing beside forest road.; 6/8/2011; 39.04671,-78.42933; Domangue 314

- Rt. 706 roadside beside cultivated field. Fragrant flowers.; 6/3/2011; 38.81726,-78.65349; Domangue, M. Lantz 303

- Woodstock tower trail.; 5/17/1989; W. York, T. Thacker 13 (JMUH)

- 2 miles west of King's Crossing (junction of Rtes 675 and 678) on Rte 675.; Shale barren.; 5/17/1988; Cowherd, Francisco, Thomas 27 (JMUH)

- $\quad$ Peters Mill, Little Fort.; 6/20/1975; R.C. Simpson (LFCC)

- Burn Sight; Elev. 3000'.; 5/12/1980; T. McCracken (LFCC)

$\diamond$ Rosa luciae Franchet \& Rochebrune ex Crepin * 
Rosa multiflora Thunb. ex Murr. (multiflora rose)*

- Woodstock, Riverview Park. Viny plant growing on tree. Flowers white.; 5/18/2011; 38.87339, -78.49358; Domangue 218

- $\quad 3.0$ mi. NE of Lebanon Church on Route 628. Floodplain of stream.; Edible Parts: Petals eaten right off bush, added to salads, or steeped as a tea. Pulpy rind of rose hips eaten raw. Entire hip fresh or dried steeped for tea.; 5/21/1988; Cason, Gordon 21 (JMUH)

- Strasburg at Intersection of Fourth Street and old King St. W. 50 yds down along stream; stream bank; Elev. 650 ft.; 10/20/1979; R. Bowden (LFCC)

Rosa palustris Marsh. (swamp rose)

- $\quad 2$ miles east of Strasburg on Rt. \#55. 200 ft. from River (Shenandoah); disturbed area; Elev. 850 ft.; 10/17/1978; M. Hughes (LFCC)

Rubus allegheniensis Porter (Allegheny blackberry)

- $\quad$ Under and around Woodstock Tower.; 5/17/1989; W. York, T. Thacker 15 (JMUH)

Rubus flagellaris Willd. (northern dewberry)

- $\quad$ Power line 300 yds. E. of Rt. 717; 4 mi. NE. of Basye; dry, open, sandy areas.; 5/21/1984; S. Arey 24 (JMUH)

- Massanutten Mountain .5 mi. east of Little Fort recreation area on Rt. \#758; At base of shale barrens.; 6/26/1977; R.C. Simpson, D. Steed (LFCC)

Rubus hispidus L. (bristly dewberry)

- 1 mi. south of Elizabeth Furnace; Roadside.; 5/1/1978; R. Whiting (LFCC)

- $\quad$ Little Ft. Recreation Area.; 6/19/1975; R.C. Simpson (LFCC)

$\diamond$ Rubus laciniatus Willd. (cutleaf blackberry)*

Rubus occidentalis L. (black raspberry)

- $\quad$ Shenandoah Co. Park, beside parking lot at forest border. Stems arching.; 6/15/2011; 38.93422, -78.45544; Domangue 349

$\diamond$ Rubus odoratus L. (purpleflowering raspberry)

+ Rubus pensilvanicus Poir. (Pennsylvania blackberry)

- Weedy lot near rt. 11 and rt. 620 intersection. Arching vine $\sim 1 \mathrm{~m}$ tall.; 5/9/2012; 38.69520, -78.64876; Domangue 697

- Shenandoah Co. Park. Growing among tall grasses at the edge of grass trail.; 5/18/2011; 38.93763,-78.45066; Domangue 231

Rubus phoenicolasius Maxim. (wine raspberry)*

- $\quad$ Shenandoah Co. Park. In grassy field among cedar trees.; 5/30/2011; 38.93316,-78.45297; Domangue 295

- $\quad$ Turkey Run, Rt 55; roadside.; 10/16/1975; Dendrology Class (LFCC)

$\diamond$ Spiraea alba Du Roi (white meadowsweet)

$\diamond$ Spiraea betulifolia Pallas ssp. corymbosa (Raf.) Taylor \& McBryde (shinyleaf meadowsweet) 
Rubiaceae

Cephalanthus occidentalis L. (common buttonbush)

- $\quad$ Passage Cr.; Stream Bank; Altitude 800.; 7/19/1965; L. Artz (JMUH)

Cruciata pedemontana (Bellardi) Ehrend. (piedmont bedstraw) *

- $\quad$ Shenandoah Co. Park, beside parking lot at forest border.; 6/15/2011; 38.93673,-78.45174;

Domangue 352

- $\quad$ Strasburg, Junction of Rt 55 and Rt 623; Dry grassy field; Elev. 700 ft.; 5/23/1978; R.F. Whiting (LFCC)

$\diamond$ Diodia teres Walt. (poorjoe)

+ Diodia virginiana $\mathrm{L}$. (Virginia buttonweed)

- Growing on the N. Fork Shenandoah River floodplain off Black Bear Rd.; 7/20/2011; 38.90966, -78.42236; Domangue, C.K. McMullen 457

$\diamond$ Galium anglicum Huds. (Lamarck's bedstraw) *

Galium aparine L. (stickywilly)

- On route 600 roadside on the edge of the woods.; 5/10/2011; 38.97044,-78.52255; Domangue, B. Burkholder 170

- 2 mi W of King's Crossing (junction of Rts. 675 and 678) on Rt. 675; Roadside.; 5/17/1988; Cowherd, Francisco, Thomas 23 (JMUH)

- North of Tom's brook on \#651 about 5 mi. west of \#11 (between \#11 and \#81); overgrown woodland road.; 5/4/1977; R.C. Simpson (LFCC)

- $\quad$ Strasburg, Junction of Rt. 55 and Rt. 623.; Dry grassy field; Elev. 700 ft.; 5/23/1978; R.F. Whiting (LFCC)

$\diamond$ Galium asprellum Michx. (rough bedstraw)

Galium circaezans Michx. (licorice bedstraw)

- Shenandoah Co. Park. In lightly forested area near picnic shelter.; 5/30/2011; 38.93461,-78.45563; Domangue 294

- $\quad$ Near N. entrance to Eliz. Fur. (Fort); woods.; 8/8/1976; C.R. Hupp (LFCC)

Galium concinnum Torr. \& Gray (shining bedstraw)

- Devil's Backbone State Forest beside stream.; 6/8/2011; 39.04911,-78.43460; Domangue 323

- Fort Valley, 1/4 mile E. of Glass house.; 6/22/1980; R.C. Simpson (LFCC)

Galium latifolium Michx. (purple bedstraw)

- $\quad 2.5$ miles west of Columbia Furnace at junction of Rt. \#717 and 675; Shale Barrens.; 9/7/1975;

R.C. Simpson (LFCC)

- $\quad$ Edinburg Gap shale barren, westside; Shale.; 7/13/1975; R.C. Simpson (LFCC) 
Galium mollugo L. (false baby's breath) *

- Rt. 620 roadside near intersection with rt. 11.; 10/27/2011; 38.69457,-78.64842; Domangue 661

- Cedar Grove Church, beside parking lot in weedy patch.; 6/15/2011; 38.69409,-78.64780; Domangue 330

- $\quad$ Tomahawk Pond Campground, beside dirt trail in woods.; 6/8/2011; 38.75943,-78.84088; Domangue 307

Galium obtusum Bigelow (bluntleaf bedstraw)

- $\quad$ Powell's Fort Bog.; 6/8/1980; R.C. Simpson (LFCC)

$\diamond$ Galium pilosum Ait. (hairy bedstraw)

$\diamond$ Galium triflorum Michx. (fragrant bedstraw)

Galium verum L. (Yellow Spring bedstraw) *

- Fort Valley, 1 mile North of Detrick; Elev. 940 ft.; 7/7/1978; R.C. Simpson (LFCC)

- Fort Valley, rt. 678, 3 miles south of Elizabeth Furnace Rec. Area, right side of road, edge of woods, (mowing area).; 6/21/1977; M.P. Krouse (LFCC)

Houstonia caerulea L. (azure bluet)

- Devil's Backbone State Forest. In clearing in forest, growing with grasses. Flowers pale blue.; 4/18/2011; 39.04884,-78.43179; Domangue, J. Courtwright 90

- $\quad$ Along forest service rd. 66, 2 miles south of Powells Fort Camp. Wooded road-side.; 4/12/1980; W.A. Wood 31 (JMUH)

- $\quad$ Camp Caroline Furnace, Fort Valley (7 miles S.E. of Edinburg on Route 675); west bank of camp lake.; Dry, grassy bank.; 4/3/1979; B.E. Brandt 5 (JMUH)

- Rt \#691, Bear Oak Prescribed Burn, near Va. W. Va. state line; 300 yards north on Laurel Run Trail off Rt \#691; along bank of pond; Elev. 3005 feet.; 5/21/1981; S. Collins (LFCC)

- Bear Oak Prescribed Burn, 300 yards north on Laurel Run Trail off Rt \#691; Unburned Area; Elev. 3005 feet.; 5/3/1981; S. Collins (LFCC)

Houstonia longifolia Gaertn. (longleaf summer bluet)

- Shale barrens; 3.5 miles east from Edinburg along Rt. 675.; 6/27/1978; J. Huffman, B. Latham, D. Stoltzfus 11 (JMUH)

- $\quad$ Fort Valley at Little Fort on Peter's Mill Run.; 6/20/1975; R.C. Simpson (LFCC)

- Edinburg Gap, shale barren, westside; Shale.; 7/13/1975; R.C. Simpson (LFCC)

Houstonia tenuifolia Nutt. (longleaf summer bluet)

- $\quad$ FR 374 roadside. Silty soil.; 5/25/2011; 38.72938,-78.56740; Domangue 249

- Massanutten Mountain, Little Fort Recreation Area, along Powell's Mountain Trail (to Edinburg Gap) about 1 mi. South of the Little Fort Camping Area.; Dry Meadow Roadside.; 6/26/1977; R.C. Simpson, G. Evans (LFCC)

Mitchella repens $\mathrm{L}$. (partridgeberry)

- 4 miles N. of jct 771 and 678 in boggy area alongside a little stream. On 771.; Moist woods.; 4/1/1982; C. Wagner (JMUH)

- $1 \mathrm{ml}$ from Rt 717 at Liberty Furn.; 4/9/1977; C.R. Hupp (LFCC)

- Laurel Run Road 1/2 mile west of Rt 691 on north side of road; Bog area; Elev. 1,900 feet.; 9/30/1980; S. Gwyn (LFCC) 
Rutaceae

Ptelea trifoliata L. (common hoptree)

- Elizabeth Furnace Recreation Area, on the edge of an overgrown dirt trail. Low tree.; 7/29/2011; 38.92710,-78.32986; Domangue 490

- Rte. 678 Fort Valley.; along roadside, partly shaded area.; 7/2/1979; VA Flora Class 9 (JMUH)

- On Rt. 758 at the bottom of the mountain down the path beside the Leisure Point Sign; Tall deciduous trees; Elev. 800 Ft.; 7/4/1979; W. Rush (LFCC)

- $\quad$ Ft Valley on Rt 678 at "Glass House."; 7/18/1975; R.C. Simpson (LFCC)

$\diamond$ Zanthoxylum americanum Mill. (common pricklyash)

\section{Salicaceae}

Populus alba L. (white poplar)*

- $\quad$ Little Fort Recreation Area off Rt \#758.; Elev. 1400 ft.; 10/8/1981; J.A. Hepner (LFCC)

- Strasburg at Standard Quarry on Rt \#55 W.; under a electric/power line.; 6/17/1975; R.C. Simpson, C. Hupp (LFCC)

Populus deltoides Bartram ex Marsh. (eastern cottonwood)

- 6/28/1987; Steve Orndorff (BDWR)

- Cedar Creek Wayside, Rt. 114 mi. N. of Strasburg on Rt. 11; Flood Plain.; 10/2/1975; R. Brown, G. Suski (LFCC)

- 2 miles south of Mt. Olive on Rt. 623 on west side of road in flat woods. Found near a dry creekbed; Oak-Hickory forest; Elev. 1000 ft.; 10/15/1978; C. Whittington (LFCC)

Populus grandidentata Michx. (bigtooth aspen)

- Weedy lot near rt. 11 and rt. 620 intersection. Young tree.; 5/9/2012; 38.69504,-78.64842; Domangue 700

- At Little Fort Recreation Area in Little Fort on the Massanutten Mt.; 6/19/1975; R.C. Simpson (LFCC)

- $\quad$ Little Ft. Recreation Area.; 6/19/1975; R.C. Simpson (LFCC)

Populus tremuloides Michx. (quaking aspen)

- Woodstock Reservoir.; 5/27/1980; R.C. Simpson (LFCC)

- Woodstock Reservoir.; 5/27/1980; R.C. Simpson, S. Collins, S. Gwyn (LFCC)

Salix alba L. (white willow)*

- Cedar Creek Wayside Rt. 114 m. N. of Strasburg; Flood Plain.; 10/2/1975; R. Brown, R. Crowder (LFCC)

Salix babylonica $\mathrm{L}$. (weeping willow) *

- Beside Pleasant View Rd. along fencerow. Large tree with weeping habit.; 4/30/2012; 38.78762, -78.66546; Domangue, M. Lantz 682

$\diamond$ Salix caprea L. (goat willow) * 
Salix caroliniana Michx. (coastal plain willow)

- $\quad$ End of Water Street at N.E. end of Edinburg; Jct. Stoney Creek and Shen River; Elev. 850 ft.; 6/19/1977; G. Evans, K. Ramsey, R.C. Simpson (LFCC)

$\diamond$ Salix eriocephala Michx. (Missouri River willow)

Salix humilis Marsh. (prairie willow)

- $\quad$ Fort Valley; Peter's Mill Bog.; 6/24/1980; R.C. Simpson (LFCC)

- Peter Mill Trail, Fort Valley.; 6/16/1978; R.C. Simpson (LFCC)

Salix nigra Marsh. (black willow)

- Public boat landing. Shrubby tree growing at the confluence of Narrow Passage Creek and N. Fork Shenandoah River.; 5/9/2012; 38.84581,-78.52927; Domangue 701

- Fort Valley, growing on bank of Passage Creek at rt. 678 and rt. 775 intersection. Small tree.; 4/19/2012; 38.81203,-78.45015; Domangue 678

- Approximately 2 miles North of Woodstock off US Rt. 11 at Pugh's Run; over limestone at stream's edge.; R.C. Simpson, K.D. Ramsey (LFCC)

- $\quad$ Elizabeth Furnace Recreation Area; moist woods; Elev. 800'.; 8/3/1965; M. Krouse (LFCC)

$\diamond$ Salix occidentalis Walter (prairie willow)

Salix sericea Marsh. (silky willow)

- Growing in cow pasture beside stream. Small tree.; 4/30/2012; 38.79442,-78.65988; Domangue, M. Lantz 684

- $\quad$ Peter Mill Bog, Fort Valley.; 6/11/1978; R.C. Simpson (LFCC)

- $\quad$ Seven Fountains, Rt. \#770 - 771; Elev. Approx. 1450 ft.; 6/24/1980; R.C. Simpson (LFCC)

Santalaceae

Comandra umbellata (L.) Nutt. (bastard toadflax)

- Devil's Backbone State Forest on the edge of dirt road in forest. Clay soil.; 5/10/2011; 39.04732, -78.43137; Domangue, B. Burkholder 176

- $\quad$ Power line 300 yds. E of 717; 4 mi NE of Bayse; moist open area.; 5/23/1984; S. Arey 33 (JMUH)

- Rt \#691, Bear Oak Prescribed Burn, near Va. - W. Va. state line; along roadbank of burned area; Elev. 3005 feet.; 5/22/1981; S. Collins (LFCC)

- $\quad 11 / 2$ mi. E. of Strasburg on Reservoir Road.; mountain roadside in deciduous woods.; 4/29/1977; R.C. Simpson (LFCC)

Saururaceae

$\diamond$ Saururus cernuus L. (lizard's tail)

\section{Saxifragaceae}

Chrysosplenium americanum Schwein. ex Hook. (American golden saxifrage)

- $\quad$ Little Fort; Bog.; 8/6/1964; L. Artz (JMUH) 
Heuchera americana L. (American alumroot)

- $\quad$ FR 374 roadside bank at forest border. Dark, silty soil.; 5/30/2012; 38.78378,-78.53439; Domangue 707

$\diamond$ Heuchera pubescens Pursh (downy alumroot)

Micranthes virginiensis (Michx.) Small (early saxifrage)

- $\quad$ Clearing in wooded area near small cabin.; 5/11/2011; 38.94997,-78.52692; Domangue, C.K. McMullen 190

- $\quad$ Woodstock, Riverview Park. On edge of Effinger Trail, rocky soil.; 3/29/2011; 38.87135, -78.49023; Domangue 69

- $\quad 3$ miles north of Kings Crossing on 678.; East facing shale bank.; 4/25/1983; T. Rexroad 1222 (JMUH)

- 4 miles N. of Kings Crossing area. Along Rt 678 Ft. Valley area.; rocky hillside, facing east.; 4/25/1983; Parker 5 (JMUH)

- $\quad$ On route 675, 3.7 miles east of the junction of route 11 and Edinburg.; Devonian shale-barren slope.; 4/6/1982; J. Beer (JMUH)

- $\quad 14.6$ miles on Rte 678 from junction of Rtes 678 and 675 (King's Crossing) - turned left on 678 from 675 from 675 E. Roadside.; 5/17/1988; Cowherd, Francisco, Thomas 31 (JMUH)

- $\quad$ Fishers Hill Dam; Fishers Hill, 2 mi. south of Strasburg. Growing on west side bank in deciduous woods.; 5/7/1975; K. Ramsey (LFCC)

- 1 mile from Rt. 42 on Rt. 675; spring fed pond.; 4/9/1977; C.R. Hupp (LFCC)

Mitella diphylla $\mathrm{L}$. (twoleaf miterwort)

- $\quad$ Fort Valley, beside Mine Mountain Rd. in moist, wooded environment.; 4/26/2011; 38.89643, -78.40716; Domangue, M. Lantz 126

- fort Lee Mud Hole Bog; Found in the woods approaching the bog.; 6/22/1977; S.K. Reynolds 5 (JMUH)

- Approx 6 miles west of Columbia Furnace on Laurel Run at the intersection of Rt. 691 and Rt. 717.2 miles west on Rt. 691; on rocks in seepage area in cove forest.; 6/5/1977; R.C. Simpson, K.D. Ramsey (LFCC)

- $\quad$ Bog at Powells Fort.; 4/27/1977; C.R. Hupp (LFCC)

$$
\text { Scrophulariaceae }
$$

$\diamond$ Agalinis purpurea (L.) Pennell (purple false foxglove)

$\diamond$ Agalinis tenuifolia (Vahl) Raf. var. tenuifolia (slenderleaf false foxglove)

$\diamond$ Aureolaria flava (L.) Farw. (smooth yellow false foxglove)

$\diamond$ Aureolaria laevigata (Raf.) Raf. (entireleaf yellow false foxglove)

Aureolaria pedicularia (L.) Raf. (fernleaf yellow false foxglove)

- $\quad$ 1/2 mile S of Wolf Gap on Rt 675; Wooded Roadside.; 9/7/1975; R.C. Simpson (LFCC)

- Powells Camp Road, Fort Valley.; 6/27/1975; R.C. Simpson (LFCC)

$\diamond$ Chaenorrhinum minus (L.) Lange (dwarf snapdragon) * 
Chelone glabra L. (white turtlehead)

- $\quad$ Rt. 678 roadside (Fort Valley) underneath bridge beside small stream.; 10/6/2011; 38.88407, -78.37685; Domangue 655

- $\quad$ Little Fort; Bog, near Little Passage Cr.; Altitude 1000 ft.; 9/13/1963; L. Artz (JMUH)

- $\quad$ Mud Hole Gap.; 9/19/1975; R.C. Simpson (LFCC)

- Powell's Fort Organization Camp (in Massanutten Mt.); Elev. 1500 ft.; 9/12/1978; R.C. Simpson (LFCC)

$\diamond$ Kickxia elatine (L.) Dumort. (sharpleaf cancerwort) *

Linaria vulgaris Mill. (butter and eggs) *

- 2 miles east of Strasburg on Route \#55; under apple tree 150 feet from Shenandoah River in field; Elev. 850 ft.; 10/23/1978; M. Hughes (LFCC)

Lindernia dubia (L.) Pennell var. anagallidea (Michx.) Cooperr. (yellowseed false pimpernel)

- $\quad$ Rt. 658, 1.3 mi. N from Rt. 675, (NW Edinburg); Elev. 850 ft.; 6/19/1977; G. Evans, K. Ramsey, R.C. Simpson (LFCC)

Melampyrum lineare Desr. (narrowleaf cowwheat)

- $\quad$ FR 274 west.; 7/25/1981; D.E. Miller 30 (JMUH)

$\diamond$ Mimulus alatus Aiton (sharpwing monkeyflower)

Mimulus ringens L. (Allegheny monkeyflower)

- Tomahawk Pond Campground, growing on the edge of pond.; 7/13/2011; 38.75891,-78.84059;

Domangue 440

Pedicularis canadensis L. (Canadian lousewort)

- $\quad$ Power line 300 yds E. of Rt. 717; 4 mi NE of Basye; moist, flat grassy area.; 5/9/1984; S. Arey 8 (JMUH)

- $\quad$ Elizabeth Furnace Group Grampground Site C, beside Passage Creek.; 4/24/1985; M. Kelly (LFCC)

Pedicularis lanceolata Michx. (swamp lousewort)

- $\quad$ Little Fort V.; Bog border; Altitude 1400.; 9/6/1965; L. Artz (JMUH)

Penstemon canescens (Britt.) Britt. (eastern gray beardtongue)

- $\quad$ Growing on steep bank beside dirt road. Flowers pale pink.; 5/10/2011; 39.04831,-78.43614;

Domangue, B. Burkholder 181

- $\quad$ Blue Hole trail, Elizabeth furnace; Woods.; 5/16/1982; L. Michael Hill 5 (BDWR)

- Shale barrens; 3.5 miles east from Edinburg along Rt. 675.; 6/27/1978; J. Huffman, B. Latham, D. Stoltzfus 7 (JMUH)

- $\quad$ Fort Valley; Shale Banks.; 5/16/1964; L. Artz (JMUH)

- $\quad$ Roadside; Flower - Blue-violet.; 5/16/1975; K.C. Clarke (LFCC)

- $\quad$ Rt. \#678 1 mile south of Elizabeth Furnace; Growing on side of road next to a freshwater stream; Elev. 725 ft.; 5/22/1978; K. Halstead (LFCC)

+ Penstemon digitalis Nutt. ex Sims (foxglove beardtongue)

- $\quad$ Rt. 749 roadside in grassy clearing.; 6/8/2011; 38.90468,-78.66091; Domangue 312 
Penstemon laevigatus Ait. (eastern smooth beardtongue)

- Shenandoah Co. Park. In woody area beside road. Clay soil.; 5/30/2011; 38.93518,-78.45620; Domangue 293

$\diamond$ Penstemon pallidus Small (pale beardtongue)

$\diamond$ Scrophularia lanceolata Pursh (lanceleaf figwort)

$\diamond$ Scrophularia marilandica L. (carpenter's square)

Verbascum blattaria L. (moth mullein) *

- Rt. 620 roadside, adjacent open field.; 6/15/2011; 38.69464,-78.64809; Domangue 331

Verbascum phlomoides L. (orange mullein) *

- 2 miles east of Strasburg on Rt \#55. 50 yards from Shenandoah.; roadsides, poor fields, wastelands; Elev. 850.; 10/10/1978; M. Hughes (LFCC)

- $\quad$ Along Route 622 near Valley View Orchard, Strasburg; Open roadside; Elev. 800'.; 8/25/1978; B. Rinker (LFCC)

Verbascum thapsus L. (common mullein) *

- Rt. 730 roadside in forest clearing. $1 \mathrm{~m}$ tall.; 6/24/2011; 38.71222,-78.58757; Domangue, J. Courtwright 353

- $\quad$ FR 274 East.; 7/8/1981; D.E. Miller 47 (JMUH)

$\diamond$ Veronica americana Schwein. ex Benth. (American speedwell)

$\diamond$ Veronica anagallis-aquatica $\mathrm{L}$. (water speedwell) *

Veronica arvensis L. (corn speedwell) *

- Clearing in wooded area near small cabin.; 5/11/2011; 38.94997,-78.52692; Domangue, C.K. McMullen 182

- Fort Valley. Growing at the edge of a gravel lot in clay soil.; 5/4/2011; 38.88452,-78.37760; Domangue 143

- $\quad$ Near Strasburg, junct. Rt. 11 and I81 on Frontage Road; mustard field; Elev. 640 ft.; 4/17/1977; (LFCC)

Veronica hederifolia $\mathrm{L}$. (ivyleaf speedwell) *

- Posey Hollow Rd. Rt. 744; flood plain - south facing slope.; 4/15/1975; S. Miller, S. Henderson, J. Ott (LFCC)

- $\quad$ North facing slope south of Strasburg on Wildflower Hill.; rich, moist oak-hickory forest.; Blue flower.; 4/1/1975; M. Bowles, K. Clarke, D. Smith, G. Smith (LFCC)

Veronica officinalis L. (common gypsyweed)

- Clearing in wooded area near small cabin.; 5/11/2011; 38.94997,-78.52692; Domangue, C.K. McMullen 183

- Laurel Run Road; 1/2 mile west from intersection with Rt 691 on north side of road; Pink flower; Elev. 1,300 feet.; 5/27/1980; R.C. Simpson, S. Gwyn (LFCC) 
Veronica persica Poir. (birdeye speedwell) *

- Mt. Jackson Park, western end of Wunder St., on edge of gravel lot.; 3/21/2011; 38.75015,78.64692; Domangue, J. Courtwright 63

- On North bank of Stoney Creek about 150 - 200 yards on the South side of Stoney Creek Rd., 1 1.5 miles East of the Rt. 42 junction (Larkin's Grocery), approximately $1 / 4$ miles downstream from swinging bridge.; 4/13/2001; I. Poplar-Jeffers 3 (JMUH)

+ Veronica serpyllifolia $\mathrm{L}$. (thymeleaf speedwell) *

- Woodstock, Riverview Park. Growing in lawn and forming groundcover.; 4/11/2012; 38.87371, -78.49301; Domangue 672

- Elizabeth Furnace Recreation Area on grassy path in lightly forested area.; 5/4/2011; 38.92898, -78.32979; Domangue 153

$\diamond$ Veronicastrum virginicum (L.) Farw. (Culver's root)

Simaroubaceae

Ailanthus altissima (Mill.) Swingle (tree of heaven) *

- $\quad$ Large tree growing in weedy lot at the intersection of rt. 11 and rt. 620;8/2/2011;38.69515, -78.64870; Domangue 499

- 6 miles S.E. of Strasburg, Va at end of River bend Rd. in Deer Rapids; Elev. 300 ft.; 9/27/1984; T.C.F. (LFCC)

- Strasburg on Rt \#50 W at Standard Quarry; under a power line.; 6/17/1975; R.C. Simpson, C. Hupp (LFCC)

Solanaceae

Datura stramonium L. (jimsonweed) *

- Public boat landing at N. Fork Shenandoah River, a few meters from Narrow Passage Creek.; 8/2/2011; 38.84594,-78.52970; Domangue 511

$\diamond$ Nicandra physalodes (L.) Gaertner (apple of Peru)*

$\diamond$ Physalis heterophylla Nees (clammy groundcherry)

Physalis longifolia Nutt. (longleaf groundcherry)

- Public boat landing at N. Fork Shenandoah River, a few meters from Narrow Passage Creek.; 8/2/2011; 38.84594,-78.52970; Domangue 512

- $\quad$ Rt. 11 roadside along fencerow.; 7/20/2011; 38.66240,-78.66306; Domangue, C.K. McMullen 445

- Shenandoah Co. Park, in overgrown, grassy field.; 7/12/2011; 38.93623,-78.45219; Domangue 431

$\diamond$ Physalis virginiana Mill. (Virginia groundcherry) 
Solanum carolinense L. (Carolina horsenettle)

- Growing on the N. Fork Shenandoah River floodplain off Black Bear Rd.; 7/20/2011; 38.90966, -78.42236; Domangue, C.K. McMullen 454

- Cedar Grove Church, beside parking lot in weedy patch.; 6/15/2011; 38.69409,-78.64780; Domangue 329

- Massanutten Mountain, Little Fort Recreation Area, along Powell's Mountain trail (to Edinburg Gap) about 1 mi South of the Little Fort camping area; moist meadow.; 6/26/1977; R.C. Simpson, G. Evans (LFCC)

- $\quad$ Rt. 648 along side of road at Sandy Hook, Strasburg.; 10/22/1978; M.L. Snarr (LFCC)

Solanum dulcamara $\mathrm{L}$. (climbing nightshade) *

- Growing on the edge of weedy lot near Flat Rock Church. Stem low. Strong odor.; 5/9/2012; 38.70028,-78.74581; Domangue 693

Solanum ptycanthum Dunal (West Indian nightshade)

- Below Meems Bottom Covered Bridge at N. Fork Shenandoah River.; 8/16/2011; 38.72073, -78.65482; Domangue 550

- Growing on the N. Fork Shenandoah River floodplain off Black Bear Rd.; 7/20/2011; 38.90966, -78.42236; Domangue, C.K. McMullen 459

- $\quad$ 1/4 mi W. of Rt. 628 at Cedar Cr.; creek bank; Altitude 800 ft.; 9/29/1975; E. Fowlkes (LFCC)

$\diamond$ Solanum rostratum Dunal (buffalobur nightshade) *

\section{Staphyleaceae}

Staphylea trifolia $\mathrm{L}$. (American bladdernut)

- Public boat landing at N. Fork Shenandoah River. Shrub.; 5/18/2011; 38.84583,-78.52956; Domangue 215

- Fort Valley, growing in weedy area near small stream. Small shrub.; 5/4/2011; 38.88414, -78.37567; Domangue 146

- $\quad$ Shenandoah R.; Stream bank; Altitude 950.; 6/4/1964; L. Artz (JMUH)

- $\quad$ Rt. 55; 2 mis. South of 55 on fish hatchery road.; along roadside by creek.; 10/14/1979; J. Evans (LFCC)

Tiliaceae

Tilia americana L. var. heterophylla (Vent.) Loud. (American basswood)

- $\quad$ Woodstock, Riverview Park beside Effinger Trail. Large tree.; 5/30/2011; 38.87160,-78.49010; Domangue 290

- $\quad$ Shen. R.; Stream bank; Altitude 950.; 6/4/1964; L. Artz (JMUH)

- Approx. 2 mi. N.W. of the Junction of \#675 and \#678 on the Massanutten Mtn.; Rich woods.; 5/4/1977; R.C. Simpson (LFCC)

Tilia americana L. (American basswood)

- George Washington National Forest; Woodstock Tower.; 10/3/1979; (LFCC)

Ulmaceae

$\diamond$ Celtis laevigata Willd. (sugarberry) 
Celtis occidentalis L. (common hackberry)

- Shenandoah Co Park. Large tree growing between lawn and forested area.; 5/18/2011; 38.93691, -78.45180; Domangue 232

- $\quad$ Milford Gap, Fort Valley; Pine stand.; 9/20/1975; R.C. Simpson (LFCC)

Celtis tenuifolia Nutt. (dwarf hackberry)

- Rte. 678 Fort Valley, Shale Barren.; dry shale barren, in bright sunlight.; 7/2/1979; VA Flora Class 11 (JMUH)

- On Rt. 758 at the foot of the mountain down the path beside the Leisure Point Sign; tall deciduous tree; Elev. 800 ft.; 7/4/1979; W. Rush (LFCC)

Ulmus americana $\mathrm{L}$. (American elm)

- $\quad$ Rt 11 and Rt 627 interesection, Middletown; 3.5 mi. go left .2 mi to house; Elev. 760.; 9/29/1979; P. Johns (LFCC)

- East of Strasburg on route 678, north end of Massanutten Mt. in Fort Valley at Elizabeth Furnace Campground.; Growing in understory of Oak hickory forest.; Elev. 800'.; 10/3/1977; O. Bate (LFCC)

Ulmus rubra Muhl. (slippery elm)

- Woodstock, Riverview Park beside Effinger Trail. Medium-sized tree.; 5/30/2012; 38.87126, -78.49003; Domangue 711

- Palmers Prop., Fisher's Hill.; 5/16/1974; R.C. Simpson (LFCC)

- $\quad$ Strasburg town limits, 150 yds W. of rt. 55.; 10/28/1979; R. Bowden (LFCC)

Urticaceae

Boehmeria cylindrica (L.) Sw. (smallspike false nettle)

- $\quad$ Woodstock, Riverview Park, near N. Fork Shenandoah River.; 8/16/2011; 38.87071,-78.48996; Domangue 561

- Devil's Backbone State Forest, on foot trail in woods.; 7/20/2011; 39.04944,-78.43409; Domangue, C.K. McMullen 465

Laportea canadensis (L.) Weddell (Canadian woodnettle)

- Growing at Meems Bottom Covered Bridge, a few meters from N. Fork Shenandoah River.; 9/1/2011; 38.72089,-78.65485; Domangue 590

$\diamond$ Parietaria pensylvanica Muhl. ex Willd. (Pennsylvania pellitory)

$\diamond$ Pilea fontana (Lunell) Rydb. (lesser clearweed)

$\diamond$ Pilea pumila (L.) Gray (Canadian clearweed)

Urtica dioica $\mathrm{L}$. (stinging nettle)

- Growing on the N. Fork Shenandoah River floodplain off Black Bear Rd.; 7/20/2011; 38.90966, -78.42236; Domangue, C.K. McMullen 453

- $\quad$ Rt. 826 roadside, on weedy bank beside cultivated field. $1 \mathrm{~m}$ tall.; 6/15/2011; 38.69292, -78.63589; Domangue 339 


\section{Valerianaceae}

Valerianella locusta (L.) Lat. (Lewiston cornsalad) *

- Fort Valley, in open field among dense grasses. Flowers pale blue.; 4/26/2011; 38.88433,

-78.37682; Domangue, M. Lantz 130

\section{Verbenaceae}

+ Phyla lanceolata (Michx.) Greene (lanceleaf fogfruit)

- $\quad$ Strasburg Park, in ditch beside parking lot.; 7/12/2011; 38.97360,-78.35113; Domangue 432

$\diamond$ Verbena scabra Vahl (sandpaper vervain)

Verbena simplex Lehm. (narrowleaf vervain)

- $\quad$ Andrew Zirkle Grist Mill parking lot in Forestville. Gravelly soil.; 5/9/2012; 38.71448,-78.72182; Domangue 696

- $\quad$ FR 374 roadside. Silty soil.; 6/24/2011; 38.72910,-78.56737; Domangue, J. Courtwright 361

- $\quad$ FR 374 roadside. Silty soil.; 5/25/2011; 38.72938,-78.56740; Domangue 250

- Elizabeth Furnace. Pull - off on west side of Rt. \#648.; 6/8/1980; R.C. Simpson (LFCC)

Verbena urticifolia $\mathrm{L}$. (white vervain)

- $\quad$ Devil's Backbone State Forest, beside dirt road.; 7/20/2011; 39.04836,-78.43542; Domangue, C.K. McMullen 470

- $\quad$ Strasburg Park, growing beside trail a few meters from N. Fork Shenandoah River.; 7/12/2011; 38.97363,-78.35220; Domangue 435

Violaceae

Viola affinis Le Conte (sand violet)

- In cow pasture of weeds and woody plants.; 4/11/2012; 38.79447,-78.66057; Domangue 670

Viola arvensis Murr. (European field pansy) *

- Mt. Jackson Park, western end of Wunder St., on slope next to tennis courts (mowed lawn).; 3/21/2011; 38.75048,-78.64680; Domangue, J. Courtwright 66

Viola bicolor Pursh (field pansy)

- Growing on steep bank beside dirt road. Clay soil.; 4/18/2011; 39.04820,-78.43597; Domangue, J. Courtwright 97

- $\quad$ State rt. 678, south of Elizabeth Furnace.; abundant along roadside.; 4/13/1980; W.A. Wood 23 (JMUH)

- $\quad$ Little Sluice Mountain trail.; 5/6/1980; R.C. Simpson (LFCC)

- Rte 628 Fort Valley; North end George Washington Nat. For.; 4/22/1975; A. Haley, S. Pence, D. McDonald (LFCC)

Viola blanda Willd. (sweet white violet)

- $\quad$ Little Sluice Mountain Trail.; 5/6/1980; R.C. Simpson (LFCC) 
Viola canadensis L. (Canadian white violet)

- $\quad$ Approximately 2 miles North of Woodstock off US rt. 11 at Pugh's Run; shaded limestone bank; Elev. 800 ft.; 6/5/1977; R. Simpson, K. Ramsey (LFCC)

Viola cucullata Ait. (marsh blue violet)

- Meems Bottom Covered Bridge near N. Fork Shenandoah River, 0.5 miles west on Wissler Road.; 4/18/2011; 38.72092,-78.65472; Domangue, J. Courtwright 88

- Woodstock, Riverview Park. On banks of detention pool behind baseball field.; 4/5/2011; 38.87339,-78.49155; Domangue 78

- $\quad$ Power line 300 yds. E. of Rt. 717; 4 mi NE of Basye; wet, fertile, creek-side.; 5/9/1984; S. Arey 3 (JMUH)

- $\quad$ Little Sluice Mountain Trail; flower purple.; 5/6/1980; R.C. Simpson (LFCC)

- Fishers Hill Dam; Fishers Hill .2 mi south of Strasburg. Growing in grass along side of road that leads to the Dam.; 4/30/1975; K. Ramsey (LFCC)

Viola fimbriatula J.E. Smith (arrowleaf violet)

- Devil's Backbone State Forest. Edge of dirt road. Rocky soil.; 4/18/2011; 39.04799,-78.43493; Domangue, J. Courtwright 95

- $\quad$ Power line 300 yds. E of Rt. 717; 4 mi NE of Basye; dry, sandy, open areas.; 5/9/1984; S. Arey 5 (JMUH)

- Approx. 2 mi. N.W. of the Junction of \#675 and \#678 on \#675; on the Massanutten Mtn.; Shale Barrens.; 5/4/1977; R.C. Simpson (LFCC)

- Rt \#691, Bear Oak Prescribed Burn, near Va. - W. Va. state line; along roadband on burned area; Flower - purple; Elev. 3005 feet.; 5/21/1981; S. Collins (LFCC)

Viola hirsutula Brainerd (southern woodland violet)

- Mt. Jackson; shale mountain.; 5/1983; J. Kauffman (HAVI)

- $\quad$ Little Sluice Mountain Trail; flower white.; 5/6/1980; R.C. Simpson (LFCC)

Viola labradorica Schrank (alpine violet)

- $\quad$ Mine Run; Moist woods; Altitude 1000 ft.; 5/20/1963; L. Artz (JMUH)

Viola macloskeyi Lloyd ssp. pallens (Banks ex DC) M.S. Baker (small white violet)

- Public park near Lake Laura. Growing in shaded lawn near stream.; 4/11/2011; 38.80500, -78.78926; Domangue 84

- $\quad$ Little Stony Creek trail; Elev. 2100 ft.; 4/20/1980; S. Gwyn (LFCC)

Viola palmata $\mathrm{L}$.

- Clearing in wooded area near small cabin.; 5/11/2011; 38.94997,-78.52692; Domangue, C.K. McMullen 197

- Camp Roosevelt, along horse trail in wooded area.; 4/26/2011; 38.73141,-78.51154; Domangue, M. Lantz 112

- Mt. Jackson; shale mountain.; 5/1983; J. Kauffman (HAVI)

- $\quad$ Little Sluice Mountain Trail; flower purple.; 5/6/1980; R.C. Simpson (LFCC)

- Flood plain on the Shenandoah River.; flowers: purple.; 4/15/1975; R.C. Simpson (LFCC) 
Viola pedata $\mathrm{L}$. (birdfoot violet)

- Devil's Backbone State Forest. Edge of dirt road. Rocky soil.; 4/18/2011; 39.04799,-78.43493;

Domangue, J. Courtwright 93, 94

- West of Kings Crossing and 5 miles east of Edinburg on Rt. 675; Shale bank.; 4/25/1983;

M. Daniel 102 (JMUH)

- W. of King's Crossings area. Along Rt 6755 mi. east of Edinburg; along Flood Plains of Little Passage Creek.; 4/25/1983; Parker 3 (JMUH)

- 3 miles north of Kings Crossing on 678.; Roadside.; 4/25/1983; T. Rexroad 1219 (JMUH)

- 5.0 miles east of Edinburg on Rt. 675 on rocky banks along roadside; Sunny, shale barrens.; 4/25/1983; B. Kelley 24 (JMUH)

- State rte. 758, west facing slope, shale barren along road-side.; 4/12/1980; W.A. Wood 21 (JMUH)

- Powells Fort Camp, George Washington national Forest, 2.6 miles north of the intersection of Rt 273 and Rt 66 on Rt 66; Broadleaf, deciduous forest.; Elev. 1200 ft.; 4/22/1978; L. Frey (LFCC)

- Rt \#691, Bear Oak Prescribed Burn, near Va. - W. Va. state line; along roadbank of burned side; Elev. 3005 feet.; 5/16/1981; S. Collins (LFCC)

Viola pubescens Aiton (downy yellow violet)

- $\quad$ Little Sluice Mountain Trail; flower yellow.; 5/6/1980; R.C. Simpson (LFCC)

- 2 mi. NE of Strasburg on Rt \#55 on flood plain on N side of river.; flood plain; flowers: yellow.; 5/1/1975; K.D. Ramsey (LFCC)

Viola sagittata Ait. (arrowleaf violet)

- Devil's Backbone State Forest on the edge of dirt road in forest. Clay soil.; 5/10/2011; 39.04685, -78.42977; Domangue, B. Burkholder 173

Viola sororia Willd. (common blue violet)

- Clearing in wooded area near small cabin.; 5/11/2011; 38.94997,-78.52692; Domangue, C.K. McMullen 196

- Devil's Backbone State Forest on the edge of dirt road in forest. Clay soil.; 5/10/2011; 39.04658, -78.42893; Domangue, B. Burkholder 172

- Meems Bottom Covered Bridge near N. Fork Shenandoah River, 0.5 miles west on Wissler Road.; 4/18/2011; 38.72092,-78.65472; Domangue, J. Courtwright 89

- Mt. Jackson; shale mountain.; 5/1983; J. Kauffman (HAVI)

- 3.4 miles from Detrick, VA. on Rte. 678; moist woods.; 4/14/1982; C. Yost 20 (JMUH)

- On North bank of Stoney Creek about 150 - 200 yards on the South side of Stoney Creek Rd., 1 1.5 miles East of the Rt. 42 junction (Larkin's Grocery), approximately 400 yards downstream from swinging bridge.; 4/13/2001; I. Poplar-Jeffers 5 (JMUH)

- W. of King's Crossings Area. 5 miles east of Edinburg. Along Rt. 675; growing along Flood Plain of Little Passage Creek.; 4/25/1983; Parker 2 (JMUH)

- Mt. Jackson, in the yard at 5875 Gospel St.; 4/4/1990; J.E. Irre 20 (JMUH)

- $\quad$ Power line 300 yds E. of Rt 717; 4 mi NE of Basye; dry, open areas.; 5/9/1984; S. Arey 4 (JMUH)

- $\quad$ Posey Hollow Rd - Rt \#744; south facing slope.; 4/15/1975; S. Miller, S. Henderson, J. Ott (LFCC)

- $\quad$ Maurertown; Rt 6231 mile South Zepp Inter; Elev. 1100 ft.; 4/29/1980; S. Gwyn (LFCC) 
Viola striata Ait. (striped cream violet)

- $\quad$ Elizabeth Furnace Recreation Area a few meters from Passage Creek.; 5/25/2011; 38.92951, -78.32896; Domangue 259

- Edinburg, at the end of Shenandoah Avenue. Growing in a vacant grassy lot.; 4/18/2011; 38.82873,-78.56366; Domangue, J. Courtwright 107

- $\quad 3.0$ miles NE of Lebanon Church on Rt. 628.; 5/21/1988; Chandler 5 (JMUH)

- $\quad 3.0$ mi. NE of Lebanon Church on Route 628. Floodplain of stream.; Edible Parts: Young tender leaves and flowers can be eaten raw, added to soups and stews, or dried and steeped as a tea.; 5/21/1988; Cason, Gordon 13 (JMUH)

- $\quad$ Power line 300 yds. E of Rt 717, 4 m. NE of Basye; creekside.; 5/21/1984; S. Arey 31 (JMUH)

- $\quad$ Elz. Furnace Campground; Flower white.; 4/25/1980; S. Gwyn (LFCC)

- $\quad$ Fort Valley on Rt 678 at Seven Fountains; roadside.; 4/17/1977; R.C. Simpson, J. Bursey (LFCC)

$\diamond$ Viola tricolor L. (johnny jumpup) *

Viscaceae

Phoradendron leucarpum (Raf.) Reveal \& M.C. Johnst. (oak mistletoe)

- Jim Hepner's property off Rt \#623; 100 yds north of the intersection of Rt \#623 and \#604.; 4/1/1980; S.M. Collins, J.A. Hepner (LFCC)

- 1 mile east of Mt. Jackson on short mountain, 1/2 mile west of power line, 3/4 mile North of Fleming Park; Elev. 1500'.; 12/7/1986; D. Hottinger (LFCC)

Vitaceae

Parthenocissus quinquefolia (L.) Planch. (Virginia creeper)

- In weedy lot at the intersection of rt. 11 and rt. 620. Viny plant growing on cedar tree.; 9/1/2011; 38.69479,-78.64812; Domangue 588

- Woodstock Tower; George Washington Nat. Forest.; along roadside.; 10/14/1979; J. Evans (LFCC)

- Woodstock Tower path.; 8/4/1976; C.R. Hupp (LFCC)

Vitis aestivalis Michx. (summer grape)

- $\quad$ FR 274 west.; 7/22/1981; D.E. Miller 19 (JMUH)

- Woodstock; George Washington Nat. Forest; Woodstock Tower.; 10/3/1979; K. Motiska, K. Halstead (LFCC)

- $\quad 31 / 2$ miles west of Edinburg on Rt. 675. South on $6981 / 2$ mile on stream bank on property of Richard Wolverton; Limestone outcroppings stream bank; Elev. 894 ft.; 10/9/1984; A.D. Wolverton, T. Miller (LFCC)

$\diamond$ Vitis labrusca L. (fox grape)

Vitis vulpina L. (frost grape)

- Woodstock, Riverview Park beside Effinger Trail. Vine.; 5/30/2011; 38.87129,-78.49004; Domangue 289 


\section{References}

Brown, H. 2000. Wildland Burning by American Indians in Virginia. Fire Management Today 60(3): 29-39.

Crater PDC (Crater Planning District Commission). 2010. VA PDC Boundaries. Crater PDC, Petersburg, VA. http://gisdata.virginia.gov/downloads/VA_PDC_ boundaries.zip

Gleason, H.A. and A. Cronquist. 1991. Manual of Vascular Plants of Northeastern United States and Adjacent Canada, $2^{\text {nd }}$ edition. New York Botanical Garden, 910 pp.

Dicken, C.L., S.W. Nicholson, J.D. Horton, S.A. Kinney, G. Gunther, M.P. Foose, J. Mueller. 2005. Integrated Geologic Map Databases for the United States: Delaware, Maryland, New York, Pennsylvania, and Virginia, version 1.0. U.S. Geological Survey, Reston, VA. http://pubs.usgs.gov/of/2005/1325/

Harvill, A.M. Jr., T.R. Bradley, C.E. Stevens, T.F. Wieboldt, D.M.E. Ware, D.W. Ogle, G.W. Ramsey and G.P. Fleming. 1992. Atlas of the Virginia Flora. $3^{\text {rd }}$ edition. Burkeville.

Hoffmann, N. and J.V. Horne. 2004. America's Curious Botanist: A Tercentennial Reappraisal of John Bartram 1699-1777. American Philosophical Society, Philadelphia. 227 pp.

Lundholm, J.T. 2009. Plant species diversity and environmental heterogeneity: spatial scale and competing hypotheses. Journal of Vegetation Science 20: 377-391.

McMullen, C.K. 1980. Survey of the vascular flora of Rockingham County, VA. M.S. James Madison University, Harrisonburg.

Miller, R.I. 1986. Predicting rare plant distribution patterns in the southern Appalachians of the south-eastern U.S.A. Journal of Biogeography 13: 293-311.

Newcomb, L. 1977. Newcomb's Wildflower Guide. Little, Brown and Company, Boston, $490 \mathrm{pp}$.

Pausus, J.G. and M.P. Austin. 2001. Patterns of plant species richness in relation to different environments: An appraisal. Journal of Vegetation Science 12: 153-166.

Peters, M.D., C.K. McMullen, and L.M. Hill. 2005. Noteworthy Additions to the Vascular Flora of Rockingham County, VA. Castanea 70(1): 63-69.

Platt, R.B. 1951. An Ecological Study of the Mid-Appalachian Shale Barrens and of the Plants Endemic to Them. Ecological Monographs, 21(4): 269-300. 
Purvis, T.L. 1999. Colonial America to 1763. Facts on File, New York, NY, p. 268.

Rhoads, A.F. and T.A. Block. 2000. The Plants of Pennsylvania: An Illustrated Manual. University of Pennsylvania Press, Philadelphia, PA, 1061 pp.

Rubel, F. and M. Kottek. 2010. Observed and projected climate shifts 1901-2100 depicted by world maps of the Köppen-Geiger climate classification. Meteorologische Zeitschrift 19(2): 135-141.

Shenandoah County Citizens' Advisory Committee. 2005. Comprehensive Plan 2025. http://www.shenandoahcountyva.us/reportscode/comprehensiveplan/

Soil Survey Staff. 1999. Soil Taxonomy: A Basic System of Soil Classification for Making and Interpreting Soil Surveys, $2^{\text {nd }}$ edition. USDA, Natural Resources Conservation Service, $871 \mathrm{pp}$.

Strausbaugh, P.H. and E. Core. 1978. Flora of West Virginia, $2^{\text {nd }}$ edition. Seneca Books, Inc., Morgantown, WV, $1119 \mathrm{pp}$.

The PRISM Climate Group. 2006a. United States Average Monthly or Annual Maximum Temperature, 1971-2000. The PRISM Climate Group, Corvallis, OR. http://prism.oregonstate.edu/index.phtml

The PRISM Climate Group. 2006b. United States Average Monthly or Annual Minimum Temperature, 1971 - 2000. The PRISM Climate Group, Corvallis, OR. http://prism.oregonstate.edu/index.phtml

USDA, National Agricultural Statistics Service. 2009. 2007 Census of Agriculture. Virginia State and County Data. http://www.agcensus.usda.gov/Publications/ 2007/Full_Report/Volume_1,_Chapter_2_County_Level/Virginia/vav1.pdf

USDA, Natural Resources Conservation Service. 2008. Soil Survey Geographic (SSURGO) database for Shenandoah County, Virginia. USDA, NRCS, Fort Worth, TX. http://soildatamart.nrcs.usda.gov/

USDA, Natural Resources Conservation Service. 2012. The PLANTS Database. National Plant Data Team, Greensboro, NC. http://plants.usda.gov

USDA, Soil Conservation Service. 1991. Soil Survey of Shenandoah County, Virginia. pp. 1-3.

USGS (U.S. Geological Survey). 2009. 1-Arc Second National Elevation Dataset. USGS, Sioux Falls, SD. http://seamless.usgs.gov/

USGS (U.S. Geological Survey). 2011. NLCD 2006 Land Cover. USGS, Sioux Falls, SD. http://seamless.usgs.gov/ 
USDC (U.S. Department of Commerce), National Climatic Data Center. 2010. Summary of Monthly Normals: $1981-2010$. NCDC, Asheville, NC.

http://www1.ncdc.noaa.gov/pub/orders/42012.pdf

USDC (U.S. Department of Commerce), U.S. Census Bureau, Geography Division. 2010a. TIGER/Line Shapefile, 2010, county, Shenandoah County, VA, Linear Hydrography County-based Shapefile. http://www.census.gov/geo/www/tiger

USDC (U.S. Department of Commerce), U.S. Census Bureau, Geography Division. 2010b. TIGER/Line Shapefile, 2010, county, Shenandoah County, VA, Current Area Hydrography Shapefile. http://www.census.gov/geo/www/tiger

VA-DCR (Virginia Department of Conservation \& Recreation). 2012a. DOF Managed Lands. http://www.dcr.virginia.gov/natural_heritage/documents/dof.zip

VA-DCR (Virginia Department of Conservation \& Recreation). 2012b. USFS Managed Lands. http://www.dcr.virginia.gov/natural_heritage/documents/usfs.zip

Virginia Botanical Associates. 2012. Digital Atlas of the Virginia Flora. Virginia Botanical Associates, Blacksburg. http://www.vaplantatlas.org/

Wayland, J.W. 1980. A History of Shenandoah County, Virginia, $2^{\text {nd }}$ edition. Regional Publishing Company, Baltimore, pp. 33-39, 47-58, 99-103.

WRAC (Shenandoah County Water Resources Advisory Committee). n.d. Water in Shenandoah County: A Primer. http://www.shenandoahconnection.com/ waterprimer.pdf

Young, J., G. Fleming, W. Cass, and C. Lea. 2009. Vegetation of Shenandoah National Park in Relation to Environmental Gradients, Version 2.0. National Park Service, Philadelphia, PA. 